\title{
CURRENT-PUMPING PERFORMANCE OF AXIAL-SYMMETRIC SUPERCONDUCTING DYNAMO DESIGNS
}

\author{
BY \\ SIN HOI PHANG
}

\begin{abstract}
A thesis
submitted to the Victoria University of Wellington

in fulfilment of the requirements for the degree of

Master of Science

in Physics
\end{abstract}

Victoria University of Wellington

2017 


\section{Abstract}

In this project, the current pumping performances of three high temperature superconducting (HTS) dynamos (mechanically-rotating flux pumps) were investigated. These flux pumps (FPs) were identified as the Gen 1, Gen 2 and Gen 4 FP respectively. They were modelled using simple DC equivalent circuits and their performances were defined by three output parameters: DC open-circuit voltage $\left(V_{o c}\right)$, dynamic resistance $\left(R_{d}\right)$ and DC short-circuit current $\left(I_{s c}\right)$. The experimental results showed that these FPs produced non-zero DC output voltages across their stators and each supplied DC output currents into series connected superconducting circuits.

The Gen 1 FP was cooled with liquid nitrogen and operated at $77 \mathrm{~K}$. The stators were made from 12-mm wide copper-coated yttrium barium copper oxide (YBCO) HTS conductors. The objective of the experiment was to investigate the effect of the rotor magnet size, geometry and orientation (with respect to the stators) on the FP performance. The design parameters, based on the width and the length of the magnet with respect to the stator, for optimal FP performance were determined.

The Gen 2 FP was also cooled with liquid nitrogen and operated at $77 \mathrm{~K}$. The stators were made from 46-mm wide Ag-coated YBCO HTS conductors. The objective of the experiment was to investigate the effect of stator width on the FP performance at various magnet frequencies. This was done by slitting two of the stators to form parallel stators with equal width, but smaller than the original conductor width. The experimental results showed that the FP performance was highly dependent on the operating frequency. Three operating frequency regimes were observed: low, mid and high. At high frequency, the $V_{o c}$ decreased and the $R_{d}$ increased significantly. This was attributed to the local heating effect in the stator due to the increase in the current density. As a result, the FP produced zero output current in this regime. The AC voltage waveforms measured across the stators in different frequency regimes were very different in shapes and amplitudes. Therefore, these waveforms can be used to identify the operating frequency regime of the FP. At some operating frequencies in the mid frequency regime, bi-stable operating modes were observed, each mode resulted in different output parameters.

The Gen $4 \mathrm{FP}$ was constructed in this project. It was cooled via thermal conduction method using a cryorefrigerator system. It had an axial-symmetric stator design where the shape of the stator is cylindrical. The width of the stator was considerably larger compared with the Gen 1 and Gen 2 FPs. The experiment objective was to investigate whether this 
design would produce a DC current, and then characterise the FP performance at different operating temperatures. The experimental results showed that the time-averaged DC output voltage of the Gen 4 FP was much higher compared with the Gen 1 and Gen 2 FPs. The reason is that it produced a DC output voltage for almost the entire rotor cycle. The experimental results also indicated that the Gen 4 FP has the capability to produce output currents in the $\mathrm{kA}$ range. 


\section{Acknowledgement}

I would like to thank my supervisors Dr Chris Bumby and Dr Rod Badcock. Without their support and guidance throughout the project this thesis would not have been possible.

I would also like to thank Olly (A. E.) Pantoja for his technical support, discussions on the project, and proof-reading my thesis draft. Throughout the project, he helped me with the setup of the experiment systems and data measurement. I am grateful to Dr Zhenan Jiang for providing me invaluable advice and information. I am also grateful to Dr Evgeny Talantsev in helping me with the current coil sensor calibration and flux pump stator preparation.

I would like to thank Gennady Sidorov for helping me with the vacuum system and providing electrical engineering support. I also would like to thank Mike Davies and Kent Hamilton for their help in setting up the gantry and their support in electrical and mechanical engineering.

I am grateful to Dr Guy Dubuis, Dr Shen Vun Chong, Benjamin Parkinson, Dr Nick Strickland and Dr Mike Staines. Throughout the project, I encountered numerous technical challenges, which were solved with their help and advice. I am also grateful to Jeannie Redman and Ursula Muavae for their support in administration matters and the purchase of items for the projects. I also would like to thank the New Zealand Ministry of Business, Innovation and Employment, for awarding the scholarship to me for my Masters study.

Last but not least, I would like to express my gratitude to my parents, my wife and my children for their continuing support and patience. 


\section{Contents}

1 Introduction $\quad 1$

2 Literature Review $\quad 3$

2.1 Type II Superconductors . . . . . . . . . . . . . . . . . . . . 3

2.2 High- $T_{C}(\mathrm{HTS})$ Oxides Wire . . . . . . . . . . . . . . . . 4

$2.2 .12 \mathrm{G}$ HTS Wires $\ldots \ldots \ldots \ldots \ldots$

2.2.2 Power Law Relationship . . . . . . . . . . . . . . . 5

2.2.3 Flux Flow Resistivity . . . . . . . . . . . . . . . . . . 6

2.3 Flux Pump . . . . . . . . . . . . . . . . . . . 6

2.3.1 LTS Flux Pump . . . . . . . . . . . . . . 7

2.3.2 HTS Flux Pump ..................... 8

2.3.2.1 The Dynamic Resistance . . . . . . . . . . . . . 8

2.3.2.2 The DC Voltage Generation in a HTS FP . . . . . . . . 8

2.4 DC Behaviour and the I-V Characteristics of the Flux Pump . . . . . . . . . 11

3 Gen 1 and Gen 2 FP Systems Experimental Designs 14

3.1 RRI First Generation Flux Pump (Gen 1) . . . . . . . . . . . . . . . . . . 14

3.1.1 HTS Stator and Mechanical Construction . . . . . . . . . . . . . 14

3.1.2 Schematic Diagram and Signal Acquisition . . . . . . . . . . . . 16

3.2 RRI Second Generation Flux Pump (Gen 2) . . . . . . . . . . . . . . . 17

3.2.1 HTS Stator and Mechanical Construction . . . . . . . . . . . . 17

3.2.2 Schematic Diagram and Signal Acquisition . . . . . . . . . . . . . . 19

4 Gen 1 and Gen 2 FP Systems Experimental Results 21

4.1 Gen 1 Flux Pump System Experimental Results . . . . . . . . . . . . . . . . 21

4.1.1 Experiment Samples Setup . . . . . . . . . . . . . 21

4.1.2 The AC Waveforms and the Open-Circuit Voltage Generation . . . . 22

4.1.3 FP Performance (6 mm magnet, vertical orientation) . . . . . . . . . 24

4.1 .4 Vertical Orientation . . . . . . . . . . . . . . . . . . 27

4.1.4.1 Open-Circuit Voltage . . . . . . . . . . . . . . . . 29

4.1.4.2 Dynamic Resistance . . . . . . . . . . . . . . . . 32

4.1.4.3 Short-Circuit Current . . . . . . . . . . . . . 32

4.1.5 Angled Orientation . . . . . . . . . . . . . . . . . . . 32 
4.1.5.1 Open-Circuit Voltage . . . . . . . . . . . . . . 34

4.1.5.2 Dynamic Resistance . . . . . . . . . . . . . . . . . 34

4.1.5.3 Short-Circuit Current . . . . . . . . . . . . . . 34

4.1 .6 Horizontal Orientation . . . . . . . . . . . . . . . . . . 35

4.1.6.1 Open-Circuit Voltage . . . . . . . . . . . . . . . . 37

4.1.6.2 Dynamic Resistance . . . . . . . . . . . . . . . . . . 37

4.1.6.3 Short-Circuit Current . . . . . . . . . . . . . . . . . 37

4.1.7 Fixed Magnet Size (3 $\mathrm{mm} \& 6 \mathrm{~mm}$ Magnets $)$. . . . . . . . . . . . . 37

4.1 .8 Fixed Magnet Size $(12 \mathrm{~mm}$ Magnet $)$. . . . . . . . . . . . . . . . . 40

4.1 .9 Summary . . . . . . . . . . . . . . . . . . . 42

4.2 Gen 2 Flux Pump System Experimental Results . . . . . . . . . . . . . . . . 43

4.2.1 Experiment Samples Preparation . . . . . . . . . . . . . . . 43

4.2.2 The AC Waveforms and the Open-Circuit Voltage Generation . . . . 44

4.2 .3 Contact Resistance . . . . . . . . . . . . . . . . . . . 45

4.2.4 The Performance and the Frequency Dependent Behaviour of the FP 46

4.2.5 The AC Waveforms and the Operating Frequency Regimes . . . . . . 51

4.2 .6 Bi-stable Mode . . . . . . . . . . . . . . . . . . 55

4.2 .7 Summary . . . . . . . . . . . . . . . . . . 56

5 Gen 4 FP System Experimental Design and Commission 58

5.1 RRI Fourth Generation Flux Pump (Gen 4 System) . . . . . . . . . . . . . . 58

5.1 .1 The Gen 4 Cryogenic System . . . . . . . . . . . . . 58

5.1 .2 The HTS FP Construction . . . . . . . . . . . . . . . . 60

5.1.2.1 HTS Stator Assembly . . . . . . . . . . . . . 61

5.1 .2 .2 HTS FP Circuit . . . . . . . . . . . . . 62

5.1.2.3 The Construction and Installation of the Stator and the Leads 63

5.1.2.4 Temperature Monitor and Control of the Stator . . . . . . . 66

5.1.3 HTS Current Coil Assembly and Hall Sensor Calibration . . . . . . . 68

5.1.4 Schematic Diagram and Signal Acquisition . . . . . . . . . . . . 70

5.2 Cooling Experiments . . . . . . . . . . . . . . . . . . . . . 72

5.2.1 Initial Cooling Tests and PT100 Sensors Calibration . . . . . . . . . . 73

5.2.2 Removal of the Thermal Break . . . . . . . . . . . . . . 75

5.2 .3 Thermal Radiation Shield . . . . . . . . . . . . . . . . . . 77

5.2 .4 Thermal Bus . . . . . . . . . . . . . . . . . . . 78

6 Gen 4 FP System Experimental Results $\quad 81$

6.1 Temperature Monitoring . . . . . . . . . . . . . . . . 82

6.2 Temperature Stability _. . . . . . . . . . . . . . . . . . . 82

6.3 The AC Waveforms and the Open-Circuit Voltage Generation . . . . . . . . 83

6.4 The DC Equivalent Circuit, Contact Resistances and Flux Pump Voltage . . 86

6.5 The FP Performance . . . . . . . . . . . . . . . . . . . . 87 
6.5.1 Current vs Time Plot . . . . . . . . . . . . . . . . . 88

6.5.2 $I-V$ Curves . . . . . . . . . . . . . . . . . . . . 88

6.5.3 Line fit error . . . . . . . . . . . . . . . . . . . 91

6.5.4 FP Performance Parameters . . . . . . . . . . . . . . . . . . 92

6.5.5 Frequency Normalised Plots . . . . . . . . . . . . . . . . 96

6.6 Summary . . . . . . . . . . . . . . . . . . . . . . . 97

7 Conclusions $\quad 99$

7.1 Gen 1 System . . . . . . . . . . . . . . . . . . . . . . . . . . 99

7.2 Gen 2 System . . . . . . . . . . . . . . . . . . . 100

7.3 Gen 4 System . . . . . . . . . . . . . . . . . . . 100

7.4 Applications . . . . . . . . . . . . . . . . . . 101

A 50-watt Constant-current Heater Power Supply 105

A.1 Heater Circuit Design . . . . . . . . . . . . . . . . . . . . 105

A.1.1 Voltage-controlled Precision Current Sink . . . . . . . . . . . . . 105

A.1.2 The Power Transistor . . . . . . . . . . . . . . . . . . . . 106

A.1.3 NI-9263 Maximum Control Voltage and Sensing Resistor Value . . . . 106

A.1.4 Other Considerations . . . . . . . . . . . . . . . . 107

A.2 Heatsink and Transistor Junction Temperature . . . . . . . . . . . . . . . . 107

A.3 Prototype Test and Final Design _. . . . . . . . . . . . . . . . 109

B $10-\mu$ A Constant Current Source for DT-670 Diode Sensors 111 


\section{List of Figures}

2.1 Type II superconductor. . . . . . . . . . . . . . . . . . . . 3

2.2 2G HTS tape . . . . . . . . . . . . . . . . . . . . 4

2.3 HTS tape power law. . . . . . . . . . . . . . . . 5

2.4 HTS FP. . . . . . . . . . . . . . . . . . . . 7

2.5 LTS drum FP. . . . . . . . . . . . . . . . . . . . . 7

2.6 A simple LTS FP. . . . . . . . . . . . . . . . . . . . 8

2.7 HTS FP AC waveforms. . . . . . . . . . . . . . . . . . . . . . 9

2.8 HTS tape and magnet. . . . . . . . . . . . . . . . . . 10

2.9 HTS FP AC model. . . . . . . . . . . . . . . . . . . . . . . . . . 10

2.10 HTS FP DC model. . . . . . . . . . . . . . . . . . . . . . . . 11

2.11 HTS FP $I-V$ curve. . . . . . . . . . . . . . . . . . . . . . . . . . 12

2.12 HTS FP current vs time plot. . . . . . . . . . . . . . . . 13

3.1 Gen 1 HTS FP. . . . . . . . . . . . . . . . . . . . . . . . 14

3.2 FP $I-V$ curves measured with coils and current supply. . . . . . . . . . . 15

3.3 Gen 1 FP system schematic and signal acquisition. . . . . . . . . . . . . 16

3.4 Gen 2 FP arrangement. . . . . . . . . . . . . . . . . . . . . 18

3.5 Gen 2 FP system schematic and signal acquisition. . . . . . . . . . . . . . 19

4.1 Vertically orientated magnet AC waveforms. . . . . . . . . . . . . . . 23

$4.2 \quad$ FP $I-V$ curves. . . . . . . . . . . . . . . . . . . . . . . . 24

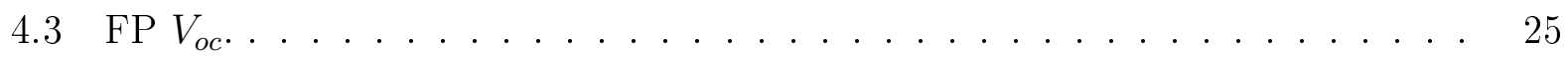

4.4 FP performance parameters -6 mm magnet. . . . . . . . . . . . . 26

4.5 FP performance parameters - vertical magnets. . . . . . . . . . . . . 28

4.6 Opera simulations for vertical magnets. . . . . . . . . . . . . . . . . . . . . 29

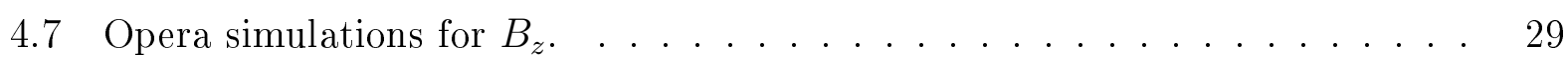

$4.8 \Delta V / f$ waveforms $-6 \mathrm{~mm}$ vs $12 \mathrm{~mm}$ magnets. . . . . . . . . . . . . . . . 31

4.9 FP performance parameters for angled orientation. . . . . . . . . . . . . . 33

4.10 Opera simulations for angled magnets. . . . . . . . . . . . . . . . 34

4.11 FP performance parameters for horizontal orientation. . . . . . . . . . . 36

4.12 Opera simulations for horizontal magnets. . . . . . . . . . . . . . . . . . 37

$4.133 \mathrm{~mm}$ magnets orientation comparisons. . . . . . . . . . . . . . . . . 38

4.146 mm magnets orientation comparisons. . . . . . . . . . . . . . . . . 39 
$4.1512 \mathrm{~mm}$ magnet orientation comparisons. . . . . . . . . . . . . . . . . . 41

4.16 Slit tapes. . . . . . . . . . . . . . . . . . . . . . . . . . . . 44

4.17 Stators installed on the FP system. . . . . . . . . . . . . . . . . 44

4.18 Stator III $V_{o c} / f$ waveforms. . . . . . . . . . . . . . . . . . . . 45

4.19 Stator III decay current curve fit. . . . . . . . . . . . . . . . . . . . . 46

4.20 Stator III $I-V$ curve. . . . . . . . . . . . . . . . . . . . . . . . . . . . . 47

4.21 Stator III DC current vs time plot. . . . . . . . . . . . . . . . . . 48

4.22 Stator I $I-V$ curve. . . . . . . . . . . . . . . . . . . . . . . . . . . . . . 48

4.23 Stator II $I-V$ curve. $\ldots \ldots \ldots \ldots$

4.24 Gen 2 FP performance. . . . . . . . . . . . . . . . . . . . . 50

4.25 Stator I $V_{o c} / f$ waveforms. . . . . . . . . . . . . . . . . . . . . . 52

4.26 Stator III $V_{o c} / f$ waveforms. . . . . . . . . . . . . . . . . . . . 53

4.27 Stator III $\Delta V / f$ at $27 \mathrm{~Hz} \ldots \ldots \ldots \ldots \ldots \ldots$

4.28 Stator III bi-stable mode at $111 \mathrm{~Hz} \ldots \ldots$. . . . . . . . . . . . 55

4.29 Stator III time plot at $111 \mathrm{~Hz} \ldots \ldots \ldots$. . . . . . . . . . . . . 56

5.1 Gen 4 vacuum chamber. RRI Solidworks model. . . . . . . . . . . . . . . . . 59

5.2 AL63 cooling capacity. . . . . . . . . . . . . . . . . . . 59

5.3 Gen 4 test rig full assembly. . . . . . . . . . . . . . . . . . . . . . 60

5.4 Stator assembly. . . . . . . . . . . . . . . . . . . . 62

5.5 Gen 4 HTS FP circuit. . . . . . . . . . . . . . . . . . 63

5.6 Mounting of the HTS stator onto the sapphire tube. . . . . . . . . . . . . 64

5.7 HTS flyleads soldered onto the copper support pieces and stator. . . . . . . . 64

5.8 HTS current lead soldering. . . . . . . . . . . . . . . . . 65

5.9 Current lead and flylead connections. . . . . . . . . . . . . . 65

5.10 Soldering heater and the power supply. . . . . . . . . . . . . . . . 66

5.11 Stator temperature control and monitor. . . . . . . . . . . . . . . . . 67

5.12 Gen 4 temperature sensors. . . . . . . . . . . . . . . . . . . . . . 67

5.13 Gen 4 current coil assembly. . . . . . . . . . . . . . . . . 68

5.14 Gen 4 system HTS current coil Hall sensor calibration. . . . . . . . . . . . . 69

5.15 Gen 4 HTS coil Hall sensor calibration curve. . . . . . . . . . . . . . . 70

5.16 Gen 4 FP schematic. . . . . . . . . . . . . . . . . . . . . . . . 71

5.17 G4 FP system voltage taps. . . . . . . . . . . . . . . . . . 72

5.18 PT100 and DT670 mounting. . . . . . . . . . . . . . . . . . 73

5.19 Initial cooling test. . . . . . . . . . . . . . . . . . . . . . 74

5.20 Sensor calibration curves. . . . . . . . . . . . . . . . . . . . 75

5.21 Cooling test - thermal break removed. . . . . . . . . . . . . . . . . . 76

5.22 MLI . . . . . . . . . . . . . . . . . . . . . . 77

5.23 Cooling test with MLI installed. . . . . . . . . . . . . . . 78

5.24 Thermal bus installation. . . . . . . . . . . . . . . . . . . . . 79

5.25 Gen 4 system experiment final setup. . . . . . . . . . . . . . . . . . . . 79 
5.26 Cooling test - thermal bus installed. . . . . . . . . . . . . . . 80

6.1 Gen 4 temperature sensors locations. . . . . . . . . . . . . . . . . 82

6.2 Gen 4 temperature stability. . . . . . . . . . . . . . . . . . . . . 83

6.3 Gen $4 V_{o c} / f \mathrm{AC}$ waveforms. . . . . . . . . . . . . . . . . . . . . . 85

6.4 Gen 4 FP DC equivalent circuit. . . . . . . . . . . . . . . . . . . 86

6.5 Gen 4 FP current vs time. . . . . . . . . . . . . . . . . . . 88

6.6 Gen $4 I-V$ at $50 \mathrm{~K} . \ldots \ldots \ldots \ldots$

6.7 Gen $4 I-V$ at $60,70 \& 80 \mathrm{~K} . \ldots \ldots \ldots$

6.8 FP performance vs frequency. . . . . . . . . . . . . . . . . . . 93

6.9 FP performance vs temperature. . . . . . . . . . . . . . . . . . . . . . 95

6.10 Gen 4 FP frequency normalised plots. . . . . . . . . . . . . . . . . . 97

A.1 $50 \mathrm{~W}$ power supply. . . . . . . . . . . . . . . . . . . . . 105

A.2 Power transistor power dissipation. . . . . . . . . . . . . . . . . 107

A.3 Thermal modelling. . . . . . . . . . . . . . . . . . . . . . . . . . . . 108

A.4 Power supply prototype test. . . . . . . . . . . . . . . . . . 109

A.5 Heater power supply test result. . . . . . . . . . . . . . . . . . . . . . 109

A.6 $50 \mathrm{~W}$ power supply final design. . . . . . . . . . . . . . . . . . . . . 110

B.1 DT-670 current source. . . . . . . . . . . . . . . . 111 


\section{Chapter 1}

\section{Introduction}

A High temperature superconducting (HTS) dynamo is a device that is capable of supplying direct current into HTS circuits. A typical application is to energise HTS coils for high magnetic field generation. The current can be supplied to the coils from an external power supply via current leads. However, this approach introduces heat into the cryogenic system via the wire connections [40]. With a HTS dynamo the physical connections between the power supply and coil are not required. It has been shown that this approach reduces the heat load "by a factor of 4.5 when compared to using a conventional" power supply [40]. An alternative term for dynamo is mechanically rotating flux pump (FP), and these terms are used interchangeably in this thesis.

The mechanical HTS FPs used in this project were designed and constructed in Robinson Research Institute (RRI). These FPs consist of a magnet rotor and a HTS stator. High temperature superconducting wires were used to make the stator because of their superior performance in terms of critical temperature and critical magnetic field strength compared with low temperature superconductor. This will be discussed in chapter 2. The rotor holds permanent magnets and as it rotates, the magnets pass over the HTS stator. This generates a DC voltage across the stator, and if a load (e.g. a HTS current coil) is connected across it, a current is injected into the load. This process is repeated and the current in the circuit accumulates over time.

A topic that appears frequently is the generation of DC voltage by a HTS FP via magnetic induction. This is possible because as the magnet passes over the stator, the transport current interacts with the external AC magnetic field. This process causes a resistivity change in the material and produces the non-zero output voltage across the stator [5]. This resistivity in the superconducting occurs due to the interaction between the flux and the current [13, 19]. In an ideal situation the maximum output current, known as the short-circuit current, that can be supplied by a HTS FP is only limited by the internal resistance of the stator. This internal resistance is known as the dynamic resistance and it arises due to the flux flow in the superconducting material caused by the interaction between the transport current and the AC field. When the FP is in an open circuit condition $(I=0 \mathrm{~A})$, the DC voltage measured across the stator equals the open-circuit voltage. The three parameters: open- 
circuit voltage; dynamic resistance and short-circuit current characterise the performance of a FP. These topics will also be discussed in chapter 2 .

The experiments conducted in this project were performed using three different types of mechanical HTS FP and experimental systems. For the first type, known as the Gen 1 system, rotor magnets of different sizes and orientations were used to investigate the impact of the magnet geometry on the performance of the FP. The whole system was submerged in a liquid nitrogen bath and cooled to $77 \mathrm{~K}$. From the experimental results, the magnet geometry and stator width for an optimal FP performance were determined. The results have been published in [3].

In the second experimental setup, a mechanical HTS FP known as the Gen 2 system was used. Unlike the first FP, the moving parts were located outside of the cryogenics environment. The advantage of using this approach is that the cryogenic system does not suffer heat loss associate with the moving parts $[4,6]$. Another feature of this FP is that there are nine permanent magnets spaced equally on the rotor. This increases the operating frequency of the FP by a factor of nine compared with the Gen 1 system. For this reason, the high frequency performance of the HTS FP was investigated. The experimental results showed that the performance of the FP was frequency dependent. At extremely high operating frequency the FP output current diminished dramatically because the stator appeared to be driven to thermally quenched state. The experimental results have been published in [7]. The design of the Gen 1 and Gen 2 systems and the experimental results will be discussed in chapter 3 and chapter 4 respectively.

In the third part of this project, an axial-symmetric mechanical HTS FP, called the Gen 4 system, was installed and used to investigate the current pumping performance at different temperatures $(40-80 \mathrm{~K})$. Unlike the Gen 1 and Gen 2 systems mentioned earlier, this FP was cooled via thermal conduction method, using a cryorefrigerator and a vacuum system. A similar design and cooling method has been used before [40, 15] where a coil temperature of $30 \mathrm{~K}$ and FP temperature range from 70 to $80 \mathrm{~K}$ have been achieved. For this project, the low temperature of the FP was extremely difficult to achieve. This was partly due to the thermal mass of the apparatus and partly due to the thermal resistances in the system. The technical difficulties were eventually solved and coil temperature of about $20 \mathrm{~K}$ and FP temperature of $30 \mathrm{~K}$ were achieved. The design, installation and commission of the Gen 4 system will be discussed in chapter 5 .

The results of the Gen 4 system experiments will be discussed in chapter 6 . It was found that the Gen 4 system was much more effective in terms of voltage output compared with the Gen 1 and Gen 2 systems. This is because the symmetry of the stator enabled the FP to produce a non-zero DC voltage for almost the entire rotor cycle. The results also indicate that the Gen 4 system has the capability to produce output currents in the kA range. 


\section{Chapter 2}

\section{Literature Review}

This thesis is about the development and the experimental results of prototype superconductor flux pumps (FP) employing high temperature superconductors. In this chapter, the type II high temperature superconductors (HTS) are introduced. The mechanism behind the DC voltage generation of a flux pump, the DC model and the parameters that describe the performance are discussed.

\subsection{Type II Superconductors}

There are two types of superconductors: type I and type II. When a Type I superconductor is cooled below the critical temperature $\left(T_{c}\right)$, the resistance and the magnetic field inside the material is zero. As a result, type I superconductors expel weak external magnetic fields. Type II superconductors have three different states, depending on the strength of the external magnetic field $B$. This is shown in figure 2.1. If the external field is at or lower than $B_{c 1}$, the material is in the superconducting state and it expels the external field. If the field is above $B_{c 1}$ but lower than $B_{c 2}$, the material is in a mixed state (superconducting and normal) because some of the flux penetrates into the material. Above $B_{c 2}$, the superconductivity is destroyed and the material becomes a normal conductor [10].

This content is not available. Please refer to the print

version for access.

Figure 2.1: Three different states of a Type II superconductor. Source:[10].

The fact that type II superconductors operate in mixed states means that some can with- 
stand very strong external field [10]. Another important feature of type II superconductors is that they have higher critical current density $J_{c}$. All these features make type II superconductors more useful than type I in practical applications. In this project, type II high temperature superconductors were used in the experiments.

\subsection{High- $T_{C}$ (HTS) Oxides Wire}

Superconductors are also grouped into two categories based on the value of $T_{c}$. The low temperature superconductors (LTS) require cooling to about $4 \mathrm{~K}$ with liquid helium. The other group is the high temperature superconductor (HTS). A superconductor with a critical temperature equal to or greater than $77 \mathrm{~K}$ is considered as a high temperature superconductor.

A group of very important type II HTS superconductors are made from ceramic material. An example is yttrium barium copper oxide (YBCO), which is used in this project. These ceramic-based type II superconductors have high $T_{c}(>90 \mathrm{~K})$ and can withstand external fields $>100 \mathrm{~T}$ parallel to the HTS wire at $4.2 \mathrm{~K}$. The higher $T_{c}$ of HTS is an advantage because for most applications liquid nitrogen $\left(N_{2}\right)$ can be used as the cryogen. Liquid $N_{2}$ has a boiling point of $77 \mathrm{~K}$ and is cheap to obtain and use.

\subsubsection{G HTS Wires}

In this project coated conductors (also known as $2 \mathrm{G}$ ) HTS wires (tapes) were used. They are made from a barium cuprate thin film (e.g. YBCO) deposited upon a buffer layer on a metal substrate (for mechanical support), and coated with a protective metal (e.g. copper) layer. An example of a $2 \mathrm{G}$ tape construction is shown in figure 2.2. $2 \mathrm{G}$ tapes were chosen for this project because they have high critical current $I_{c}$ in perpendicular external field and are commercially available from a few manufacturers. The tapes used in this project were manufactured by the companies called SuperPower Inc. and American Superconductor.

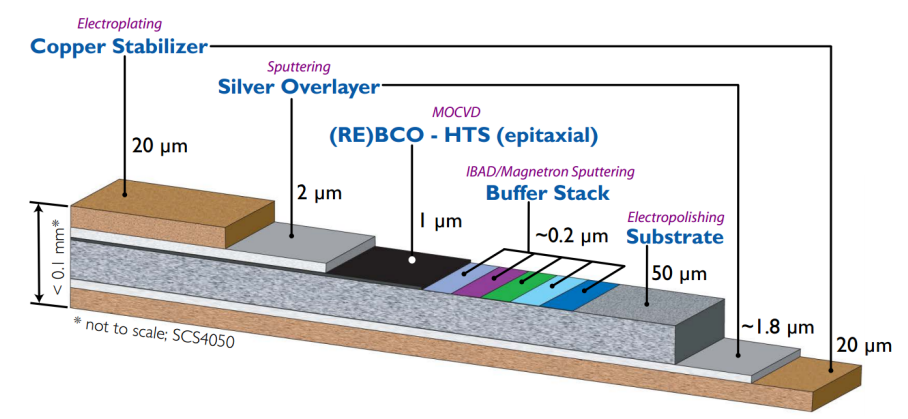

Figure 2.2: 2G HTS tape construction. Source:[35] 


\subsubsection{Power Law Relationship}

Superconductors become normal conductors when the current density in the material is higher than a critical value $J_{c}$. For this reason, it very important to know the value of $J_{c}$ so that the materials are not driven into normal state. However, $J_{c}$ is not very well defined in HTS due to its smooth transition from superconducting to normal states, known as the flux-flow regime. It has been found that the voltage-current $(V-\mathrm{I})$ curve of HTS follows a power law relationship [33] and this allows $J_{c}$ to be determined. The relationship is given by:

$$
E=E_{c}\left(\frac{J}{J_{c}}\right)^{n}
$$

where $J_{c}$ is the critical current density, which corresponds to $E_{c}$. $E_{c}$ is the the critical electric field and is defined as the value when the voltage developed across a section of the tape is equal to $1 \mu \mathrm{V} / \mathrm{cm}$.

The curvature of the V-I curve is quantified by the constant $n$, which typically varies between 20 to 40 for HTS. A higher $n$ value implies a sharper curve. The power law relationship allows the $J_{c}$ of a HTS to be quantified. For example, figure 2.3 shows the $V-I$ curve measured from a SuperPower YBCO tape and the power-law curve was fitted to the data. For this sample the $n$ value is 33 and the critical current is about $162.5 \mathrm{~A}$.

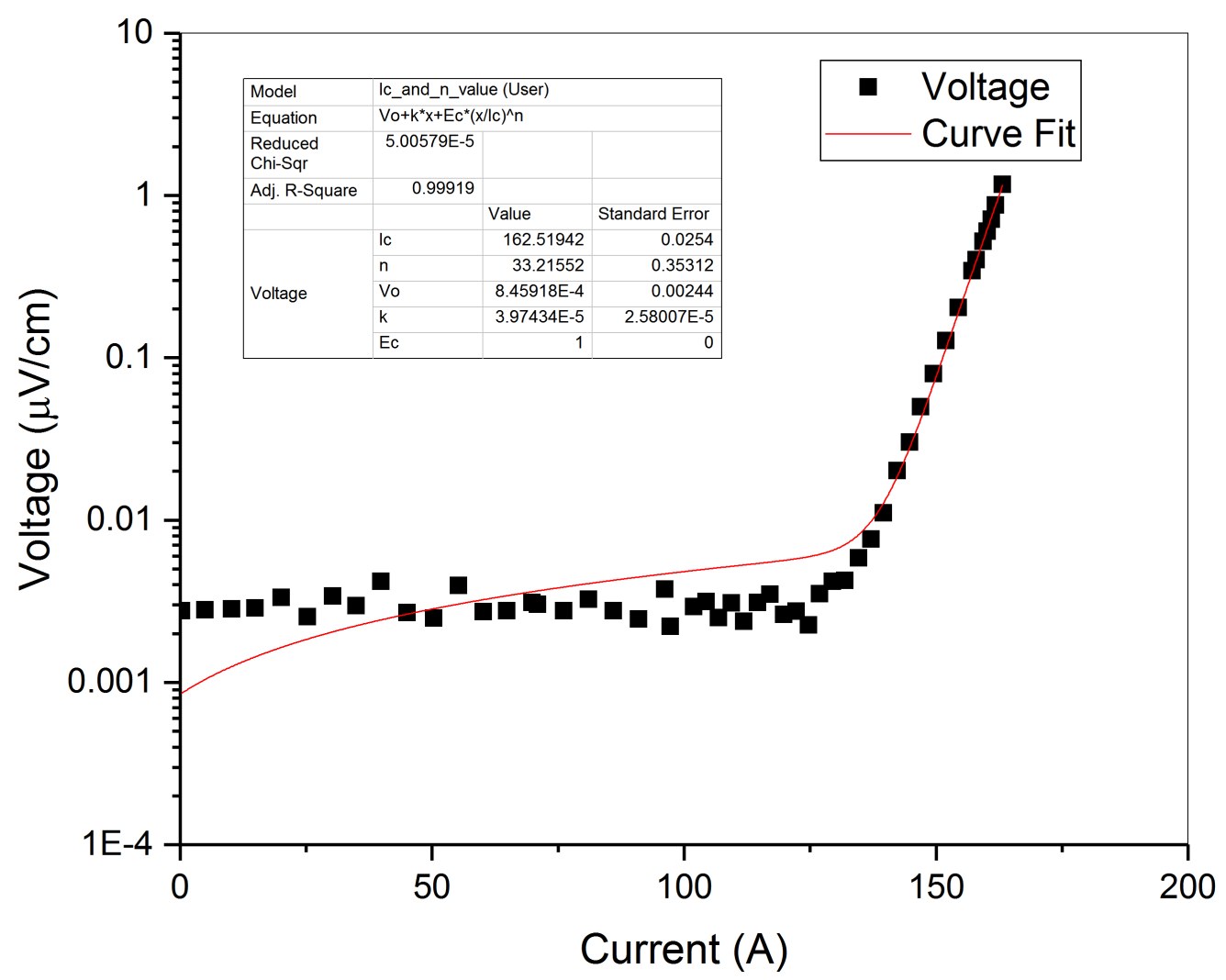

Figure 2.3: HTS tape $V-I$ characteristics and n value obtained from measurements. The calculated $I_{c}$ is 162.5 A. Data source: RRI 


\subsubsection{Flux Flow Resistivity}

As discussed earlier, a strong magnetic field can penetrate into the material of a type II superconductor. This creates quantised flux vortices in the material surrounded by circulating supercurrent [13].

The superconductivity can be destroyed by increasing the DC transport current. When the transport current density reaches $J_{c}$, the material is in the mixed state and the resistance is no longer zero, otherwise voltage cannot developed across the wire. The resistivity in the superconductor is attributed to the flow of the flux in the material. This is caused by the Lorentz force interaction between the flux and the transport current [13].

\section{$2.3 \quad$ Flux Pump}

A flux pump (FP) is a device that is capable of injecting DC currents into a superconducting circuit via magnetic field induction. Therefore, it avoids the heat losses in the cryogenic system introduced by the electrical wires connecting the power source and the load. The applications of HTS FP for the purpose of energising HTS magnets have been investigated in $[40,15]$. It was found that "a reduction in heat load by a factor of 4.5 " was achieved "when compared to using a conventional" power supply [40, 15].

An example of a simple HTS FP is shown in figure 2.4. A similar design has been used in this project (see chapter 3). It consists of a stationary HTS tape and a permanent magnet mounted on a rotating holder. As the magnet passes the HTS tape (stator), a DC opencircuit voltage $\left(V_{o c}\right)$ is developed across the tape. A load (e.g. a superconductor coil) is connected to the stator and forms a complete circuit with the stator so that the DC currents can be injected into the load. Both LTS and HTS have been used in previous works to construct FPs and are discussed separately below. 


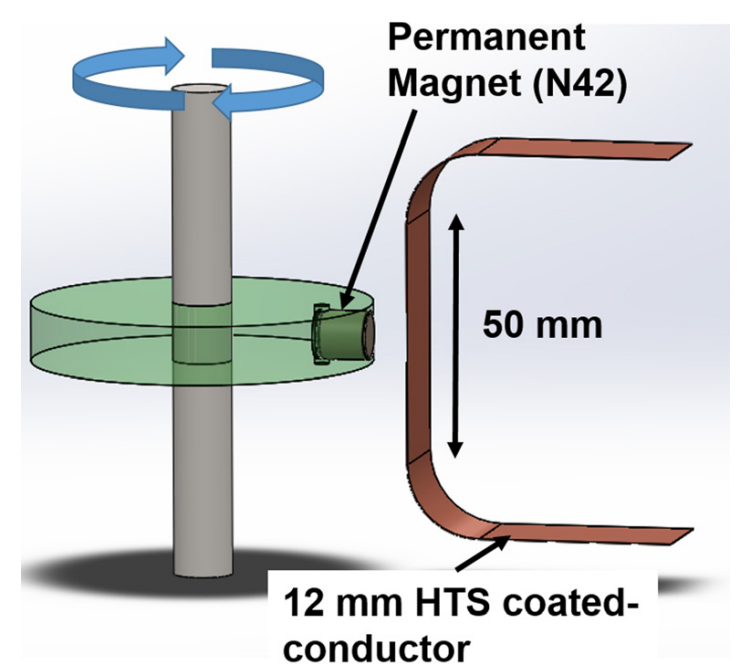

Figure 2.4: The arrangement of a HTS FP consists of a HTS tape and a rotating magnet. Source:[5].

\subsubsection{LTS Flux Pump}

A common LTS FP design is the axial-symmetric (drum) type [24, 39, 23, 2]. An example of the drum FP is shown in figure 2.5. It consists of a thin cylindrical superconducting sheet that forms the stator. The rotor holds the permanent magnets and rotates on the flux pump axis. A complete circuit connection is formed between stator and the load via wires.

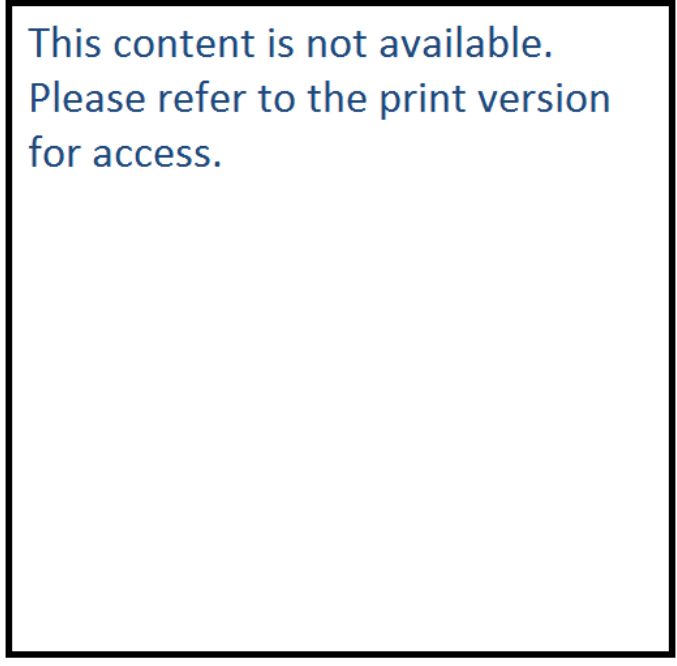

Figure 2.5: Axial-symmetric (drum) Flux Pump. Source:[39, 23].

The LTS FP operates on the principle of increasing the total flux in the superconducting loop [39, 23, 2]. This can be understood by considering a simplified version of FP consisting of a LTS thin sheet (stator) connected to the load via superconducting leads. This is shown in figure 2.6. In the high perpendicular field region the superconductivity is destroyed and a normal spot is formed. When the magnet moves from $\mathrm{A}$ to $\mathrm{B}$, the normal spot moves across the thin sheet and the current path is short-circuited by the eddy-current in the superconducting sheet. When the magnet moves from $\mathrm{B}$ to $\mathrm{C}$, a change of flux occurs in 
the closed loop consisting of the sheet, the leads and the load. Faraday's law of induction requires that EMF is induced in the closed loop. As a result a current is supplied to the load.

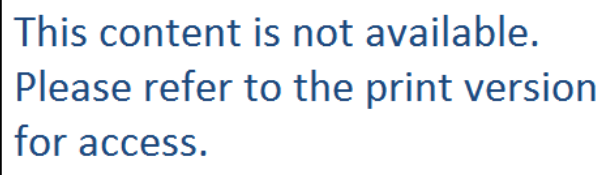

Figure 2.6: A simple LTS flux pump. Source:[39].

\subsubsection{HTS Flux Pump}

\subsubsection{The Dynamic Resistance}

FPs employing a HTS material operate differently from LTS FPs because a normal spot does not occur in the HTS material. Instead, the material in the region of high magnetic field (perpendicular) is in the mixed state as discussed before. If the material is carrying a DC transport current and a perpendicular AC magnetic field is applied to it, the transport current decays. The loss is due to the flux flow in the superconducting material caused by the interaction between the transport current and the AC field. This is known as the dynamic resistance and was studied for a slab-like tape in parallel external field [30]. For the case of perpendicular external field, the equation [19] that describes the dynamic resistance is:

$$
\frac{R_{d \perp}}{L}=\frac{4 a f}{I_{c}}\left(B_{a, \perp}-B_{t h, \perp}\right)
$$

where $B_{a, \perp}$ is the amplitude of the applied perpendicular AC field and $B_{t h, \perp}$ is the perpendicular threshold field for flux entry into the superconductor. $2 a$ is the width of the superconducting tape. $L$ is length where the field is interacting with the tape and $f$ is the frequency of the AC field. The dynamic resistance occurs when $B_{a, \perp}>B_{t h, \perp}$.

Dynamic resistance has been demonstrated to exist in mechanically rotating HTS FPs and imposed a limit on the maximum current that can be supplied by the FPs [18].

\subsubsection{The DC Voltage Generation in a HTS FP}

Although $R_{d}$ imposes a limit on the current output of a FP, it is the mechanism that is responsible for the generation of non-zero average voltage in the HTS FP. This was investigated in [12] using a travelling wave FP. It was done by applying an AC magnetic wave to a superconductor loop and this resulted in a non-zero DC voltage developed across one of the branches in the loop. 
The voltage generation mechanism was also investigated in [5] for a dynamo-type FP. In the paper, the authors reported that the shape of open-circuit voltage $\left(V_{o c}\right)$ waveform measured across the stator in the superconducting state was different from the waveform shape obtained in the normal conducting state. As a result, the time averaged output voltage in the superconducting state is not equal to zero. Their results are reproduced in figure 2.7. The plot on the left depicts the change in the shape of the AC waveform measured in the superconducting state compared with normal state. The plot on the right shows that the integration of the superconducting waveforms increases in magnitude over a few measurement cycles, whereas the average value of the normal state is always zero.
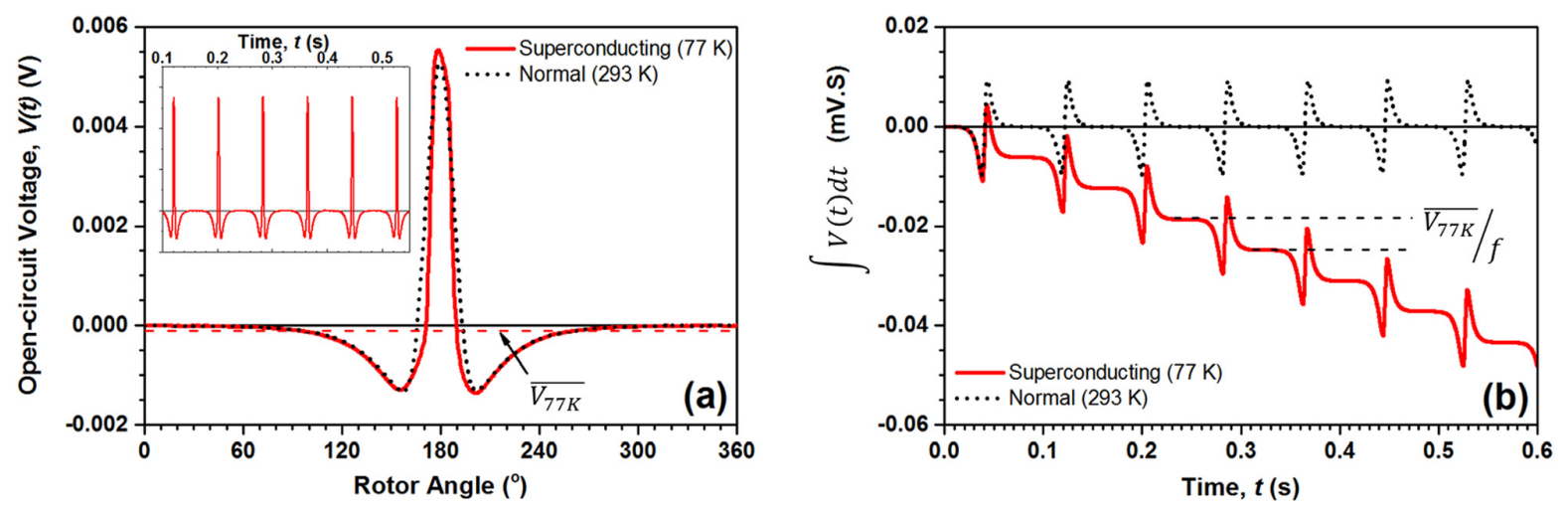

Figure 2.7: The AC waveforms of a dynamo-type HTS FP. Source:[5].

The voltage generation in the dynamo-type FP was attributed to the change in $J_{c}$ and the flux flow resistivity in the high field region. Figure 2.8 shows the situation where a square permanent magnet is moving perpendicularly across a piece of HTS tape. The red curved arrows represent the eddy current. $\boldsymbol{J}_{\text {ser }}$ and $\boldsymbol{J}_{\text {sh }}$ represent the induced current and shunt leakage current respectively. $w$ is the total width of the tape and $l$ is the length of the magnet. $\delta$ is the width where the magnet is overlapping the tape and the induced current is flowing. Finally, $w-\delta$ is the remaining width where the shunt leakage current is flowing. 


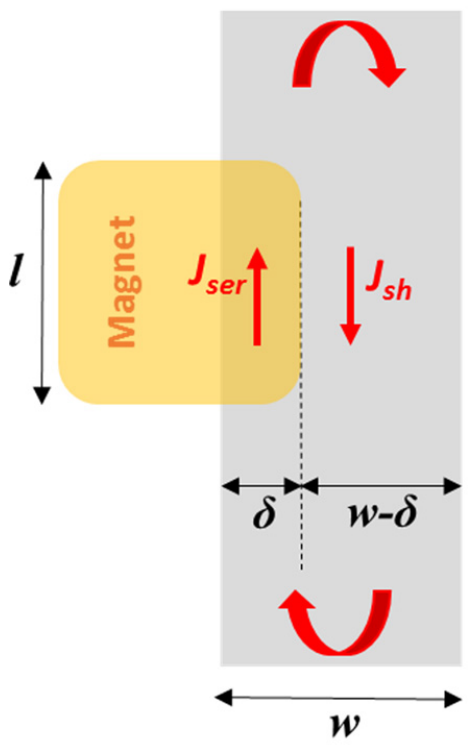

Figure 2.8: Different regions in HTS tape when a magnet is crossing over it. Source:[5].

The authors showed that, using the power law relationship, the resistance $R_{s h}$ in the shunt leakage current path can be expressed as a function of $\boldsymbol{J}_{s h}$. Similarly, the effective resistance $R_{\text {ser }}$ in the region where the current is induced can be expressed as a function of $\boldsymbol{J}_{\text {ser. }}$. The equivalent circuit is shown in figure 2.9 .

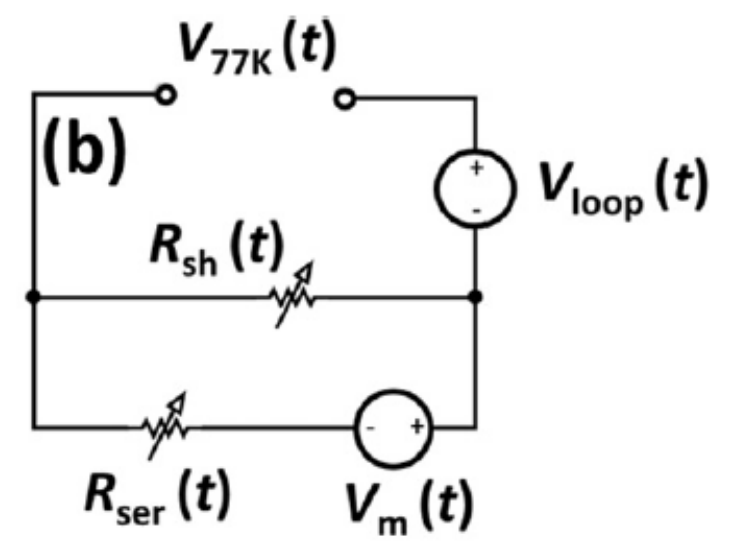

Figure 2.9: HTS Flux Pump Voltage Generation. Source:[5].

$V_{m}$ is the induced EMF in the region where the magnet and the tape overlap. $V_{77 k}$ represents the voltage measured in the superconducting state. The voltage measured in the normal state is $V_{293 K}=V_{m}+V_{\text {loop }}$, where $V_{\text {loop }}$ is the voltage picked up by the voltage measurement leads. By using the model and these relationships the authors arrived at the equation below for the non-zero DC voltage:

$$
\Delta V(t)=V_{77 K}-V_{293 K}=\frac{-V_{m}(t)}{1+\frac{R_{s h}(t)}{R_{s e r}(t)}}
$$

Furthermore, the authors showed that the resistances are related to the geometry by: 


$$
\frac{R_{s h}(t)}{R_{s e r}(t)} \approx\left(\frac{\delta(t)}{w-\delta(t)}\right)^{n}
$$

where $n$ is the constant defined in the power law relationship in equation 2.1.

Equation 2.3 implies that the non-zero average voltage is the result of the change in the resistances when the magnet is crossing over the tape.

The AC waveform changes shape because as the magnet passes the tape, the eddycurrents short circuit the EMF and partially rectifies the $\mathrm{AC}$ waveform. As a result, the average voltage is not equal to zero.

\subsection{Behaviour and the I-V Characteristics of the Flux Pump}

In the previous section, the AC behaviour of a HTS FP was discussed and the mechanism behind the non-zero average voltage generation was attributed to the change in the resistivity in the tape. This effect occurs at high frequency AC field, but gives rise to a time-averaged DC voltage measured across the stator.

For the DC behaviour of the FP, a DC equivalent circuit [17] has been proposed and it is shown in figure 2.10 .

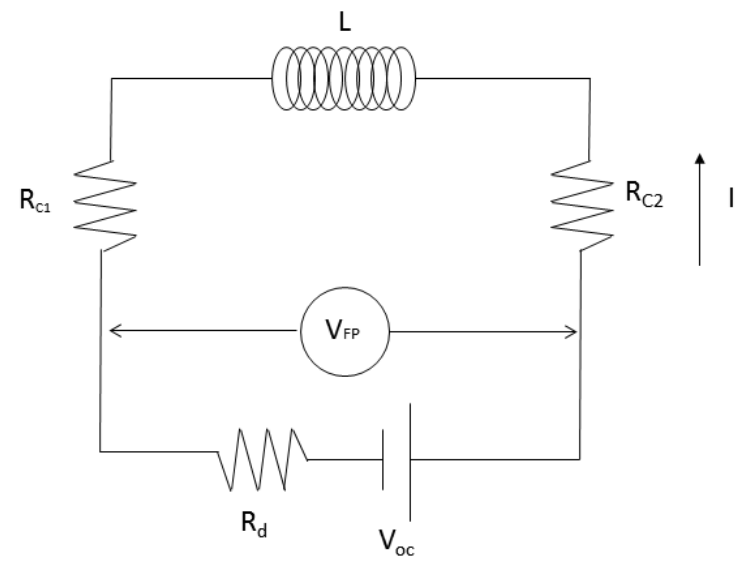

Figure 2.10: HTS Flux Pump Equivalent Circuit.

The current pumping performance of the FP is quantified by the following parameters:

1. Open-circuit Voltage, $V_{o c}$

2. Short-circuit Current, $I_{s c}$

3. Dynamic resistance, $R_{d}$

In addition, $L$ is the load of the FP (e.g. a current coil) and $R_{c 1}$ and $R_{c 2}$ are the contact resistances that occur at the solder joints. 
The $V_{o c}$ of a FP determines the maximum output voltage of the device and is obtained when the device is at no-load condition (e.g. the load is removed). When a DC current $I$ is present in the circuit, the DC output voltage $V_{F P}$ of the flux pump is described by the equation:

$$
V_{F P}=V_{o c}-I R_{d}
$$

$I_{s c}$ is the maximum current that can be supplied by the FP. If the load is a superconducting coil, it is only limited by $R_{d}$ and is described by the simple relationship:

$$
I_{s c}=\frac{V_{o c}}{R_{d}}
$$

However, in a practical situation the maximum saturation current $\left(I_{\text {sat }}\right)$ that can be produced by the FP is always lower than $I_{s c}$ due to the total solder contact resistance $R_{c}$ in the circuit. Therefore, the maximum current is:

$$
I_{s a t}=\frac{V_{o c}}{R_{d}+R_{c}}
$$

$R_{d}$ is the dynamic resistance discussed earlier. It arises from the interaction between the transport current and the $\mathrm{AC}$ magnetic field. The value of $R_{d}$ can be obtained from the inverse of the slope of current vs voltage plot ( $I-V$ curve) of the FP.

An example of the $I-V$ curve obtained from a dynamo-type HTS FP in this project is shown in figure 2.11. $V_{o c}(\approx 1.2 \mathrm{mV})$ is the x-axis intercept, $I_{s c}(\approx 95.7 \mathrm{~A})$ is the $\mathrm{y}$-axis intercept and $R_{d}(\approx 12.5 \mu \Omega)$ is the inverse $(d I / d V)^{-1}$ of the slope of the curve.

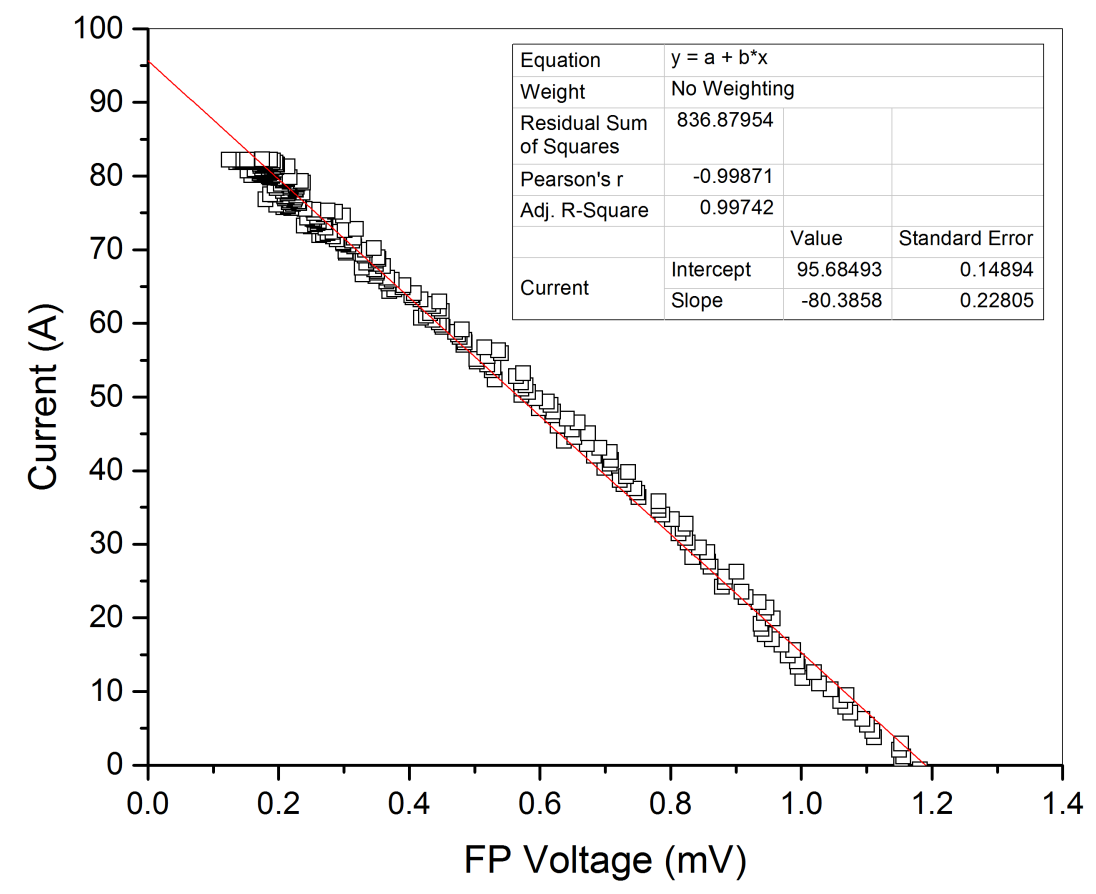

Figure 2.11: HTS FP $I-V$ curve.

The FP current plotted as a function of time is shown in figure 2.12. It shows that the 
current in the $\mathrm{FP}$ circuit reaches a maximum value at $I_{s a t}=82 \mathrm{~A}$, which is lower than $I_{s c}$ due to the total solder contact resistance in the circuit.

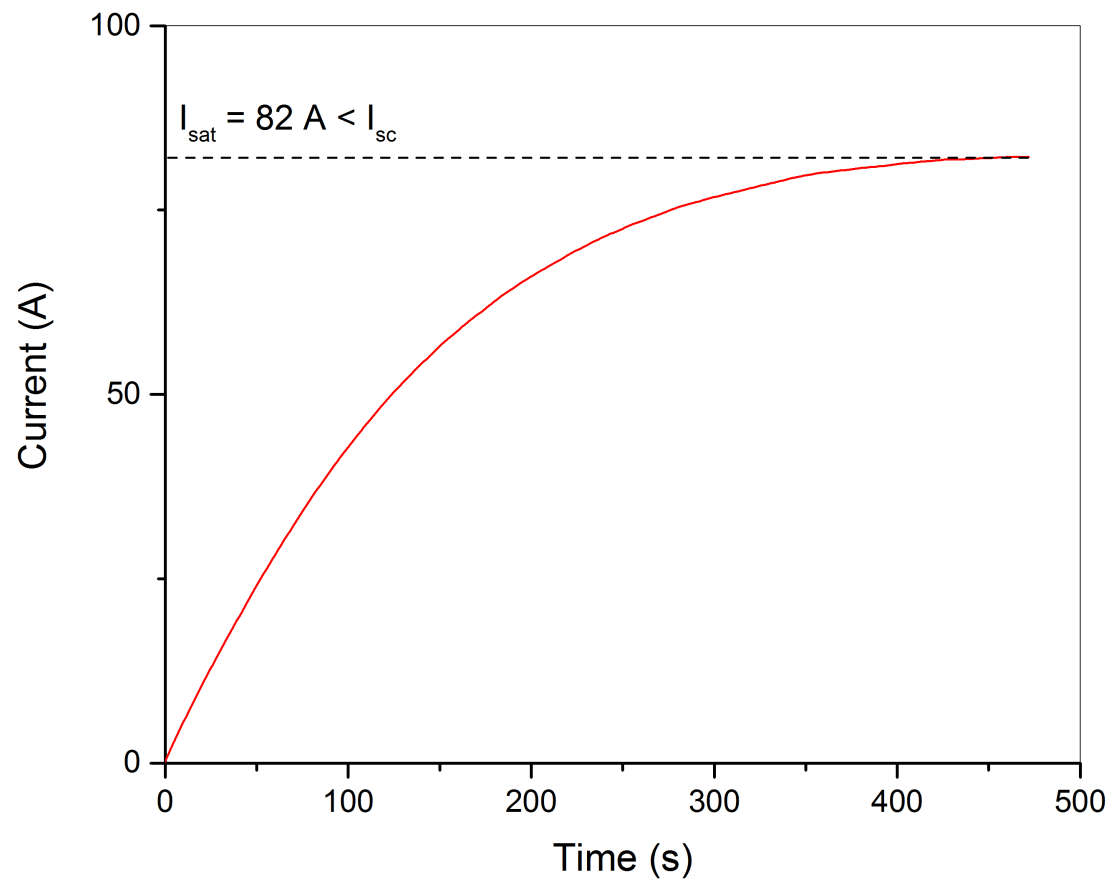

Figure 2.12: HTS FP current vs time plot.

In the following chapters, the design and construction of the FPs used in this project will be discussed. The parameters $V_{o c}, R_{d}$ and $I_{s c}$ are used throughout this thesis to describe the performance of the FPs. The results of the experiments conducted using the FPs will be analysed and the $I-V$ curves will be used to obtained the performance parameters. 


\section{Chapter 3}

\section{Gen 1 and Gen 2 FP Systems Experimental Designs}

Two existing experimental systems have been employed in this project to investigate the performance of HTS FPs. The first system was used to study the effect of the geometry of the rotor permanent magnet on the performance of FPs. It is known as the first generation (G1) FP system and was cooled to $77 \mathrm{~K}$ by liquid nitrogen $\left(N_{2}\right)$. The second system was used to study the frequency dependent behaviour of the FPs and the effect of the width of the stators on the FP performance. It is identified as the second generation (G2) FP system and was also cooled to $77 \mathrm{~K}$ by liquid $N_{2}$.

\subsection{RRI First Generation Flux Pump (Gen 1)}

\subsubsection{HTS Stator and Mechanical Construction}

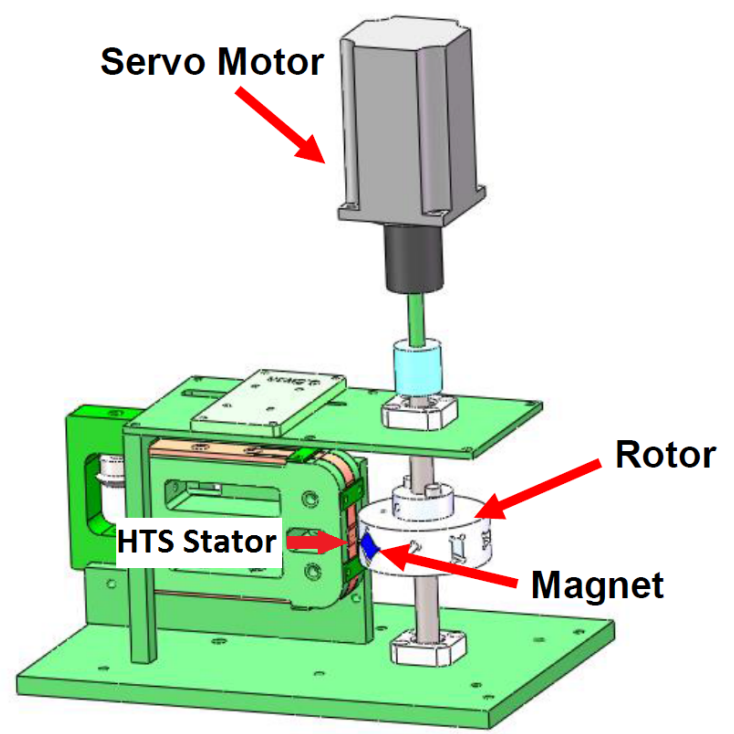

Figure 3.1: The arrangement of the Generation 1 HTS FP system. Source:[3]. 
The Robinson Research Institute first generation FP (Gen 1) system is shown in figure 3.1. The HTS stator is made of a piece of $12 \mathrm{~mm}$ wide copper-coated YBCO HTS conductor supplied by SuperPower. It is held in a vertical orientation by a G10 holder. The stator is positioned so that it is always parallel to the rotor rotational axis (see figure 2.4). The horizontal position of the G10 holder can be adjusted to achieve the desired flux gap between the stator and the magnet.

The ends of the stator are connected to normal-conducting copper current leads. In this project, instead of connecting the FP to a load (e.g. a HTS current coil), it was connected to a constant current supply to drive the current in the HTS circuit. It has been demonstrated that this method is equivalent to connecting the FP to a current coil [4]. This is demonstrated in figure 3.2 which shows that the I-V curves obtained from the FP using a power supply are equivalent to the I-V curves of the FP using a current coil. The advantage of using the power supply is that it allows for much faster scanning of the voltage and current outputs from the FP.

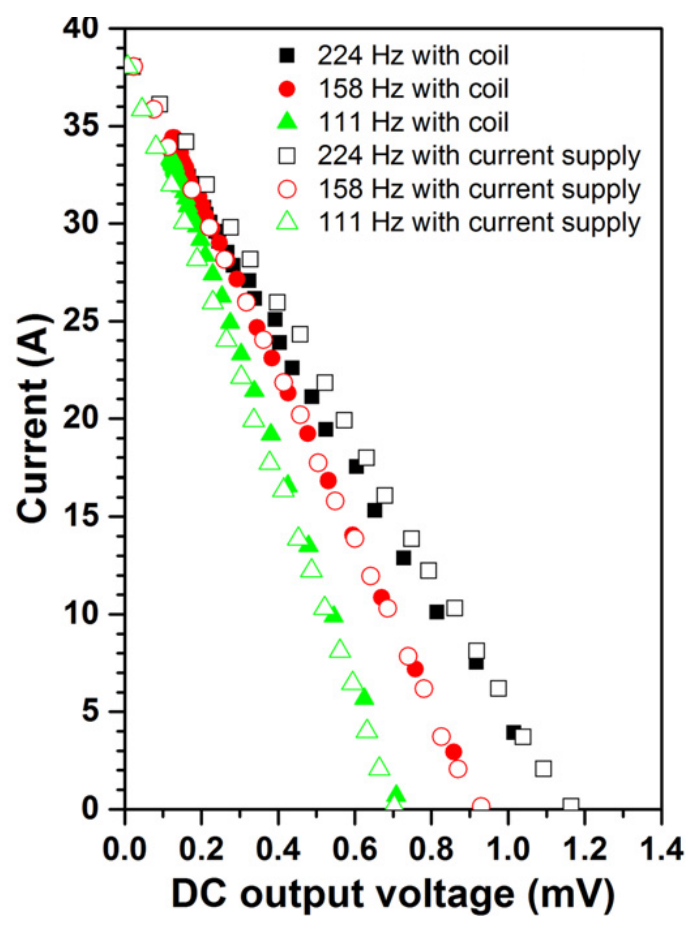

Figure 3.2: FP $I-V$ curves measured at 3 different frequencies using two different methods. Source: [4].

The rotor (cylindrical shape) of the Gen $1 \mathrm{FP}$ is made of aluminium and holds permanent magnets (N42 Nd-Fe-B type) of different sizes and orientation. The rotor is coupled to a shaft driven by an Omron servo motor. The rotation speed and direction of the rotor are controlled by a National Instrument output module and Labview software.

Apart from the motor, the whole unit is submerged in a liquid $N_{2}$ bath. 


\subsubsection{Schematic Diagram and Signal Acquisition}

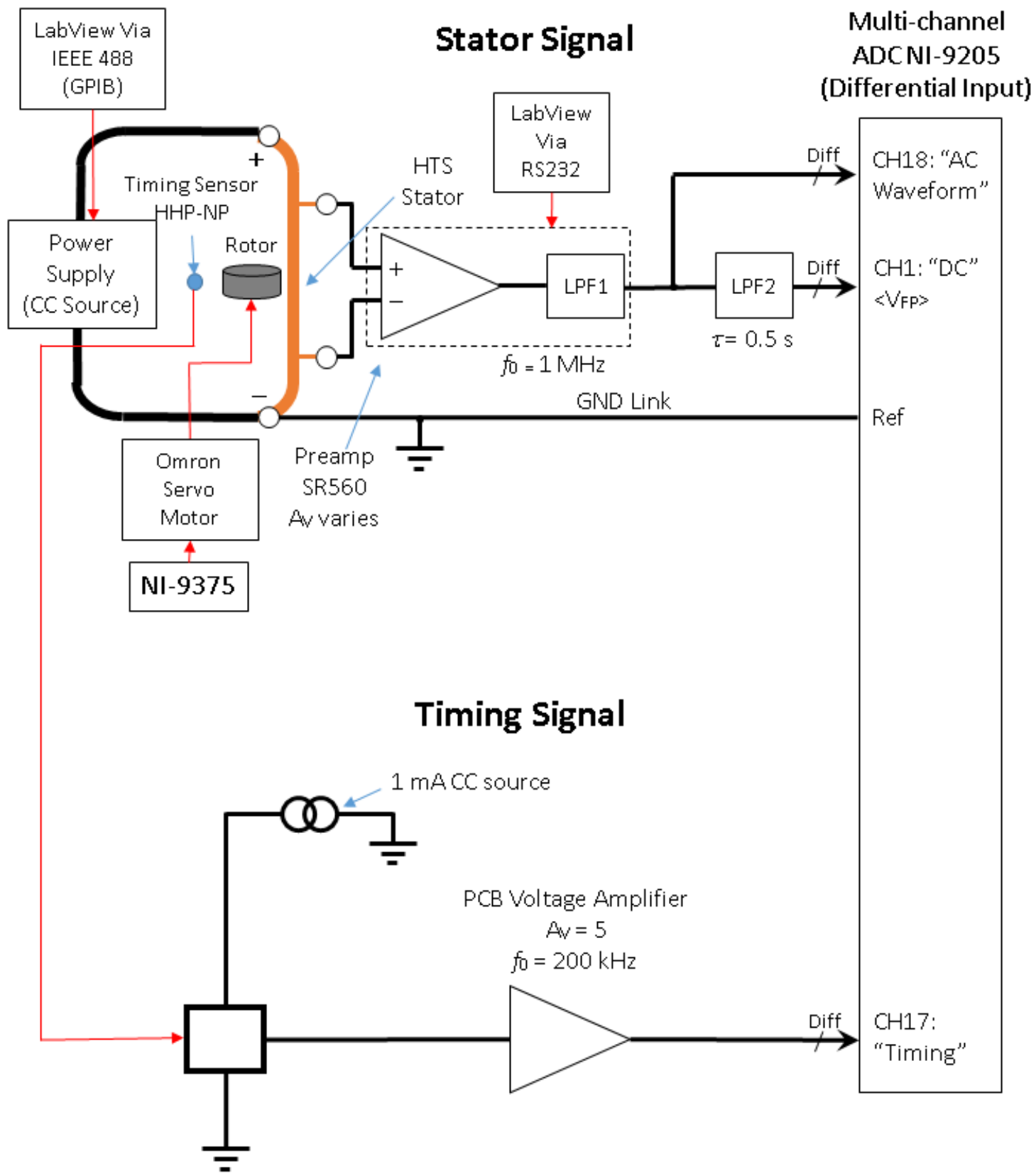

Figure 3.3: Gen 1 FP system schematic and signal acquisition. CC: Constant current source, GPIB: General Purpose Interface Bus, LPF: Low pass filter, Diff: Differential voltage input, $A_{V}$ : Voltage gain, $f_{0}$ : cut-off frequency, $\tau$ : Time constant. Modified from the original diagram supplied by A. E. Pantoja, RRI.

Figure 3.3 shows the schematic diagram of the G1 FP system and the DAC acquisition units. The HTS stator and the timing voltage signals were measured using the National Instrument multi-channel NI-9205 analogue-to-digital converter. The high resolution and sampling rate features of the device were crucial for the AC measurements. The sampling rate was adjusted so that every 10 cycles of the magnet rotor gave 9600 data points. The differential mode was 
chosen for the measurements due to the low signal level (in the range of $\mathrm{mV}$ and $\mu \mathrm{V}$ ) and long signal lead length. Twisted-pair signal leads with ground shield were used whenever it was practical.

The HTS voltage was measured and amplified by the low-noise preamplifier SR560. The voltage gain and the low-pass-filter (LPF) cut-off frequency were controlled by the LabView software. The pre-amplified signal was then split into two channels, one was fed directly to the NI-9205 to record the AC voltage waveform. The other channel of the pre-amplified signal was fed to the LPF (noise filtering) and this DC signal was used for the real-time data display and monitor in the acquisition software.

The position of the magnet on the rotor with respect to the stator was detected by a Hall sensor. For every cycle of the rotor rotation, the voltage signal output of the Hall sensor varied according to the magnetic field strength it detected. Similar to the stator voltage signal, the Hall sensor signal was fed to a pre-amplifier and a LPF (noise reduction) before it was acquired by the NI-9205.

In this project, a power supply (Agilent Technologies 6970 A) was used to supply a constant current in the superconducting circuit. The rotor was spun at a constant frequency and the power supply current was increased from zero to a maximum value at a fixed current step (typically 2A) and at the same time the stator voltage was measured. This method allows for a much faster construction of the I-V curve compared to using a HTS current coil.

\subsection{RRI Second Generation Flux Pump (Gen 2)}

\subsubsection{HTS Stator and Mechanical Construction}

As discussed in section 2.3, the main advantage of a FP powering an HTS current coil compared with a conventional power supply is that it does not require wire connections between the power source and the HTS load. This greatly reduces the heat load of the cryogenics system. However, a FP system (such as the Gen 1 system) that is completely submerged in the liquid $N_{2}$ bath still suffers the heat load associated with the moving parts (rotor, rotor shaft and bearings) and coupling shaft to the motor, which is located at room temperature. In order to eliminate these heat loads the moving parts can be located outside of the cryogenic environment. This has been achieved by the RRI second generation (Gen 2 )FP system and it is shown in figure 3.4. 


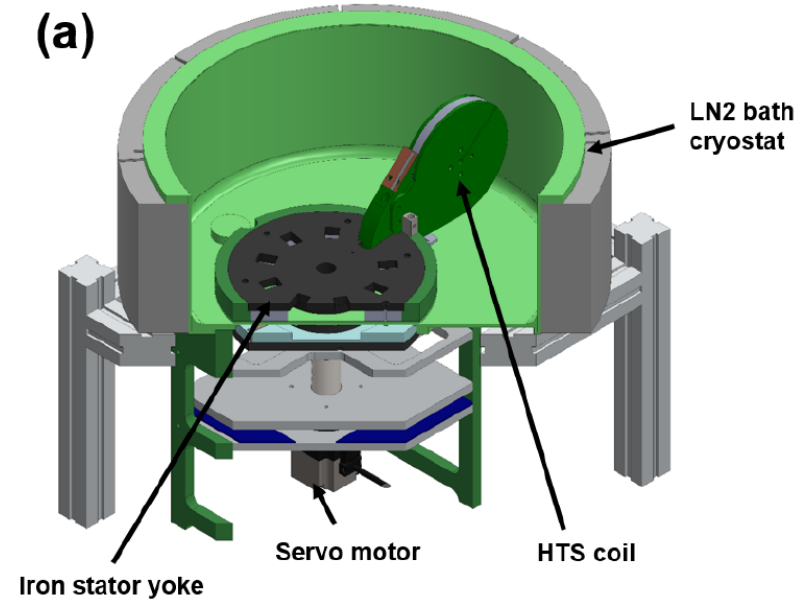

(a) System construction

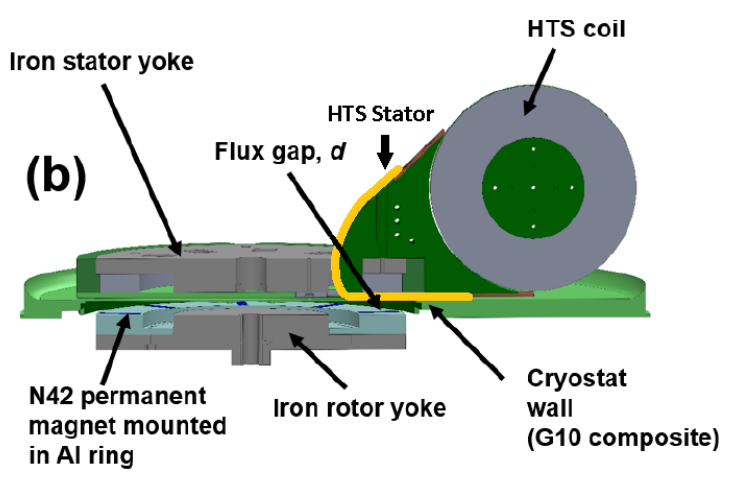

(b) Cross-section view

Figure 3.4: The arrangement of the generation 2 FP system. Source:[6].

In the liquid $N_{2}$ bath cryostat, the HTS stator is mounted on an iron yoke which has the capacity to accommodate nine individual stators. Each of the stators has a maximum width of $46 \mathrm{~mm}$ and is connected to a HTS double pancake coil via normal conducting copper pieces. The inductance of the coil is $1.97 \mathrm{mH}$ and the $I_{c}$ is $95 \mathrm{~A}$, measured at $1 \mu \mathrm{V} / \mathrm{cm}$ criteria. In this project, one stator was used in the experiments.

Outside the liquid $N_{2}$ bath cryostat, an iron rotor yoke is located under the stator yoke (see figure $3.4 \mathrm{~b}$ ). They both have a common axis. The rotor has the capacity to hold up to nine N42 type permanent magnets and is driven by an Omron servo motor. In this project, nine magnets were used in the experiments so that in a full rotation cycle nine magnets will have crossed over the HTS stator. This implies that the G2 FP operating frequency is nine time higher compared with the G1 FP.

The stator and rotor yokes act as a magnetic path and form a complete magnetic circuit, which concentrates flux onto the HTS stator.

In this arrangement, the HTS stator and current coil form a complete superconducting circuit and are located in the liquid $N_{2}$ bath at $77 \mathrm{~K}$. On the other hand, the moving parts (rotor, coupling shaft and motor), which are located outside the cryostat, do not introduce heat load to the cryogenics system. Another advantage of the $2 \mathrm{G} \mathrm{FP}$ system is that, due to high concentration of flux on the HTS stator, it allows for a large flux gap between the permanent magnets and the cryostat wall. Current injection has been achieved at flux gap of up to $14.5 \mathrm{~mm}$ [6]. This implies that a thick insulating material can be used to insulate the cryostat wall. 


\subsubsection{Schematic Diagram and Signal Acquisition}

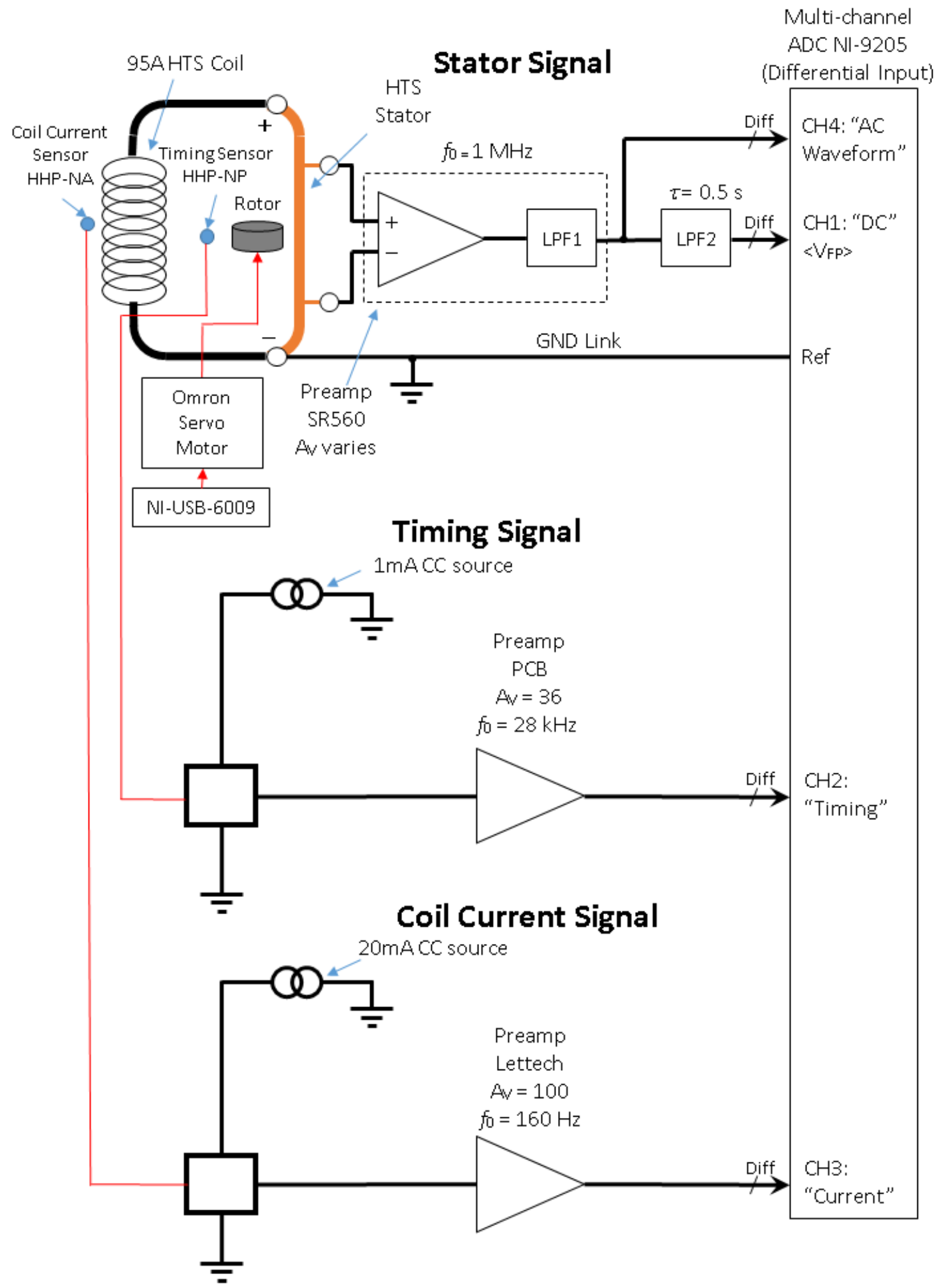

Figure 3.5: Gen 2 FP system schematic and signal acquisition. CC: Constant current source, LPF: Low pass filter, Diff: Differential voltage input, $A_{V}$ : Voltage gain, $f_{0}$ : cut-off frequency, $\tau$ : Time constant. Modified from the original diagram supplied by A. E. Pantoja, RRI.

Figure 3.5 shows the schematic diagram of the G2 FP system. The overall construction is very similar to the G1 system, except that the constant current supply was replaced by a 
HTS current coil. Similar to the G1 system, the stator voltage in the G2 FP was measured by the SR560 low-noise preamplifier. This signal was fed directly to the NI-9205 acquisition unit for the $\mathrm{AC}$ waveform and also via the LPF to a separate channel for real-time display of the I-V curve.

The arrangement of timing signal acquisition is also similar to the G1 system. However, in the G2 system the rotor held nine equally spaced magnets. Therefore, the timing signal had nine peaks, each of them indicated the time when one of the magnets was located at the centre the stator.

The HTS current coil and the HTS stator were connected in series. A calibrated Hall sensor was placed at the centre of the coil to detect the current in the circuit. The existing calibration curve of the Hall sensor and the coil arrangement was used in the experiments. 


\section{Chapter 4}

\section{Gen 1 and Gen 2 FP Systems Experimental Results}

\subsection{Gen 1 Flux Pump System Experimental Results}

The first generation (Gen 1) flux pump system has been used in this project to investigate the pumping performance and the characteristics of the HTS dynamo-type FP. The objective of the experiments was to investigate the effect of the shape, size and orientation of the rotor permanent magnet on the FP performance. First, the setup of the magnets and the material used for the HTS stator are discussed. After that, an example of the AC waveforms measured across the stator at superconducting state is analysed. The mechanism that is responsible for the non-zero average voltage output of the stator is then discussed. This is followed by the results for the $I-V$ curves and the FP performances $\left(V_{o c}, R_{d}\right.$ and $\left.I_{s c}\right)$ produced with different magnet geometries. Finally, some design rules for optimising the FP performance are discussed.

An existing Gen 1 experimental system was used to run the experiments. However, new FP stators were made from superconducting tapes and new set of magnets were installed for the project. Some of the results and discussions presented in this section have been published in IEEE Transactions on Applied Superconductivity [3].

\subsubsection{Experiment Samples Setup}

Three different types of magnet shapes (square and rectangular) and sizes $(12 \times 12 \mathrm{~mm}$, $6 \times 12 \mathrm{~mm}$ and $3 \times 12 \mathrm{~mm}$ ) were investigated in the experiments. The orientations of the magnets with respect to the HTS stators were as follows:

1. Vertical $\left(0^{\circ}\right)$ - Magnet parallel to HTS tape

2. Angled $\left(45^{\circ}\right)$ - Magnet is at $45^{\circ}$ with respect to HTS tape

3. Horizontal $\left(90^{\circ}\right)$ - Magnet is at $90^{\circ}$ with respect to HTS tape. 
The combinations are summarised in table 4.1.

\begin{tabular}{|c|c|c|}
\hline Magnet Orientation and Width & $3 \mathrm{~mm}$ or $6 \mathrm{~mm}$ & $12 \mathrm{~mm}$ \\
\hline \hline & nrs & \\
Vertical $\left(0^{\circ}\right)$ & în \\
& & \\
\hline Angled $\left(45^{\circ}\right)$ & & \\
\hline & & \\
Horizontal $\left(90^{\circ}\right)$ & & \\
\hline & & N/A \\
\hline
\end{tabular}

Table 4.1: Plane (x-y) view showing themagnet and HTS stator orientation.

The different magnet orientations produced different magnetic field profiles in the $\mathrm{x}$ and y directions (see the Opera Finite Element Analysis Software simulations in figures 4.6, 4.10 and 4.12). This allows for the study of the impact of the magnet geometry, with respect to the length and width of the stator, on the flux pump performance.

The HTS stators were made from SuperPower (SF12050) copper-coated YBCO tapes of $12 \mathrm{~mm}$ in width [35]. The $I_{c}$ of the tape was about $300 \mathrm{~A}$, which was larger than the expected maximum short-circuit current $\left(I_{s c}\right)$ of the FP. The rotor permanent magnets were N42 Nd-Fe-B type. The flux gap, measured from the surface of the stator to the surface of the magnet, was $3.7 \mathrm{~mm}$.

\subsubsection{The AC Waveforms and the Open-Circuit Voltage Generation}

It was discussed in chapter 2 that the shape of the AC waveform measured across a HTS stator in superconducting state is different from the waveform measured in normal conducting state. This results in the non-zero average voltage generation of the FP. An example of the frequency-normalised open-circuit AC waveforms of this experiment is shown in figure 4.1a. It was measured across the stator at $77 \mathrm{~K}$ in a liquid $\mathrm{N}_{2}$ bath. The rotor permanent magnet width was $3 \mathrm{~mm}$ and the rotor was operated at $3 \mathrm{~Hz}$ in the reverse direction. At rotor angle $180^{\circ}$ the magnet was located at the centre of the HTS stator. This is marked by the letter $\mathrm{M}$ in the figure. The normal-conducting $\mathrm{AC}$ waveform measured at room temperature 300 $\mathrm{K}$ is also included for reference. It can seen clearly that the $77 \mathrm{~K}$ and $300 \mathrm{~K}$ waveforms have different shapes. Figure $4.1 \mathrm{~b}$ shows that the integration of the $77 \mathrm{~K}$ waveform over a full rotor cycle is about $3 \mu \mathrm{V} / \mathrm{Hz}$, whereas for the $300 \mathrm{~K}$ waveform the result is zero.

In order to eliminate the effect of the voltage picked up by the remainder of the voltage loop (e.g. the voltage tap leads) the $300 \mathrm{~K}$ waveform was subtracted from the $77 \mathrm{~K}$ waveform. The result is the $\Delta V / f$ waveform shown in figure 4.1c. It shows that the voltage generation occurred when the magnet was passing over the HTS stator. 


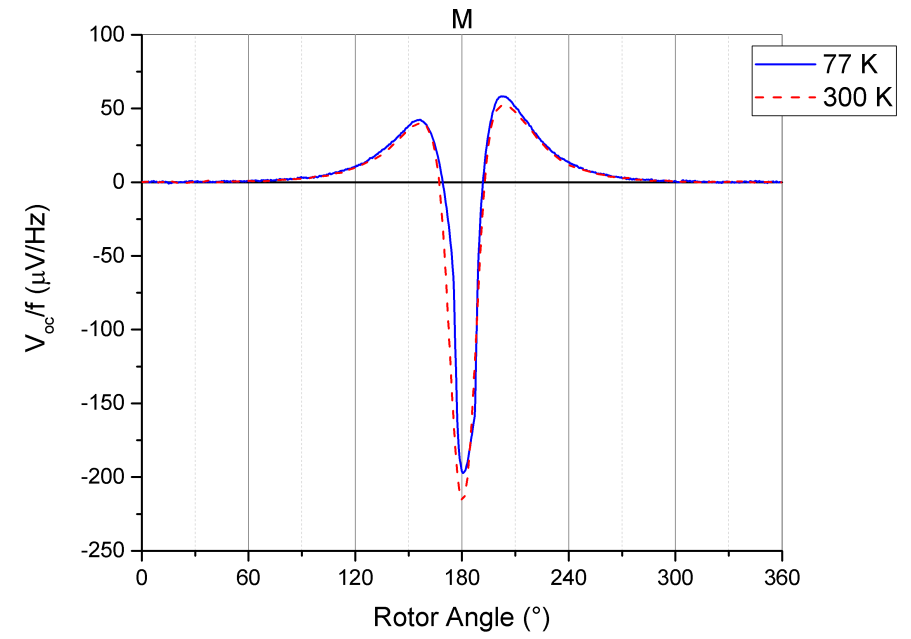

(a) $77 \mathrm{~K} \& 300 \mathrm{~K}$ AC waveforms

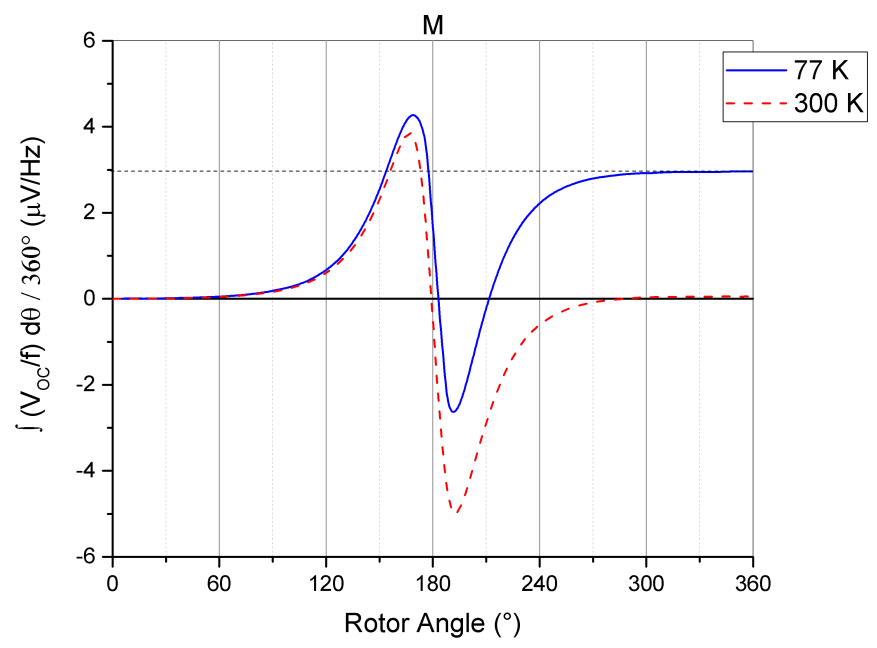

(b) AC waveform integrations

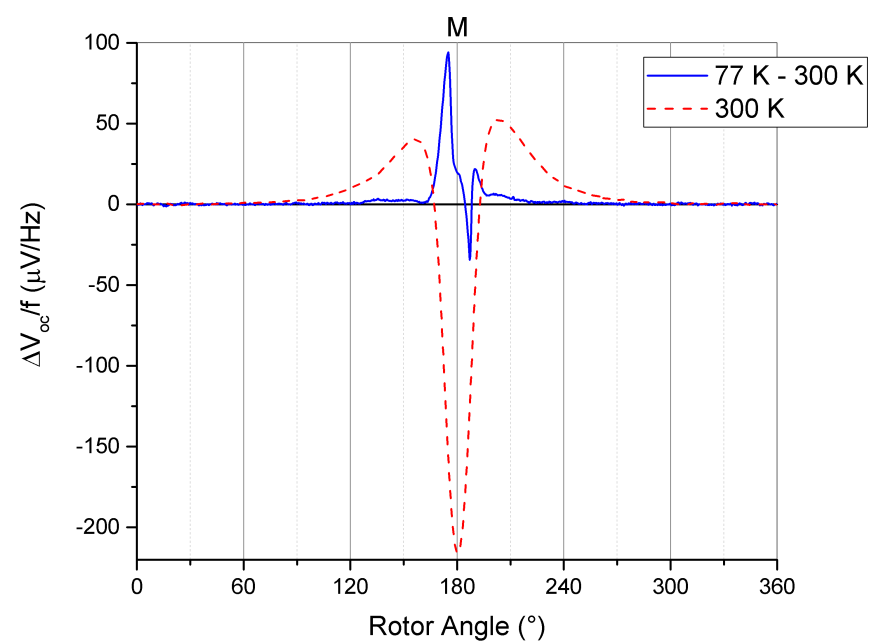

(c) $\Delta V / f$

Figure 4.1: Figures showing the $\mathrm{AC}$ waveforms of the $\mathrm{FP}$ using the $3 \mathrm{~mm}$ magnet. The magnet was in the vertical orientation with respect to the stator. The rotor was rotating in the reverse direction at $3 \mathrm{~Hz}$. 


\subsubsection{FP Performance (6 $\mathrm{mm}$ magnet, vertical orientation)}

The three parameters that measure the performance of a FP are the open-circuit voltage $V_{o c}$, the dynamic resistance $R_{d}$ and the short-circuit current $I_{s c}$. These parameters form the basis of the DC model (see figure 2.10 in chapter 2) of a FP. They can be determined from the plot of the FP current vs voltage known as the $I-V$ curve.

Figure 4.2 shows the $I-V$ curves obtained from the FP employing the 6 -mm wide magnet at vertical orientation. In this set of data the rotor was driven in the reverse direction and each of the curves were obtained at various magnet frequencies. The x-axis intercepts of the curves are the open-circuit voltages $\left(V_{o c}\right)$ and the y-axis intercepts are the short-circuit currents. The dynamic resistances $\left(R_{d}\right)$ are the inverse of the slopes. For example the $V_{o c}, I_{s c} \& R_{d}$ for $24 \mathrm{~Hz}$ Rev direction are about $114 \mu \mathrm{V}, 55 \mathrm{~A}$ and $2 \mu \Omega$ respectively. Note that the parameters $V_{o c}$ and $R_{d}$ were obtained from the line fit, whereas $I_{s c}$ was derived from $V_{o c}$ and $R_{d}$, i.e. $I_{s c}=V_{o c} / R_{d}$.

In the Gen $1 \mathrm{FP}$ experiments, another set of data were also obtained by driving the rotor in the opposite (forward) direction. The FP performance parameters of the forward and reverse directions were slightly different due to small misalignment of the stator and rotor axes.. The results of these two set of data are included in future plots for comparison.

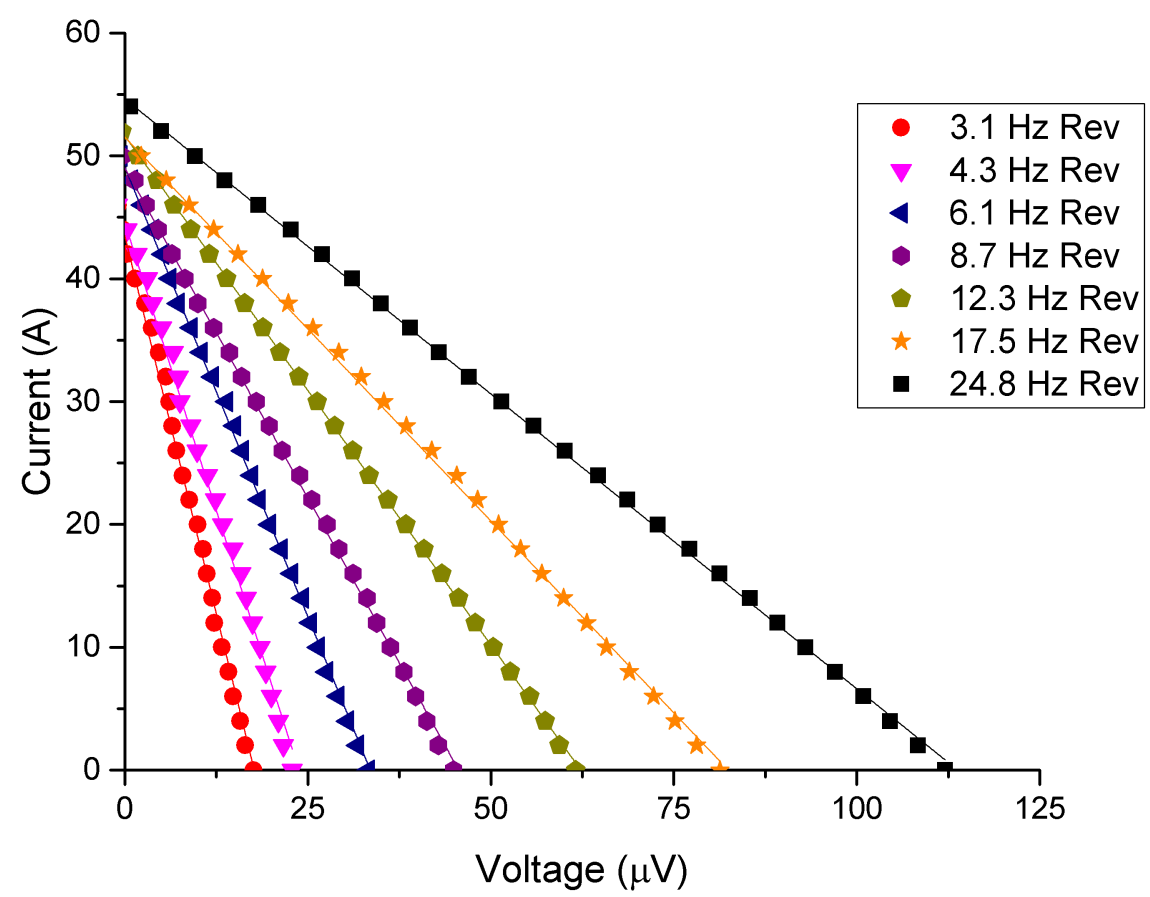

Figure 4.2: Figure showing the $I-V$ curves of the FP using the $6 \mathrm{~mm}$ magnet. The magnet orientation with respect to the stator was vertical. The lines drawn are the linear line fits.

The $V_{o c}$ is the voltage measured across the stator without any load and this is the maximum voltage that can be supplied by the FP at a fixed frequency. The $V_{o c}$ plot in figure 4.3 for both the reverse (rev) and forward (fwd) rotor rotations shows that it is increasing with 
frequency. However, the data exhibits very small non-linearity with respect to frequency and this has been reported previously in [14] and was attributed to the eddy-current loss in the copper layer of the superconducting tape.

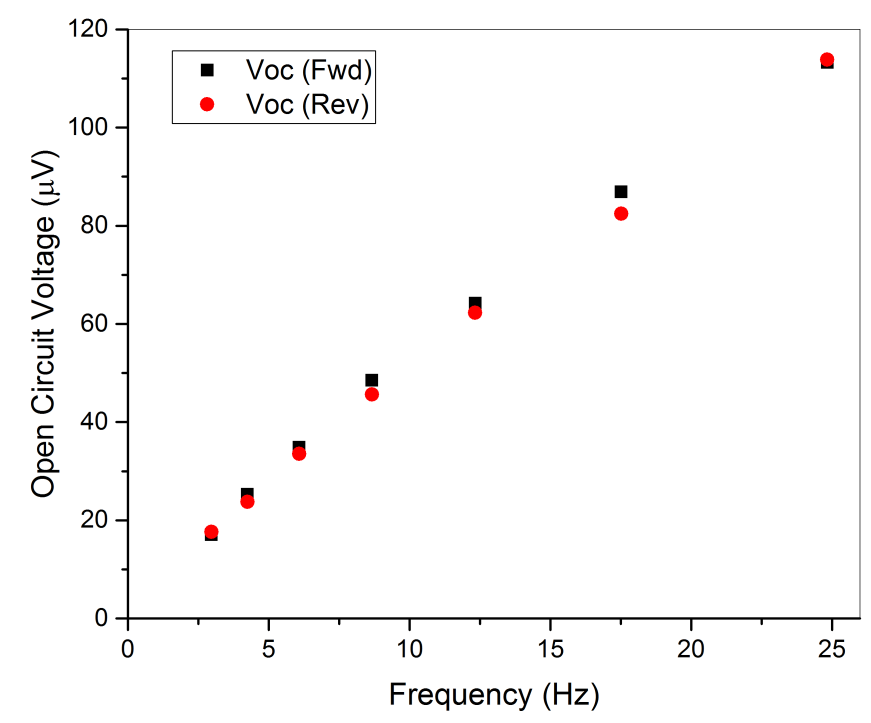

Figure 4.3: FP open-circuit voltage, $6 \mathrm{~mm}$ magnet, vertical orientation.

In order to compare the effect of frequency on the data, $V_{o c}$ and $R_{d}$ were normalised by the frequency. The normalised results $V_{o c} / f$ and $R_{d} / f$ were then plotted as a function of frequency as shown in figure 4.4. The short-circuit current plots are also included in the figure. Note that the result for forward and reverse directions are slightly different. Also note that the cross-over of the $V_{o c / f}$ at the lowest frequency. This also occurs in measurements made with different magnet shapes and orientations. In general, the difference between the output obtained for forwards and backwards operation is believed to be due to small misalignment of the stator and rotor axes. 


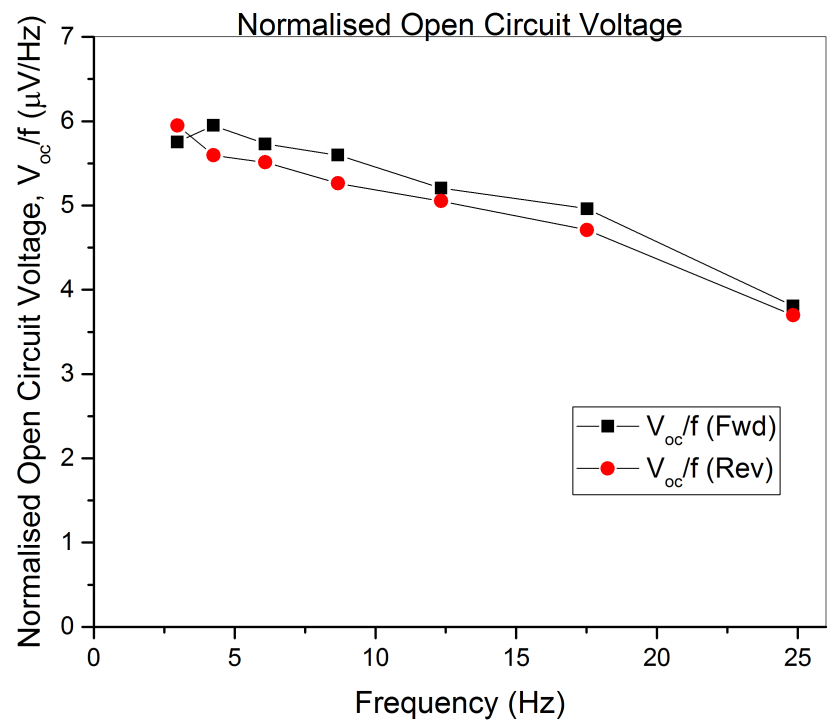

(a) $V o c / f$

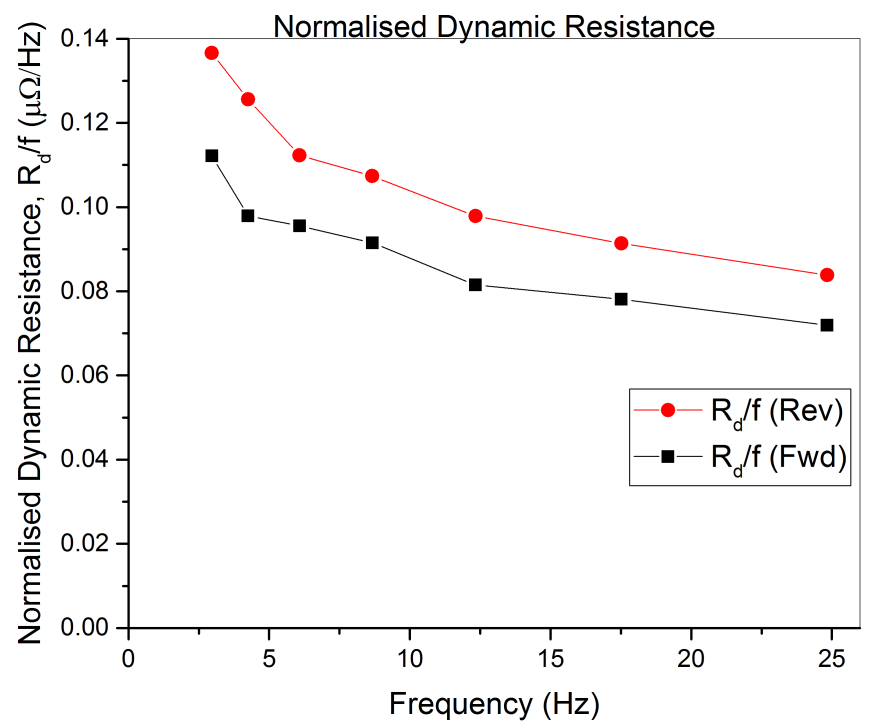

(b) $R_{d} / f$

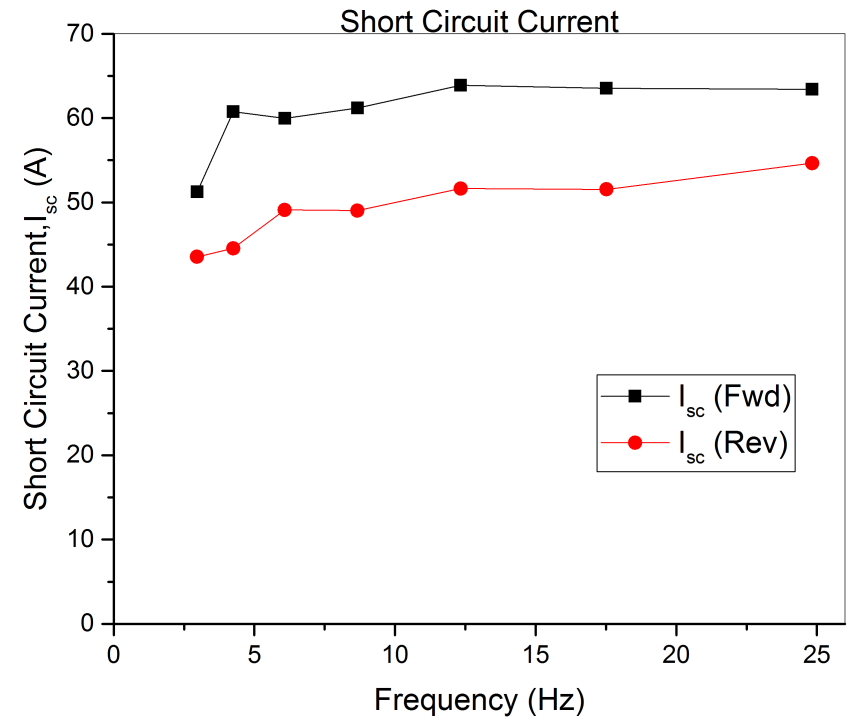

(c) $I_{s c}$

Figure 4.4: Normalised $V_{o c}$, normalised $R_{d}$ and $I_{s c}$ of Gen 1 system using $6 \mathrm{~mm}$ magnet at vertical orientation. The lines joining the data points are guides to the eye. 
Similar results were also obtained for other magnet sizes and orientations. It is instructive to compare them with the results of the $6 \mathrm{~mm}$ vertical magnet.

\subsubsection{Vertical Orientation}

Figure 4.5 shows the results of the vertical orientation for all magnet sizes. The high frequency data (17 and $25 \mathrm{~Hz}$ ) for the $12 \mathrm{~mm}$ magnet is not available for comparison. 


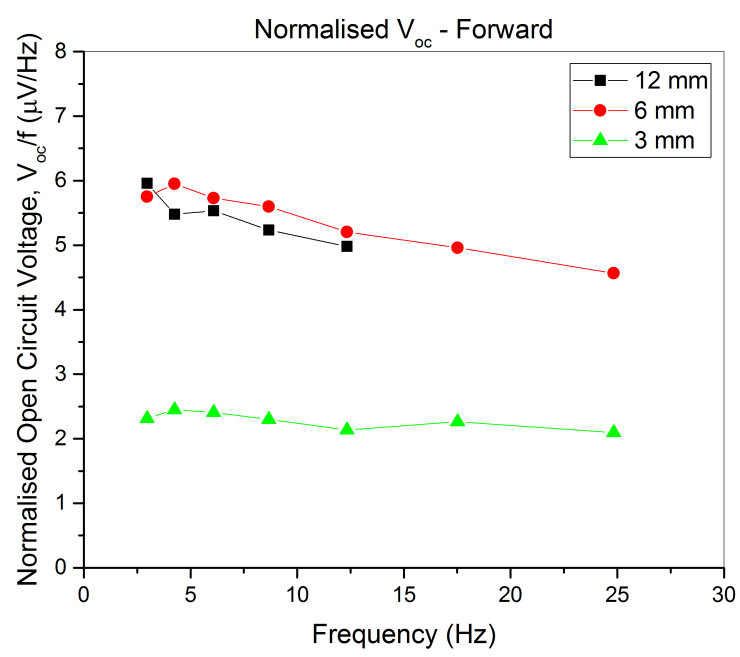

(a) $V_{o c} / f$, Forward

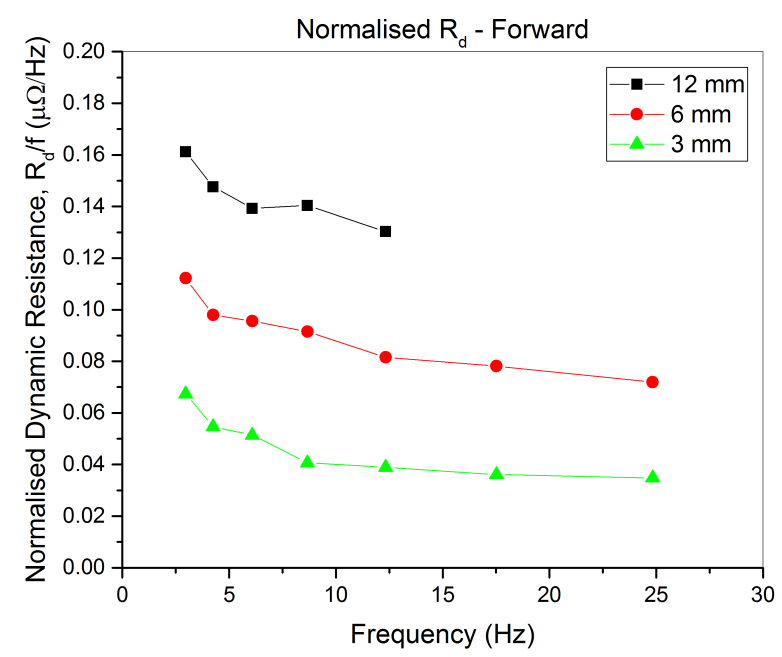

(c) $R_{d} / f$, Forward

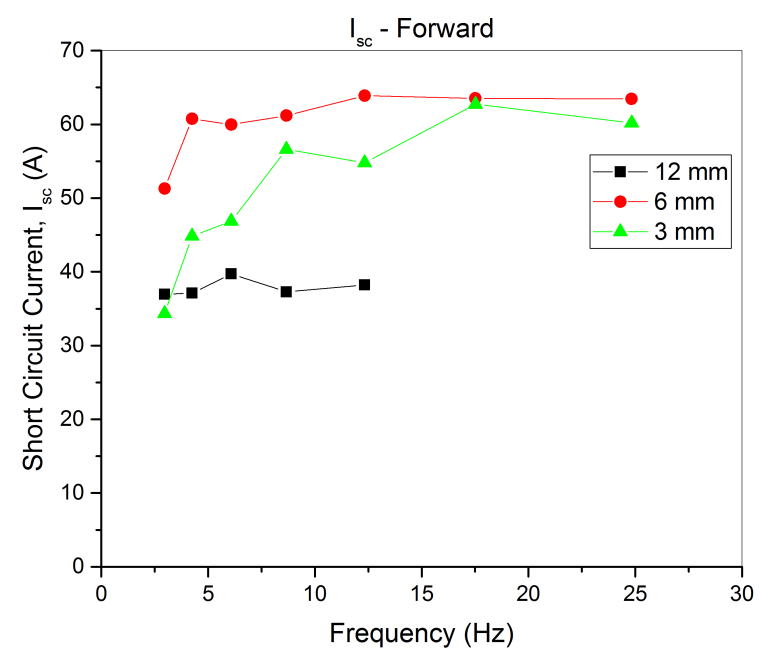

(e) $I_{s c}$, Forward

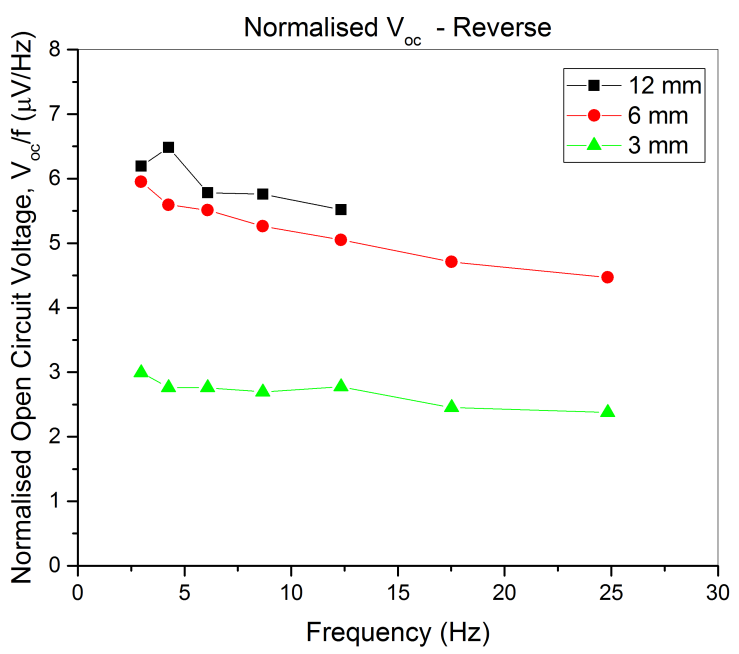

(b) $V_{o c} / f$, Reverse

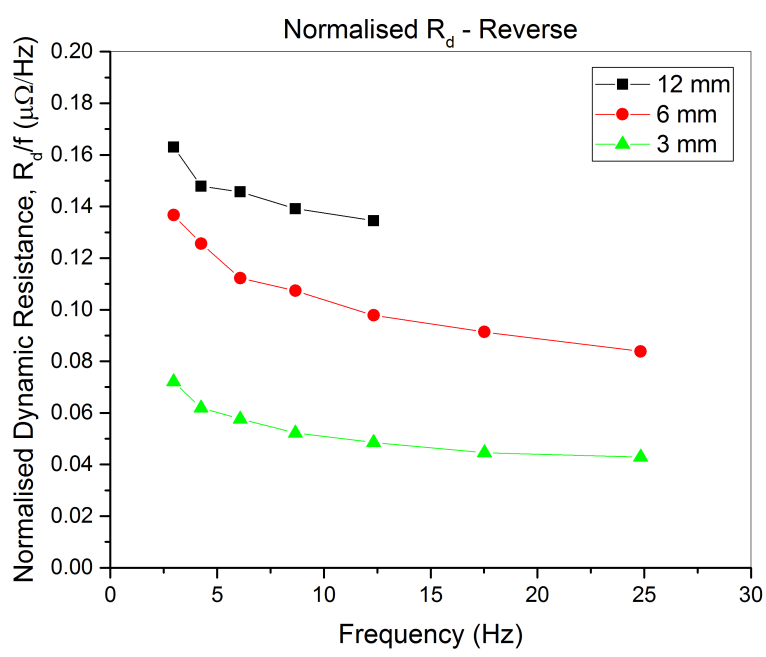

(d) $R_{d} / f$, Reverse

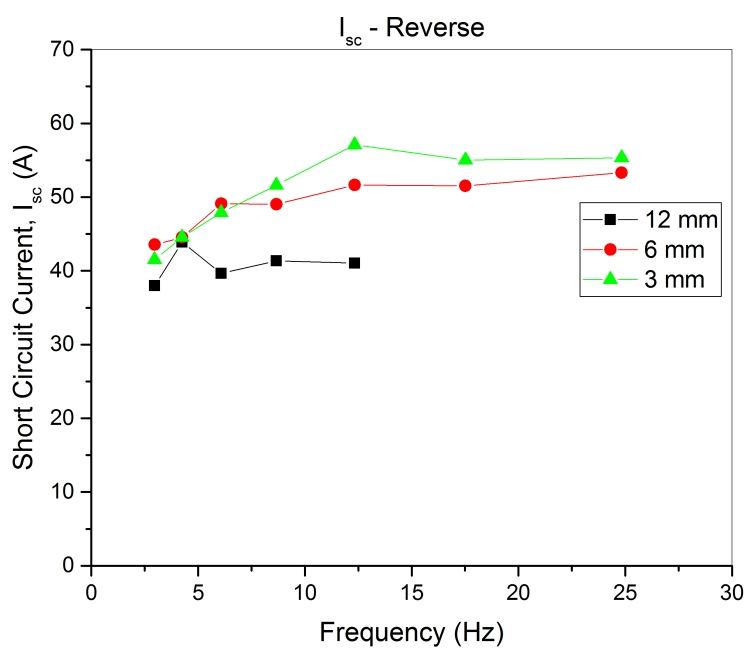

(f) $I_{s c}$, Reverse

Figure 4.5: Gen $1 \mathrm{FP}$ performance using $3 \mathrm{~mm}, 6 \mathrm{~mm} \& 12 \mathrm{~mm}$ magnets at vertical orientation. The lines joining the data points are guides to the eye. The forward data has been published in [3] and the reverse data is included for comparison.

The magnetic field profile at the stator for each of the magnet sizes was simulated using 
Opera Finite Element Analysis Software. The results are shown in figure 4.6.

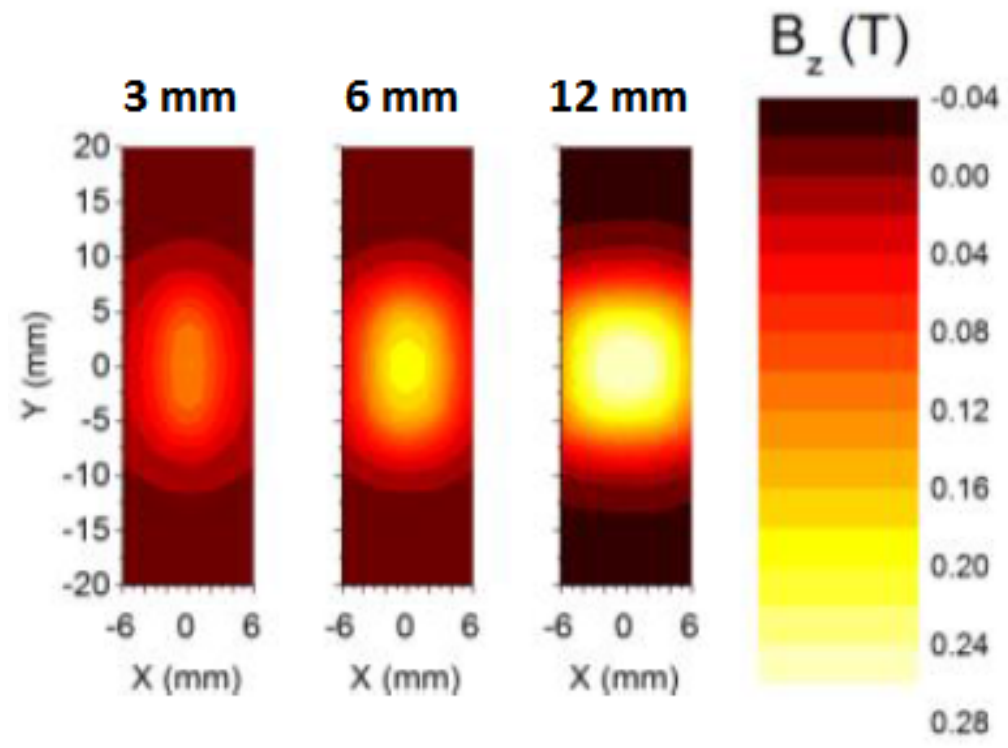

Figure 4.6: Simulated stator magnetic field profile, vertical orientation. The parameter $\mathrm{x}$ is the length measured along the width of the stator. The parameter $y$ is the length measured along the length of the stator. The flux gap is $3.7 \mathrm{~mm}$. Source:[3]

The plots for the simulated perpendicular field $B_{Z}$ for all magnet sizes and orientations are shown in figure 4.7. The plots show that the field strength increases with magnet size and the peak field strength is the same for all magnet orientations.

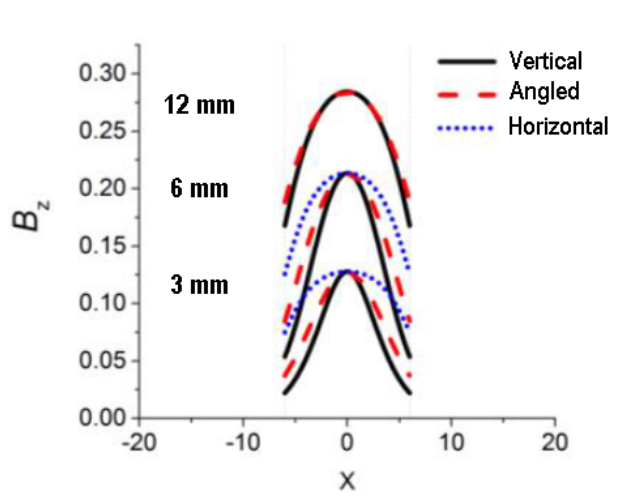

(a) $B_{z}$ along the $\mathrm{x}$ direction of the stator.

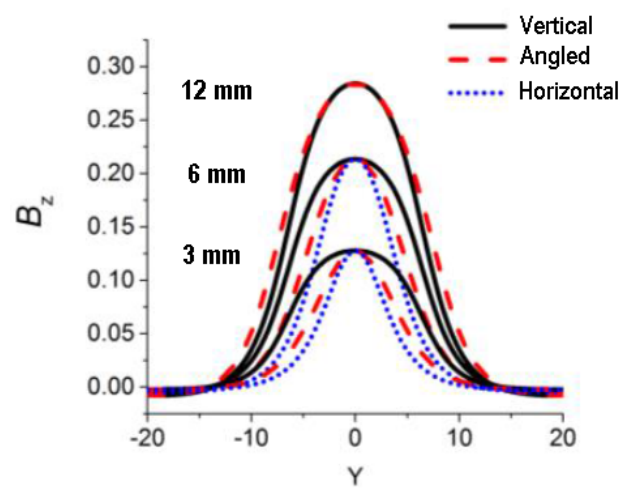

(b) $B_{z}$ along the y direction of the stator.

Figure 4.7: Simulated perpendicular field $B_{z}$, unit T, for all magnet sizes and orientations. Source:[3]

\subsubsection{Open-Circuit Voltage}

The $V_{o c} / f$ plots in figure 4.5 show that the $\mathrm{FP}$ employing the $3 \mathrm{~mm}$ magnet generated the lowest $V_{o c}$. This is expected because it has the lowest magnetic field strength. The EMF generated beneath the magnets when the magnet is located at the centre of the stator is approximately equal to [3]: 


$$
E M F \approx \int_{0}^{l} B v_{x} d y
$$

where $B$ is the magnetic field perpendicular to the HTS stator, $v_{x}$ is the magnet speed in the $\mathrm{x}$ direction and $l$ is the length of the magnet in the $\mathrm{y}$ direction. Compare with the 6 $\mathrm{mm}$ and $12 \mathrm{~mm}$ magnets equation 4.1 implies that the EMF induced by the $3 \mathrm{~mm}$ magnet was the lowest due to its weaker field strength. In chapter 2, it was shown that the non-zero average voltage produced by a HTS stator is proportional to the induced EMF (see equation 2.3). Since the the $3 \mathrm{~mm}$ induced the lowest EMF, the FP employing this magnet produced the lowest output voltage.

It is interesting to note the $V_{o c} / f$ for the $6 \mathrm{~mm}$ and $12 \mathrm{~mm}$ magnets are comparable (within the uncertainty in the stator misalignment), even though the magnetic field simulation shows that the $12 \mathrm{~mm}$ magnet perpendicular field strength is stronger. In chapter 2 the origin of the non-zero DC voltage generation in a HTS stator was discussed. It was pointed out that as the magnet passes over the stator eddy-currents are generated around the high magnetic field region in the superconducting material. The eddy-currents temporarily short-circuit the induced EMF and partially rectify the AC waveform. As a result, the average voltage generated over a complete cycle is not equal to zero [5]. The width of the $6 \mathrm{~mm}$ magnet is about half of the width of the HTS stator and this allows for a voltage generation for almost the entire period during which the magnet was crossing over the stator [3]. However, the $12 \mathrm{~mm}$ magnet is approximately the same width as the stator. With large magnet width the eddy-currents were produced in the HTS stator only when magnet was entering and leaving the stator. As a result, the final voltage integrated over a full rotor cycle for the $12 \mathrm{~mm}$ magnet turned out to be very close to the $6 \mathrm{~mm}$ magnet. This effect can be seen clearly in the $\Delta V / f$ waveforms for the $6 \mathrm{~mm}$ and $12 \mathrm{~mm}$ magnets shown in figure 4.8 . 


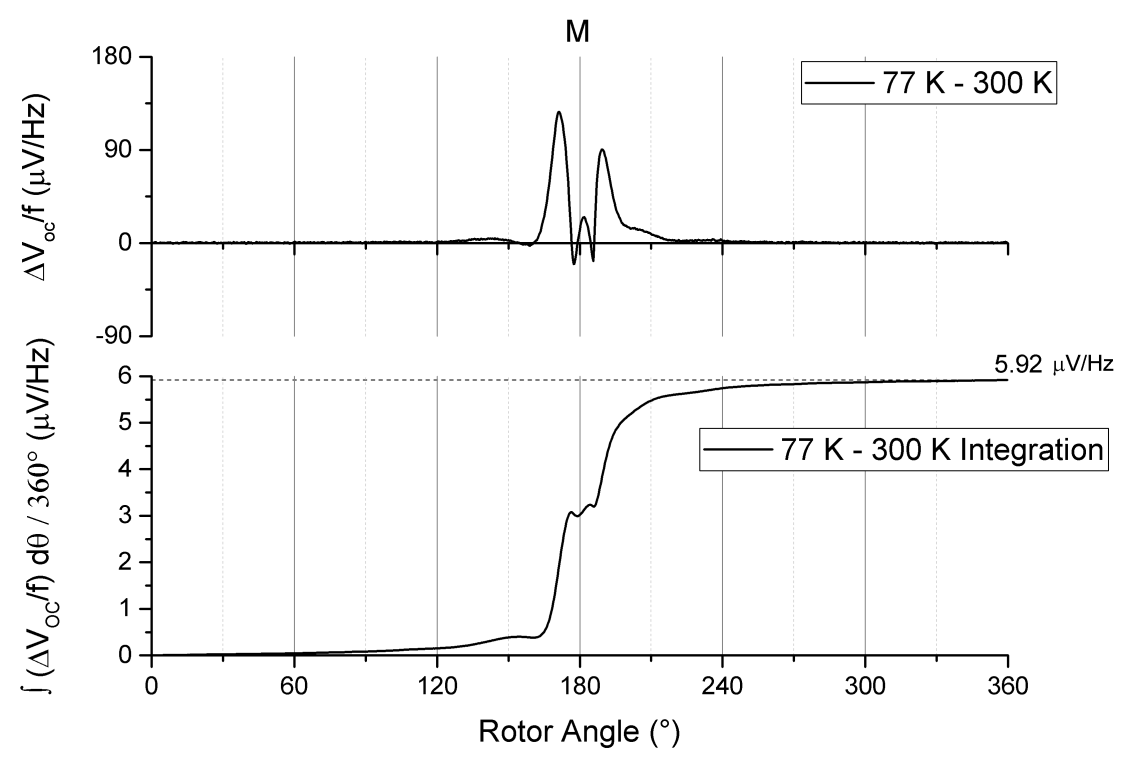

(a) $6 \mathrm{~mm}$ magnet

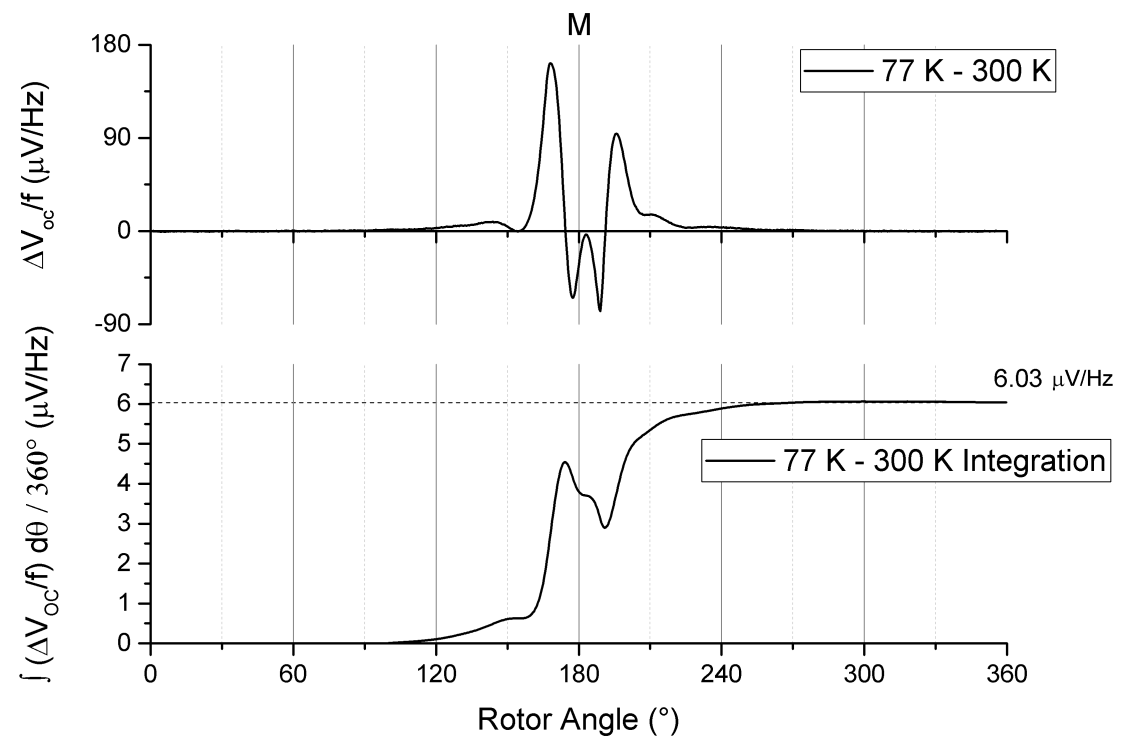

(b) $12 \mathrm{~mm}$ magnet

Figure 4.8: $\Delta V / f$ waveforms of Gen 1 system using $6 \mathrm{~mm} \& 12 \mathrm{~mm}$ magnets at vertical orientation, reverse direction, $3 \mathrm{~Hz}$. The final value of the integration for both magnet sizes are almost the same.

As pointed out earlier the $V_{o c}$ vs frequency data in figure 4.3 was not perfectly linear with respect to frequency. This also can be seen clearly in figures $4.5(\mathrm{a})$ and (b) where $V_{o c} / f$ decreased with frequency for all magnet sizes. This has been attributed to the screening effects of the induced eddy-currents circulating in the copper layer of the HTS tape [3, 14]. These eddy-currents are produced on the copper layer when the magnet is crossing the stator due to Lenz's Law. These eddy-currents induce magnetic fields, which work against the perpendicular magnetic field penetrating the superconducting wire. This effect is stronger as the eddy-currents increase in strength at higher frequency. Since the EMF in the superconductor is proportional to $B$ (equation 4.1), a reduction in the field strength in 
the superconductor material results in lower output voltage.

\subsubsection{Dynamic Resistance}

Figures $4.5 \mathrm{c}$ and $4.5 \mathrm{~d}$ show that the dynamic resistance in the stator increases with magnet size. In section 2.4 the dynamic resistance of a HTS FP was discussed. It was shown that $R_{d}$ is proportional to $B$ and the interaction length of the magnetic field with the stator [19]. Since the large magnets had stronger field strength, they produced higher $R_{d}$.

Similar to the $V_{o c} / f$, the normalised dynamic resistance $R_{d} / f$ also decreases with frequency for all magnet sizes due to the eddy-current screening effect.

\subsubsection{Short-Circuit Current}

As mentioned in chapter $2, I_{s c}$ is a derived quantity $I_{s c}=V_{o c} / R_{d}$. It is the maximum current that can be supplied by the FP when the load resistance is zero. The results in figures 4.5e and $4.5 \mathrm{f}$ show that $I_{s c}$ is not affected by the eddy-current screening effect. The reason is the changes in $V_{o c}$ compensates for the changes in $R_{d}$. The FP with $12 \mathrm{~mm}$ magnet produced the smallest $I_{s c}$ because the $R_{d}$ was large.

\subsubsection{Angled Orientation}

Figure 4.9 shows the results of the angled orientation for all magnet sizes. The high frequency data (17 and $25 \mathrm{~Hz}$ ) for the $12 \mathrm{~mm}$ magnet is not available for comparison. 


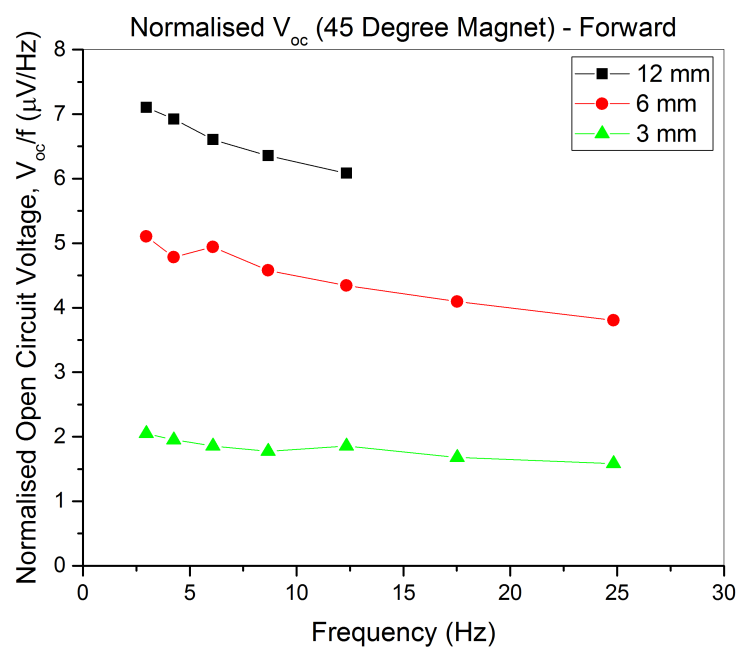

(a) $V_{o c} / f$, forward

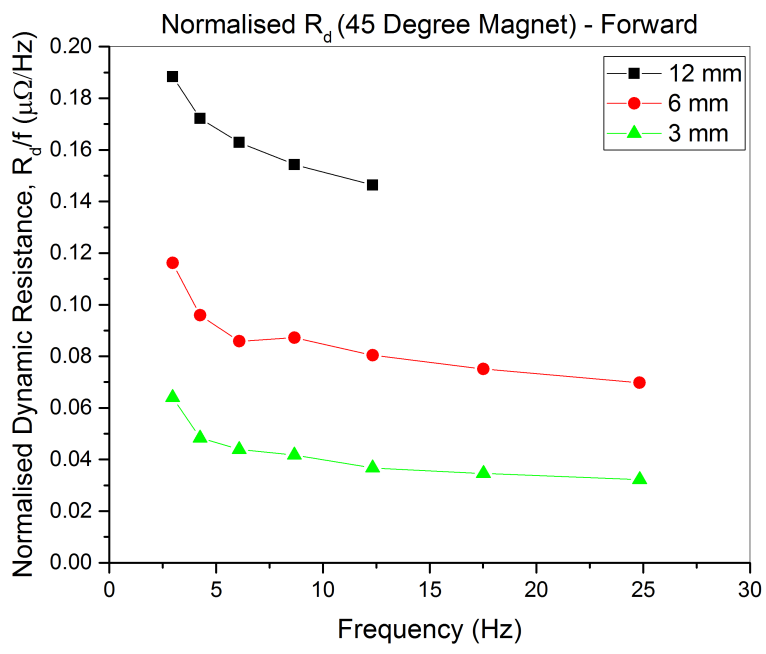

(c) $R_{d} / f$, forward

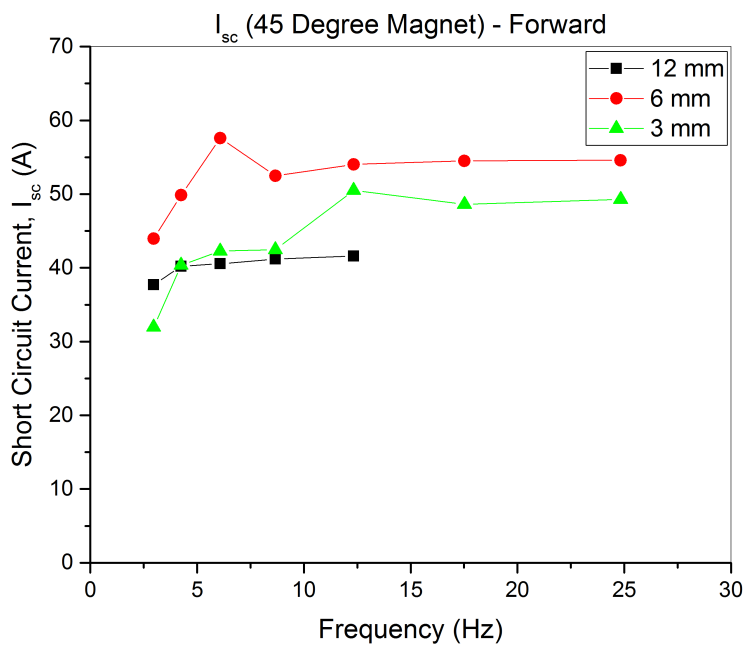

(e) $I_{s c}$, forward

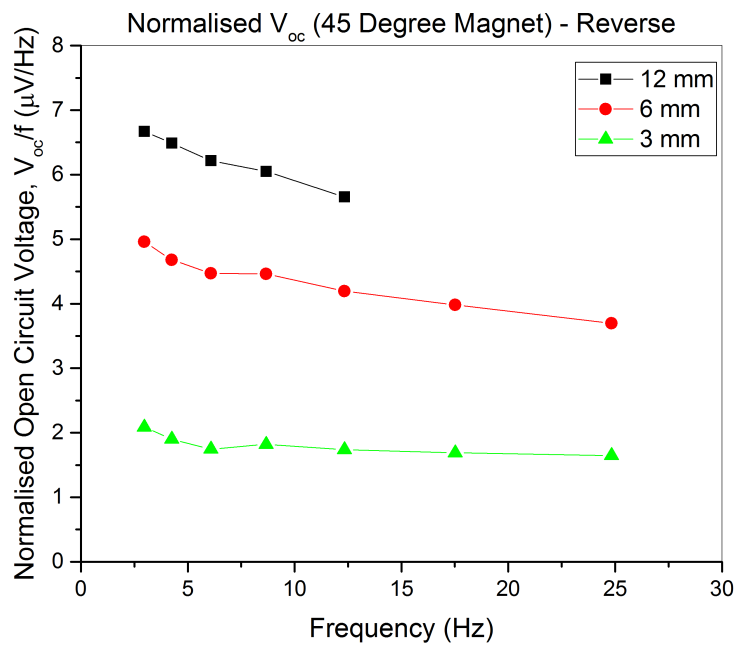

(b) $V_{o c} / f$, reverse

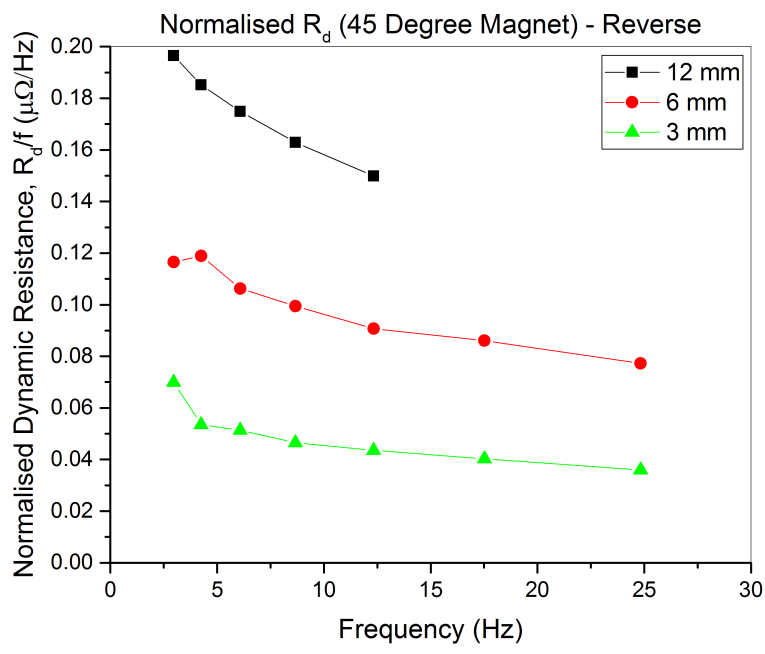

(d) $R_{d} / f$, reverse

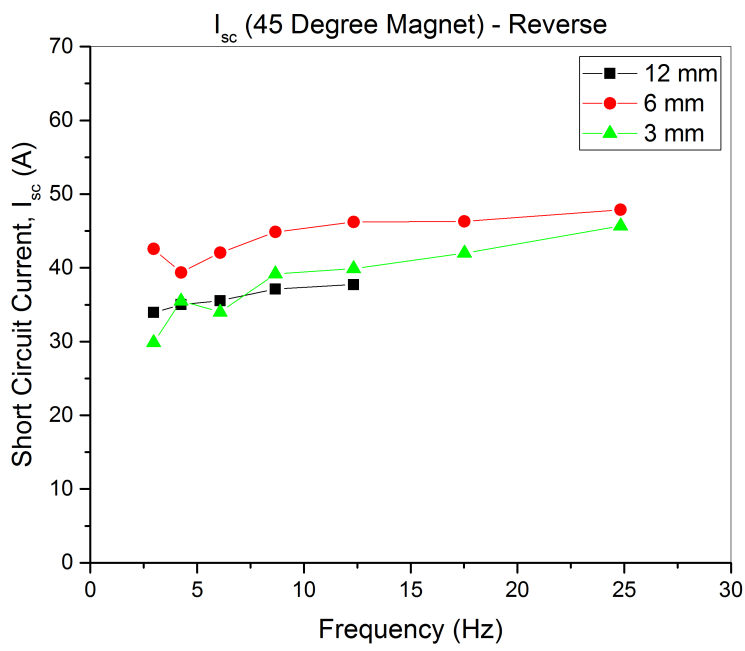

(f) $I_{s c}$, reverse

Figure 4.9: Gen $1 \mathrm{FP}$ performance using $3 \mathrm{~mm}, 6 \mathrm{~mm} \& 12 \mathrm{~mm}$ magnets at angled orientation. The lines joining the data points are guides to the eye. The forward data has been published in [3] and the reverse data is included for comparison.

Figure 4.10 shows the simulated magnetic field profile at the stator for each of the magnet 
sizes. The simulated perpendicular field $B_{z}$ was shown in figure 4.7.

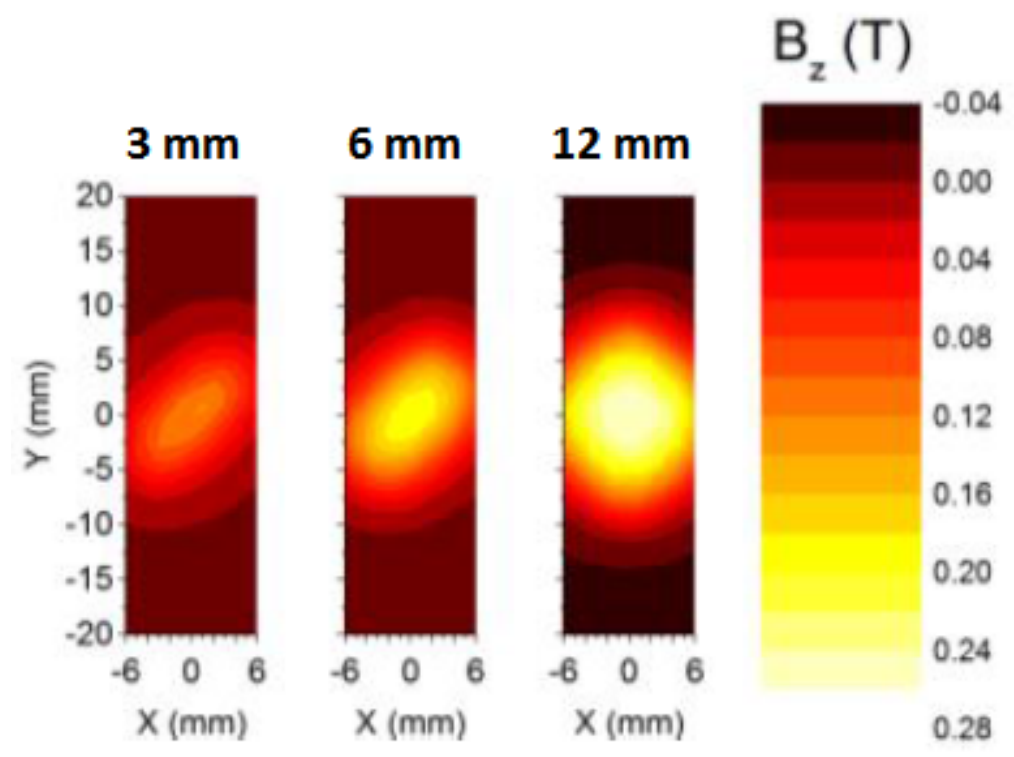

Figure 4.10: Simulated stator magnetic field profile, angled orientation. The parameter $\mathrm{x}$ is the length measured along the width of the stator. The parameter $y$ is the length measured along the length of the stator. Flux gap $3.7 \mathrm{~mm}$. Source:[3]

\subsubsection{Open-Circuit Voltage}

The $12 \mathrm{~mm}$ magnet had the strongest magnetic field strength and the longest length measured in the y direction. From equation 4.1, this implies that the FP employing the $12 \mathrm{~mm}$ magnet produced the highest $V_{o c}$. The data for all magnet sizes show the eddy-current shielding effect.

\subsubsection{Dynamic Resistance}

The data for all magnet sizes show the eddy-current shielding effect. The $12 \mathrm{~mm}$ magnet produced the largest $R_{d}$ compared with other magnet sizes because:

1. It had the strongest magnetic field strength.

2. It had the longest effective interaction length of the magnetic field with the stator.

\subsubsection{Short-Circuit Current}

The $I_{s c}$ at $45^{\circ}$ orientation for all magnet sizes is independent of frequency. It is not affected by the eddy-current screening effect because the changes in $V_{o c}$ compensates for the changes in $R_{d}$. 


\subsubsection{Horizontal Orientation}

Figure 4.11 shows the results of the horizontal orientation for all magnet sizes. The $12 \mathrm{~mm}$ magnet data is identical to the vertical orientation. The high frequency data (17 and $25 \mathrm{~Hz})$ for the $12 \mathrm{~mm}$ magnet is not available for comparison. 


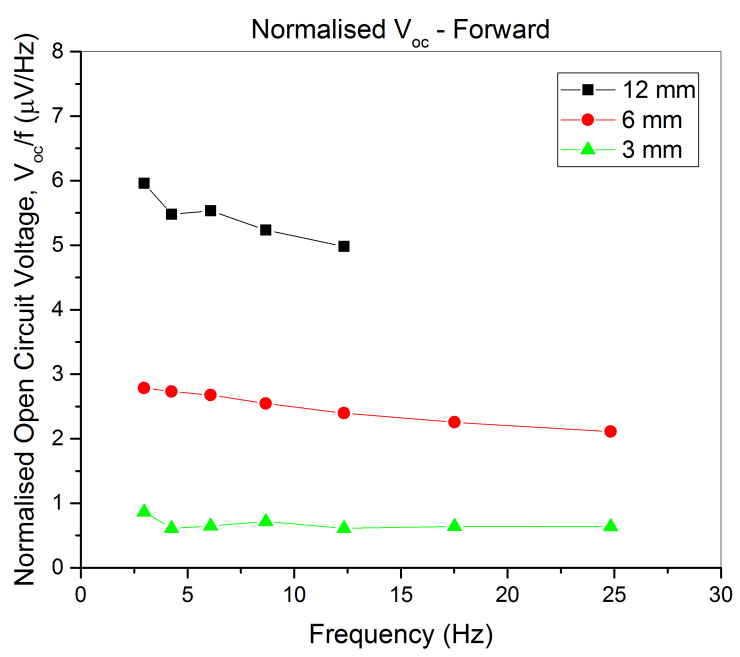

(a) $V_{o c} / f$, forward

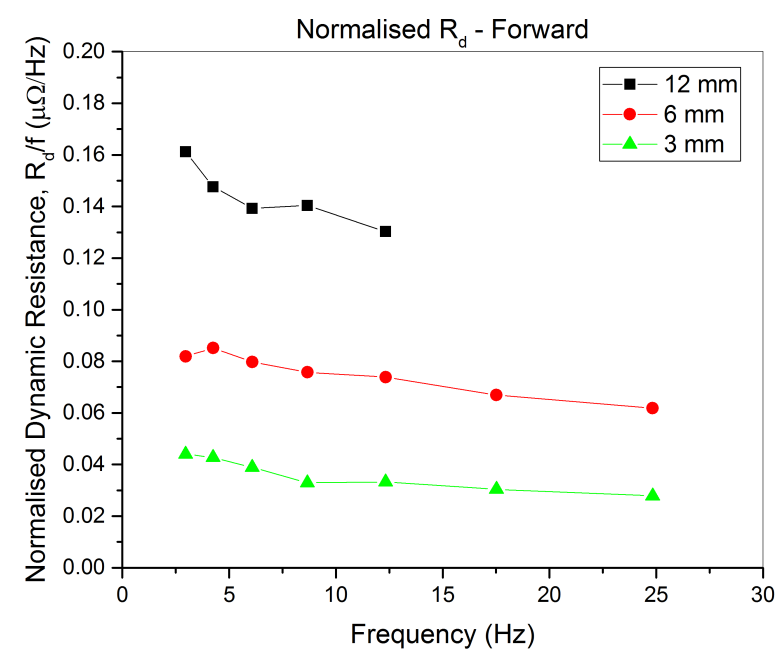

(c) $R_{d} / f$, forward

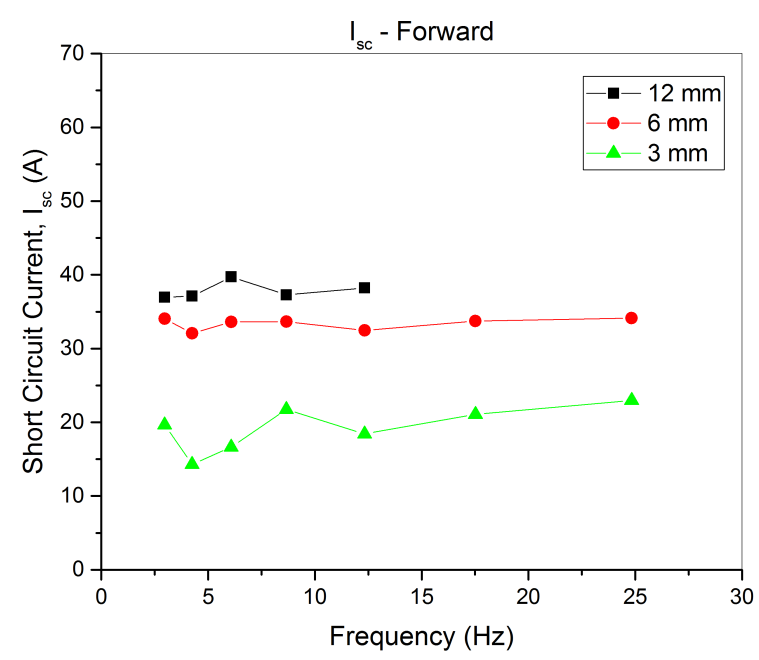

(e) $I_{s c}$, forward

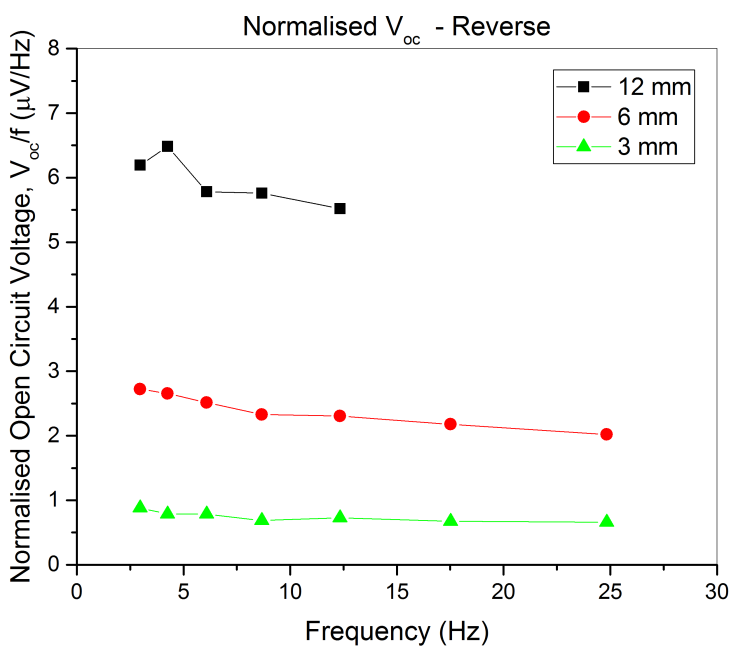

(b) $V_{o c} / f$, reverse

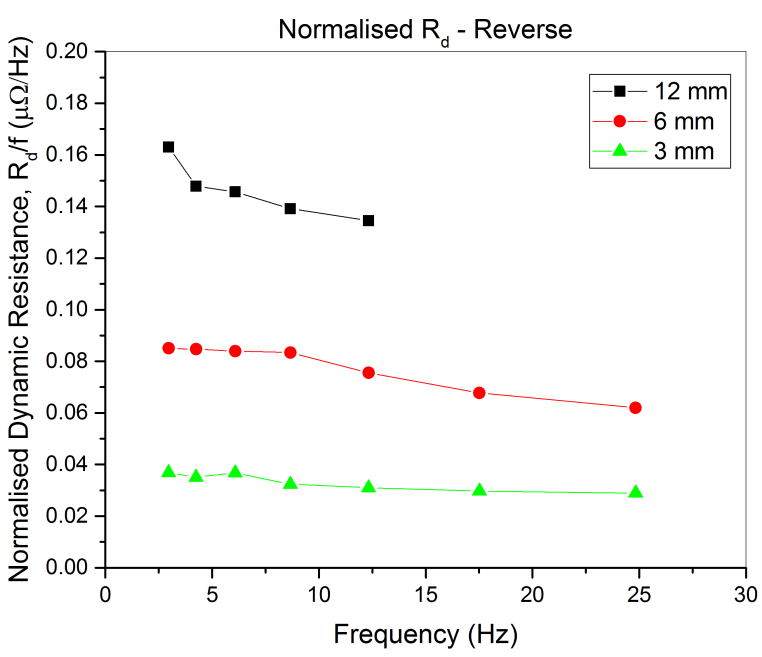

(d) $R_{d} / f$, reverse

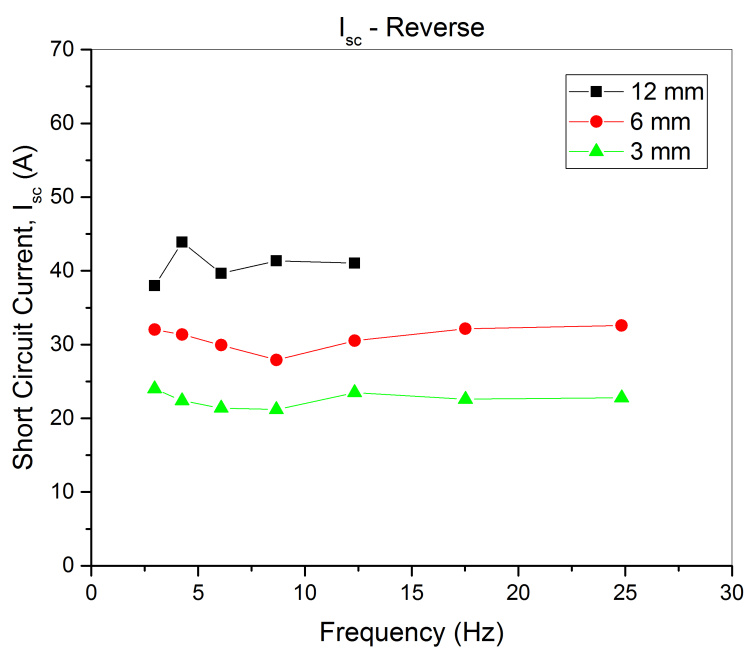

(f) $I_{s c}$, reverse

Figure 4.11: Gen $1 \mathrm{FP}$ performance using $3 \mathrm{~mm}, 6 \mathrm{~mm} \& 12 \mathrm{~mm}$ magnets at horizontal orientation. The lines joining the data points are guides to the eye. The forward data has been published in [3] and the reverse data is included for comparison.

Figure 4.12 shows the simulated magnetic field at the stator for each of the magnet sizes. 
The simulated perpendicular field $B_{z}$ was shown in figure 4.7.

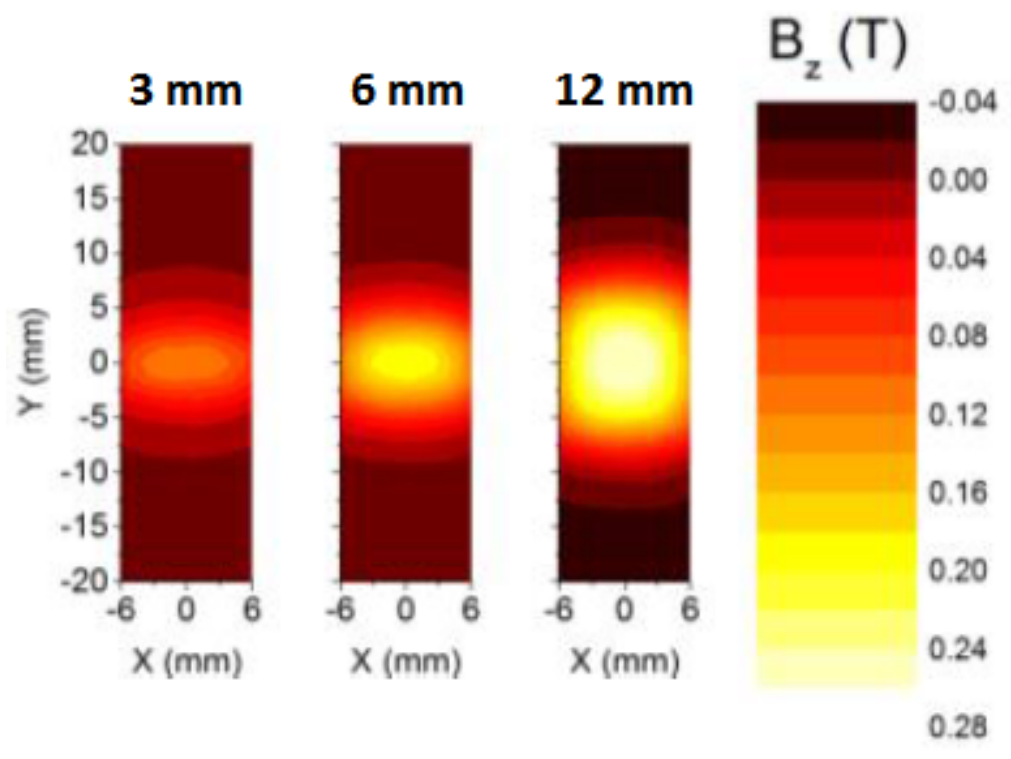

Figure 4.12: Simulated stator magnetic field profile, horizontal orientation. The parameter $\mathrm{x}$ is the length measured along the width of the stator. The parameter $y$ is the length measured along the length of the stator. Flux gap $3.7 \mathrm{~mm}$. Source:[3]

\subsubsection{Open-Circuit Voltage}

The $12 \mathrm{~mm}$ magnet produced the largest $V_{o c}$ because, in addition to the strongest magnetic field strength, it also had the longest length in the y direction (see equation 4.1) .

\subsubsection{Dynamic Resistance}

The $12 \mathrm{~mm}$ magnet produced the largest $R_{d}$ because it had the strongest magnetic field strength and the longest interaction length of the magnetic field with the stator.

\subsubsection{Short-Circuit Current}

The $I_{s c}$ is almost constant across the frequency range. It is not affected by the eddy-current screening effect because the changes in $V_{o c}$ compensates for the changes in $R_{d}$.

Finally, it is useful to compare the results produced using a fixed magnet size for different orientations. This is discussed in the next two sections.

\subsubsection{Fixed Magnet Size (3 $\mathrm{mm} \& 6 \mathrm{~mm}$ Magnets)}

Figure 4.13 shows the plots for vertical, angled and horizontal orientations for the $3 \mathrm{~mm}$ magnet. Figure 4.14 shows the plots for vertical, angled and horizontal orientations for the $6 \mathrm{~mm}$ magnet. 

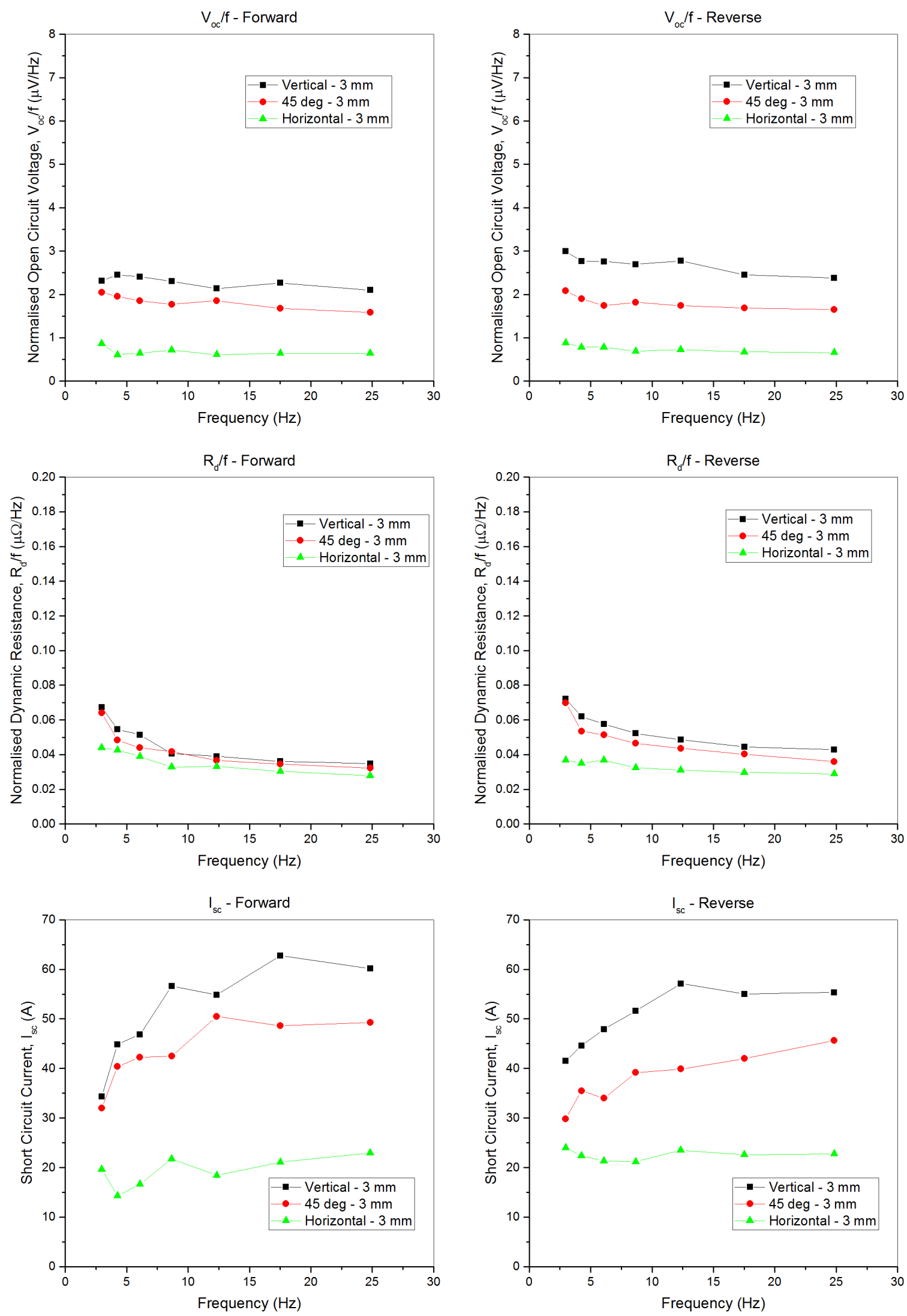

Figure 4.13: Gen 1 FP performance using $3 \mathrm{~mm}$ magnets for all magnet orientations. The lines joining the data points are guides to the eye. The forward data has been published in [3] and the reverse data is included for comparison. 

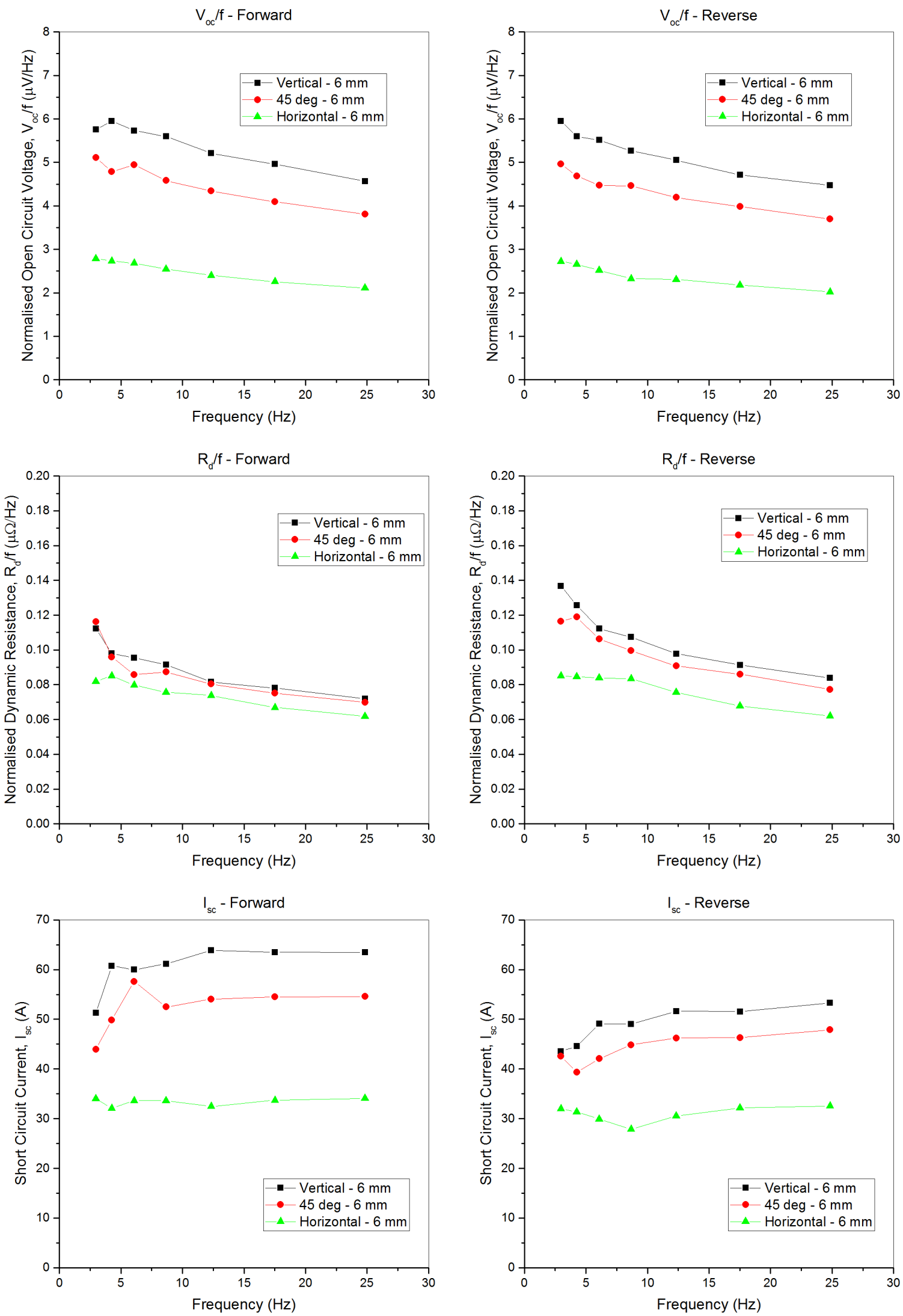

Figure 4.14: Gen $1 \mathrm{FP}$ performance using $6 \mathrm{~mm}$ magnets for all magnet orientations. The lines joining the data points are guides to the eye. The forward data has been published in [3] and the reverse data is included for comparison.

It can be seen in both figures that the vertically oriented magnets produced the highest $V_{o c}$. This reason is that the vertical magnets have smaller width in the $\mathrm{x}$ direction. For this 
reason, the eddy-currents short circuit the EMF and the stators generate voltage for almost the entire period during which the magnets are crossing over the stators [3]. Another reason for the high $V_{o c}$ is that the vertically oriented magnets have the longest length measured in the $\mathrm{y}$ direction.

The $R_{d}$ for all three magnet orientations are very similar. The reason is that $R_{d}$ is also determined by the total flux passing the stator per rotation cycle [3]. Since the magnetic field strengths are the same for all orientations, the amount of flux passing the stator is the same and the $R_{d}$ for all magnet orientations are almost identical.

\subsubsection{Fixed Magnet Size (12 mm Magnet)}

Figure 4.15 shows the results for the $12 \mathrm{~mm}$ magnets at vertical (also equal to horizontal) and angled orientations. The length of the angled magnet measured in the y direction is longer than the vertical magnet. For this reason it produced larger EMF (see equation 4.1) and therefore higher output voltage. The angled magnet also produced larger $R_{d}$ compared with the vertical magnet because of the longer interaction length between the magnetic field and the stator. 

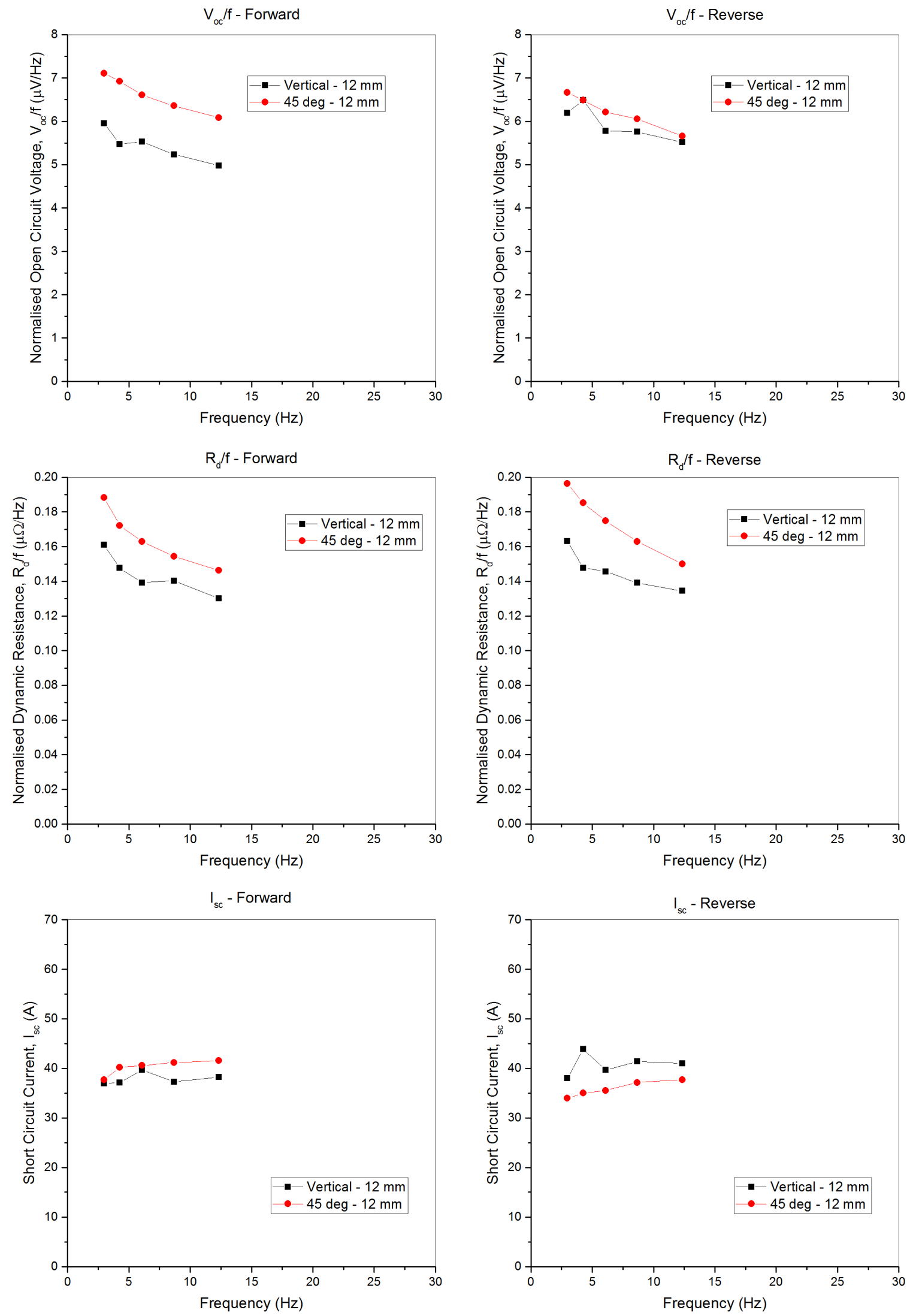

Figure 4.15: Gen $1 \mathrm{FP}$ performance using $12 \mathrm{~mm}$ magnet for all magnet orientations. The lines joining the data points are guides to the eye. The forward data has been published in [3] and the reverse data is included for comparison. 


\subsubsection{Summary}

The observation on the experimental data can be summarised as follows:

1. The dynamic resistance $R_{d}$ of the FP increased with the magnetic field strength of the permanent magnet.

2. The $R_{d}$ increased with the interaction length between the magnetic field and the stator.

3. If the interaction lengths were comparable, the magnet orientations did not have too much effect on the $R_{d}$. The reason was that the $R_{d}$ was determined by the total flux passing across the stator.

4. The $V_{o c}$ increased with the magnet length measured in the y direction.

5. For equal magnetic field strength, the magnets with the width (measure in the $\mathrm{x}$ direction) narrower than the stators produced higher $V_{o c}$.

6. The $I_{s c}$ of the FP is derived from $V_{o c}$ and the $R_{d}$. It was not affected by the eddycurrent screening effect because the changes in $V_{o c}$ due to this effect compensated for the changes in $R_{d}$.

For an optimal FP performance, the "design rules" [3] are:

1. For higher $V_{o c}$, the length of the magnet in the x direction should be smaller than the stator width.

2. For higher $V_{o c}$, the length of the magnet in the y direction should be longer. However, since $R_{d}$ also increased with the length, it should only be increased until a balance is achieved between $V_{o c}$ and $R_{d}$. 


\subsection{Gen 2 Flux Pump System Experimental Results}

The second generation (Gen 2) FP system has been used in this project to investigate the frequency dependent behaviour of the HTS dynamo type FP and the effect of the stator width on the performance of the FP. Three types of stators were used in the experiments. In the first part of this section, the materials, the construction and the installation of the stators are discussed. Then, the $\mathrm{AC}$ waveforms are studied in order to understand the mechanism that is responsible for the DC voltage output of the FP. Contact resistances occur in the FP circuit and the method employed in the experiment to determine the total value is shown. After that, the DC operation of the FP and the performance are studied and the frequency dependent behaviour is explored. This is then followed by the study of the AC waveforms measured at different FP operating frequencies. Finally, the observation on the bi-stable thermal mode of the FP are discussed.

For this project the existing Gen 2 experimental setup was used to run the experiments. However, new FP stators were made from superconductor tapes and installed onto the system for the work mentioned here. Some of the results and discussions presented in this section have been published in IEEE Transactions on Applied Superconductivity [7].

\subsubsection{Experiment Samples Preparation}

The stators employed in the experiments were made from AMSC $46 \mathrm{~mm}$ wide Ag-coated tape. This led to stators which were much wider than the $6 \mathrm{~mm} \times 12 \mathrm{~mm} \mathrm{Nd-Fe-B}$ rotor permanent magnets. The flux gap, measured from the surface of the stator to the surface of the magnets, was $7.5 \mathrm{~mm}$. The $I_{c}$ of the tape was about $225 \mathrm{~A} / \mathrm{cm}$, measured at $77 \mathrm{~K}$ at 1 $\mu \mathrm{V} / \mathrm{cm}$ criterion. Three stators were prepared for the experiments. The first stator (Stator I) had a full $46 \mathrm{~mm}$ width. The second stator (Stator II) was slit with a sharp knife to form two parallel stators, each with a width of approximately $23 \mathrm{~mm}$. The third stator (Stator III) was slit into three parallel stators, each with a width of approximately $15 \mathrm{~mm}$. The length of the original tapes was about $230 \mathrm{~mm}$ and the slit length was about $140 \mathrm{~mm}$ so that the ends of the stators II and III were still electrically (superconducting) connected. Two copper voltage taps were soldered onto the stators for voltage measurement. The schematic of the stators are shown in figure 4.16 and photos of the stators mounted on the iron yoke are shown in figure 4.17 . 


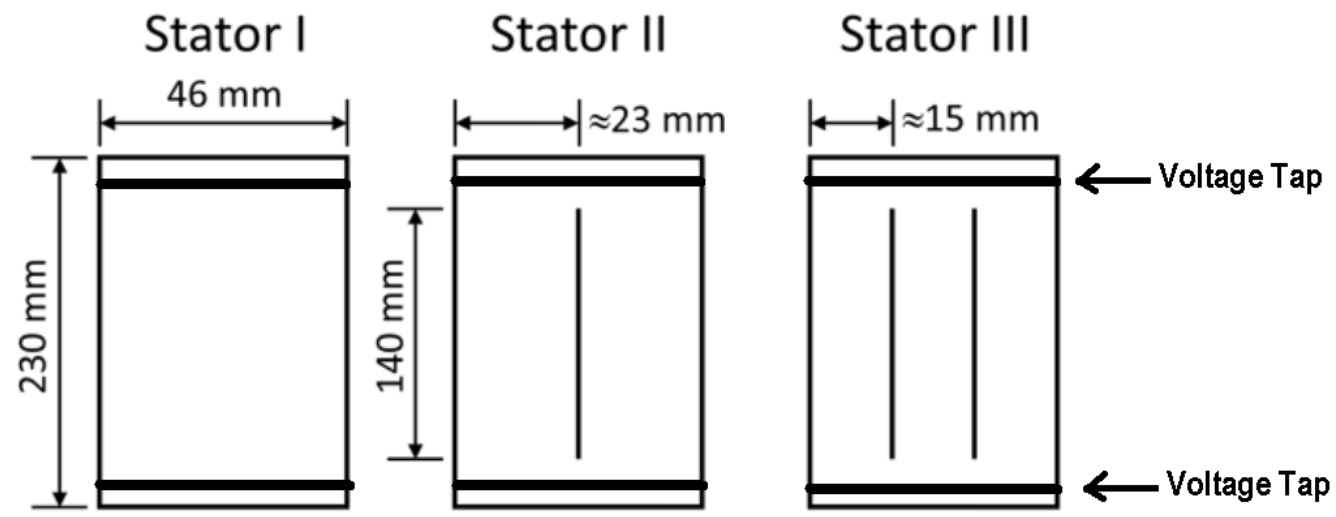

Figure 4.16: Slit tapes. Published in:[7].

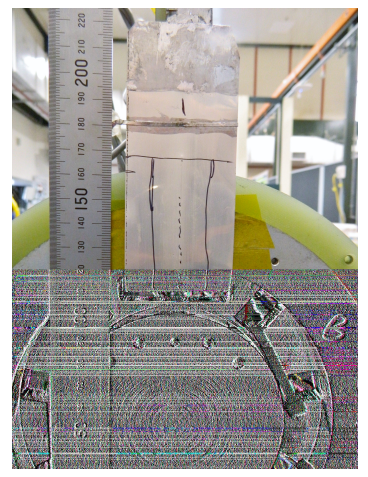

(a) Stator I

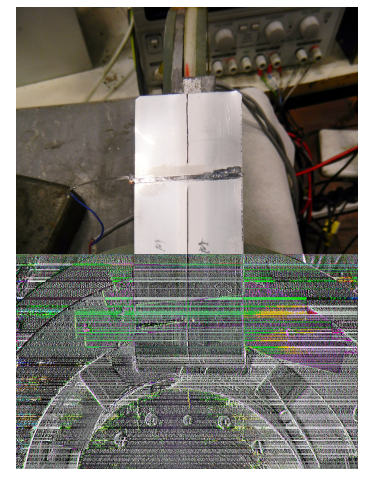

(b) Stator II

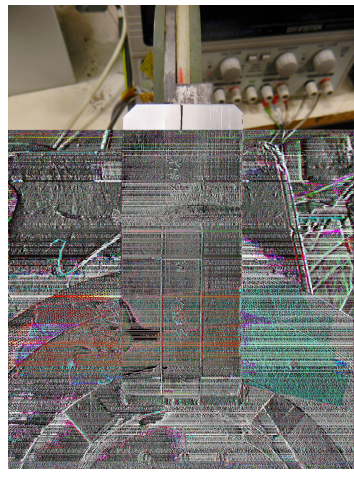

(c) Stator III

Figure 4.17: Stators installed on the FP system.

\subsubsection{The AC Waveforms and the Open-Circuit Voltage Generation}

As discussed in section 2, a HTS dynamo type FP is capable of producing a DC voltage output. It was shown that the integral of the voltage measured across the stator in superconducting state over a full rotor cycle is not zero. Figure 4.18a shows an example of the $\mathrm{AC}$ voltages measured across stator III over a full rotor cycle $\left(360^{\circ}\right)$ at $27 \mathrm{~Hz}$. These voltage waveforms are broadly similar to those observed for the Gen 1 FP system. The letter M denotes the moment when a rotor magnet was located at the centre of the stator. The solid line is the $\mathrm{AC}$ voltage measured at $77 \mathrm{~K}$ in liquid $\mathrm{N}_{2}$ when the stator was in the superconducting state. The orange dashed line is the voltage measured at room temperature when the stator was in the normal conducting state. The red dotted line shows the average DC voltage which was measured separately using a low-pass filter. It can be seen clearly that the shapes of the $77 \mathrm{~K}$ and $300 \mathrm{~K} \mathrm{AC}$ waveforms are very different. Figure 4.18b shows the integration of the waveforms over a full rotor cycle (9 magnets passing over). For the $77 \mathrm{~K}$ waveform the final integral value is about $0.01 \mathrm{mV} / \mathrm{Hz}(0.29 \mathrm{mV})$, whereas for the $300 \mathrm{~K}$ waveform the result is zero. 


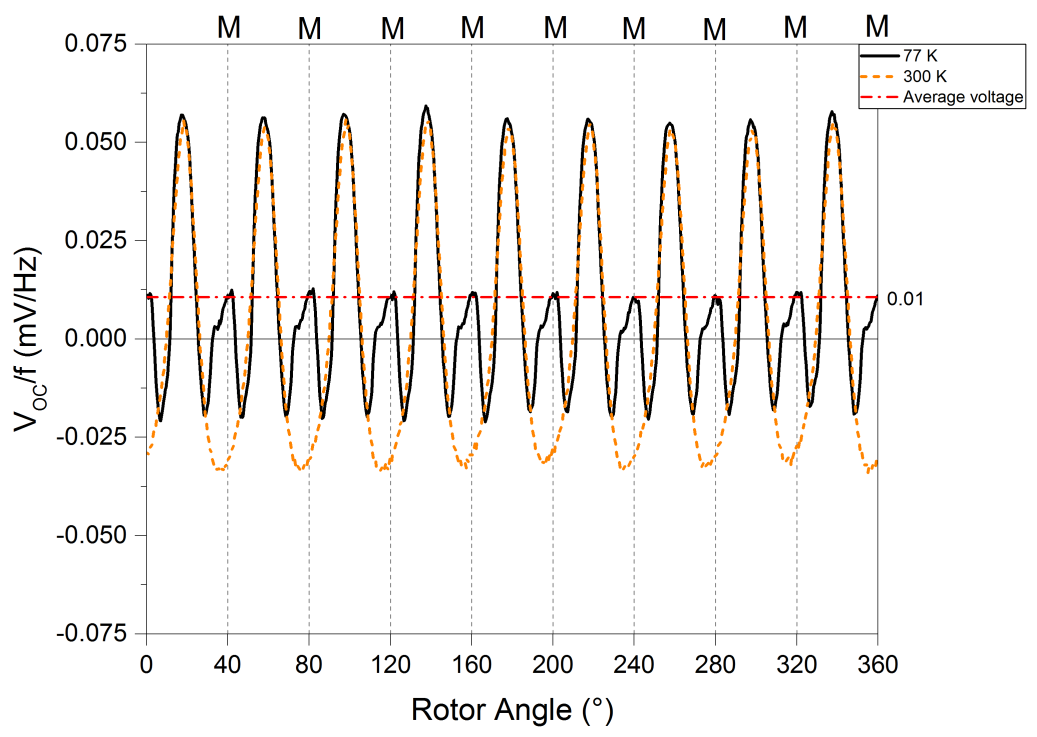

(a) AC waveforms measured at $77 \mathrm{~K}$ and $300 \mathrm{~K}$.

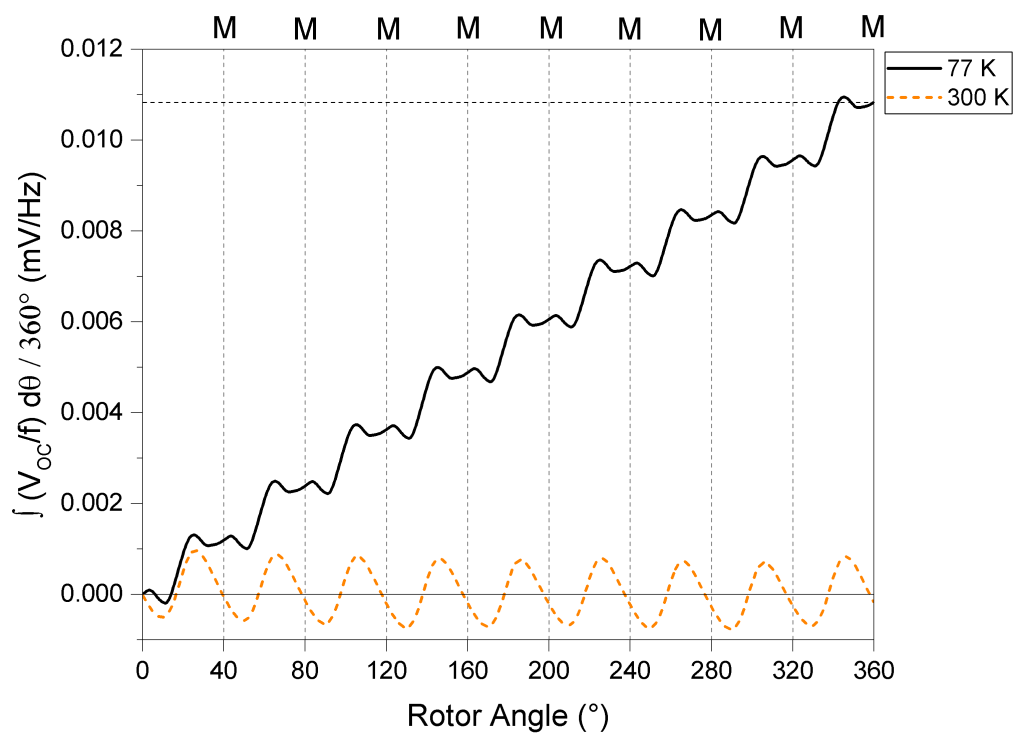

(b) AC waveform integration over a full rotor cycle.

Figure 4.18: Stator III frequency-normalised open-circuit voltage waveform measured at 27 $\mathrm{Hz}$.

\subsubsection{Contact Resistance}

The total contact resistance in the FP circuit exists at the connections between the FP stator and the copper connectors, and between the stator and the current coil (see figure $3.4 \mathrm{~b})$. Since the inductance of the current coil is a known value $(1.97 \mathrm{mH})$, the contact resistance $R_{c}$ can be inferred from the rate of decay of the current in the circuit. If the FP is turned off when the current in the circuit has reached $I_{1}$, the current decays due to the power dissipation in the contact resistances. The decaying current is described by: 


$$
I_{\text {decay }}(t)=I_{1} e^{-R_{c} t / L}
$$

and in the linear form:

$$
\ln \left(I_{\text {decay }}\right)=\ln \left(I_{1}\right)-\frac{R_{c}}{L} t
$$

The total value of the contact resistances $R_{c}$ can be obtained by fitting equation 4.3 to the current decay data. An example of the decay current and the curve fit is shown in figure 4.19. The non-linearity of the curve observed from time 0 seconds to about 375 seconds is believed to be due to the decay of the screening current in the coating layer of the current coil. Therefore the data within this period is not included in the curve fit. The value of the total contact resistances obtained from the fit is $0.60 \mu \Omega$. In general, the value of the contact resistances in the experiments was $<1 \mu \Omega$.

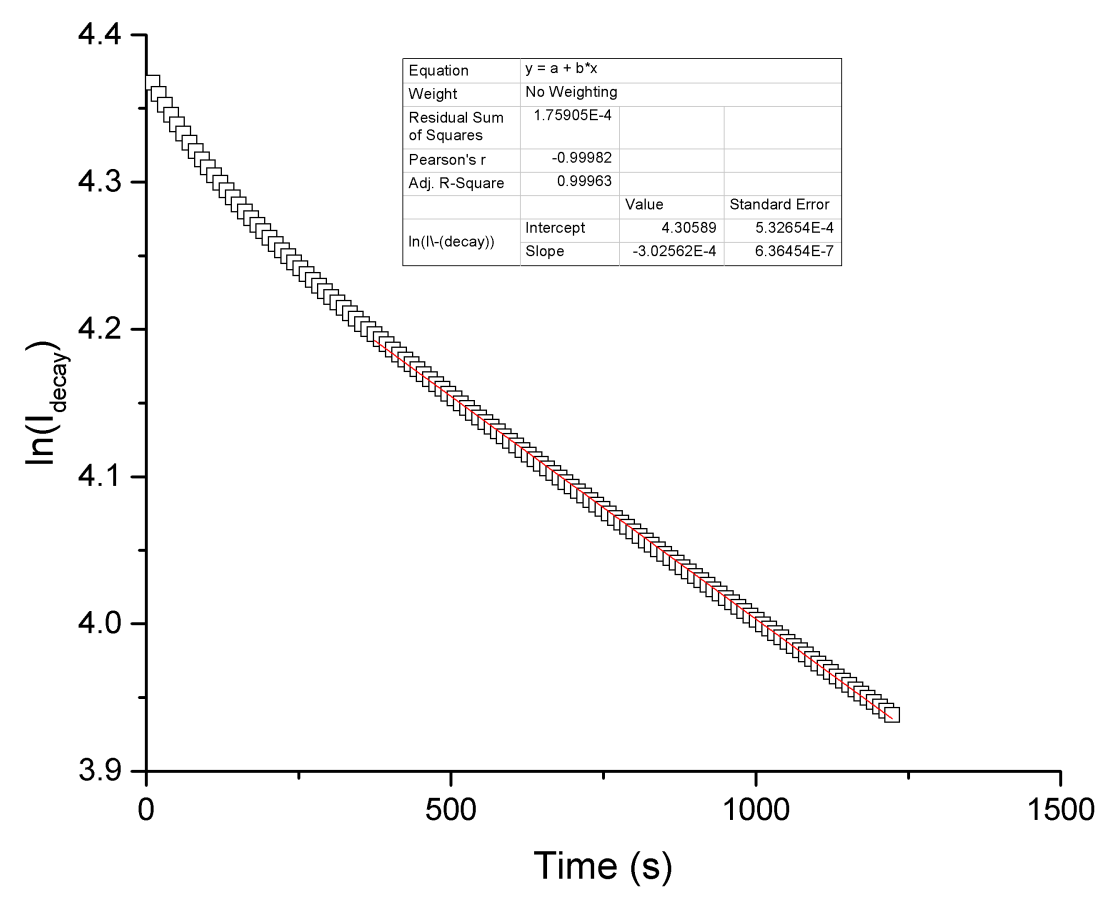

Figure 4.19: Stator III decay current curve fit.

\subsubsection{The Performance and the Frequency Dependent Behaviour of the FP}

The DC behaviour of the HTS dynamo-type FP and the model were discussed in section 2.4. The model is described by equation 2.5 and the parameters that characterise the performance of the FP are the open-circuit voltage $\left(V_{o c}\right)$, dynamic resistance $\left(R_{d}\right)$ and short-circuit current $\left(I_{s c}\right)$. These parameters are obtained from the $I-V$ curve in which the FP voltage ( $V_{F P}$, measured across the stator) is plotted with respect to the circuit current. The $I-V$ curves produced by stator III at various frequencies are shown in figure 4.20. 


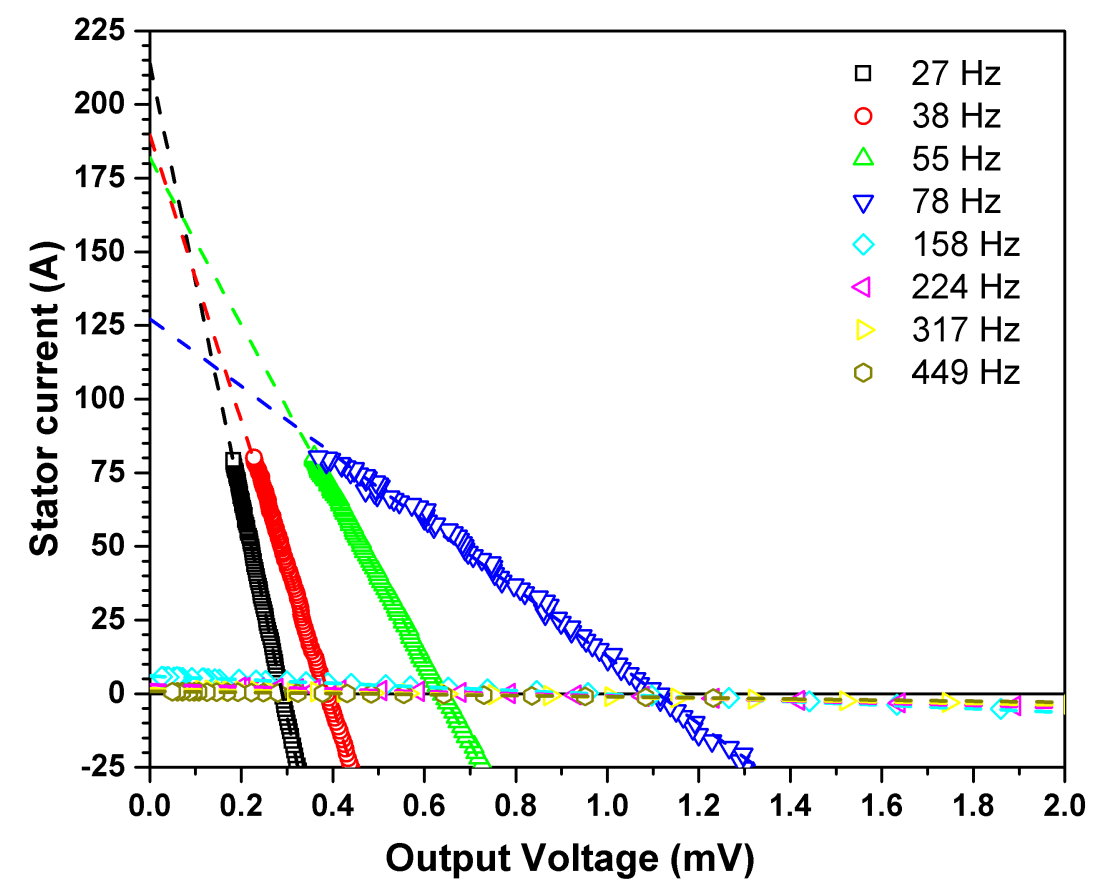

Figure 4.20: Stator III $I-V$ curve. The lines drawn are the linear line fits. Published in:[7].

Figure 4.21 depicts the DC current vs time plot of stator III operating at different frequencies. It shows that at low operating frequencies $(27-78 \mathrm{~Hz})$ the current in stator III increases linearly and reaches a maximum current $I_{0}$, which is limited by the total contact resistance and the current coil $I_{c} \approx 95$ A. However, at high operating frequencies $(\geq 158$ $\mathrm{Hz}$ ) the maximum current value is much lower. These two distinctive operating frequency regimes can be clearly seen in $I-V$ plots in figure 4.20. For example, at the operating frequencies from $27 \mathrm{~Hz}$ to $55 \mathrm{~Hz}$ the $\mathrm{FP}$ showed high $I_{s c}$ value. At $78 \mathrm{~Hz}$ the $I_{s c}$ value is lower due to the increase in the dynamic resistance (the inverse of the slope of the $I-V$ curve). At higher frequencies (158 Hz and above) the FP produces almost zero $I_{s c}$ due to large $R_{d}$. 


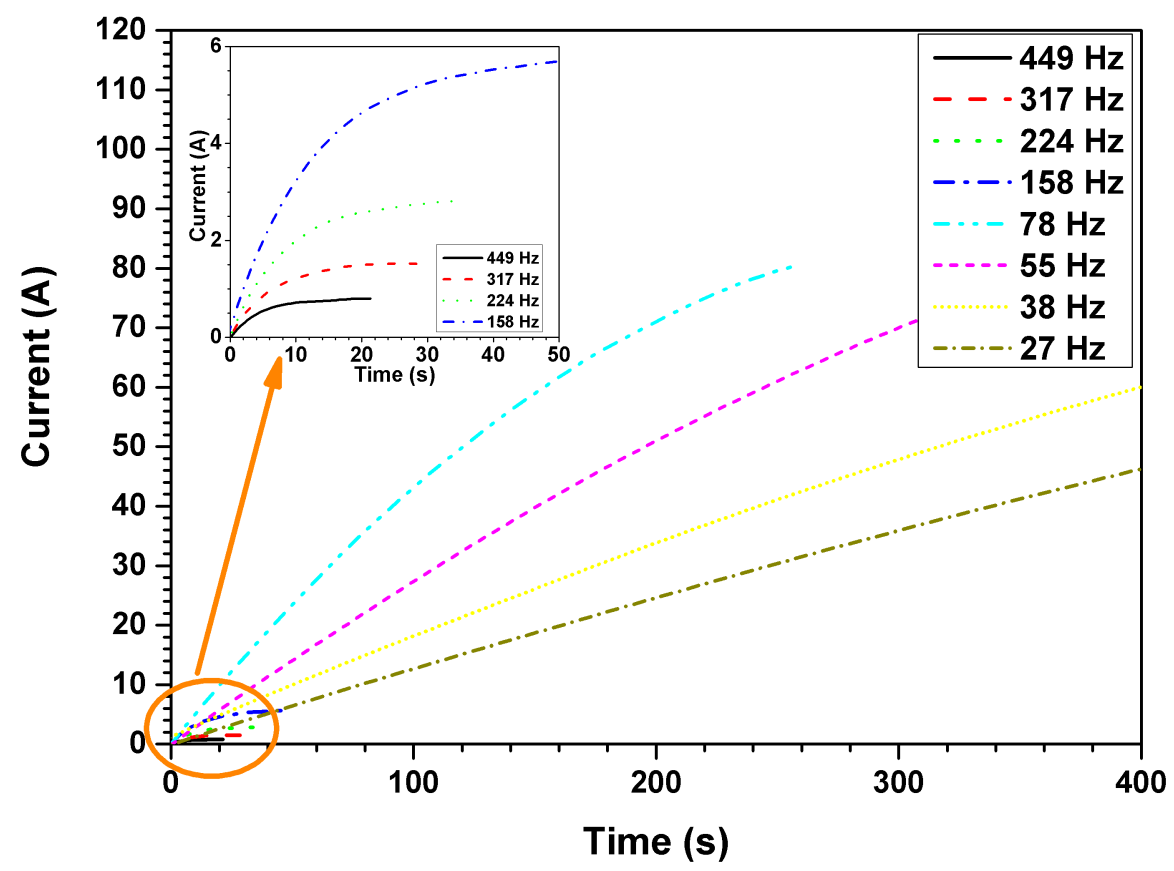

Figure 4.21: Stator III DC current vs time plot. Published in:[7].

Similar frequency dependent behaviour was also observed on the FPs employing stators I and II and their $I-V$ curves are shown in figures 4.22 and 4.23 respectively.

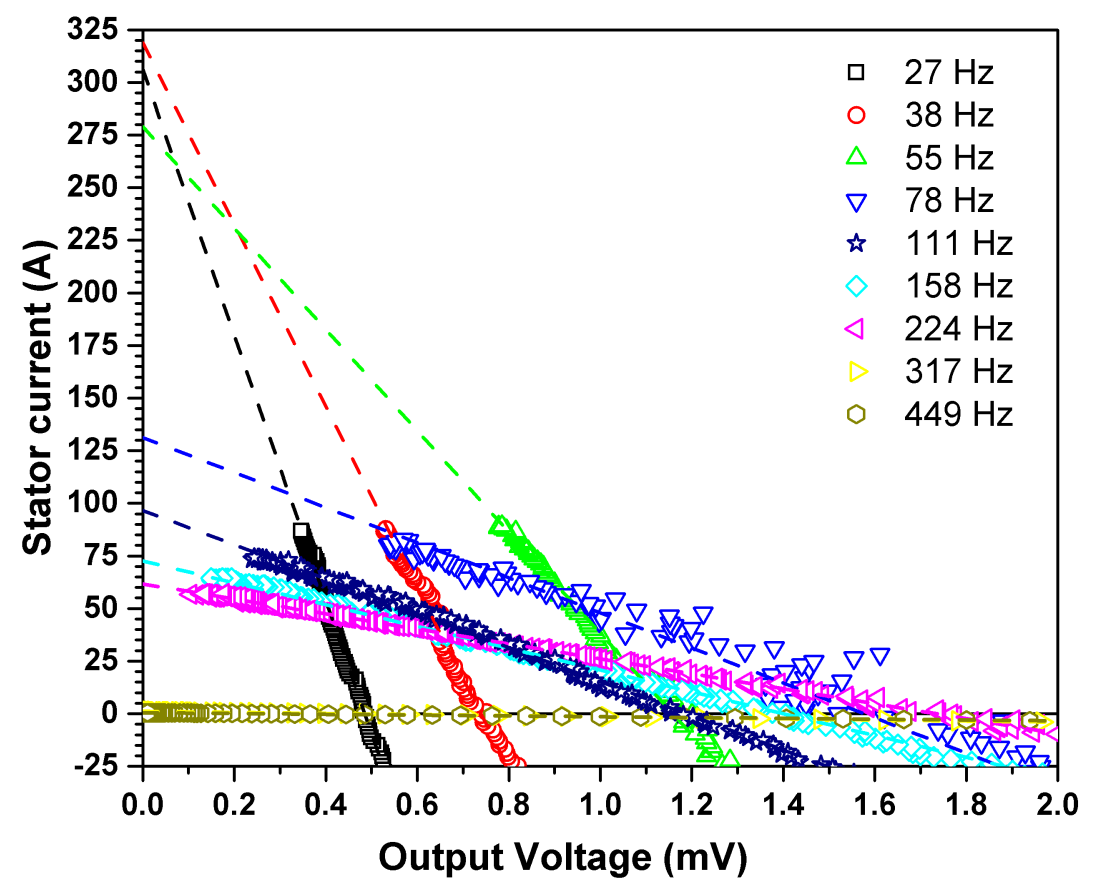

Figure 4.22: Stator I $I-V$ curve. The lines drawn are the linear line fits. Published in:[7]. 


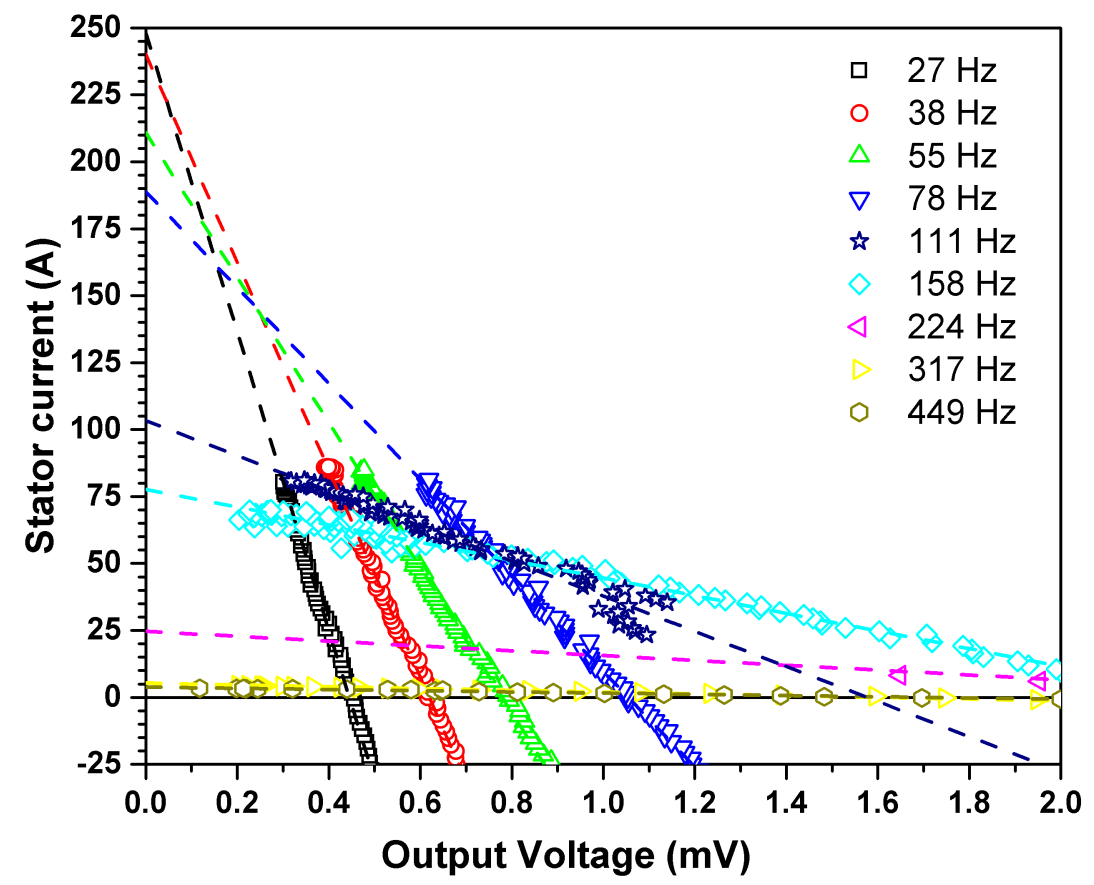

Figure 4.23: Stator II $I-V$ curve. The lines drawn are the linear line fits. For the frequency $224 \mathrm{~Hz}$ there are more data points beyond $2.0 \mathrm{mV}$, which are not shown in the plot but used in the line fits. Published in:[7].

In order to study the effect of the operating frequency on the performance, the parameters ( $V_{o c}, R_{d}$ and $I_{s c}$ ) extracted from the $I-V$ curves were plotted with respect to the frequency. Figure 4.24a shows the performance of stator I and the plots are divided into three operating regimes. In the low frequency regime, the FP voltage and $R_{d}$ increase linearly with frequency. In the mid frequency regime, the rate of increase of the dynamic resistance is higher and the FP voltage dropped. The combination of the changes in $V_{o c}$ and $R_{d}$ results in a drastic reduction in $I_{s c}$. In the high frequency regime, $V_{o c}$ drops drastically with respect to frequency, whereas $R_{d}$ increases rapidly. As a result, the FP is no longer able to produce a measurable output current. Stators II and III also showed similar frequency dependent behaviour (figures $4.24 \mathrm{~b}$ and $4.24 \mathrm{c})$. 


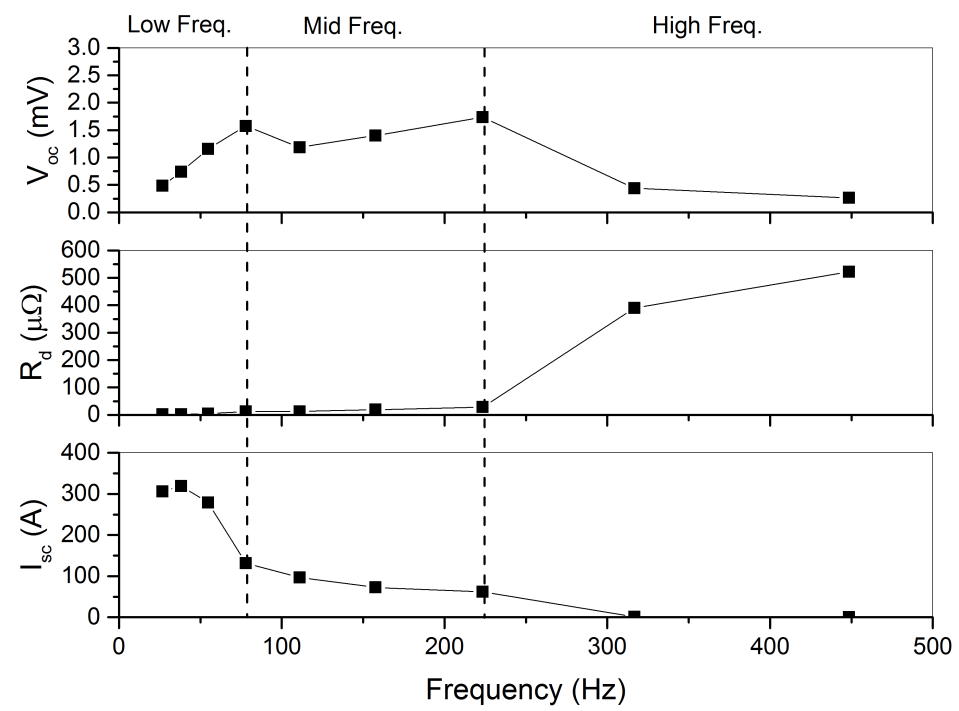

(a) Stator I

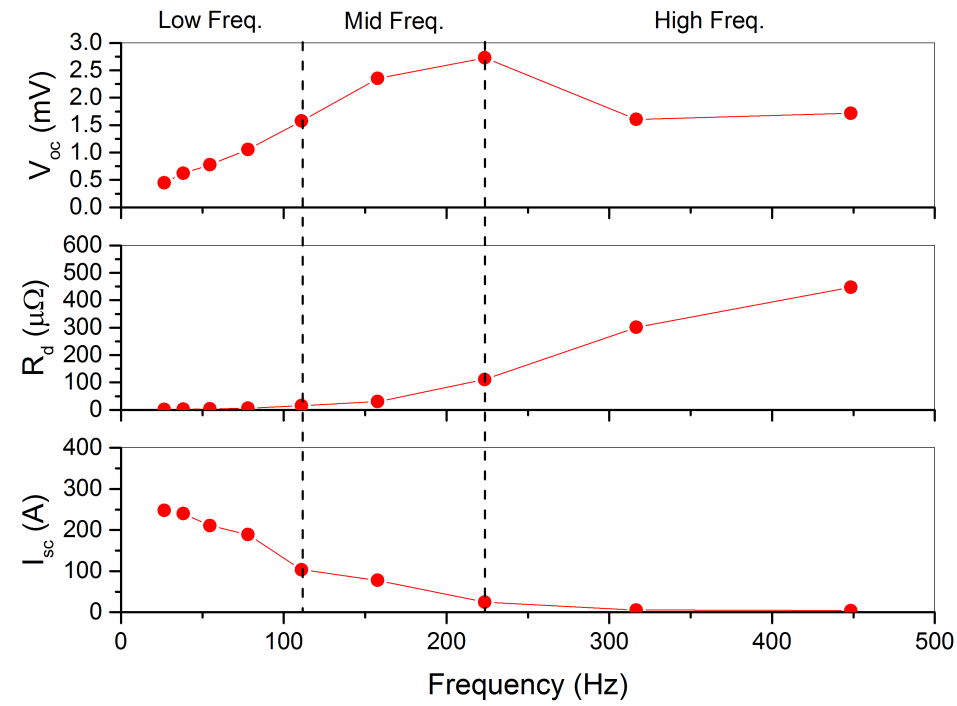

(b) Stator II

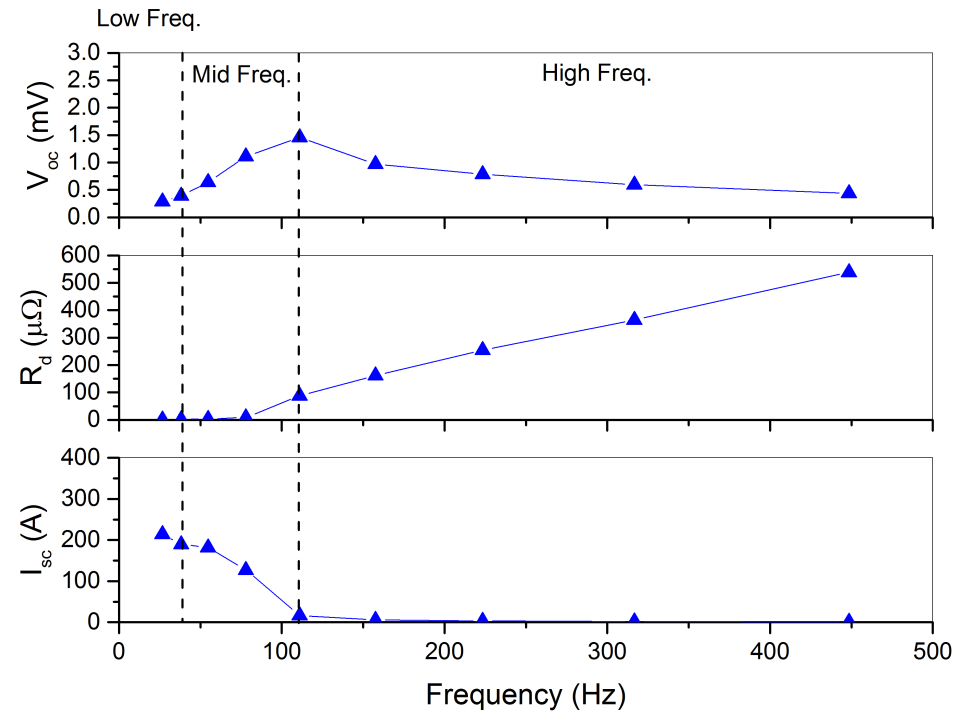

(c) Stator III

Figure 4.24: Gen 2 FP performance. The lines joining the datapoints are guides to the eye. 
The rapid increase in $R_{d}$ at high frequencies might be due to the local heat quenching in the stator [7]. It occurs when the magnets are passing over the stator at high frequency and the eddy-current in the stator around the magnet spot exceeds $J_{c}$ [31]. As a result, these local regions around the magnet where the eddy-current exceeds $J_{c}$ become ohmic and produce power loss. However, the frequency at which the heat quenching starts to occur depends on the heat transfer rate between the stator and the liquid nitrogen and it varies from one experiment to another.

The slit stators appeared to be thermally less stable at high frequency compared with the full stator. One possibility is that the stators could have been damaged during the slitting process. It is also possible that the width of the individual stators were smaller and therefore the eddy-current exceeded $J_{c}$ at high frequency. [7].

\subsubsection{The AC Waveforms and the Operating Frequency Regimes}

Another useful piece of information which also indicates the operating frequency regimes of the FP is the open-circuit AC waveform measured at zero coil current. Figures 4.25 and 4.26 show the frequency-normalised open-circuit voltage $\left(V_{o c} / f\right)$ waveforms of stators I and III respectively measured at $77 \mathrm{~K}$. The normal conducting voltage waveform measured at room temperature $300 \mathrm{~K}$ is also included for reference. The letter $\mathrm{M}$ denotes the rotor angle at which one of the the nine magnets was located at the centre of the stator. 


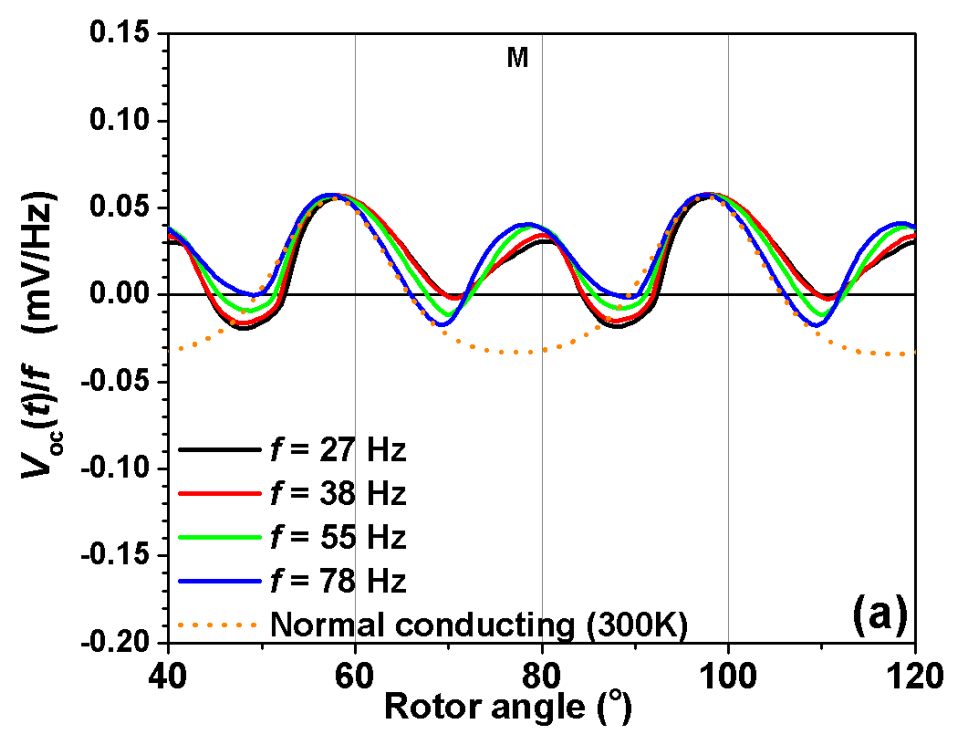

(a) Low Frequencies

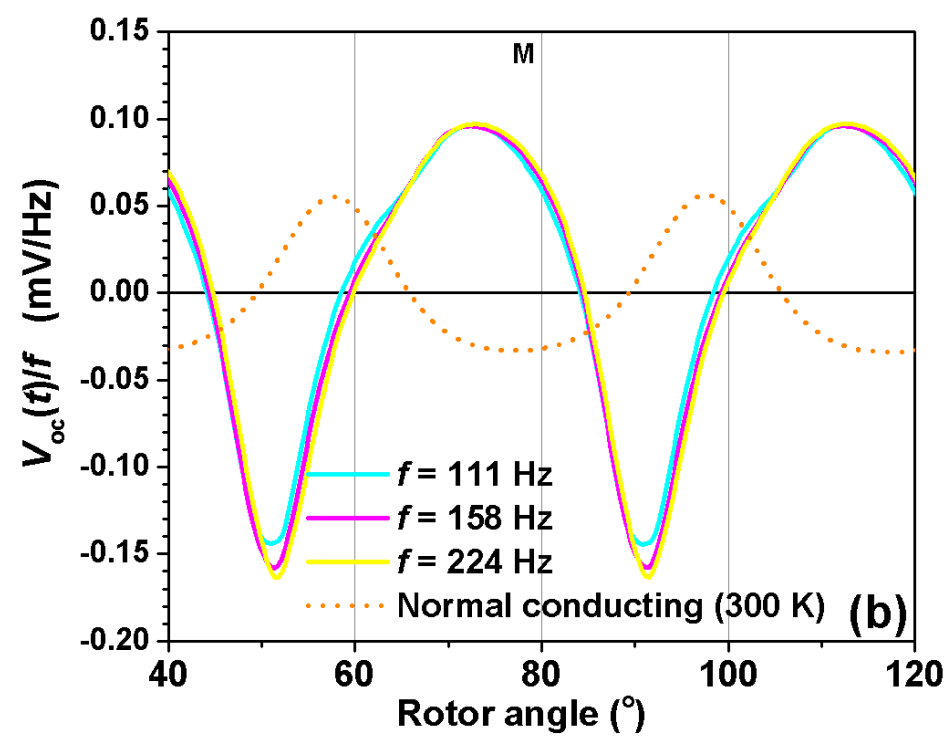

(b) Mid Frequencies

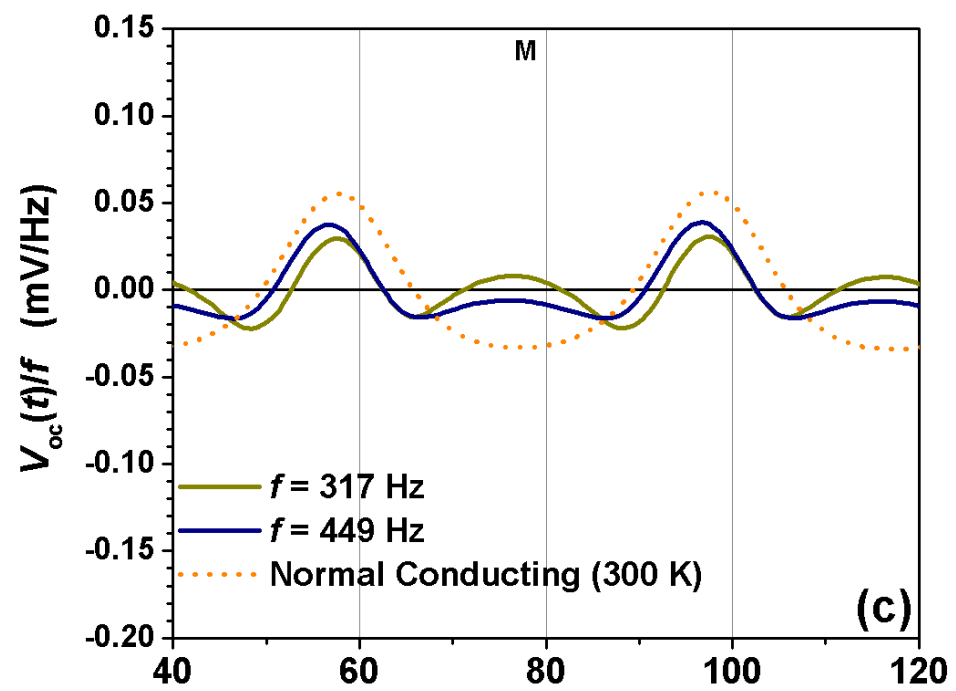

(c) High Frequencies

Figure 4.25: Stator I frequency-normalised open-circuit voltage $\left(V_{o c} / f\right)$ waveforms. Published in:[7]. 


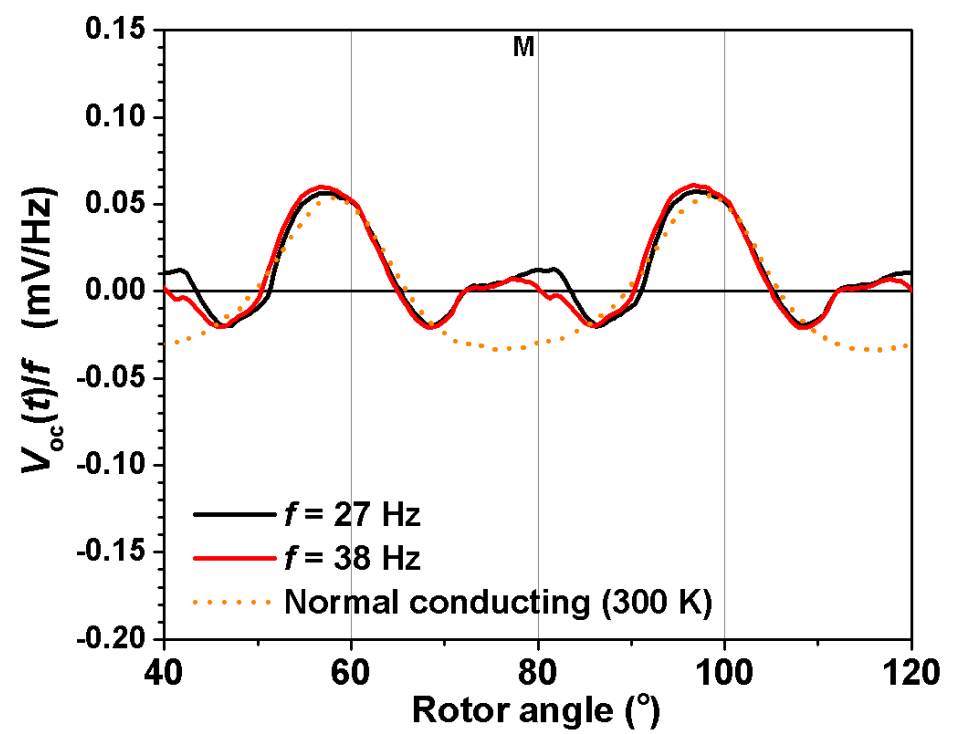

(a) Low frequencies

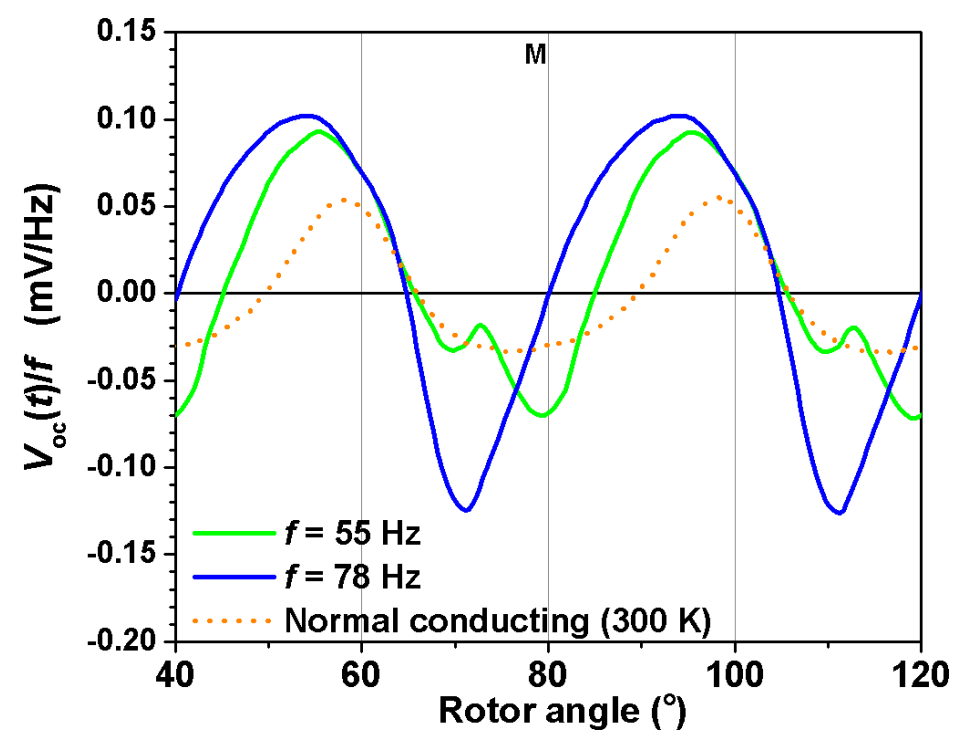

(b) Mid frequencies

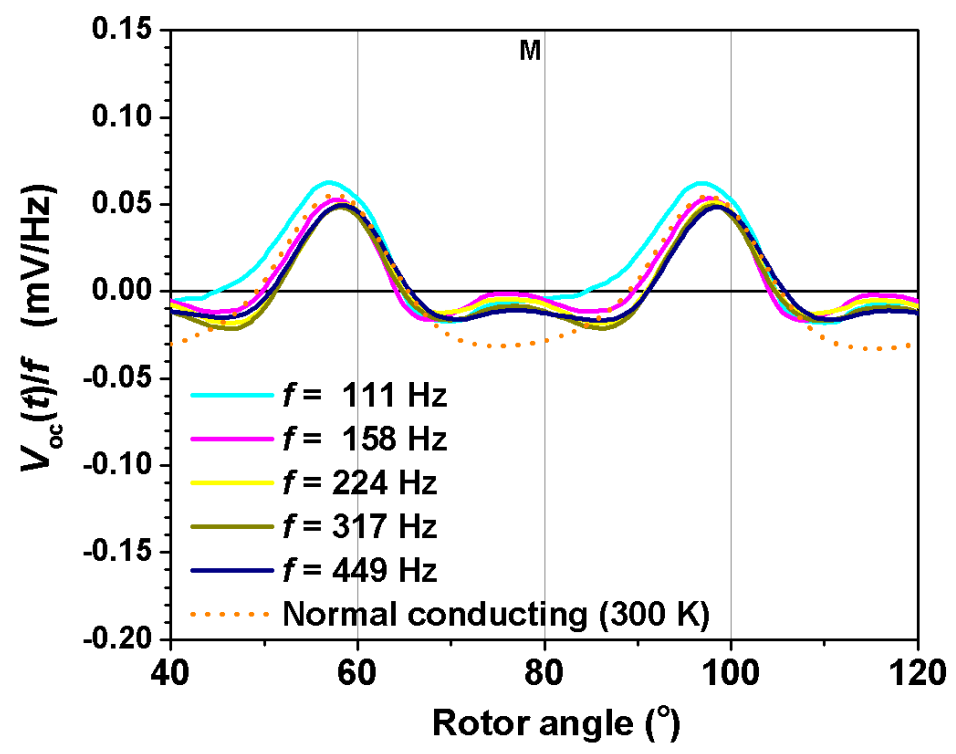

(c) High frequencies

Figure 4.26: Stator III frequency-normalised open-circuit voltage $\left(V_{o c} / f\right)$ waveforms. Published in:[7]. 
The waveforms are grouped into the three operating frequency regimes as described in the previous section. In the low frequency regime (4.25a and $4.26 \mathrm{a})$ it can be seen that the shape of the superconducting waveforms are different from the normal conducting reference waveform. The superconducting waveforms were partially rectified when the magnets were crossing over the stator (rotor angle from $67.0^{\mathrm{O}}$ to $88.8^{\mathrm{O}}$ ). In order to eliminate the voltage induced in the remainder of the voltage loop (e.g. stators voltage tap leads), the $300 \mathrm{~K}$ waveforms are subtracted from the $77 \mathrm{~K}$ waveform. Figure 4.27 shows an example of the subtracted waveform $\Delta \mathrm{V} / \mathrm{f}$ measured at $27 \mathrm{~Hz}$ with stator III. It shows that contributions to the DC output of the FP occurred only when the magnets (indicated by the letter M) were crossing over the stator. This is consistent with the theory presented in section 2.3.2 which explained the origin of the DC output of a HTS dynamo type FP.

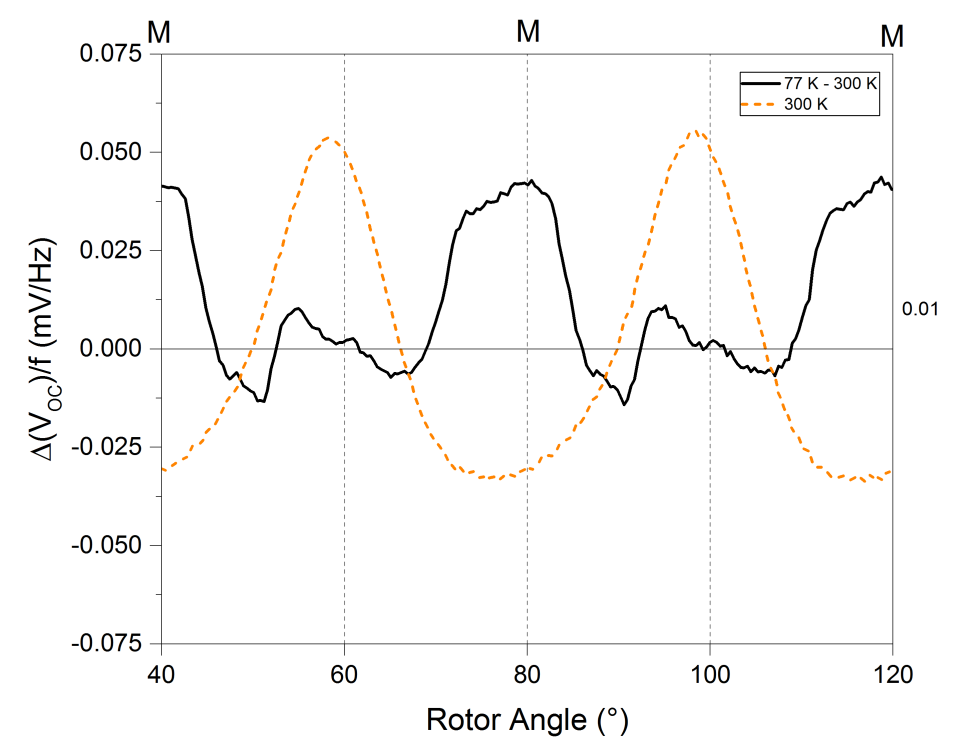

Figure 4.27: Stator III $\Delta V / f$ at $27 \mathrm{~Hz}$

At high frequencies $(4.25 \mathrm{c}$ and $4.26 \mathrm{c})$ the voltage shapes have similar form as the voltage shapes obtained at low frequencies. However, the DC output voltage of the FP is much lower. A possible explanation is that at high frequencies the stators were thermally quenched due to the circulating eddy-current being much larger than $J_{c}[7]$.

Figures $4.25 \mathrm{~b}$ and $4.26 \mathrm{~b}$ show the waveforms when the FP was in the transition from thermally unquenched to quenched state. It is interesting to note that in this mid-frequency regime the amplitude of the voltage is substantially higher compared with the low and high frequency regimes. There is no firm understanding of the underlying mechanism for this effect but it might be "due to an additional self-field $\mathrm{d} \Phi / \mathrm{dt}$ term caused by the circulating eddy currents within the wide stator wire" [7]. Further investigations are required in order to understand this effect properly. 


\subsubsection{Bi-stable Mode}

Another interesting observation on the behaviour of the slit stator experiments is that in some cases the FP was operating in two different stable modes. This was observed in the mid-frequency regime where the FP produced two $I-V$ curves, each of which had a different set of $V_{o c}, R_{d}$ and $I_{s c}$ values. An example is shown in figure 4.28 for stator III operating at 111 Hz. It shows that the FP was initially operating in the fourth quadrant in the unquenched mode and switched to the partially-quenched mode after the rotor had reached full speed. It then switched to and fro in between the two modes in the first and second quadrants. The $\mathrm{AC}$ waveforms measured in the two modes also differ in shapes and amplitude. It can be seen that the waveform amplitude in the quenched mode is smaller, which is consistent with the finding in the previous section. Note that the shapes and amplitudes of the unquenched mode waveforms are very similar to the mid-frequency open-circuit waveform shown previously (See figures $4.25 \mathrm{~b}$ and $4.26 \mathrm{~b}$ ). The reason is that that the FP was operating at mid-frequency of $111 \mathrm{~Hz}$.

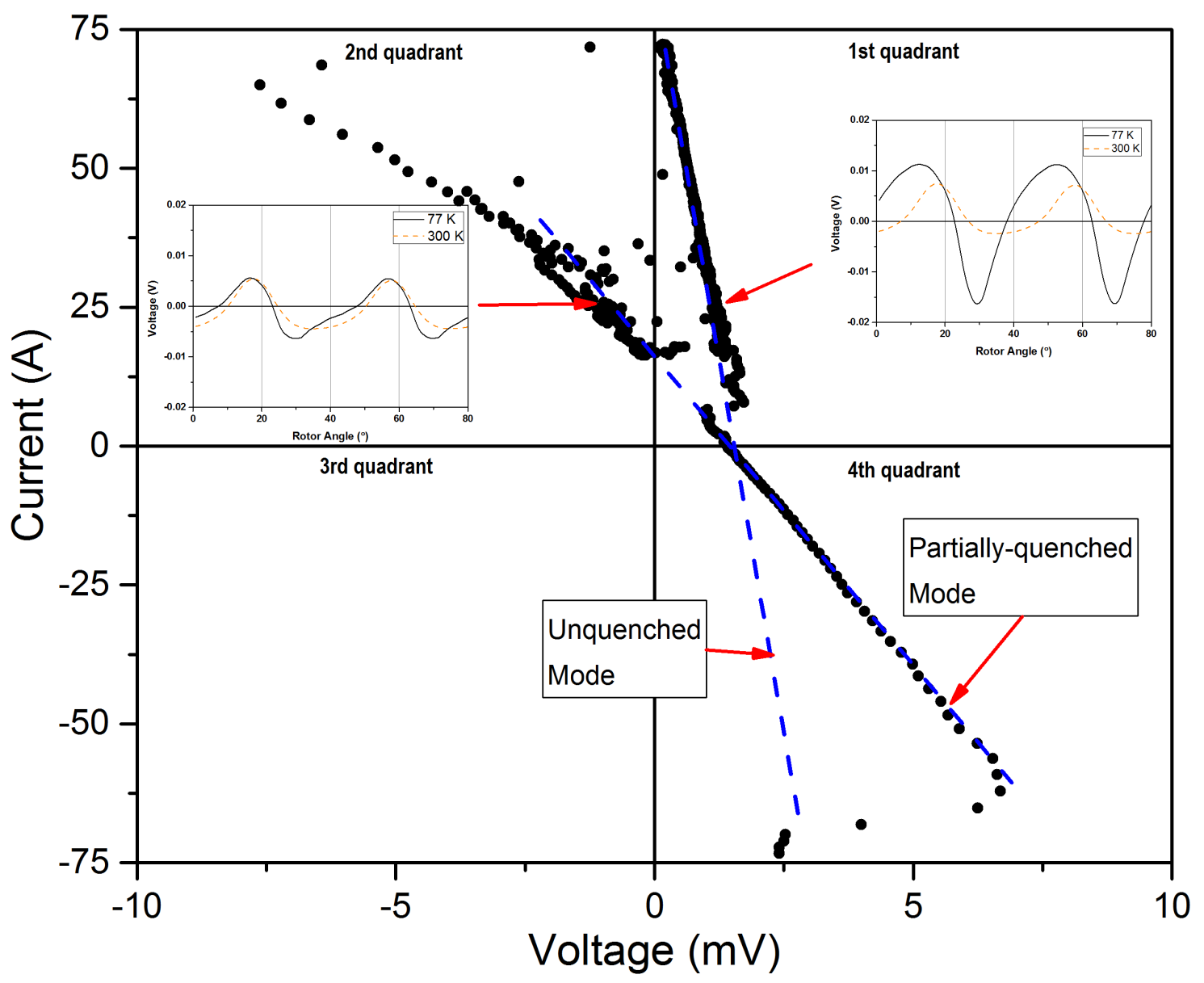

Figure 4.28: Stator III bi-stable mode at $111 \mathrm{~Hz}$

Figure 4.29 shows the time plot of the current, voltage and power of the FP operating in the bi-stable mode in figure 4.28. The grey colour sections shows the time when the FP was dissipating power and this corresponds to the second quadrant of the $I-V$ plot. 

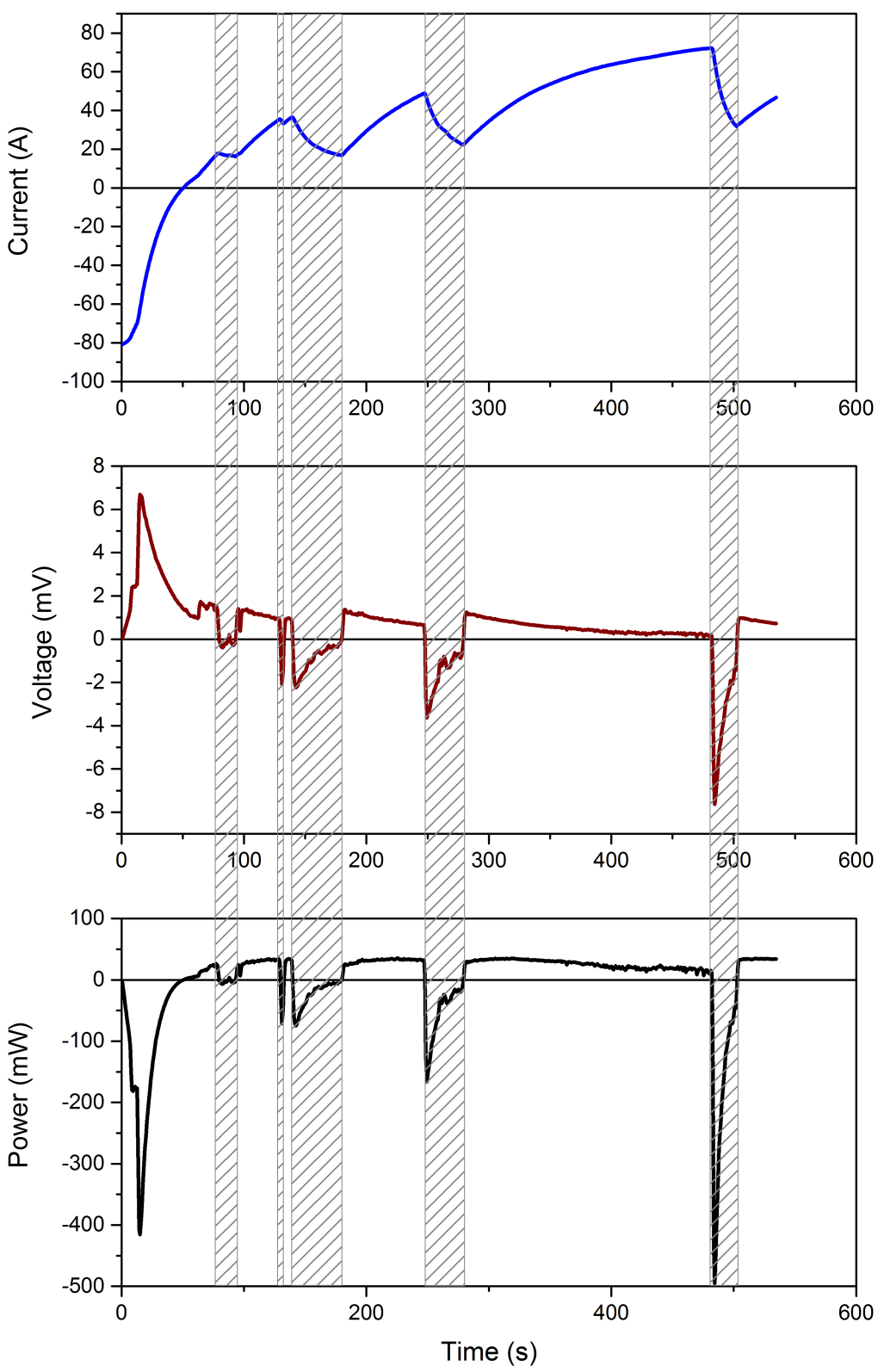

Figure 4.29: Stator III time plot at $111 \mathrm{~Hz}$. The grey colour sections shows the time when $V_{F P}$ was negative and the FP was dissipating power.

\subsubsection{Summary}

Three types of stators of different widths were used in the Gen 2 FP system experiments. The Gen 2 dynamo type HTS FP was capable of generating DC output in superconducting state due to the partial rectification of the $\mathrm{AC}$ voltage waveforms measured across the stator in superconducting state. The contact resistances in the FP dissipate power and the total value can be determined from a linear curve fit to the decay current data. The performance of the FP was studied and three distinctive operating frequency regimes were observed. At high frequency, the $R_{d}$ of the FP increased dramatically and this resulted in substantial reduction in the output current. The increase in $R_{d}$ is believed to be due to local heat quenching in the stator caused by large circulating eddy-current around the magnet spot. 
The slit stators were thermally less stable compared with the full stator. It has been shown that the $\mathrm{AC}$ waveforms in different $\mathrm{FP}$ operating frequency regimes differ in shapes and amplitudes. At certain mid-frequencies the FP switched the operation between two stable modes, each with a different set of $V_{o c}, R_{d}$ and $I_{s c}$ values. 


\section{Chapter 5}

\section{Gen 4 FP System Experimental Design and Commission}

\subsection{RRI Fourth Generation Flux Pump (Gen 4 System)}

The fourth generation (G4) FP system is a proof-of-concept design, with the intention to investigate whether this design would produce a DC current and characterise the FP performance at different operating temperatures. In this chapter, the design, installation, construction and commission of the system will be discussed. Apart from the mechanical design of the FP, which was completed prior to the project, all the works presented in this chapter were performed as requirements of this Master thesis.

\subsubsection{The Gen 4 Cryogenic System}

In this project, the superconductor and the test rig were cooled to about $30 \mathrm{~K}$ using a cold head via thermal conduction. The cold head and the rig assembly were located in a vacuum chamber and the air was pumped out by a turbo pumping system.

The vacuum chamber was manufactured by GNS Science New Zealand and it is shown in figure 5.1 . 


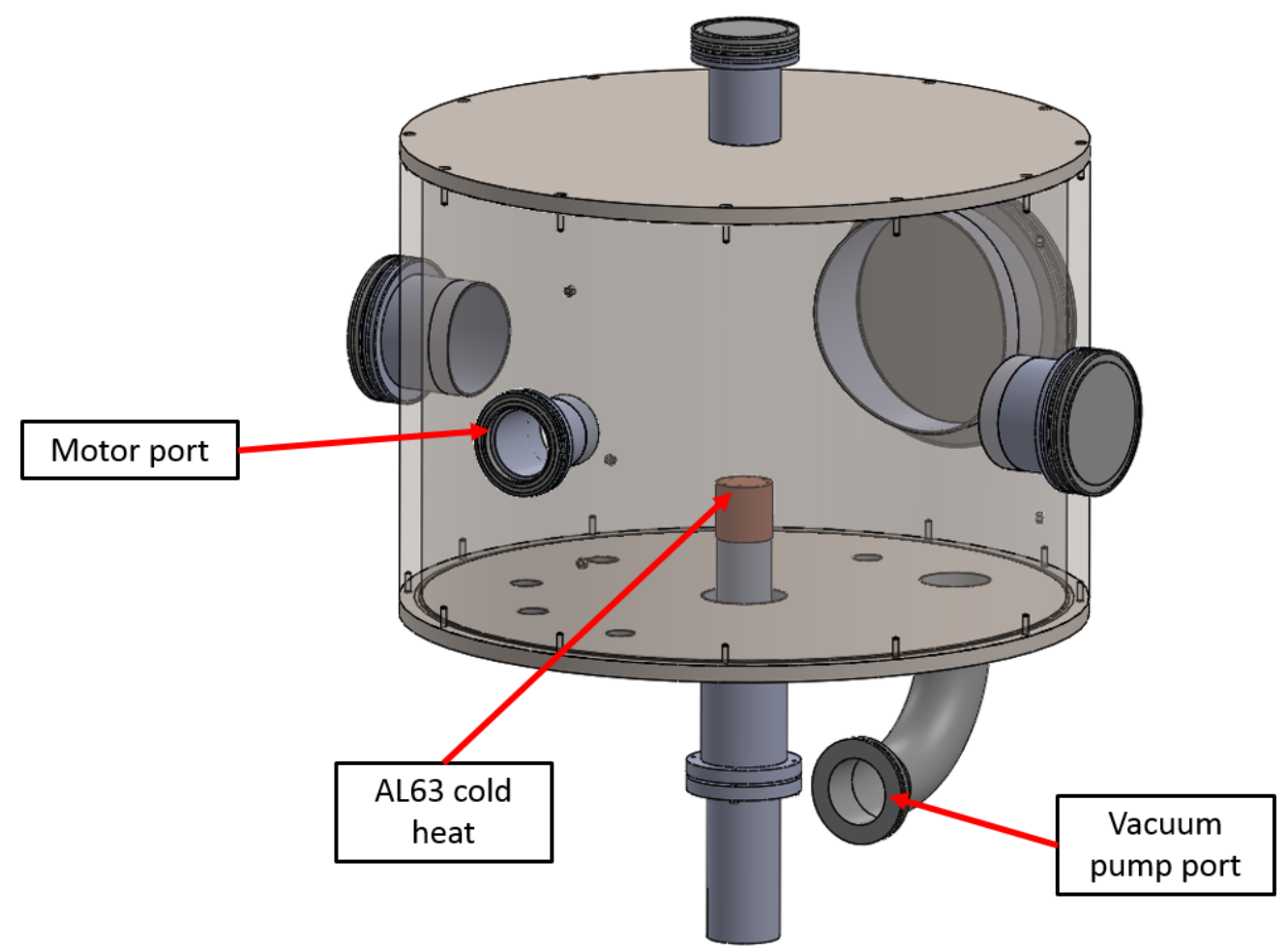

Figure 5.1: Gen 4 vacuum chamber. RRI Solidworks model.

The cold head was attached to a cryogenic refrigeration system (Cryomech AL63), which employed a closed loop helium expansion cycle for the cooling of the cold head [9]. The cooling capacity of the system is shown in figure 5.2. It shows that the cooling power decreases approximately linearly with temperature. At $30 \mathrm{~K}$ the cooling power is about 15 W.

\section{This content is not available. Please refer to the print version for access.}

Figure 5.2: AL63 Cryorefrigerator Capacity Curve. Source:[8].

The turbo pumping system is the HiCube Classic 80 series [32] manufactured by Pfeiffer 
Vacuum. It can deliver an ultimate pressure of $1 \times 10^{-7}$ mbar.

\subsubsection{The HTS FP Construction}

Figure 5.3 shows the construction of the fourth generation HTS FP system. It consists of a sample test rig and a HTS FP circuit. The sample test rig, which was designed by a student in a separate project, secures the FP circuit in the vacuum chamber and is intended to enable cooling to about $30 \mathrm{~K}$. The cooling is done via thermal conduction by attaching a copper cooling plate to the cold head, which is part of the Cryomech AL63 system. A thin layer of Apiezon $\mathrm{N}$ grease (cryogenic vacuum grease) was applied on the interfaces between the cooling plate and the cold head.

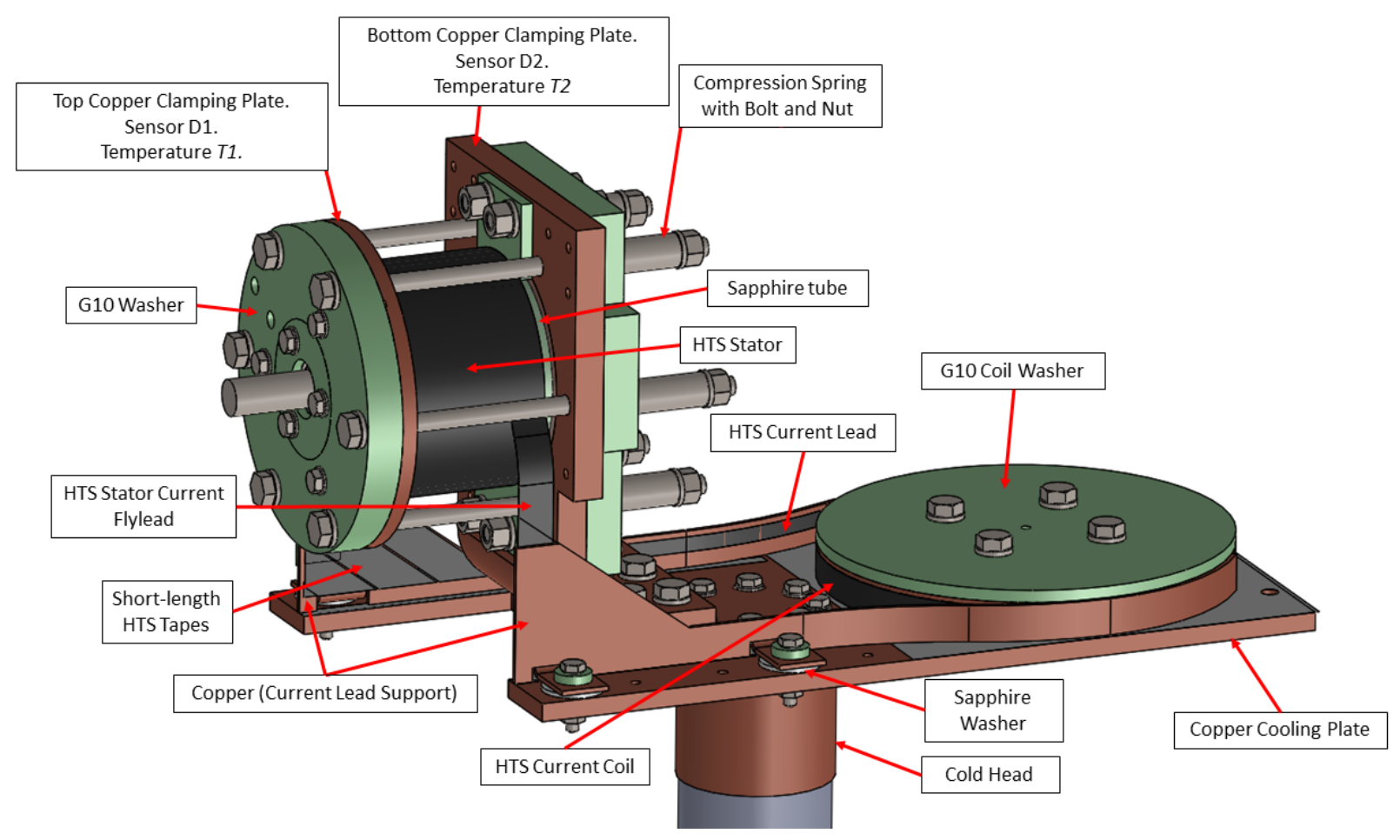

Figure 5.3: The fourth generation HTS FP attached to the sample test rig. The location of the main temperature sensors D1 and D2 are also shown in the diagram. RRI Solidworks model.

The cooling plate holds and cools the HTS current coil, the copper current-lead supports and the HTS stator assembly. The functions of the sapphire washers are to provide electrical isolation between the current-lead supports and the cooling plate, and to provide good thermal conduction between them. For good thermal conduction, a thin layer of Apiezon N grease was applied to the interfaces between the components.

The bottom copper clamping plate of the stator assembly serves as a thermal conduction path between the cooling plate and the stator. The top clamping plate, bottom clamping plate and the compression springs hold the cylindrical sapphire tube in position.

The sapphire tube provides a surface on which the stator wraps around and forms a cylindrical shape. Sapphire $\left(\mathrm{Al}_{2} \mathrm{O}_{3}\right)$ was chosen because it is a good electrical insulator $\left(10^{16}\right.$ ohm-cm @ $\left.25{ }^{\circ} \mathrm{C}\right)[34]$ and thermal conductor $\left(12000 \mathrm{~W} / \mathrm{m} / \mathrm{K} @ 25^{\circ} \mathrm{C}\right)$ [11]. 


\subsubsection{HTS Stator Assembly}

Figure 5.4a shows the cross-section of the HTS stator assembly. It consists of a sapphire tube, a stator, a rotor and two copper clamping plates. The cylindrical rotor was made of aluminium and was designed to hold permanent magnets of different size and orientations. A $12 \mathrm{~mm}$ x $12 \mathrm{~mm}$ x $12 \mathrm{~mm}$ Nd-Fe-B magnet was used in this project and it was oriented so that the north pole, and therefore the magnetic field, pointed towards the stator. The largest magnet size available was chosen for the strongest magnetic field strength (see section 4.1 for the discussion on the effect of magnet size relative to stator width on FP performance). The flux gap measured from the surface of the magnet to the stator was about $4.5 \mathrm{~mm}$. The rotor shaft was coupled to an external servo motor at room temperature via a carbon coupling tube. A Hall sensor type HHP-NP supplied by AREPOC [1] was installed in close proximity to the magnet to detect its position and provide a timing signal to the acquisition software. 


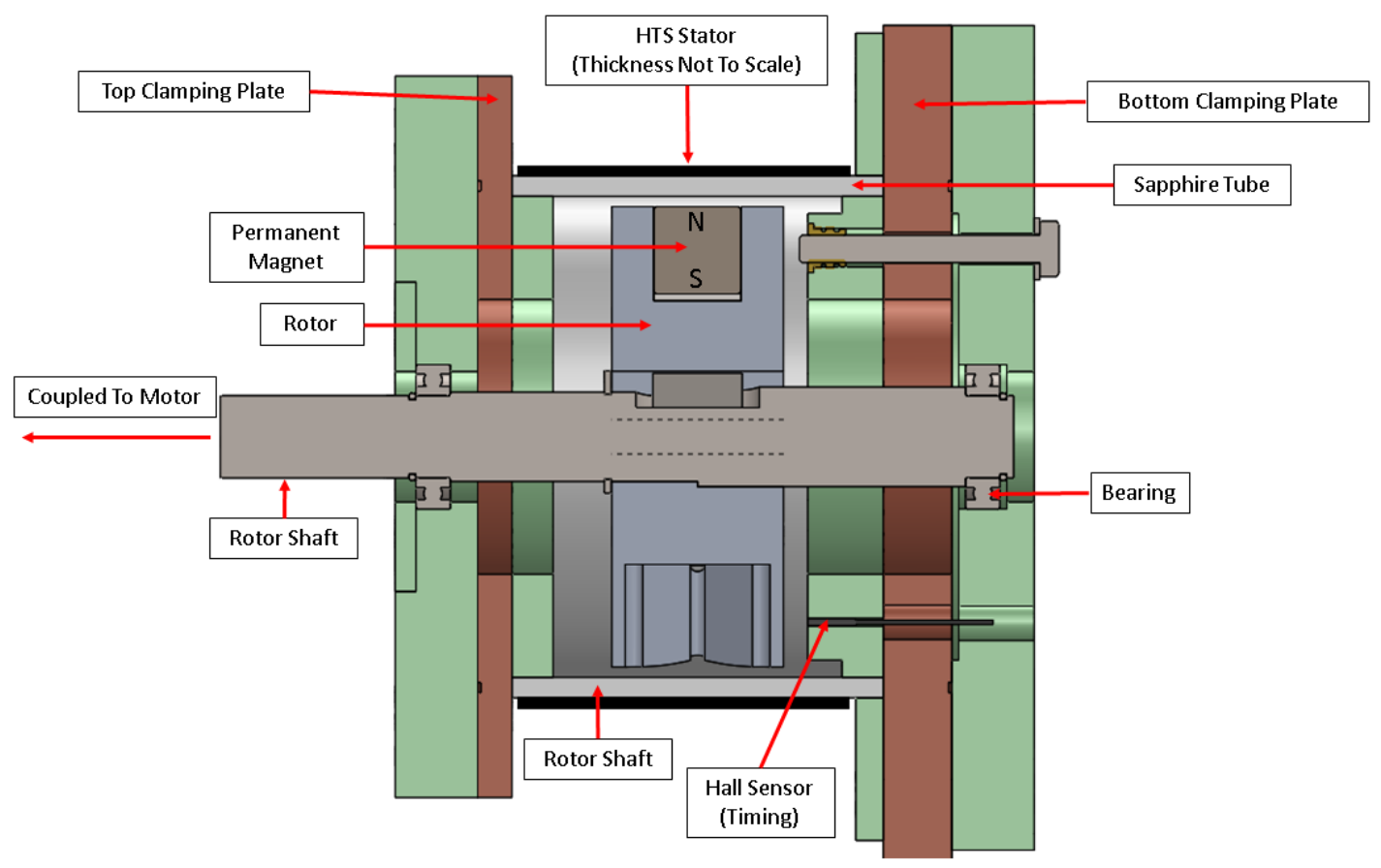

(a) Cross-section view

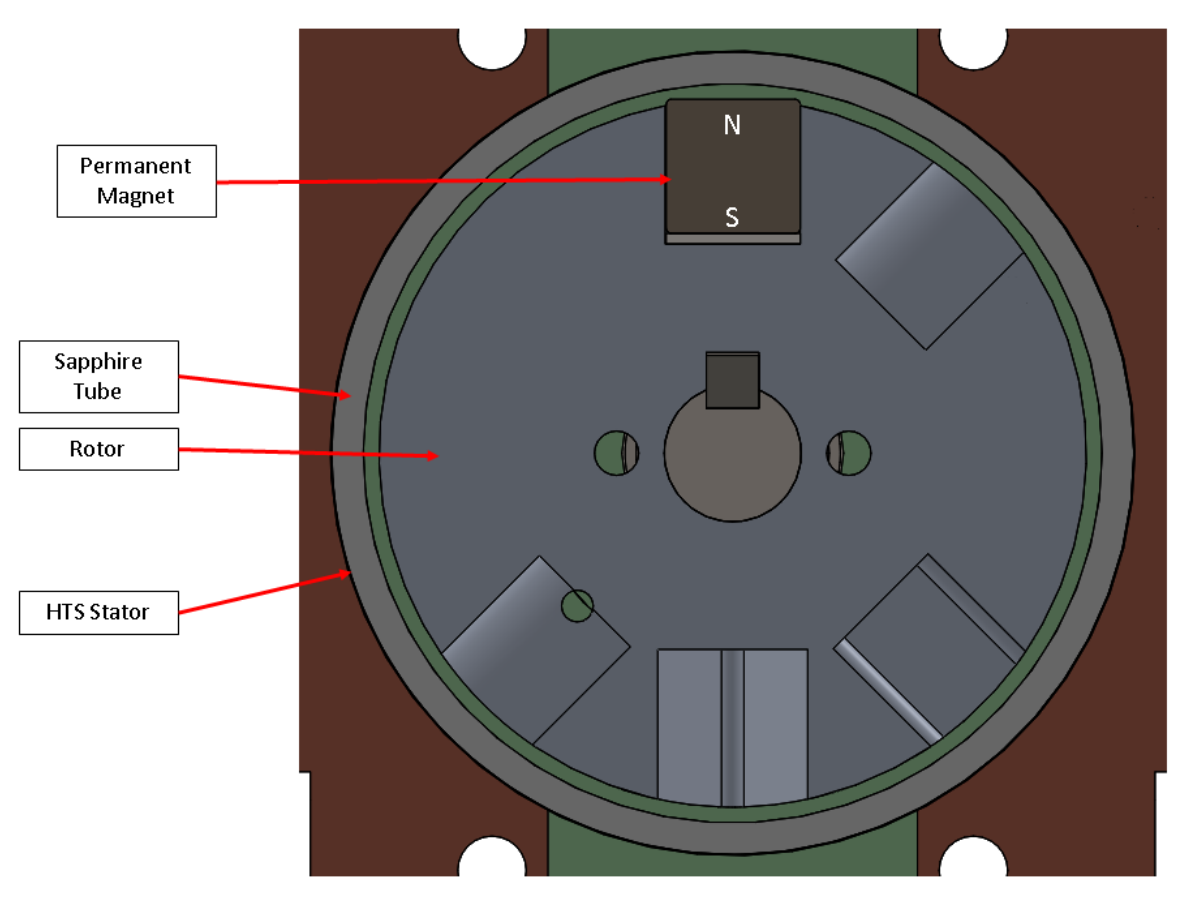

(b) Front view

Figure 5.4: Stator assembly cross section view. RRI Solidworks model.

\subsubsection{HTS FP Circuit}

Figure 5.5 shows the construction of the HTS FP circuit. Note that all components, except for the rotor, have been removed from the drawing for clarity. It consists of a HTS stator, which is connected to a double-pancake coil via two sets of flyleads and current leads. The stator is made of a $46 \mathrm{~mm}$ wide HTS tape supplied by AMSC. It has a layer of YBCO superconducting material deposited onto a hastelloy substrate and covered by a silver protective 
layer. The thickness of the YBCO and silver layers are about 1 and $8 \mu \mathrm{m}$ respectively and the $I_{c}$ is $225 \mathrm{~A} / \mathrm{cm}$, measured at $77 \mathrm{~K}$ at $1 \mu \mathrm{V} / \mathrm{cm}$ criterion. The current coil is a double pancake HTS coil manufactured by HTS-110. The inductance of the coil is $1.97 \mathrm{mH}$ and the $I_{c}$ is $95 \mathrm{~A}$, measured at $77 \mathrm{~K}$ at $1 \mu \mathrm{V} / \mathrm{cm}$ criteria.

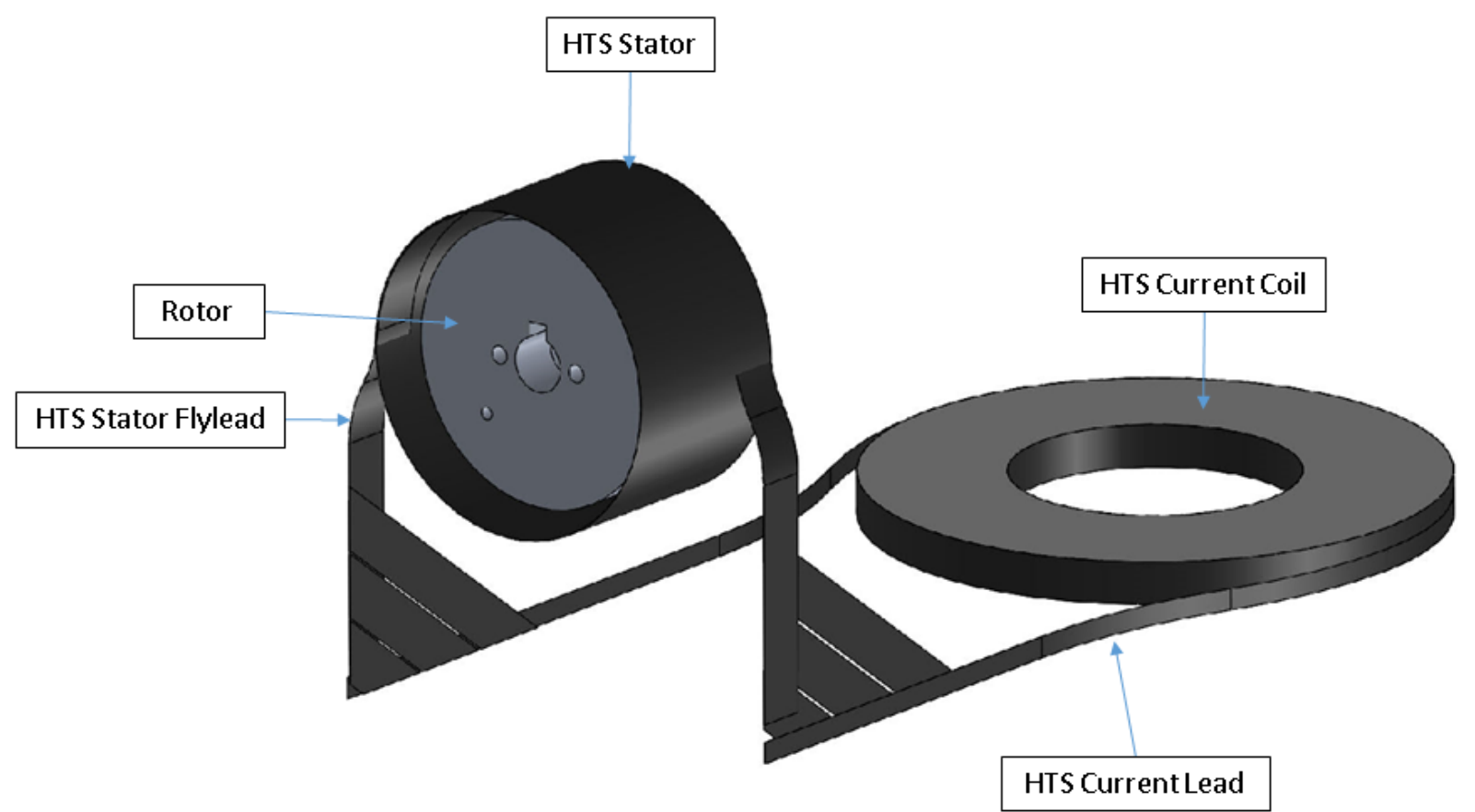

Figure 5.5: HTS FP Circuit. RRI Solidworks model.

\subsubsection{The Construction and Installation of the Stator and the Leads}

Figure 5.6 shows the process of making the stator. First, a tape of about $220 \mathrm{~mm}$ long was prepared and the back side (hastelloy) of the tape was applied with a very thin layer of Araldite 2 part-epoxy resin. The HTS tape was then rolled onto a sapphire tube and held in place with PTFE tape. The back side of the HTS touched the tube and the silver layer side faced outward. The expoxy was then cured in an oven set at $120^{\circ} \mathrm{C}$ for 24 hours. Once the epoxy was cured, the PTFE tape was removed and the ends of the HTS tape were joined together by soldering a HTS strip (made from the same HTS tape) over the gap. 


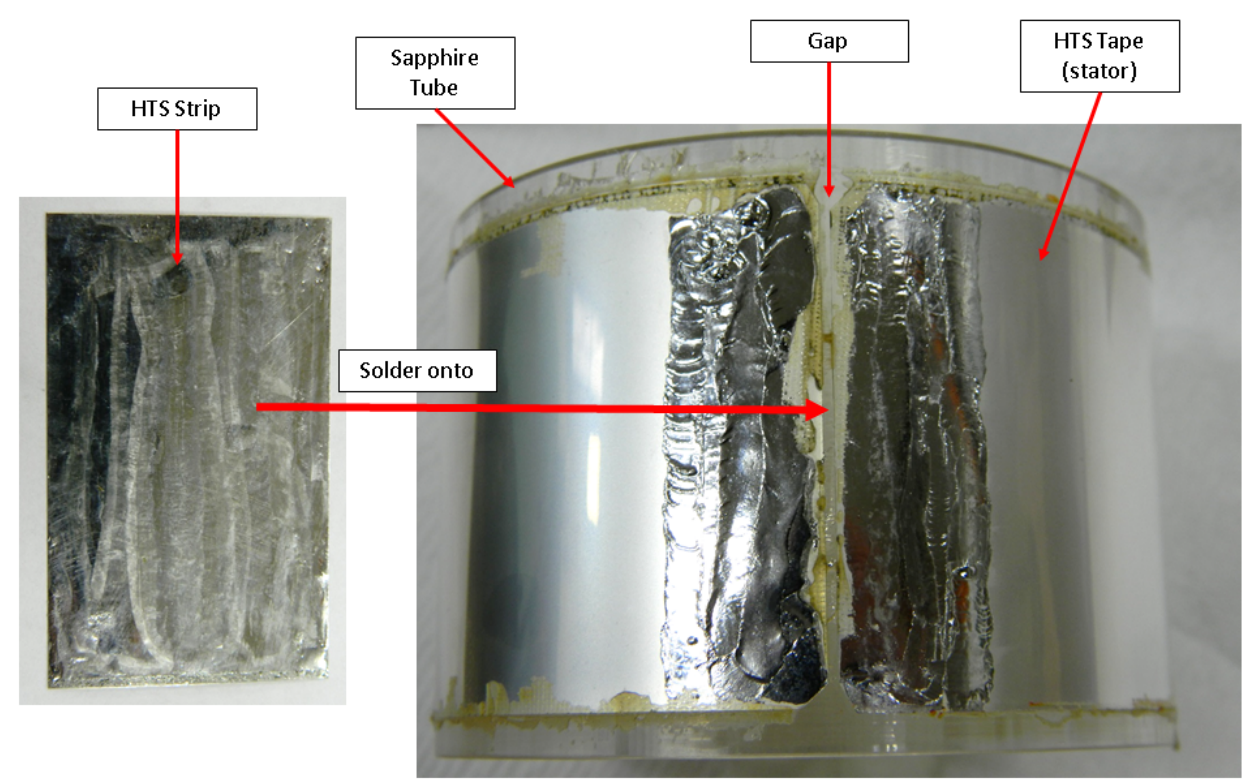

Figure 5.6: Mounting of the HTS stator onto the sapphire tube.

As shown in figure 5.5, the electrical (superconducting) connections between the HTS stator and the coil were made via 2 sets of stator flyleads and current leads. Each set was supported by a copper support piece, which provides mechanical support and thermal conduction. Both the flyleads and the current leads were made from $12 \mathrm{~mm}$ wide coppercoated YBCO HTS tape. The tape had an $I_{c}$ of about $300 \mathrm{~A}$ and it was manufactured by SuperPower. One end of the flyleads was soldered to the HTS coil and the other end to the support piece (hastelloy side touching the copper). This is shown in figure 5.7.

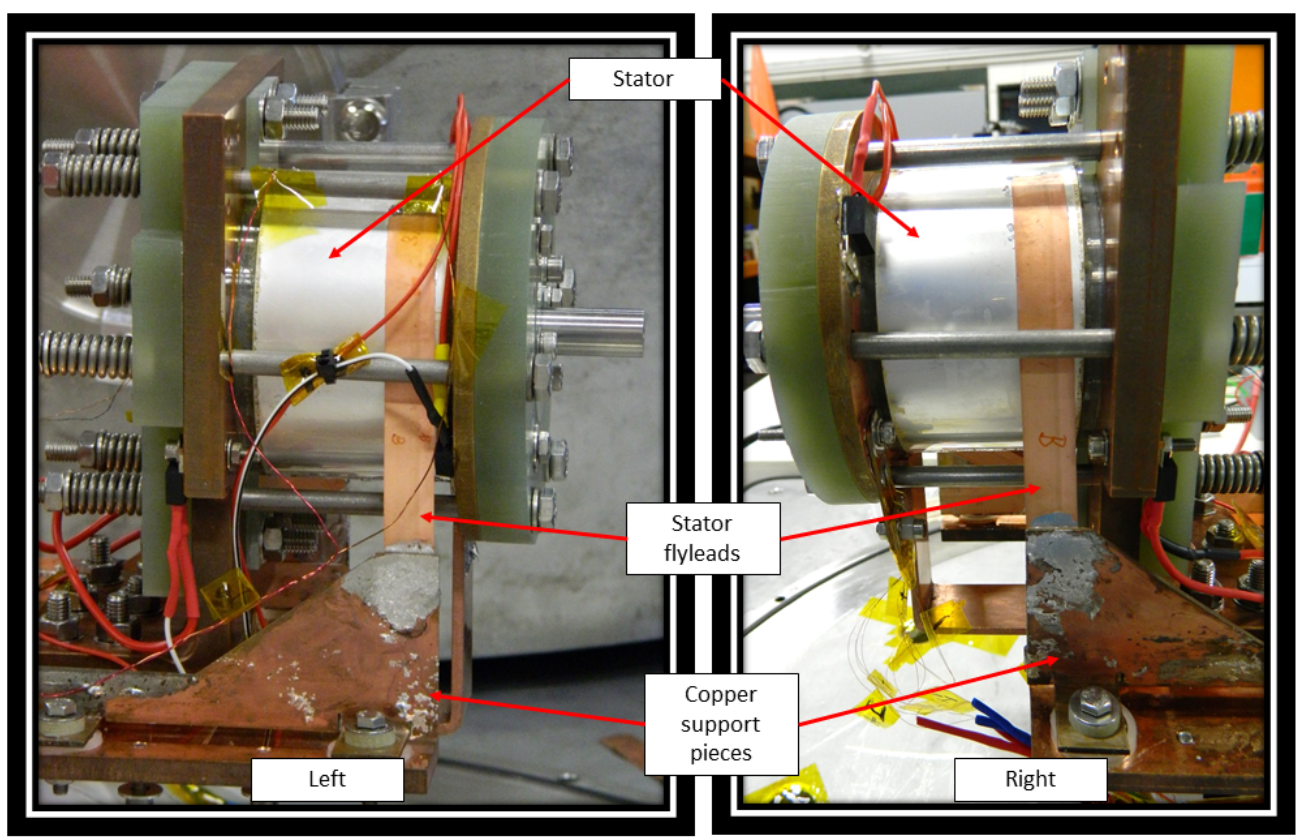

Figure 5.7: HTS flyleads soldered onto the copper support pieces and stator.

For the current leads the whole pieces were soldered directly onto the copper support pieces, with the back side (hastelloy) facing the copper. This is shown in figure 5.8 where 
the top photo shows the current lead attached to the support piece and the bottom photo shows the current lead, being soldered onto the HTS coil.
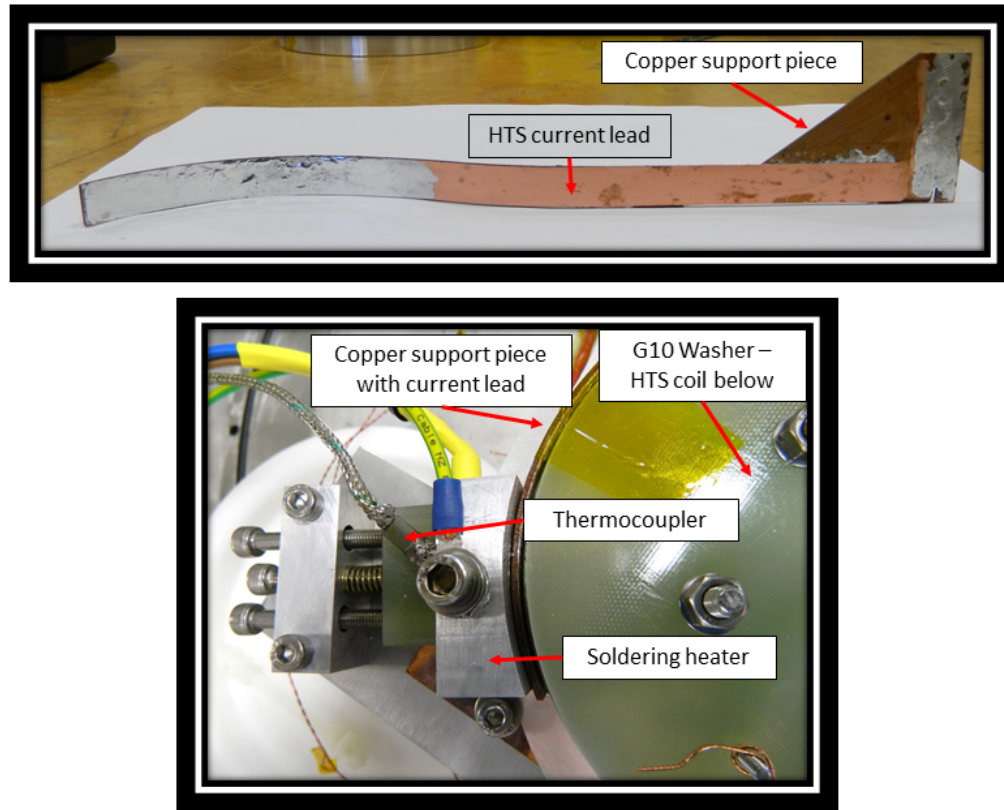

Figure 5.8: HTS current lead soldered onto a copper support piece and then onto the HTS coil.

The stator flyleads and the current leads were electrically connected via two pieces of short-length HTS tape, each of them was made from the same batch of the SuperPower tape. The connections are shown in figure 5.9.

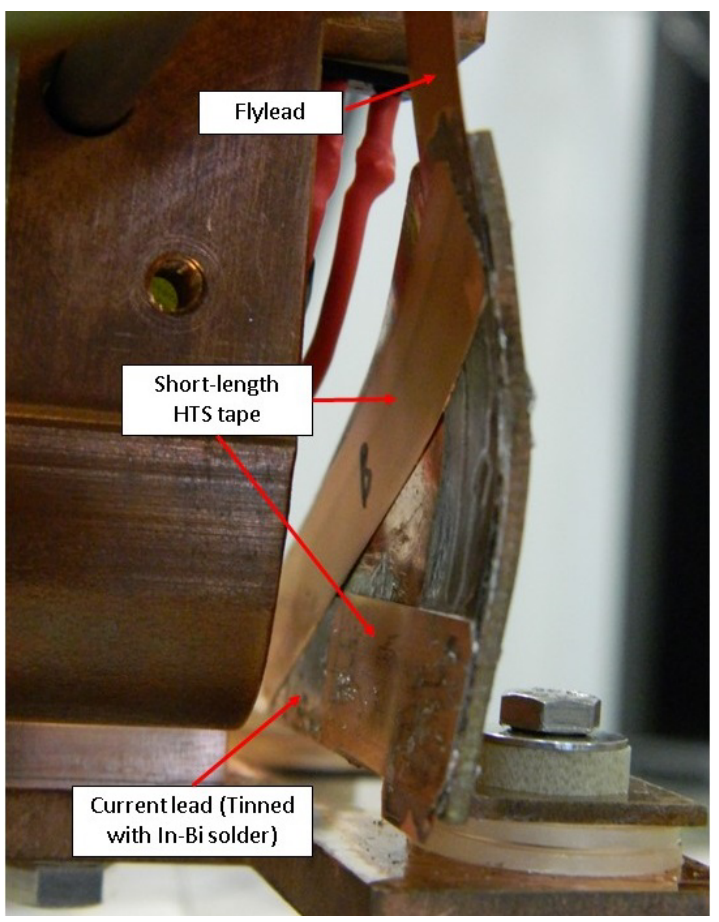

Figure 5.9: Connections between the flylead and the curent lead via two pieces of HTS tapes.

The same soldering heater shown in figure 5.8 was also used to solder the flylead onto the 
HTS stator. In order to have a good control on the heat generated by the heater, a power supply was custom built for it. This is shown in figure 5.10.

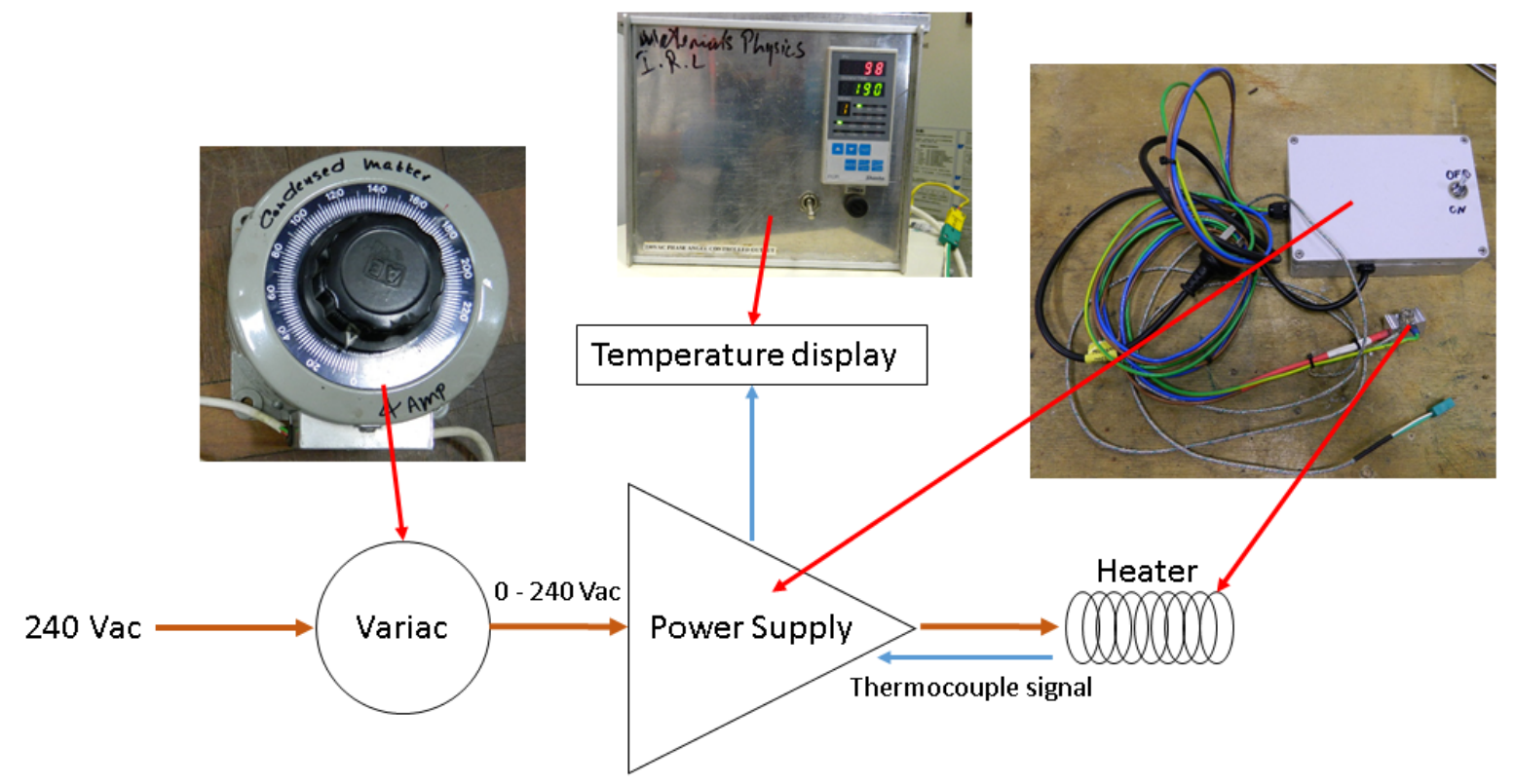

Figure 5.10: Soldering heater and the power supply.

\subsubsection{Temperature Monitor and Control of the Stator}

The cooling capacity curve of the AL63 cold head in figure 5.2 shows that the cooling power of the cold head at $80 \mathrm{~K}$ is about $50 \mathrm{~W}$. The cooling experiments (see section 5.2) conducted on the FP system showed that the lowest temperature achieved on the copper cooling plate was about $24 \mathrm{~K}$, and on the stator assembly was about $31 \mathrm{~K}$. Two 50-W heating elements were installed on the top and bottom clamping plates to bring the system to thermal equilibrium so that the temperature at the stator can be set to the desired level $(30-80 \mathrm{~K})$. A voltage-controlled constant-current source was designed and built in this project to control the output power of the heating elements (see appendix A for the design and construction of the heater power supply). The temperature on the plates were monitored using the Lakeshore Cryogenic silicon diode temperature sensors (DT-670 series [21]). The schematic of the temperature control and monitor is shown in figure 5.11. 


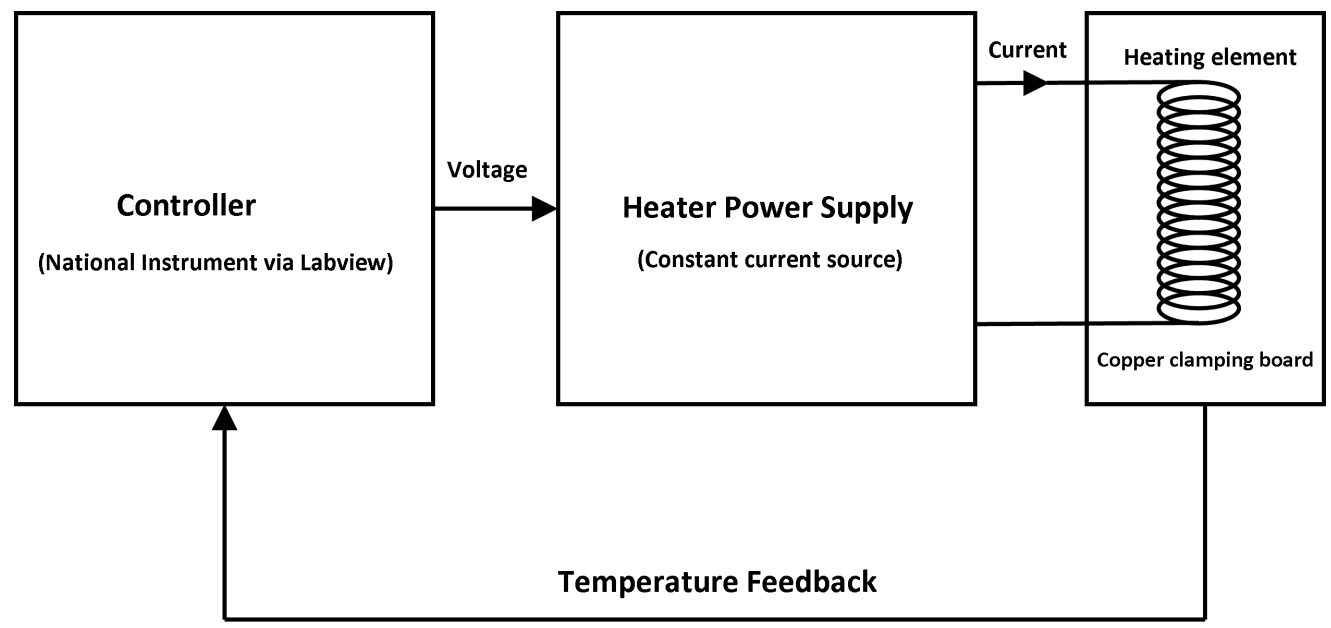

Figure 5.11: Stator temperature control and monitor.

Note that in this project the control voltage input to the power supply was set manually. However, a PID controller can be incorporated into the scheme if it is required.

The locations of the diode sensors and the heating elements are shown in figure 5.12. The diode on the top clamping plate is labelled as D1 and temperature as T1, and the diode on the bottom clamping plate is labelled as D2 and temperature T2. For consistency, these labels are fixed in this project.

In order to eliminate the error introduced by the high resistance in the leads, the 4-wire measurement method was used for the measurements of the sensors signals [20]. The sensor leads were also thermally anchored at various points to minimise the heat conduction from the ambient environment to the sensors [20].

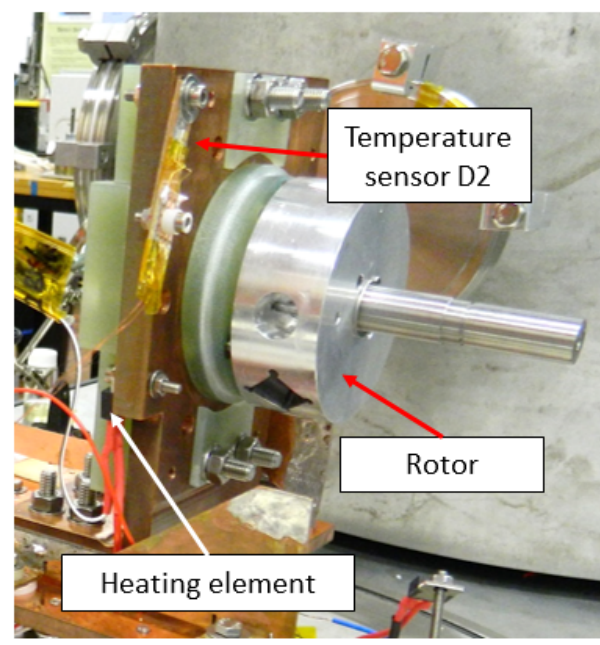

(a) Side view

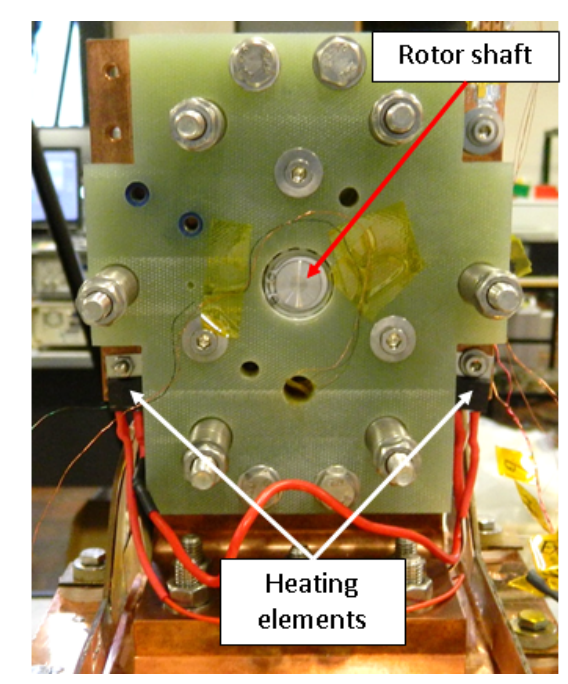

(b) Rear view

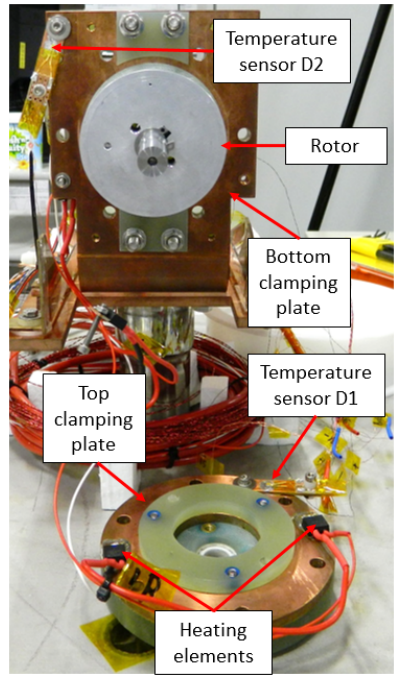

(c) Front view.

Figure 5.12: Temperature sensors and heaters on the stator assembly. (a) Side view showing the temperature sensor D2 and the heating element installed on the bottom clamping plate. The top clamping plate and the stator were removed. (b) Rear view showing the heating elements installed on the bottom clamping plate. (c) Front view showing the diode temperature sensor D1 installed on the top clamping plates. Two heating elements are installed on the the top clamping plate. The stator had been removed. 


\subsubsection{HTS Current Coil Assembly and Hall Sensor Calibration}

In the experiment, the HTS stator and the current coil would be connected in series. The current flow in the circuit would be monitored by using a high-linearity Hall sensor to detect the magnetic field at the centre of the current coil. The Hall sensor is an axial mounting type HHP-NA supplied by AREPOC and the specification can be found in literature [1]. The Hall sensor mounting in the coil assembly is shown in figure 5.13.

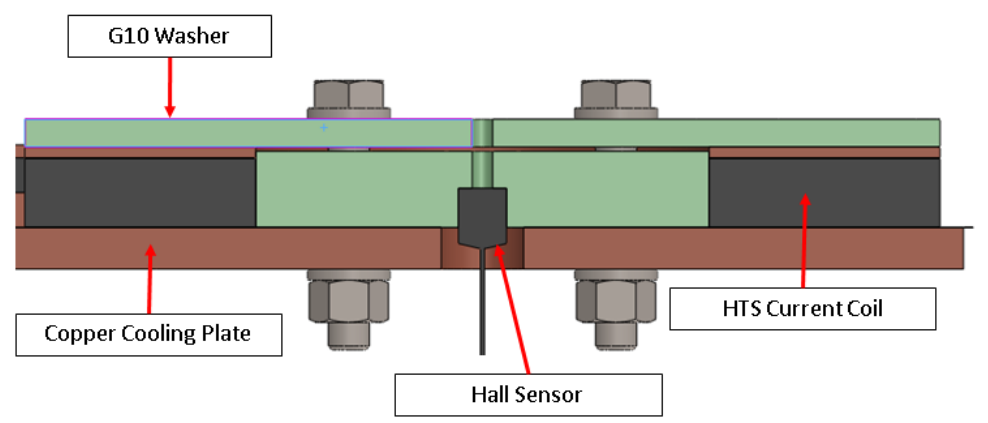

Figure 5.13: Gen 4 system HTS current coil assembly and the Hall sensor. RRI Solidworks model.

An experiment was conducted to obtain a conversion factor (calibration constant) of Hall sensor voltage to coil current. Figures $5.14 \mathrm{a}$ and b show the setup of the experiment. An external power supply was used as a constant current to set the coil current from zero to 95 $\mathrm{A}$, at a $5 \mathrm{~A}$ increment step. The Hall sensor converted the magnetic signal detected at the centre of the coil into a voltage signal. This signal was amplified and recorded by a NI-9205 analogue signal input module. The Yokogawa 7551 constant current source supplied a $20 \mathrm{~mA}$ excitation current to the Hall sensor and the Agilent 34420A meter monitored the voltage across the HTS coil to ensure that the current did not exceed the $I_{c}$ of the coil.

In order to protect the coil assembly from a huge thermal shock, an $N_{2}$ flow control setup was used to supply $N_{2}$ into the bath and cooled the assembly at a very slow rate via $N_{2}$ vapour cooling. This is shown in figure $5.14 \mathrm{c}$. 


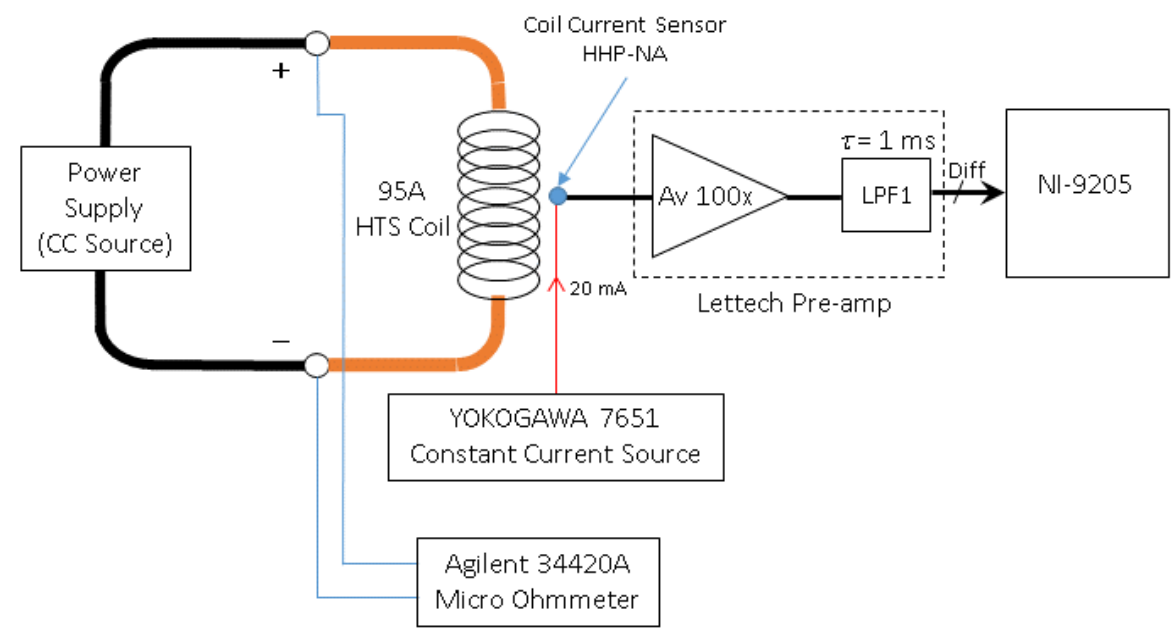

(a) Signal acquisition.

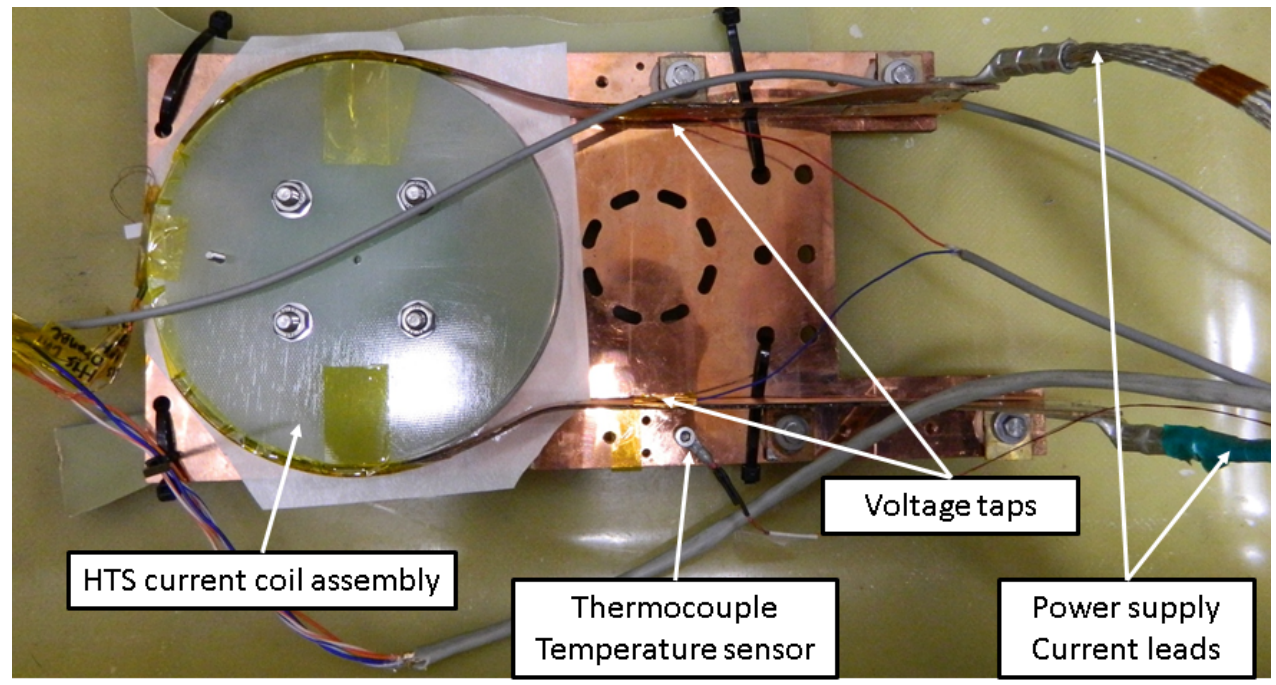

(b) Photo showing the experimental setup.

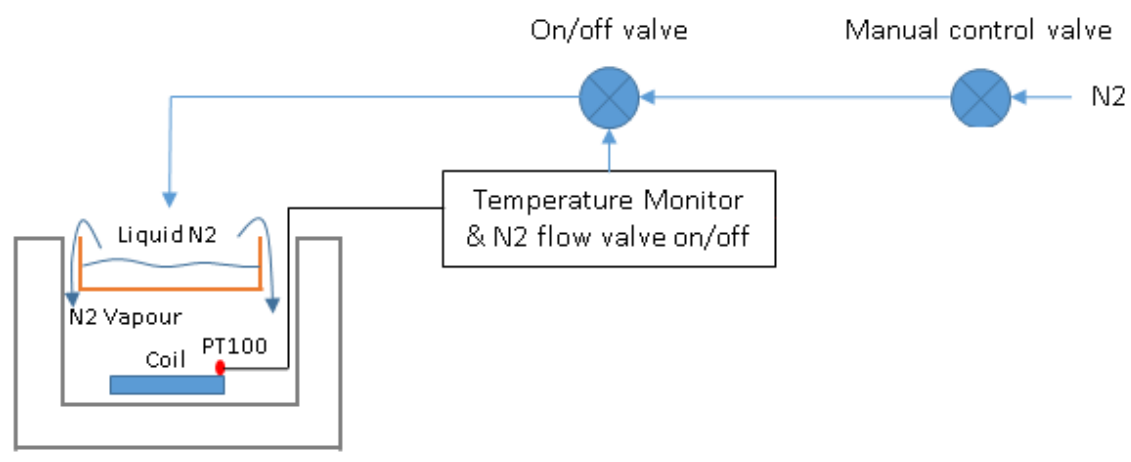

(c) Coil assembly temperature control.

Figure 5.14: Gen 4 system HTS current coil Hall sensor calibration.

The calibration curve obtained from the experimental data is shown in figure 5.15 and the calibration constant is $105.24 \mathrm{~A} / \mathrm{V}$. 


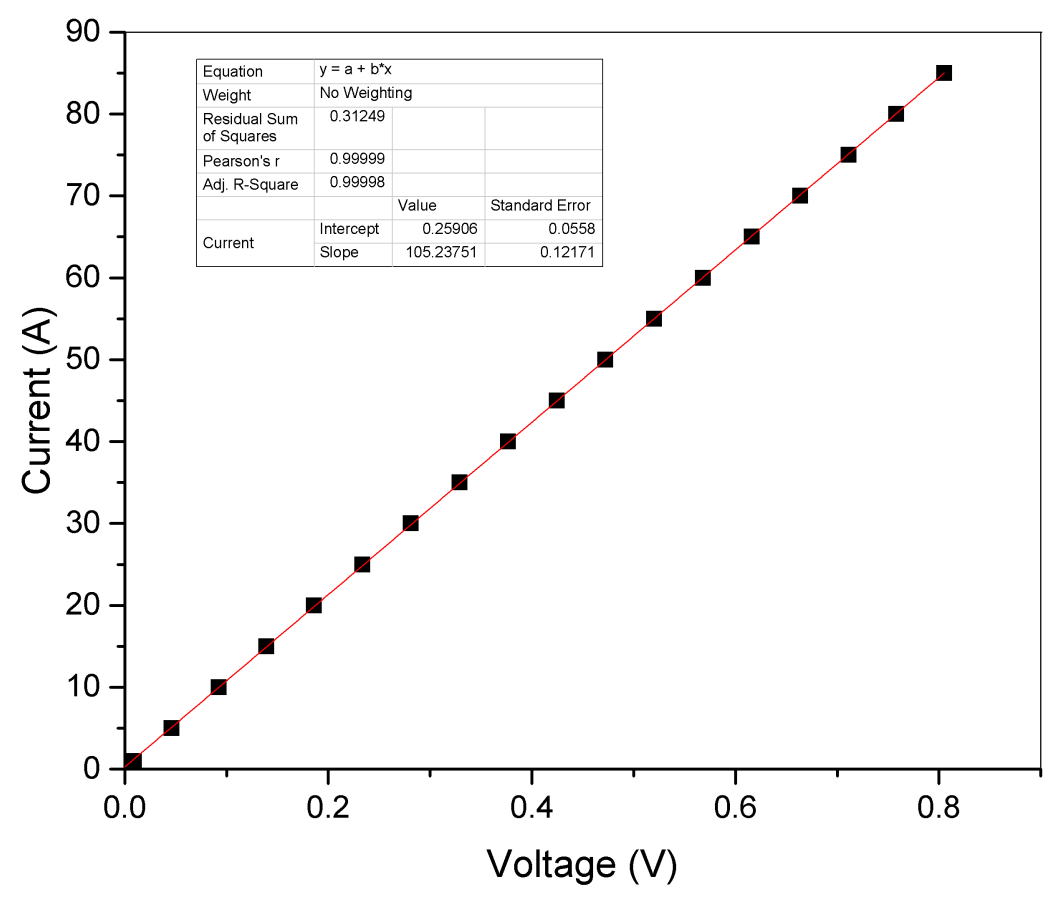

Figure 5.15: Gen 4 HTS coil Hall sensor calibration curve.

\subsubsection{Schematic Diagram and Signal Acquisition}

Figure 5.16 shows the schematic and signal acquisition of the G4 system. There is some similarity between the Gen 2 and Gen 4 systems (e.g. both have a current coil acting as the load) and therefore the Gen 2 Labview software was modified to suit this the Gen 2 system. However, it is important to note that the installation of the equipment and acquisition units of the Gen 4 system were done afresh, independent of previous works. In addition to that, some of the electronics hardware were custom built for the purpose of this project. Examples are the voltage-controlled power supply for the heating elements (see appendix A) and the $10-\mu \mathrm{A}$ constant current source for the excitation of the Lakeshore diode temperature sensor (see appendix B for the schematic diagram). A dedicated Labview software was written for the purpose of controlling the power supply output and monitoring the temperature of the stator assembly. 


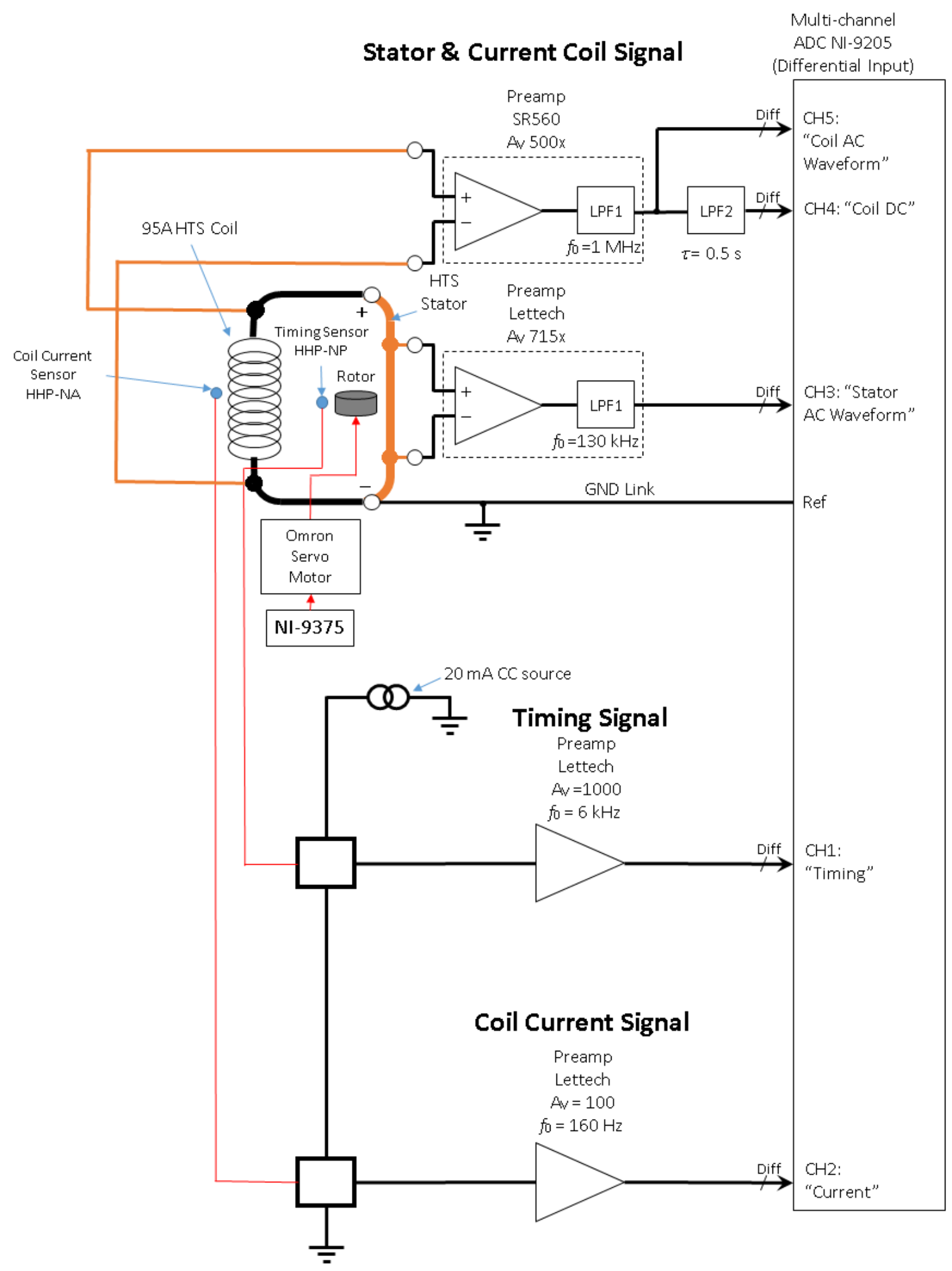

Figure 5.16: Gen 4 FP System Schematic and Signal Acquisition. CC: Constant current source, LPF: Low pass filter, Diff: Differential voltage input, $\mathrm{A}_{\mathrm{V}}$ : Voltage gain, $f_{0}$ : cut-off frequency, $\tau$ : Time constant. Modified from the original diagram supplied by A. E. Pantoja, RRI.

Unlike the Gen 2 system, there are two sets of voltage measurements on the Gen 4 
system. For the first one, a voltage signal across the stator is picked up via a set of voltage taps (figure 5.17a). This signal is amplified and sent to the NI acquisition unit. However, due to the geometry of the stator, the actual location to measure the stator voltage is difficult to determine. For this reason, another set of voltage taps is present on the HTS coil current leads (figure 5.17b). This measures the voltage across the coil, which is also in parallel with the FP stator, and the signal is amplified by the SR570 low-noise pre-amplifier before it is fed to the acquisition unit. However, note that the voltage across the coil $V_{\text {coil }}$ is equal to the FP voltage $V_{F P}$ minus the voltage drops on the contact resistances $I R_{c}$ between the flylead and the stator. This is described by the equation $V_{\text {coil }}=V_{F P}-I R_{c}$.

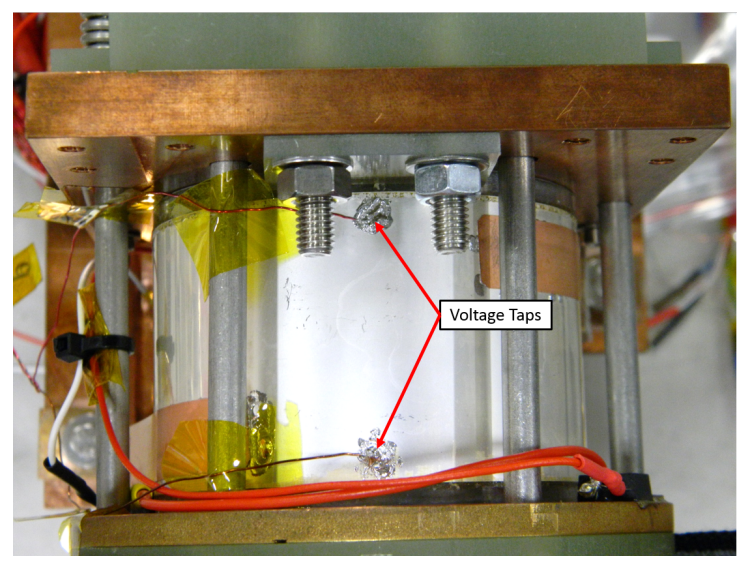

(a) Stator voltage taps

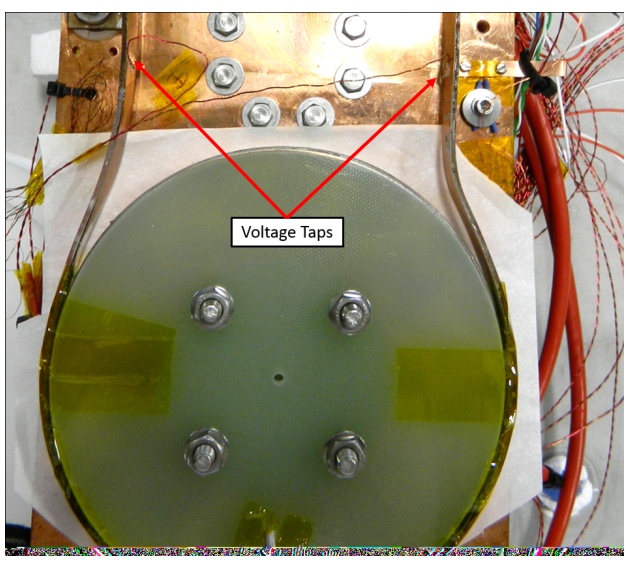

(b) Coil voltage taps

Figure 5.17: G4 FP system voltage taps.

The position of the magnet on the rotor with respect to the stator is detected by the HHNP Hall sensor. For every cycle of the rotor rotation, the voltage signal output of the Hall sensor varies according to the magnetic field strength. This signal is fed to a pre-amplifier and a LPF (noise reduction) before it is acquired by the NI-9205 [27].

The HTS current coil and the HTS stator are connected in series and share the same current. A calibrated Hall sensor is located at the centre of the coil to detect the current in the circuit. See section 5.1.3 for the calibration experiment.

In order to eliminate the error introduced by the lead resistance, 4-wire measurement method was used for the sensors signals. Differential input method was used for the voltage measurements at the acquisition card[28].

\subsection{Cooling Experiments}

In this section, initial cooling experiments performed on the Gen 4 flux pump system are discussed. Throughout the process, a few modifications and improvements were carried out on the test rig in order to improve the cooling performance. The test results will be presented in the chronological order which they were performed. 


\subsubsection{Initial Cooling Tests and PT100 Sensors Calibration}

Figure 5.18 shows two units of the calibrated PT100 and Lakeshore DT-670 temperature sensors installed on the cooling plate. The DT-670 sensors were installed in close proximity to the PT100 sensors so that the data could be used to calibration the PT100 sensors. The PT100 sensors were labelled as PT100\#1 and PT100\#2 and their temperature as T3 and T4 respectively. The DT-670 sensors were labelled as LK-D1 and LK-D2 and their temperature as T5 and T6 respectively. Prior to the calibration the standard calibration curve of the PT100 sensor was used to obtain the temperature readings.

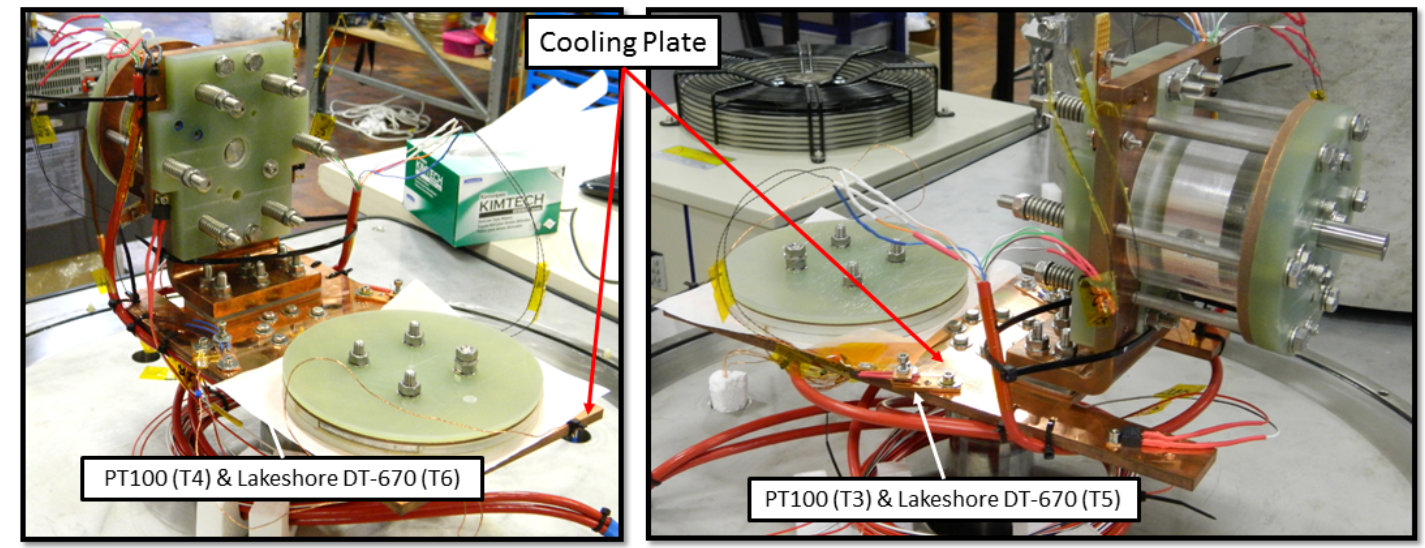

Figure 5.18: PT100 and Lakeshore DT-670 diode temperature sensors mounted on the cooling plate.

The result of the cooling test is shown in figure 5.19. The data of T5 and T6 show that the cooling plate reached $35 \mathrm{~K}$ within roughly 5 hours and the readings of the PT100 sensors readings were very close to each other. Since T5 and T6 were almost identical, T6 data was used to calibrate the PT100 sensors. The new calibration curves, shown in figure 5.20, were then used to convert the sensors resistance values to temperature for future experiments. The dotted sections are the extrapolated data using a second order polynomial function. 


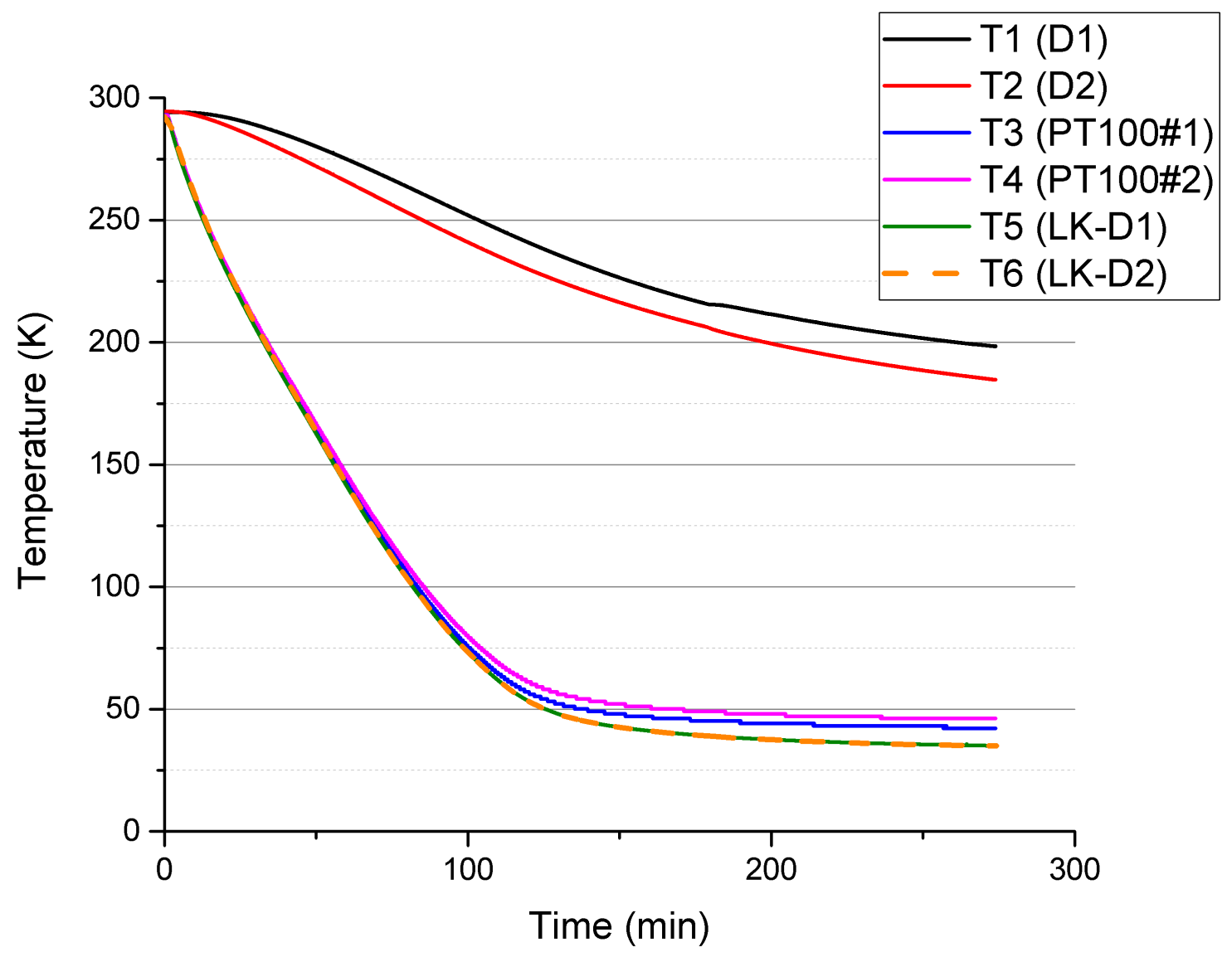

Figure 5.19: Gen 4 system cooling test. Additional DT-670 sensors were used to collect temperature data for the purpose of calibrating the PT100 sensors. 


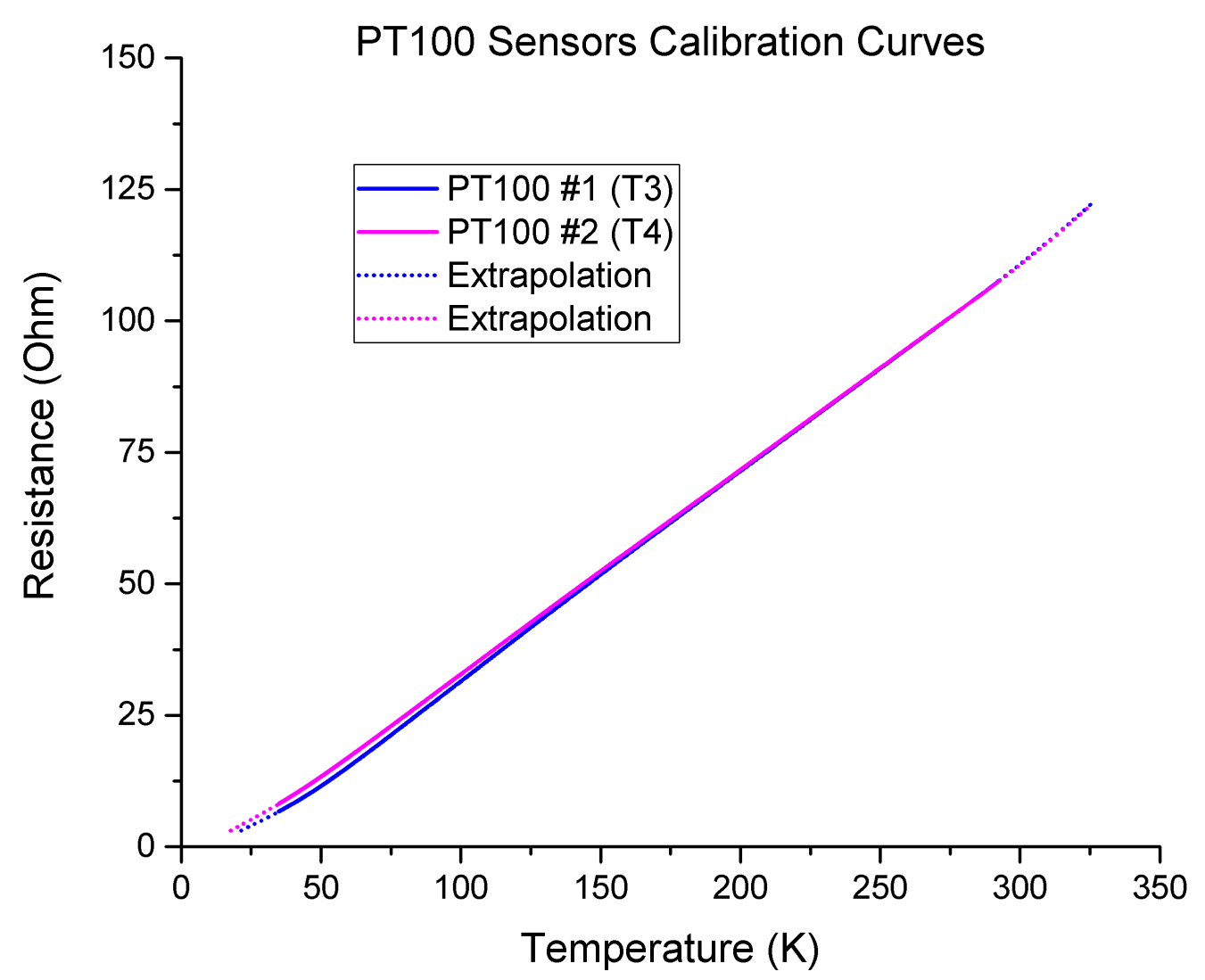

Figure 5.20: Gen 4 system PT100 sensors calibration.

Figure 5.19 shows that the temperature difference between the stator copper clamping plates and the cooling plate was about $155 \mathrm{~K}$. The reason is that a piece of G10 thermal break was present at the interface between the bottom clamping plate and the cooling plate. Prior to the experiment, it had been assumed that the stator assembly would be too cold and the heating elements would struggle to bring the stator to the desired temperature. However, the result shows that the stator was not cold enough, even after 5 hours of cooling. Therefore, the G10 thermal break was replaced by a copper piece for future experiments.

\subsubsection{Removal of the Thermal Break}

Figure 5.21 shows the test result after the G10 thermal break had been removed and replaced by a copper piece that had the same dimensions. A thin layer of Apiezon $\mathrm{N}$ grease was appplied on the interfaces between the copper piece, the cooling plate and the bottom copper clamping plate. 


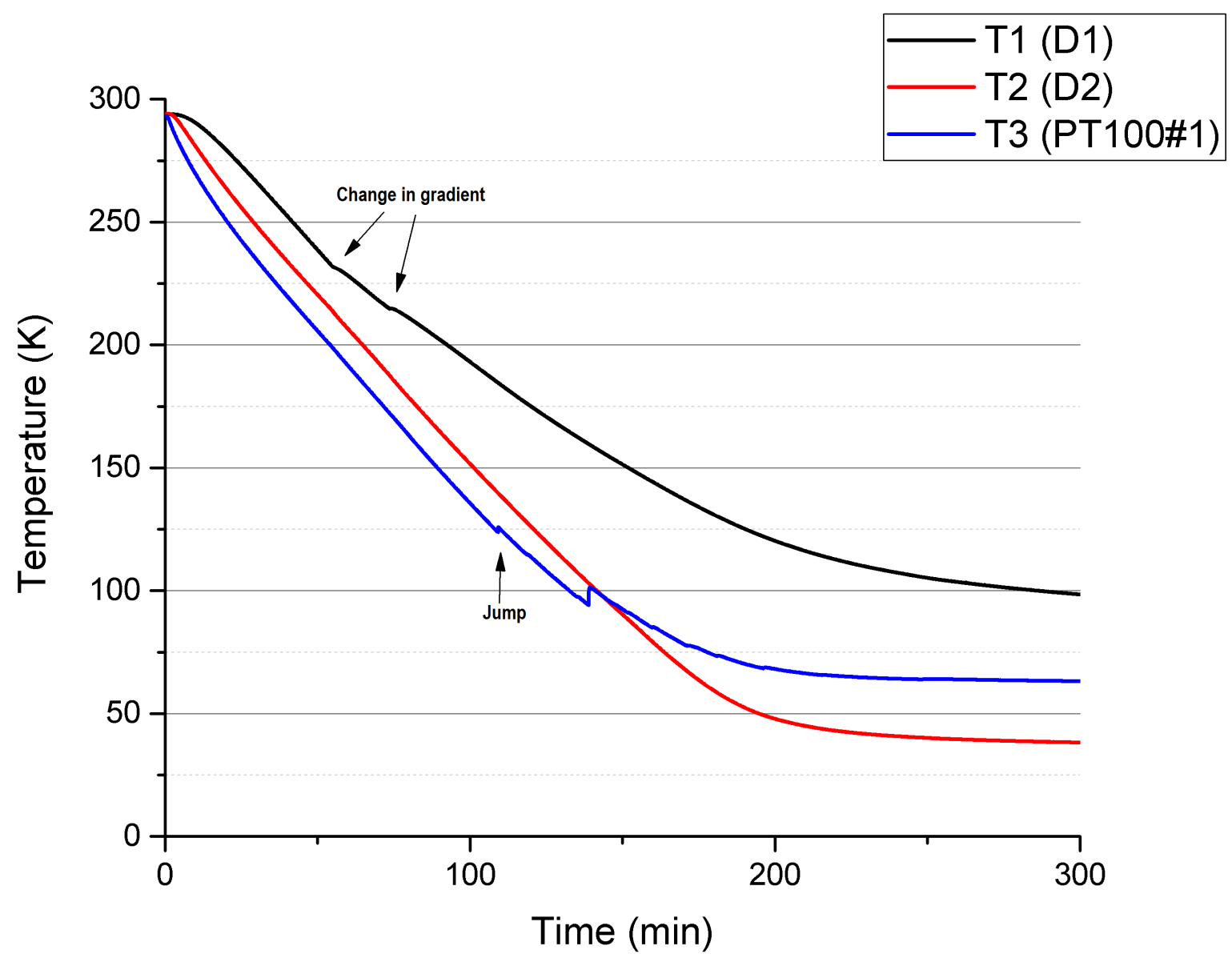

Figure 5.21: Gen 4 system cooling test after the G10 thermal break was replaced by a copper piece.

The data show that after about 5 hours, the lowest temperature (T2) measured on the bottom clamping plate was $39 \mathrm{~K}$. This is a substaintial improvement when compared with the previous measurement $(185 \mathrm{~K})$. However, the temperature (T1) of the top clamping plate was still considerably higher (98 K@ $300 \mathrm{~min}$ ). The reason is that the only thermal conduction path between the bottom clamping plate and the top clamping plate was the sapphire tube. There were several interfaces between the top clamping plate and cooling plate, each of these served as a thermal resistance. On top of that, the surface of the vacuum chamber also acted as a source of thermal radiation load.

Note that only one PT100 sensor (T3) was used in the measurement of the cooling plate. When the chamber was opened up for inspection it was noticed that the PT100 sensor came off from its mounting. It is assumed that this caused the sudden jump on the temperature curve at time about 109 minutes and 140 minutes. From this point onward, T3 was unreliable. The mounting problem was later solved and it did not occur in future experiments.

Also note that at 55 minutes and 73 minutes T1 curve shows a slight change in the gradient. However, it was later confirmed that the sensor D1 did not come off from the mounting and these changes in the gradient were probably due to thermal contraction movements at 
the interfaces between the sapphire tube and the clamping plates.

\subsubsection{Thermal Radiation Shield}

The previous experiments showed that the existing cooling arrangement was insufficient to overcome the thermal radiation load at the top clamping plate. In order to mitigate this problem, a thermal radiation shield was built to enclose the stator assembly. This is shown in figure 5.22. It was built from a multilayer insulation (MLI) held by a copper wire frame. The frame was attached to the bottom cooling plate so that it could reach the lowest temperature possible.

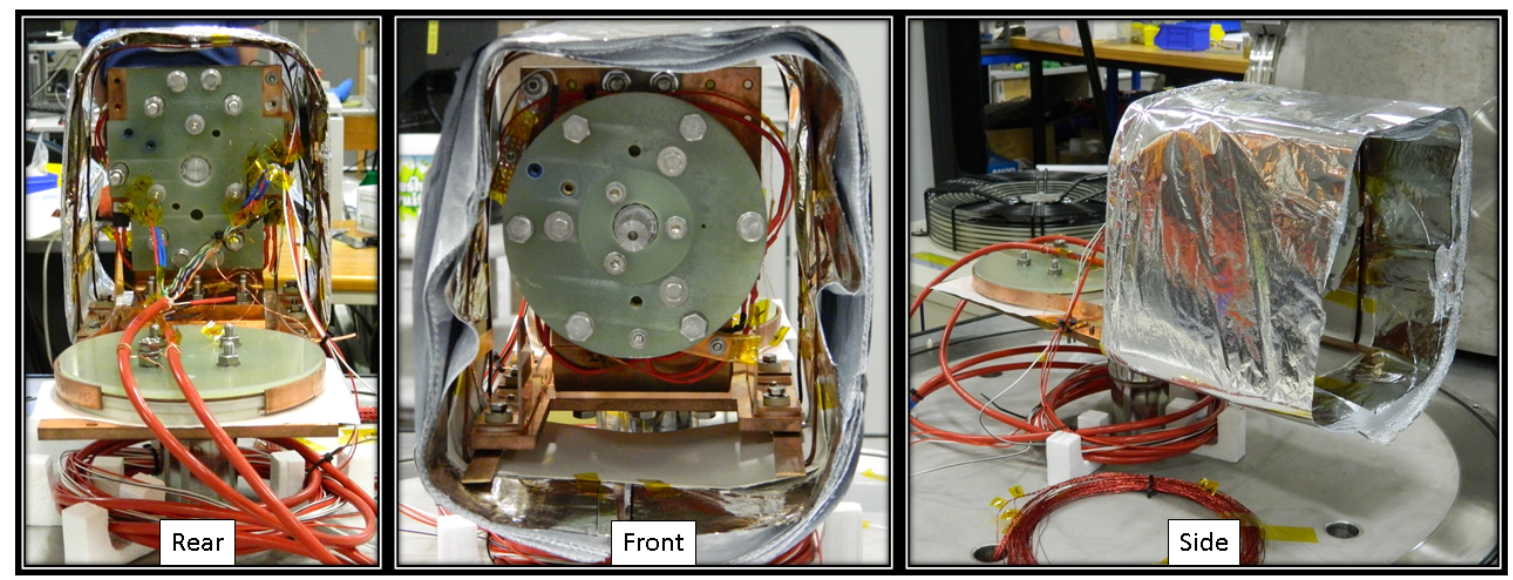

Figure 5.22: Gen 4 system stator assembly thermal radiation shield.

Figure 5.23 shows the cooling test after the installation of the radiation shield. At 400 minutes the temperature (T1) of the top clamping plate reached $75 \mathrm{~K}$, which was below the liquid nitrogen temperature. The bottom clamping plate (T2) was $32 \mathrm{~K}$ and the cooling plate (T3) was $22 \mathrm{~K}$. The result shows that the radiation shield reduced the heat load of the system significantly. 


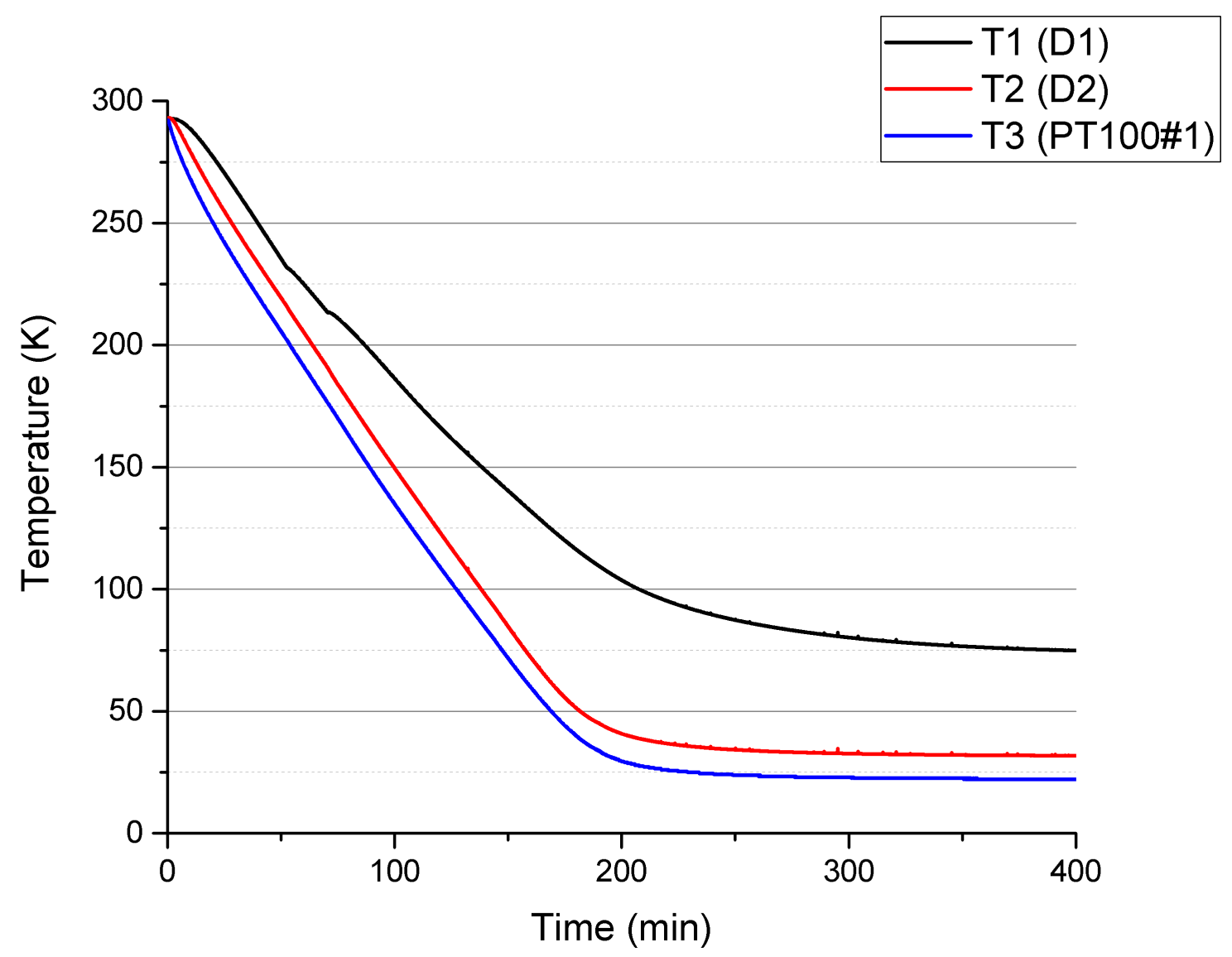

Figure 5.23: Gen 4 system cooling test after the installation of a radiation shield on the stator assembly.

\subsubsection{Thermal Bus}

The results from the previous experiments show that the radiation shield improved the cooling of the system. However, it was still insufficient to bring T1 lower than $75 \mathrm{~K}$. This was due to the thermal resistance in between the cooling plate and the top clamping plate. When the FP is in operation, the temperature difference between $\mathrm{T} 1$ and $\mathrm{T} 2$ is expected to increase due to heat generation in the stator. In order to overcome the temperature difference between the stator assembly and the cooling plate, a copper thermal bus was installed between the the top copper clamping plate and the cooling plate. This is shown in figure 5.24. The complete experimental setup, after the installation of the the radiation shield is shown in figure 5.25 . 


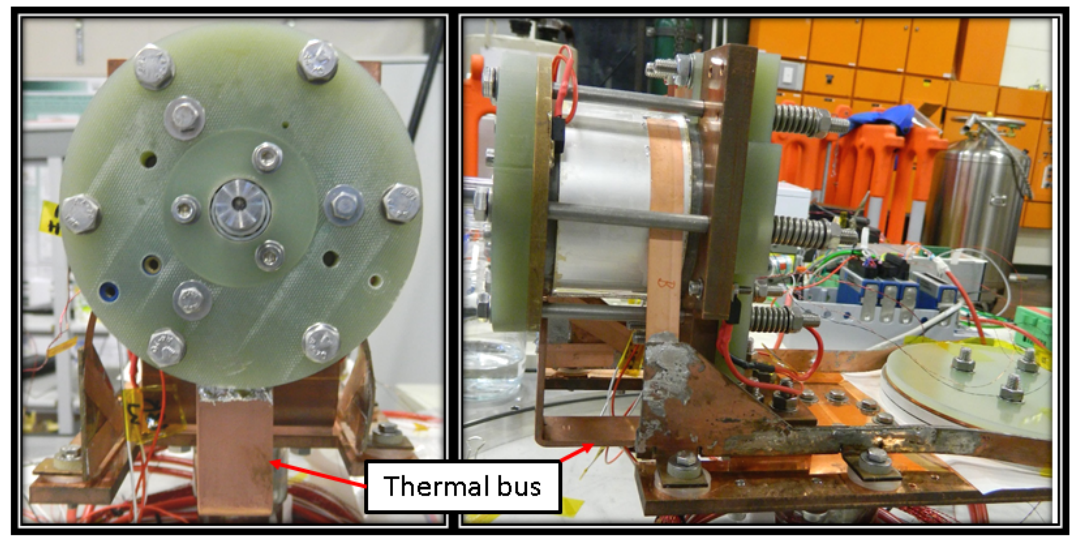

Figure 5.24: Gen 4 system - a thermal bus between the top clamping plate and the cooling plate.

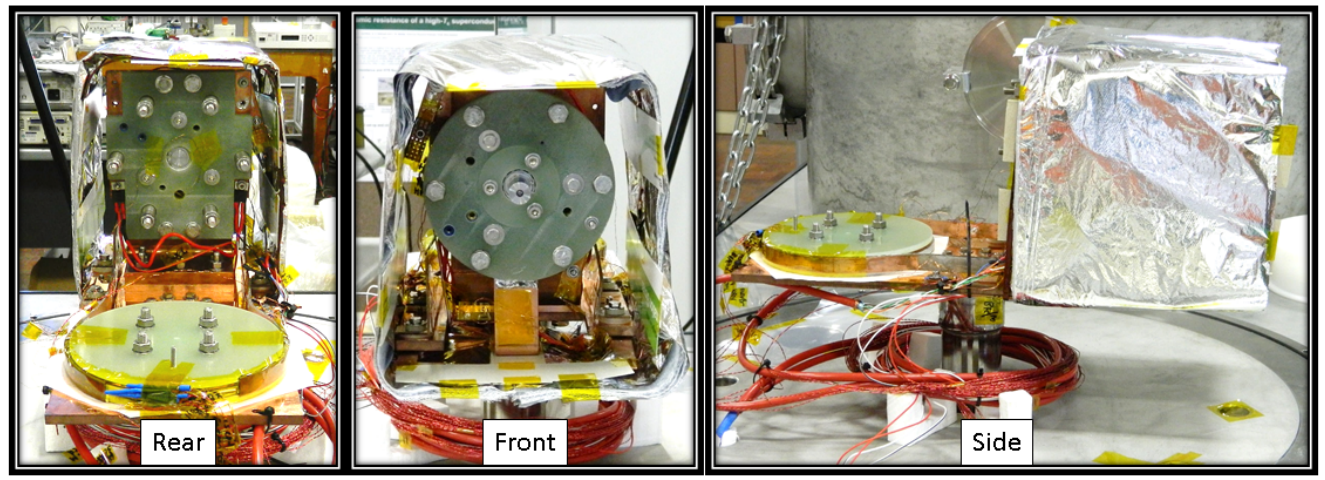

(a) Rear, front and side views of the G4 FP system with thermal bus and radiation shield.

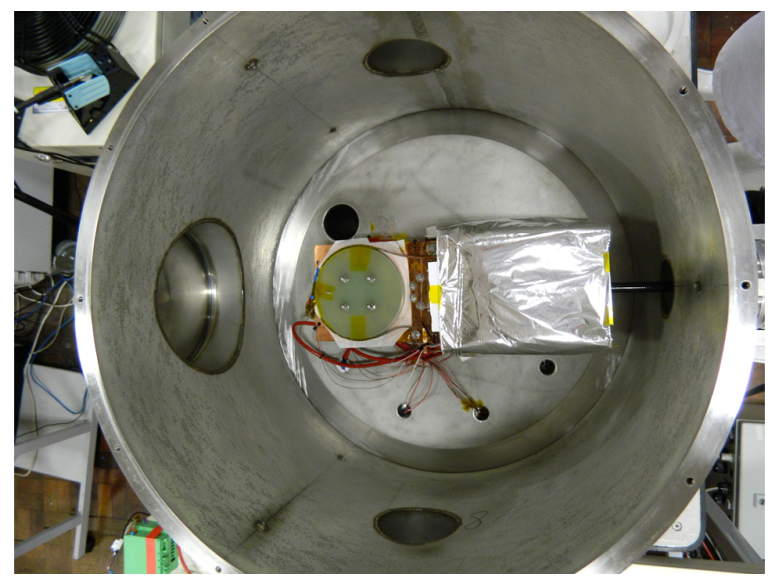

(b) Top view showing the rig located inside the vacuum chamber with the lid open.

Figure 5.25: Gen 4 system experiment final setup.

The cooling test result after the installation of the thermal bus is shown in figure 5.26. This time the temperature difference between the top clamping $\mathrm{T} 1$ and the bottom $\mathrm{T} 2$ was reduced significantly to just $4 \mathrm{~K}$. The temperatures $\mathrm{T} 1=37 \mathrm{~K}$ and $\mathrm{T} 2=33 \mathrm{~K}$ were achieved in about 250 minutes, which is faster compared with the previous run without the thermal bus. The final temperature of the cooling plate was $24 \mathrm{~K}$. The temperature level achieved at the stator assembly means that the temperature can be adjusted to the desired level (30 to 
$80 \mathrm{~K}$ ) using the heating elements. The results of the temperature dependent FP performance experiments will be discussed in chapter 6 .

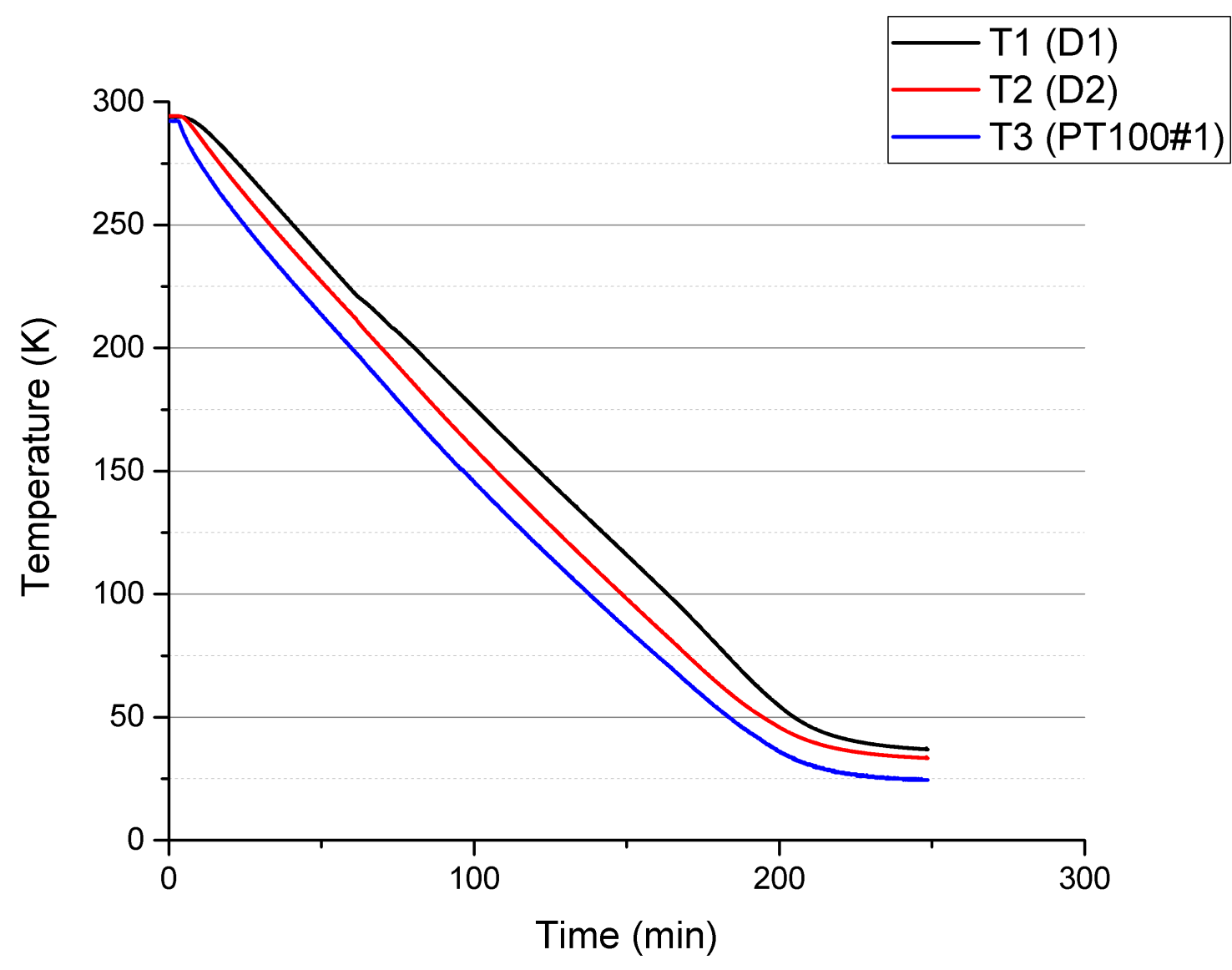

Figure 5.26: Gen 4 system cooling test after the installation of a thermal bus between the stator assembly and the cooling plate. 


\section{Chapter 6}

\section{Gen 4 FP System Experimental Results}

In this chapter, the experimental results for the Gen 4 FP system (see Chapter 5 for the system design) are discussed. The target stator operating temperatures in the experiment were 50,60, 70 and $80 \mathrm{~K}$. However, it was discovered that the exact stator temperature was difficult to achieve due to the self heating of the FP system and lack of cooling power from the cryo-cooler. Therefore, a method was employed in the experiment to stabilise the stator temperature, and this is explained. Then, an example of the AC waveform is analysed in order to investigate the mechanism that is responsible for the FP DC output. After that, the contact resistances in the FP circuit, $I-V$ curves and FP performance measured at various operating frequencies and temperatures are discussed. Finally, the frequency normalised results are compared with the results for the Gen 1 and Gen 2 FP systems and the temperature dependent characteristic of the Gen 4 system are discussed. 


\subsection{Temperature Monitoring}

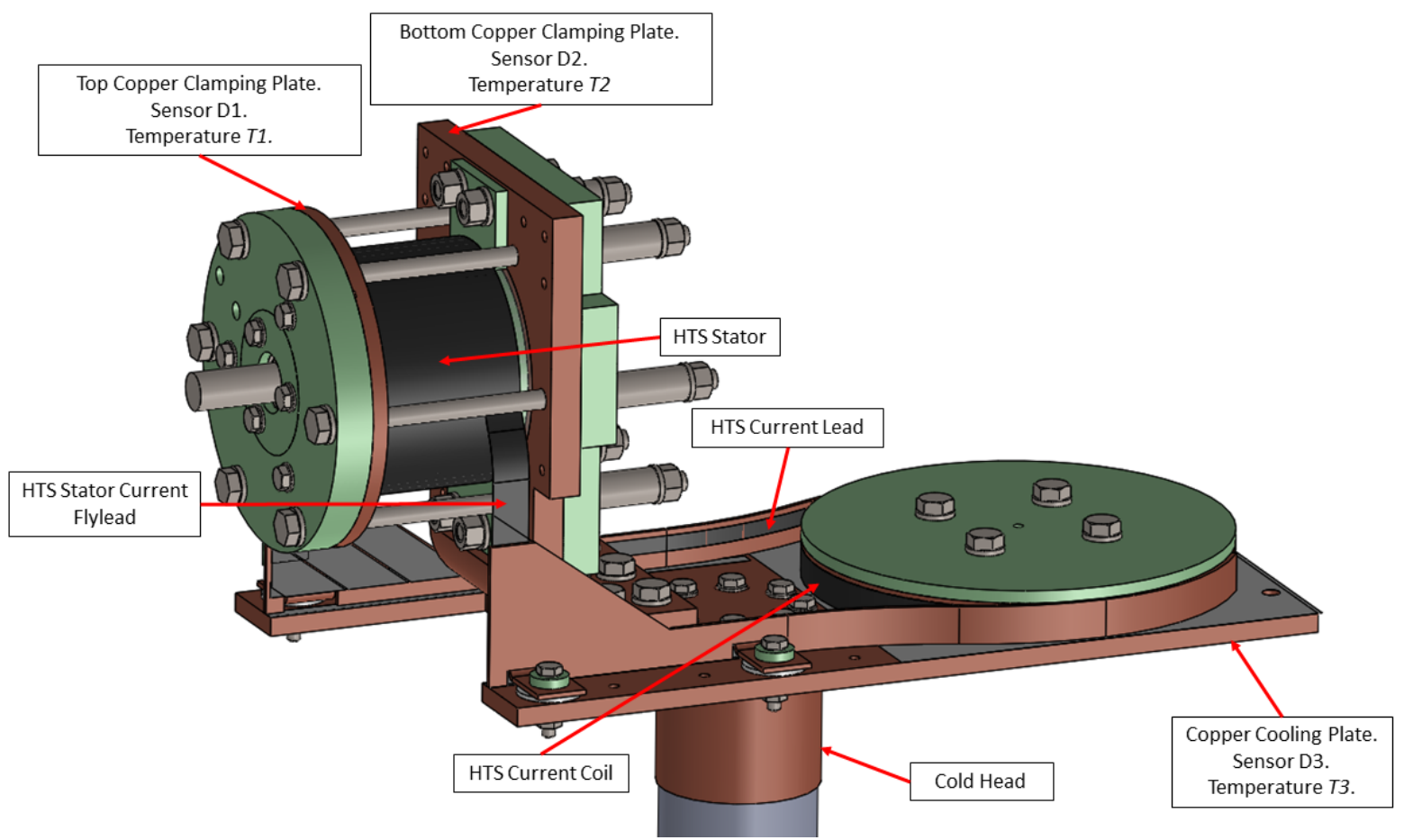

Figure 6.1: Temperature sensor locations of the Gen 4 FP system. RRI Solidworks model.

Figure 6.1 shows the locations of three temperatures sensors in the Gen 4 FP system. The first sensor, D1 (temperature T1) was attached to the top copper clamping plate. The second sensor, D2 (T2), monitored the temperature of the bottom clamping plate. The average temperature of these two sensors was recorded as the temperature of the stator $\left(T_{\text {avg }}\right)$. The difference between these two temperatures is denoted as $\Delta T(T 1-T 2)$. T3 denotes the temperature of the cooling copper plate. The stator generated heat during operation and this resulted in the change in $T_{\text {avg }}$ and $\Delta T$ over time, therefore, the maximum values of $T_{\text {avg }}$ and $\Delta T$ are presented in the results. However, the changes in $T_{\text {avg }}$ and $\Delta T$ over time were $\leq 2 \mathrm{~K}$.

\subsection{Temperature Stability}

In chapter 4, it was observed that the Gen 2 FP system appears to suffer heat quenching at high frequency operations due to the heat generated in the stators. It was found that the stator of the Gen 4 FP system also generated substantial amount of heat and this resulted in a difference in the temperature between the top and the bottom copper clamping plates. On top of that, the thermal resistance between the cryo-cooler cold head and the top clamping plate was much higher compared to the bottom clamping plate. At high operating frequencies and low operating temperature, the temperature difference $\Delta T$ between these plates was as large as $17 \mathrm{~K}$ because there was less cooling power from the cold head. This imposed a technical challenge in obtaining a stable operating temperature of the stator. 
A method has been employed in the experiment to achieve a stable operating temperature. This is shown in figure 6.2. First, $T 2$ was controlled (by setting the power supplied to the heating elements) until it reached the desired temperature. Then, the FP was turned on to supply current to the coil. In this current ramping period the stator was left to run and generate heat until $T 1$ and $T 2$ were stable. The FP was then quickly switched to the opposite operating direction (reverse) and the data was taken. Similarly, in the end of the reverse run, the FP was quickly switched to the forward direction for measurement. In the process of the measurements the heating elements on the top plate were turned off and a small power adjustment on the heating elements on the bottom plates was performed to compensate for the stator self heating. This method allowed for the temperature change in time to be kept relatively small. In general, the change in the average temperature with respect to time in the experiment was $\leq 2 \mathrm{~K}$.

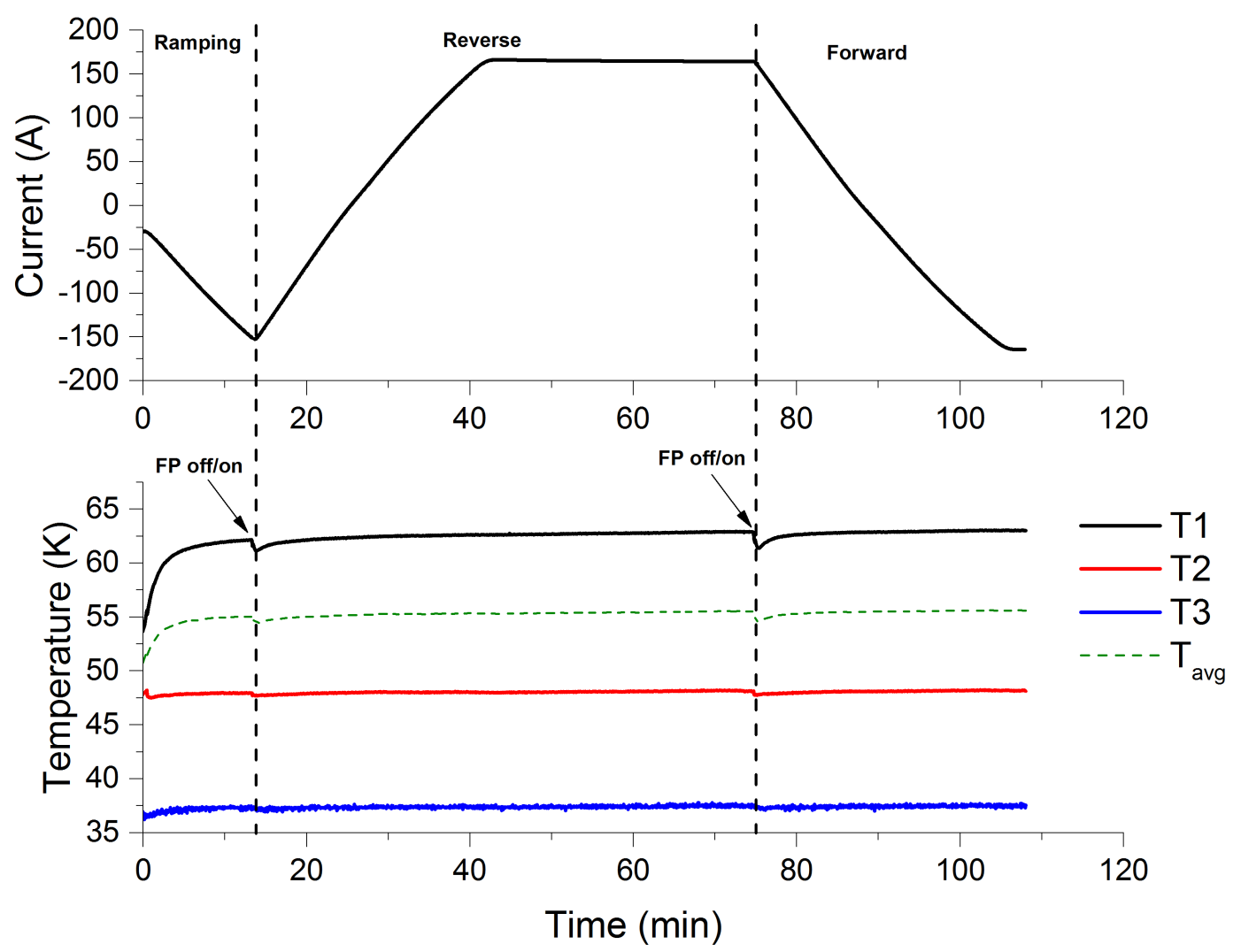

Figure 6.2: Achieving temperature stability. Current ramping, forward and reverse runs at $18 \mathrm{~Hz}, T_{\text {avg }}=55 \mathrm{~K}$.

\subsection{The AC Waveforms and the Open-Circuit Voltage Generation}

The Gen 4 HTS stator was made of the same superconducting material as used in the Gen 2 system. Therefore, the waveform analysis used for the Gen 2 system can be employed here to investigate the non-zero average voltage generated by the Gen 4 FP stator.

As discussed in chapter 5 , the stator was made of a $46 \mathrm{~mm}$ wide HTS tape rolled into a 
cylinder shape. Due to the geometry of the stator, it was difficult to identify well defined points at which the voltage across the stator could be measured. For this reason, another set of voltage taps was used to measure the voltage developed across the current coil (see figure $5.17 \mathrm{~b}$ ). Figure 6.3a shows the superconducting AC waveform (black line) of the voltage measured across the coil at $25 \mathrm{~Hz}$ at $80 \mathrm{~K}$. The yellow dotted line (labelled "normal") is the $\mathrm{AC}$ waveform measured at the same frequency when the FP was in normal conducting state. This was measured when the cooling plate temperature had reached $100 \mathrm{~K}$. At this cooling plate temperature stator temperature was assumed to be well above the YBCO critical temperature $(93 \mathrm{~K})$. The red dot line is the average DC voltage $(80 \mathrm{~K}, 25 \mathrm{~Hz})$ measured separately to the AC waveform, using a low-pass DC filter as discussed in chapter 5. A hall sensor (see figure 5.4a) was installed in the stator assembly to detected the magnetic field of the rotor magnet. The minimum of the timing signal (blue line) indicates the moment when the magnet was located at the lowest position of the rotor angle, which was defined as $0^{\circ}$. Note that the actual amplitude of the timing signal is irrelevant and therefore it is not plotted to scale.

The $\mathrm{AC}$ waveforms measured at $80 \mathrm{~K}$ and at the normal conducting state are very different in shape. It can be seen that rectification occurred such that $V_{o c}>0$ for almost the entire rotor cycle. In comparison, the waveforms in the Gen 2 system were only partially rectified and this occurred only when the magnets were crossing over the stator (e.g. see figure 4.25). Figure $6.3 \mathrm{~b}$ shows the integration of the waveforms over a full rotor cycle. For the $80 \mathrm{~K}$ waveform the final integral value is about $0.05 \mathrm{mV} / \mathrm{Hz}$, which is consistent with the measured DC value. For the normal conducting waveform, the final value of the integration is zero.

The high degree of rectification in the Gen 4 system is not entirely surprising, because the rotor magnet was always located opposite the stator. Therefore, it is reasonable to expect that the stator produces DC output voltage throughout the rotor cycle. This shows that the Gen 4 FP system is far more effective than the Gen 1 and Gen 2 FP systems in terms of producing DC voltages. 


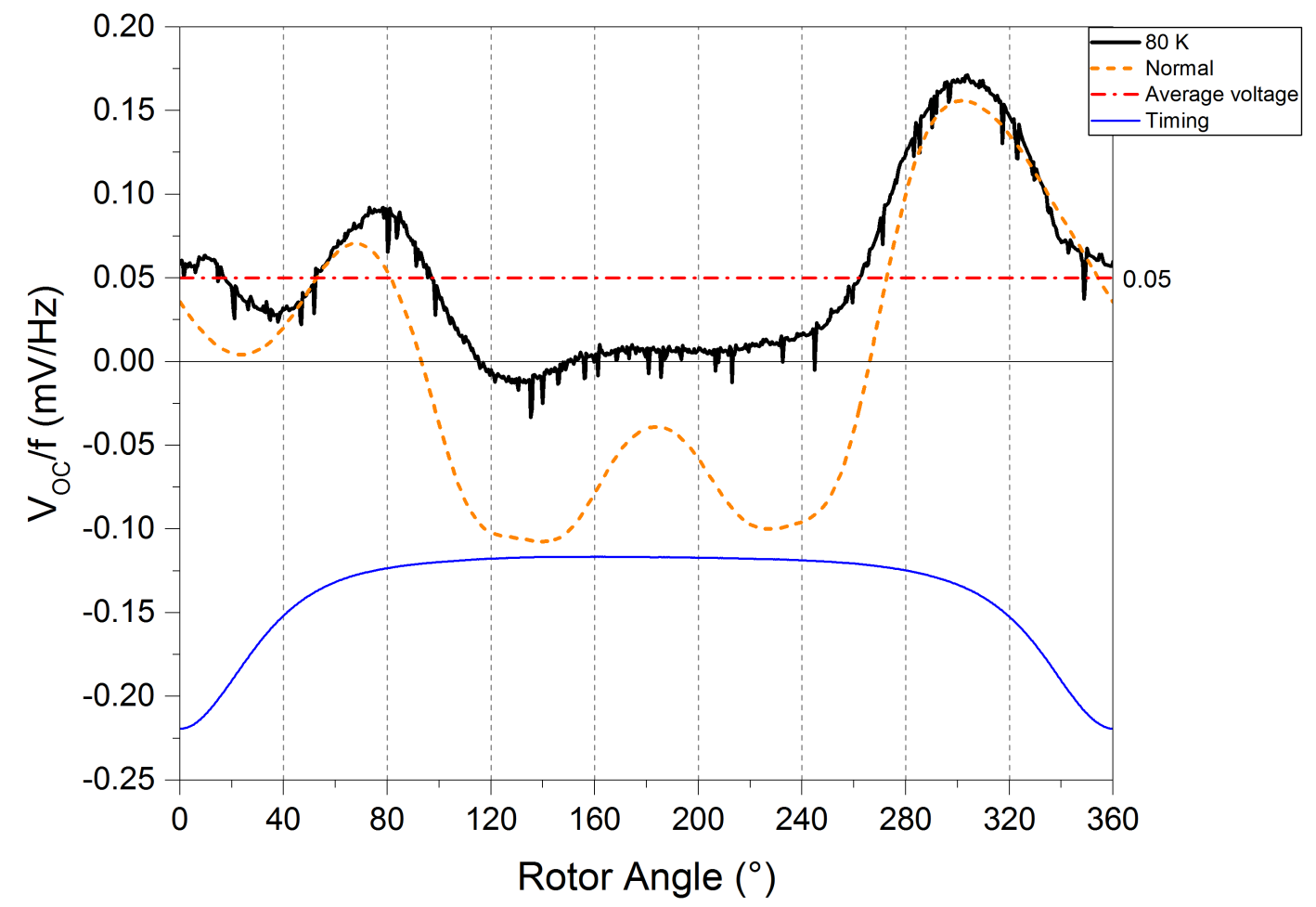

(a) AC waveforms measured at superconducting $(80 \mathrm{~K})$ and normal conducting states. The timing signal is not plotted to scale. The downward spikes occurred on the $80 \mathrm{~K}$ waveforms are believed to be due to the noise interference coupled to the signal leads. The actual source of the noise is unknown, although it appeared related to the operation of the variable frequency inverter used to control the servo motor in this experiment.

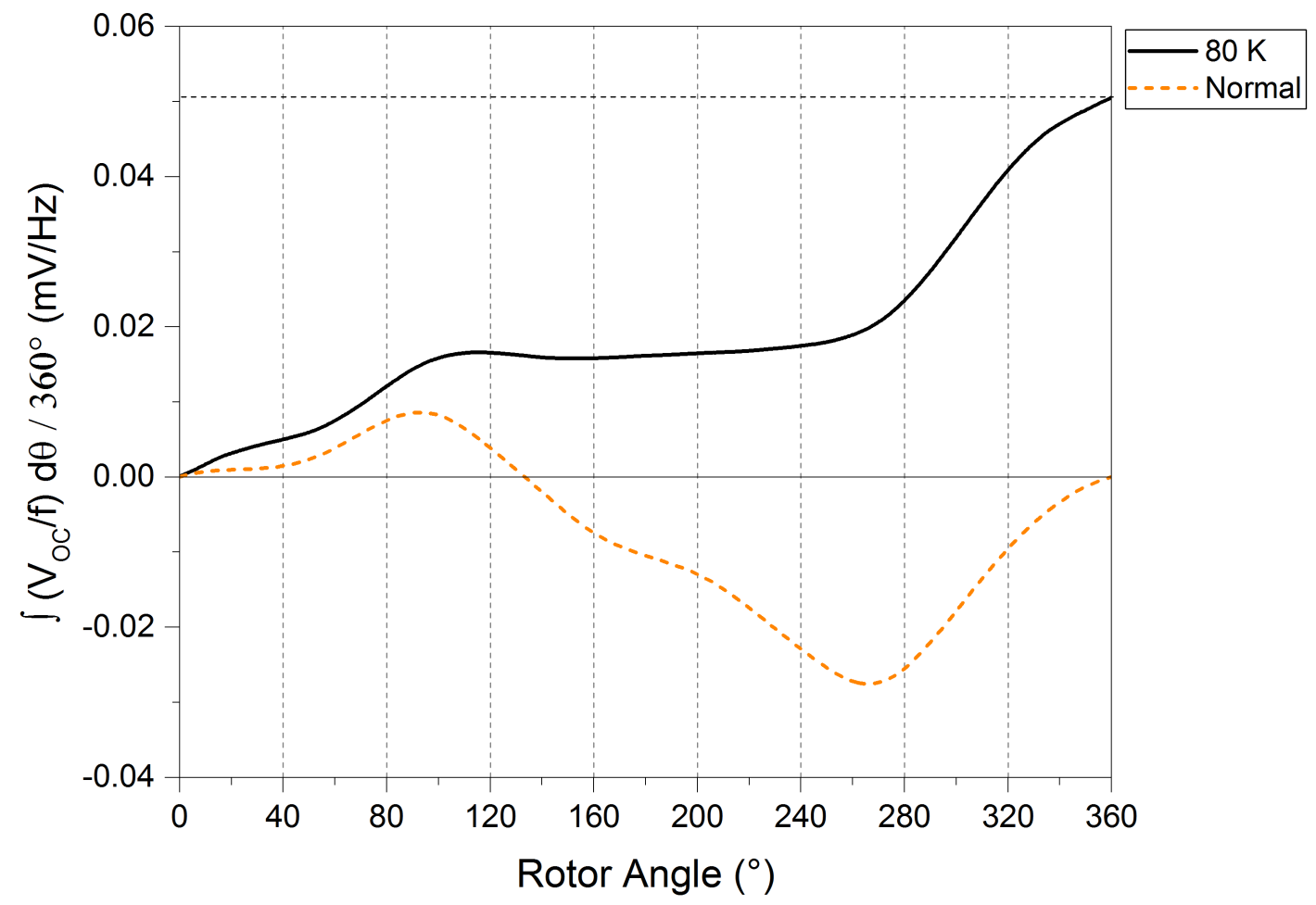

(b) AC waveform integration over a full rotor cycle.

Figure 6.3: Frequency-normalised open-circuit voltage waveform measured at $25 \mathrm{~Hz}$ at 80 K. 


\subsection{The DC Equivalent Circuit, Contact Resistances and Flux Pump Voltage}

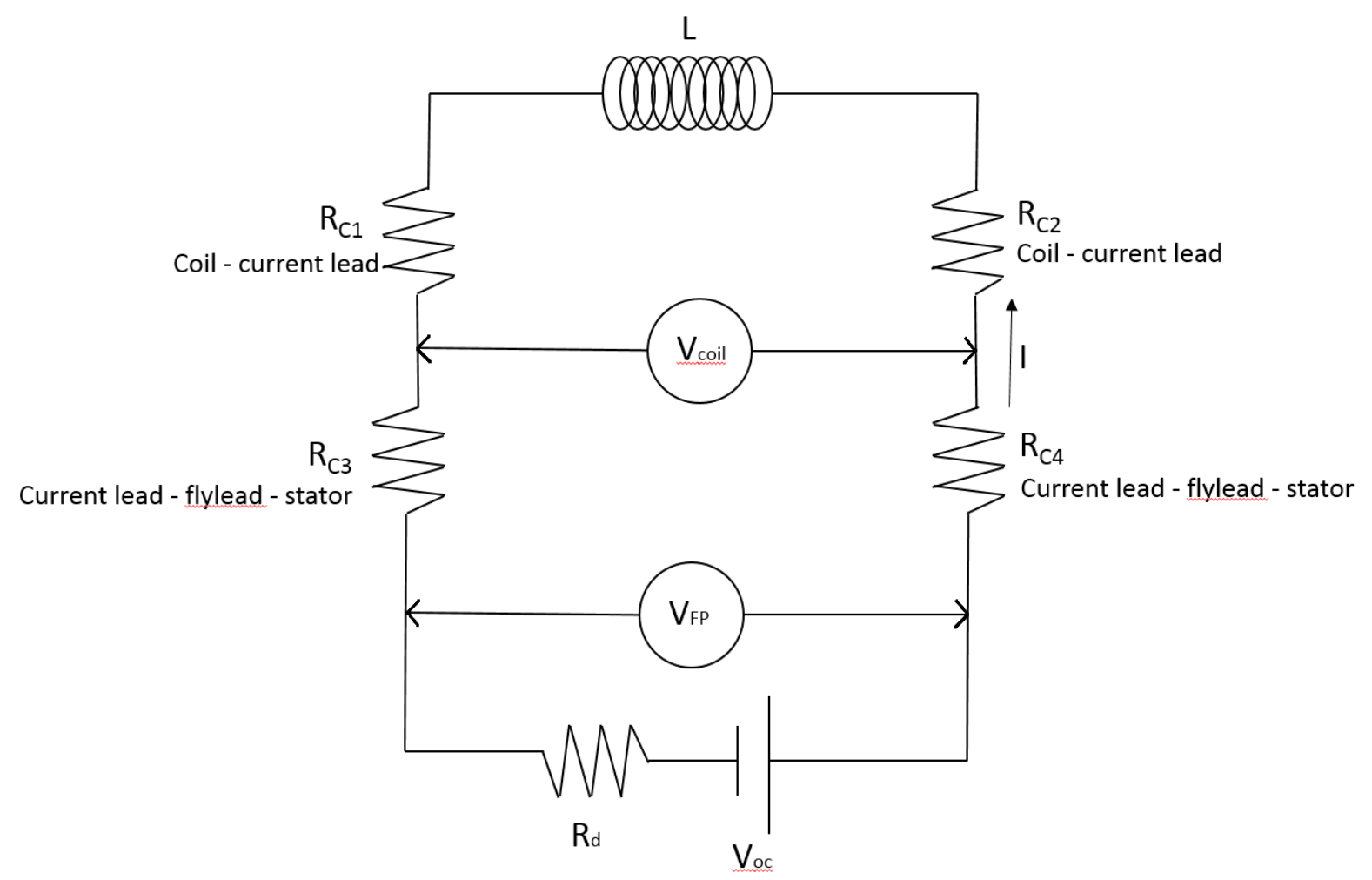

Figure 6.4: Gen 4 FP DC equivalent circuit.

The DC behaviour of the Gen 4 system is expected to be similar to the Gen 1 and Gen 2 systems. An equivalent DC model for the Gen 4 system is shown in 6.4. Similar to the Gen 2 system, the Gen 4 stator was connected in series with a current coil. However, the Gen 4 system has more connections: between the current leads and the coil; between the current leads and the flyleads; and between the flyleads and the stator (see chapter 5, section 5.1.2.3 for the photos). $R_{d}$ represents the dynamic resistance of the FP and $V_{o c}$ represents the opencircuit voltage (measured across the stator at open-circuit condition). At zero-load (e.g. if the superconducting coil is ideal and there are no contact resistances), the short-circuit current $I_{s c}$ of the FP is:

$$
I_{s c}=\frac{V_{o c}}{R_{d}}
$$

The coils in the Gen 2 and Gen 4 systems were manufactured to the same specifications. In order to determine the value of the total contact resistance in the Gen 4 FP circuit, current was initially injected into the coil using the FP. The FP was then turned off to allow for the current to decay. A log-linear line was fitted to the decay current data and the time constant was calculated from the slope of the line. The total contact resistance in the FP was then 
calculated from the time constant. This method was identical to the method discussed in chapter 4 , section 4.2 .3 for Gen 2 system.

Table 6.1 shows a summary of the contact resistances at different temperatures of the cooling plate.

\begin{tabular}{|c|c|}
\hline Cooling plate temperature $(\mathrm{K})$ & Contact resistance $R_{C T}(\mu \Omega)$ \\
\hline \hline 35 & 0.24 \\
\hline 45 & 0.26 \\
\hline 55 & 0.29 \\
\hline 62 & 0.31 \\
\hline
\end{tabular}

Table 6.1: FP circuit total contact resistance.

As mentioned earlier, the voltage $\left(V_{\text {coil }}\right)$ developed across the coil was measured in the experiment. The actual FP voltage $\left(V_{F P}\right)$ can be calculated from:

$$
V_{F P}=V_{c o i l}+I R_{C}^{\prime}
$$

where $R_{C}^{\prime}=R_{C 3}+R_{C 4}$ and $I$ is the current in the FP circuit. However, only the total contact resistance is known, therefore, as an approximation, $V_{F P}$ is calculated from:

$$
V_{F P} \approx V_{\text {coil }}+I R_{C T}
$$

where $R_{C T}=R_{C 1}+R_{C 2}+R_{C 3}+R_{C 4}$ is the total contact resistance.

In order to estimate the error in $V_{F P}$ in equation 6.3 , the total resistance $R_{C}^{\prime \prime}=R_{C 1}+R_{C 2}$ has to be estimated. Each of the resistances $R_{C 3}$ and $R_{C 4}$ are the result of three contact joints. On the other hand, each of the resistances $R_{C 1}$ and $R_{C 2}$ are due to one contact join. Therefore, it is assumed that $R_{C}^{\prime \prime}=\frac{R_{C T}}{4}$. For the case of $R_{C T}=0.31 \mu \Omega$, at $170 \mathrm{~A}$ (approximately the maximum current obtained in the experiment) the voltage developed across $R_{C}^{\prime \prime}$ is about $0.01 \mathrm{mV}$. Therefore, for $V_{F P}>0.1 \mathrm{mV}$ the error in the measurements is estimated to be $<10 \%$.

\subsection{The FP Performance}

Gen 4 FP system is capable of producing larger DC output voltages compared with the Gen 1 and Gen 2 systems because the stator is much wider than the rotor magnet. This is consistent with the findings reported in [31] which showed that, for a fixed magnet size and operating frequency, $V_{o c}$ increased linearly with stator width until a maximum frequency is reached. The authors also reported that at low operating frequencies the frequencynormalised dynamic resistance $\left(R_{d} / f\right)$ of the stator reached a maximum value when the widths of the stator and magnet were about the same. For larger stator widths, the dynamic resistance decreased and this resulted in high $I_{s c}$. Therefore, the Gen $4 \mathrm{FP}$ is expected to produce small $R_{d}$ and large $I_{s c}$, provided that it operates at low enough frequency and is not thermally quenched. 


\subsubsection{Current vs Time Plot}

Figure 6.5 shows the FP current plotted with respect to time for the target temperature 50 $\mathrm{K}$. The rotor was rotating in the reverse direction for the three operating frequencies shown. $T_{\text {avg }}$ is the average temperature of the stator and $\Delta T$ is the difference in the temperature between the top and bottom clamping plate (see figure 6.1). The maximum current $\left(I_{\text {sat }}\right)$ produced by the FP was approximately $166 \mathrm{~A}$.

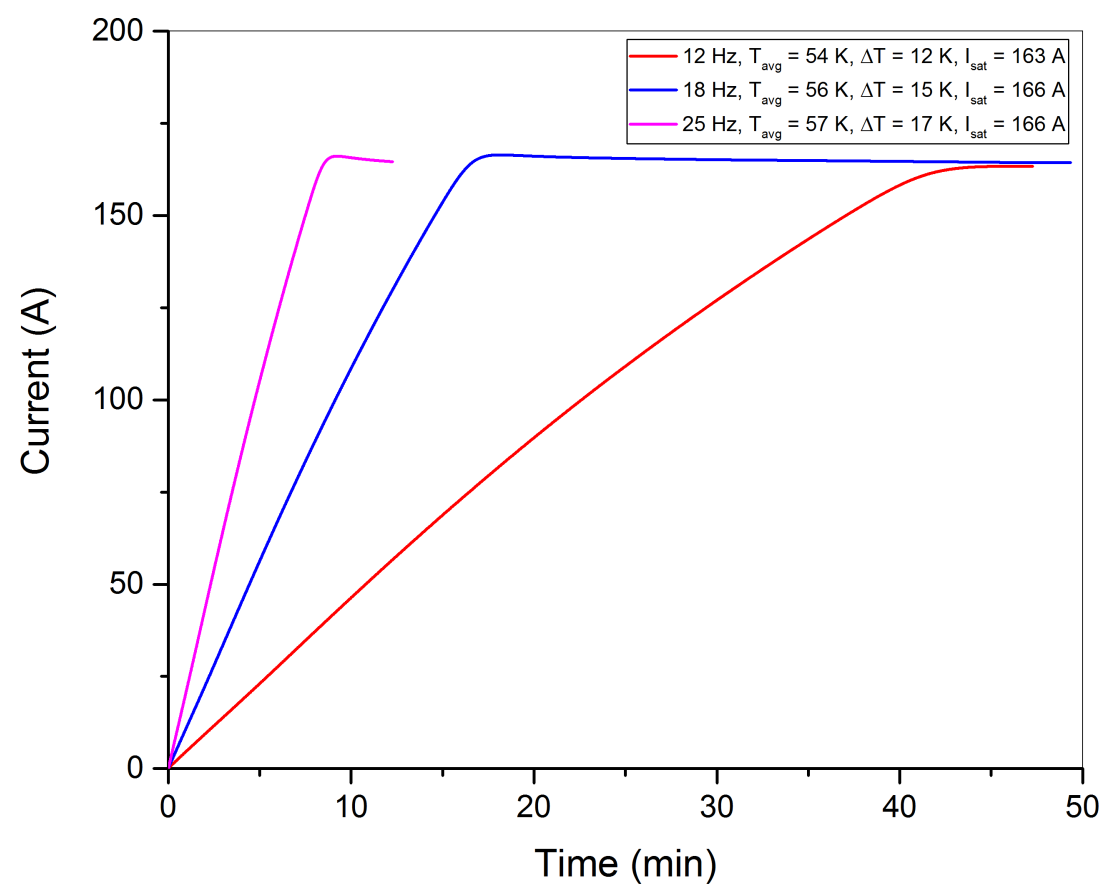

Figure 6.5: FP circuit current as a function of time for various operating frequencies at target temperature $50 \mathrm{~K}$.

\subsection{2 $I-V$ Curves}

Figure 6.6 shows the $I-V$ plots for the target temperature of $50 \mathrm{~K}$ for reverse and forward rotor directions. The reverse data set is the same as the the data set shown in figure 6.5.

For the reverse direction, the measurements started from negative coil current. As the motor was ramping up in speed, the FP voltage $V_{F P}$ was increasing in value until it reached thermally stable state (e.g. point A). From there onwards, the current and the voltage are assumed to follow a linear relationship.

The values for the open-circuit voltage $\left(V_{o c}\right)$, dynamic resistance $\left(R_{d}\right)$ and short-circuit current $I_{s c}$ can be obtained from the $I-V$ curves. This is done by fitting a linear line to the the data points in the driven current region as follows:

Reverse rotor direction $0 \leq I \leq I_{\text {sat }}$ and $V_{F P} \geq 0$

Forward rotor direction $0 \geq I \geq I_{\text {sat }}$ and $V_{F P} \leq 0$ 


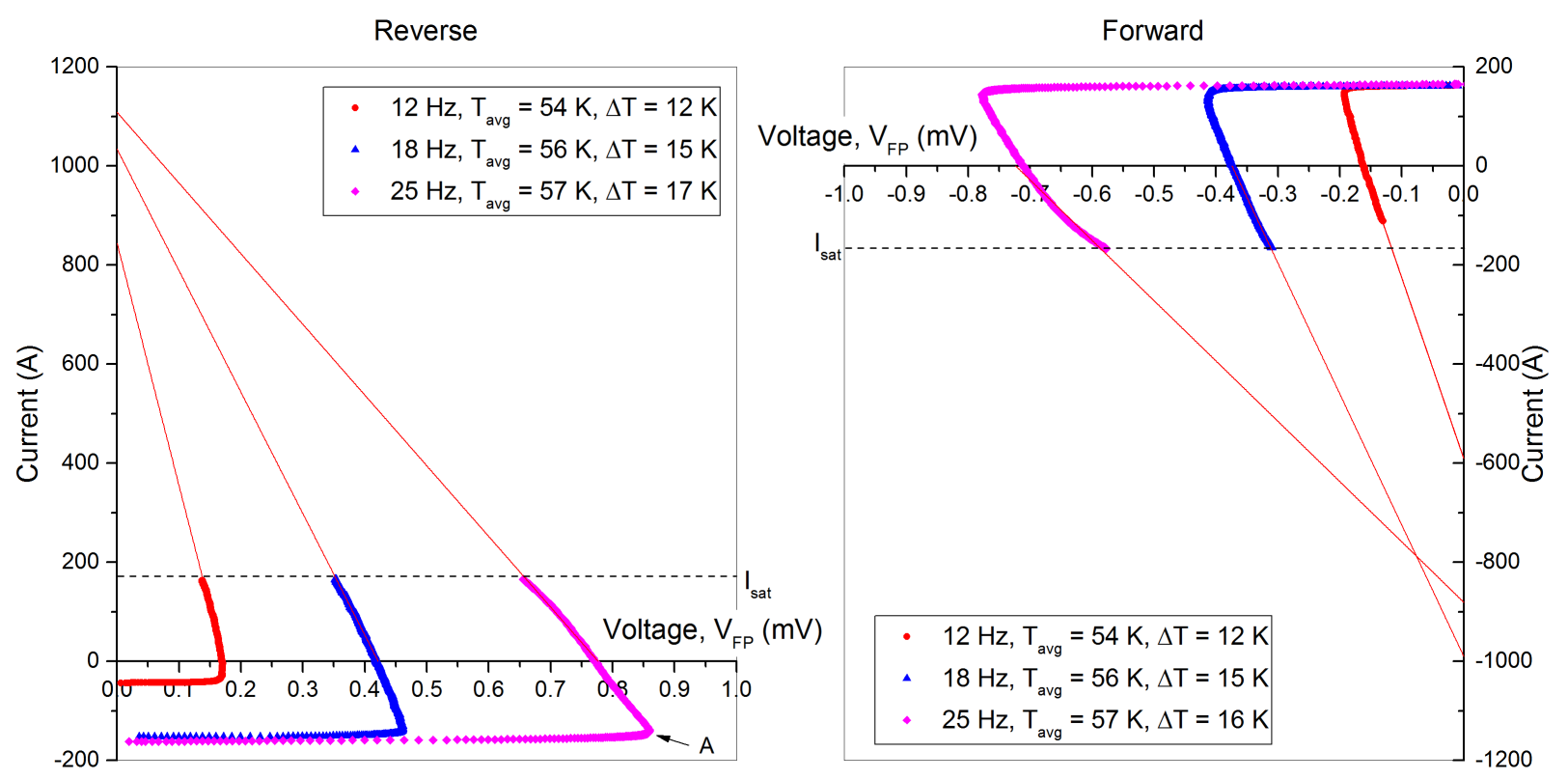

Figure 6.6: $I-V$ curves for three frequencies measured at target temperature $50 \mathrm{~K}$. The dotted lines represent the saturation current $I_{\text {sat }}$. The red lines are the line fits for the extrapolation of $I_{s c}$. The $12 \mathrm{~Hz}$ forward current saturated at a lower (absolute) value. One possibility is that the current leads were at higher temperature when the data was taken.

Note that the values of $I_{\text {sat }}$ are very small and are believed to be caused by damaged current leads. Also, the data used for the line fit is assumed to be linear based on the experimental results obtained on the Gen 1 and Gen 2 FP systems. However, due to the limited range of data, there is no evidence that the data beyond this range should follow the same trend. In fact, the high frequency data (e.g. $25 \mathrm{~Hz}$ in figure 6.6) shows some nonlinearity. For this reason, the value of $I_{s c}$ obtained via linear extrapolation must be treated as an estimation with high uncertainty. In contrast, the value of $V_{o c}$ has very small uncertainty because it was obtained from a direct measurement. $R_{d}$ by definition is obtained from the inverse of the slope of the $I-V$ curve. Since the range of data is limited, the possibility that $R_{d}$ is not constant for $|I|>\left|I_{\text {sat }}\right|$ cannot be ruled out.

It was observed that the $I-V$ curves were almost identically symmetric in the reverse and forward directions. Therefore, for the rest of this section only the reverse $I-V$ curves are discussed.

The $I-V$ curves for the other target temperatures are shown in figure 6.7. Here, only the data in the driven current region was plotted and used for line fit. Note that as the temperature increased, $I_{s a t}$ decreased. This suggests that the $I_{c}$ of the current leads and flyleads decreased as the temperature increased, which is expected for any HTS wires. 


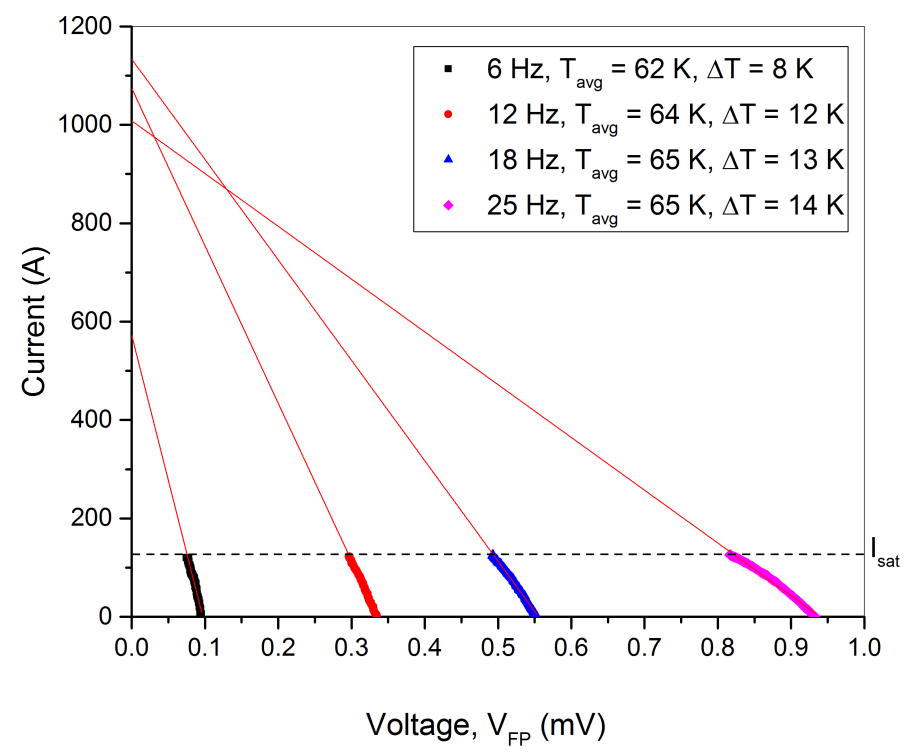

(a) Target temperature $60 \mathrm{~K}$

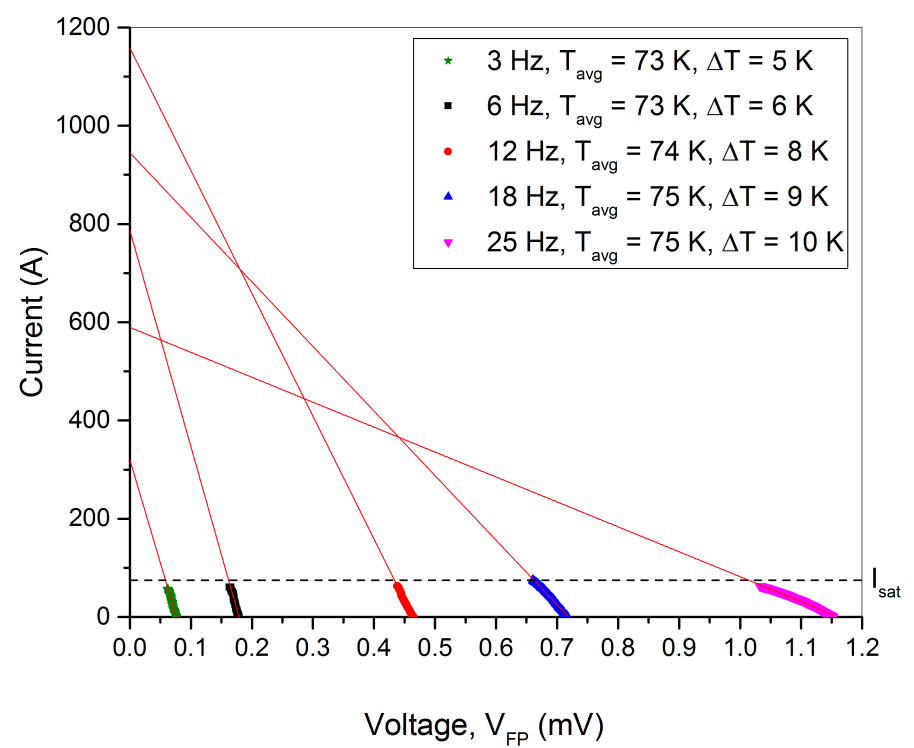

(b) Target temperature $70 \mathrm{~K}$

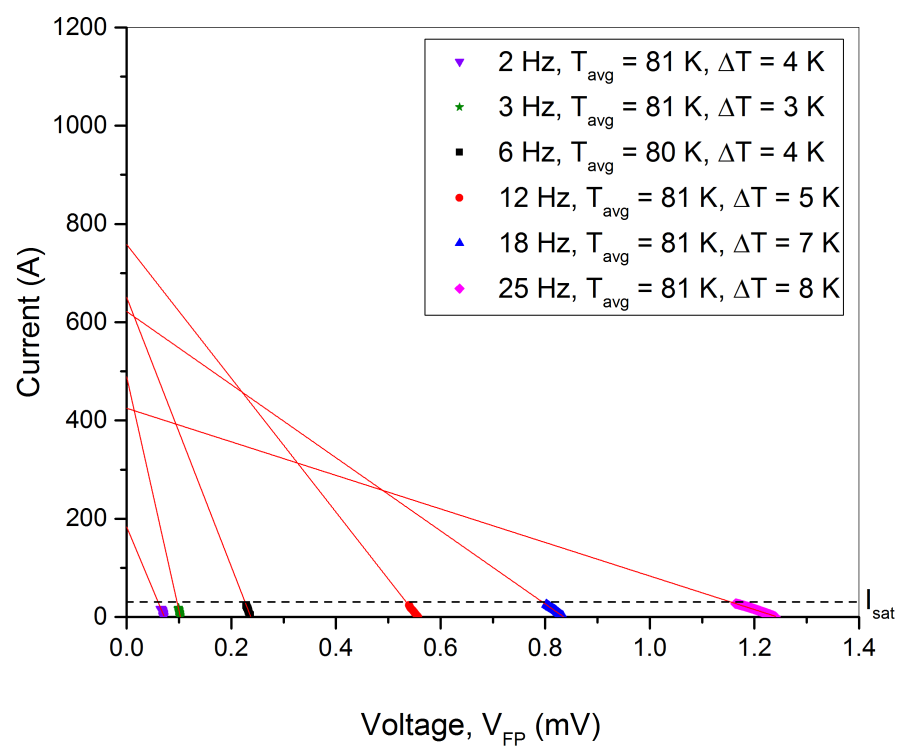

(c) Target temperature $80 \mathrm{~K}$

Figure 6.7: $I-V$ curves measured at target temperatures 60,70 and $80 \mathrm{~K}$, reverse direction. The dotted lines represent the saturation current $I_{\text {sat }}$. The red lines are the line fits. 


\subsubsection{Line fit error}

Since the line fits on the $I-V$ curves were done on a limited range of data, it is necessary to investigate the errors in the line fits. Tables 6.2 and 6.3 show the FP performance parameters extracted from the line fits for target temperature of $50 \mathrm{~K}$. The error of one $\sigma$ in each of the line fit is included in the table. It can be seen that the errors are very small. For example, for the reverse direction at $12 \mathrm{~Hz}$, the errors in $R_{d}$ and $I_{s c}$ are about $0.2 \%$ and $0.4 \%$ respectively.

\begin{tabular}{|c|c|c|c|c|c|c|}
\hline Frequency $(\mathrm{Hz})$ & $\mathrm{V}_{\mathrm{oc}}(\mathrm{mV})$ & $\sigma\left(V_{o c}\right)(\mathrm{mV})$ & $R_{d}(\mu \Omega)$ & $\sigma\left(R_{d}\right)(\mu \Omega)$ & $\mathrm{I}_{\mathrm{sc}}(\mathrm{A})$ & $\sigma\left(\mathrm{I}_{\mathrm{sc}}\right)(\mathrm{A})$ \\
\hline \hline 12 & 0.17257 & 0.00005 & 0.2043 & 0.0004 & 845 & 3 \\
\hline 18 & 0.42163 & 0.00006 & 0.4077 & 0.0006 & 1034 & 1 \\
\hline 25 & 0.77681 & 0.00016 & 0.7008 & 0.0015 & 1108 & 2 \\
\hline
\end{tabular}

Table 6.2: FP performance parameters measured at target temperature $50 \mathrm{~K}$, reverse direction. Linear line fitted to data $0 \leq I \leq I_{\text {sat }}$ and $V_{F P} \geq 0$.

\begin{tabular}{|c|c|c|c|c|c|c|}
\hline Frequency $(\mathrm{Hz})$ & $\mathrm{V}_{\mathrm{oc}}(\mathrm{mV})$ & $\sigma\left(V_{o c}\right)(\mathrm{mV})$ & $R_{d}(\mu \Omega)$ & $\sigma\left(R_{d}\right)(\mu \Omega)$ & $\mathrm{I}_{\mathrm{sc}}(\mathrm{A})$ & $\sigma\left(\mathrm{I}_{\mathrm{sc}}\right)(\mathrm{A})$ \\
\hline \hline 12 & 0.16181 & 0.00003 & 0.2742 & 0.0004 & 590 & 1 \\
\hline 18 & 0.37413 & 0.00006 & 0.3781 & 0.0006 & 990 & 1 \\
\hline 25 & 0.72326 & 0.00031 & 0.8213 & 0.0030 & 881 & 3 \\
\hline
\end{tabular}

Table 6.3: FP performance parameters measured at target temperature $50 \mathrm{~K}$, forward direction. Linear line fitted to data $0 \geq I \geq I_{\text {sat }}$ and $V_{F P} \leq 0$.

The errors in the line fits for the target temperature of $80 \mathrm{~K}$ is expected to be the highest compared with other target temperatures because it has the lowest $I_{\text {sat }}$. Table 6.4 shows the extracted FP performance parameters for the target temperature of $80 \mathrm{~K}$ in the reverse direction. It shows that the line fit errors in the $80 \mathrm{~K}$ data are higher than the $50 \mathrm{~K}$ data in tables 6.2 and 6.3, as expected. However, the percentage uncertainties are still very small. For example, at $2 \mathrm{~Hz}$, the errors in $I_{s c}$ and $R_{d}$ are about $2.7 \%$ and $3.1 \%$ respectively. For this reason, the line fit uncertainty in the experimental data are not included in the plots and will not be discussed any further. However, as pointed out earlier, due to the limited range of data for the line fits the uncertainty in the extrapolated value for $I_{s c}$ is high and the possibility that $R_{d}$ is not constant at $|I|>\left|I_{\text {sat }}\right|$ cannot be ruled out.

\begin{tabular}{|c|c|c|c|c|c|c|}
\hline Frequency $(\mathrm{Hz})$ & $\mathrm{V}_{\mathrm{oc}}(\mathrm{mV})$ & $\sigma\left(V_{o c}\right)(\mathrm{mV})$ & $R_{d}(\mu \Omega)$ & $\sigma\left(R_{d}\right)(\mu \Omega)$ & $\mathrm{I}_{\mathrm{sc}}(\mathrm{A})$ & $\sigma\left(\mathrm{I}_{\mathrm{sc}}\right)(\mathrm{A})$ \\
\hline \hline 2 & 0.07211 & 0.00014 & 0.393 & 0.012 & 184 & 5 \\
\hline 3 & 0.10274 & 0.00006 & 0.210 & 0.005 & 489 & 11 \\
\hline 6 & 0.23753 & 0.00005 & 0.365 & 0.004 & 651 & 6 \\
\hline 12 & 0.55707 & 0.00036 & 0.734 & 0.004 & 759 & 4 \\
\hline 18 & 0.83612 & 0.00009 & 1.344 & 0.005 & 622 & 2 \\
\hline 25 & 1.24470 & 0.00025 & 2.929 & 0.013 & 425 & 2 \\
\hline
\end{tabular}

Table 6.4: FP performance parameters measured at target temperature $80 \mathrm{~K}$, reverse direction. Linear line fitted to data $0 \leq I \leq I_{\text {sat }}$ and $V_{F P} \geq 0$. 


\subsubsection{FP Performance Parameters}

Figure 6.8 shows the plots for the FP performance parameters $\left(V_{o c}, R_{d}\right.$ and $\left.I_{s c}\right)$ vs frequency for the reverse and forward rotor directions. The $V_{o c}$ and $R_{d}$ in both directions are almost identical and this reflects the symmetry in the stator design. At first glance, the $V_{o c}$ plots do not appear to be linear. However, it is important to note that the data has high uncertainty in the average stator temperature $T_{\text {avg }}$ due to large $\Delta T$, especially at high operating frequencies. Similar, the non-linearity in $R_{d}$ could be due to high uncertainty in the temperature. 


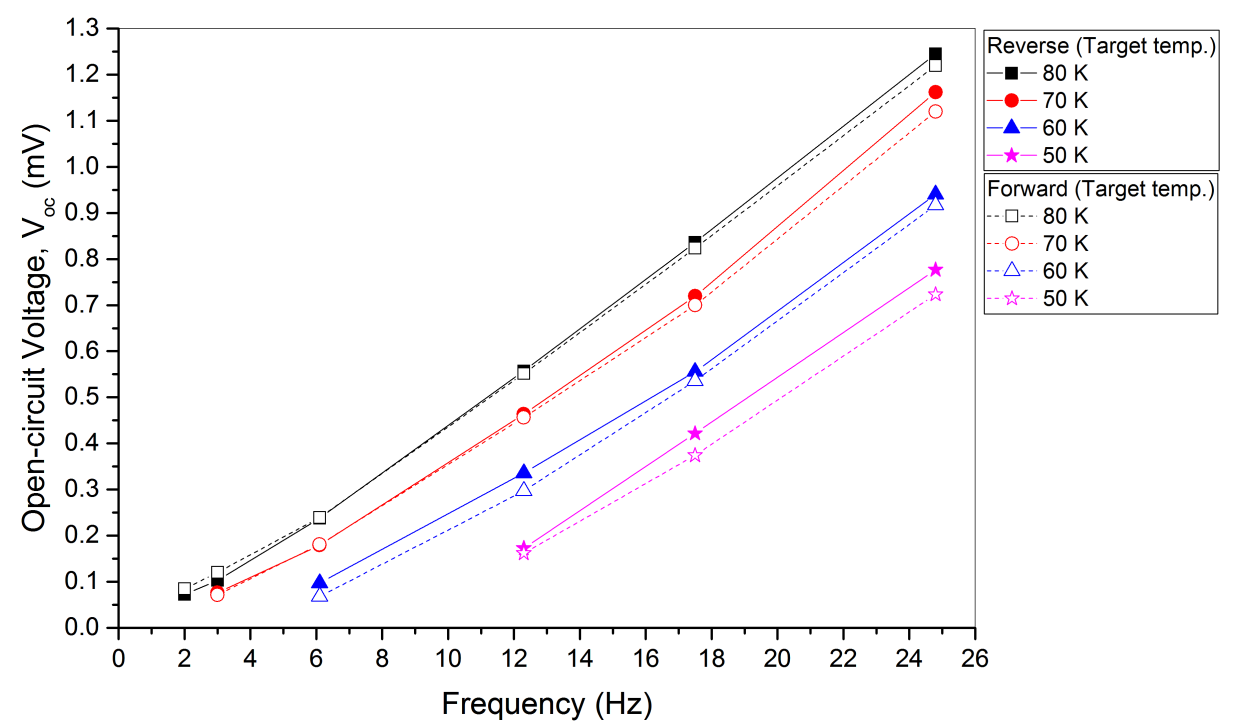

(a) Open-circuit voltage $\left(V_{o c}\right)$

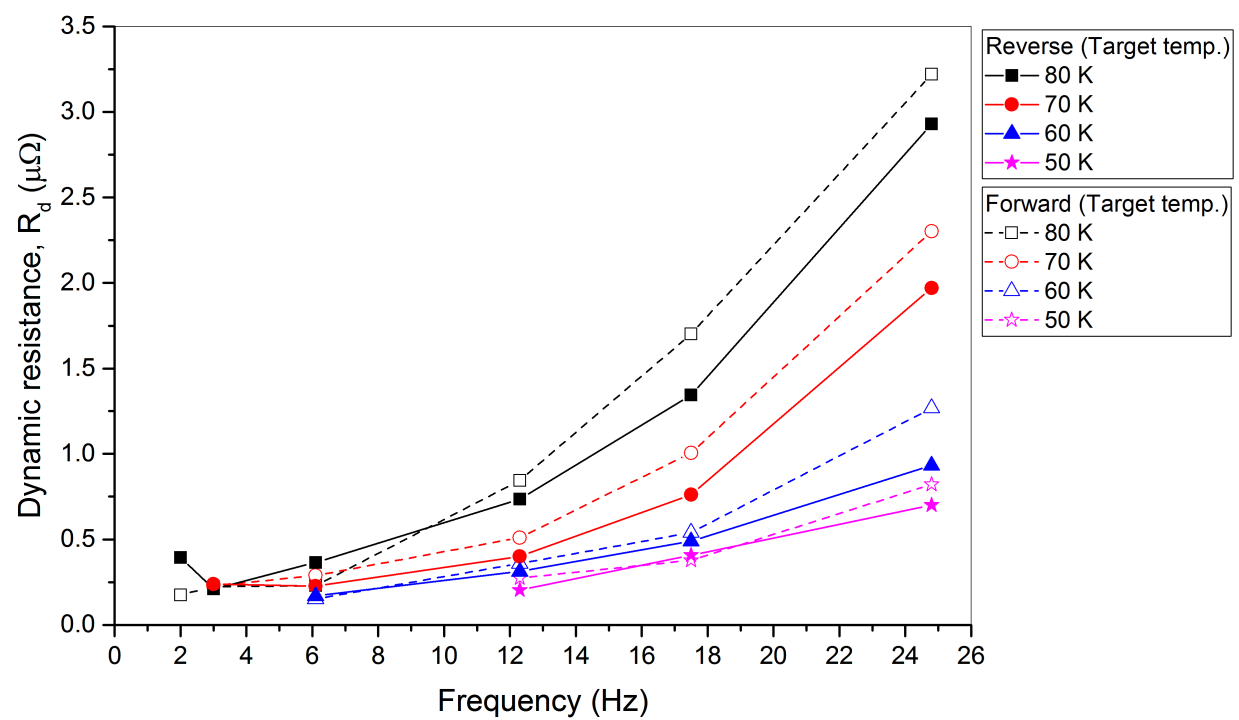

(b) Dynamic resistance $\left(R_{d}\right)$

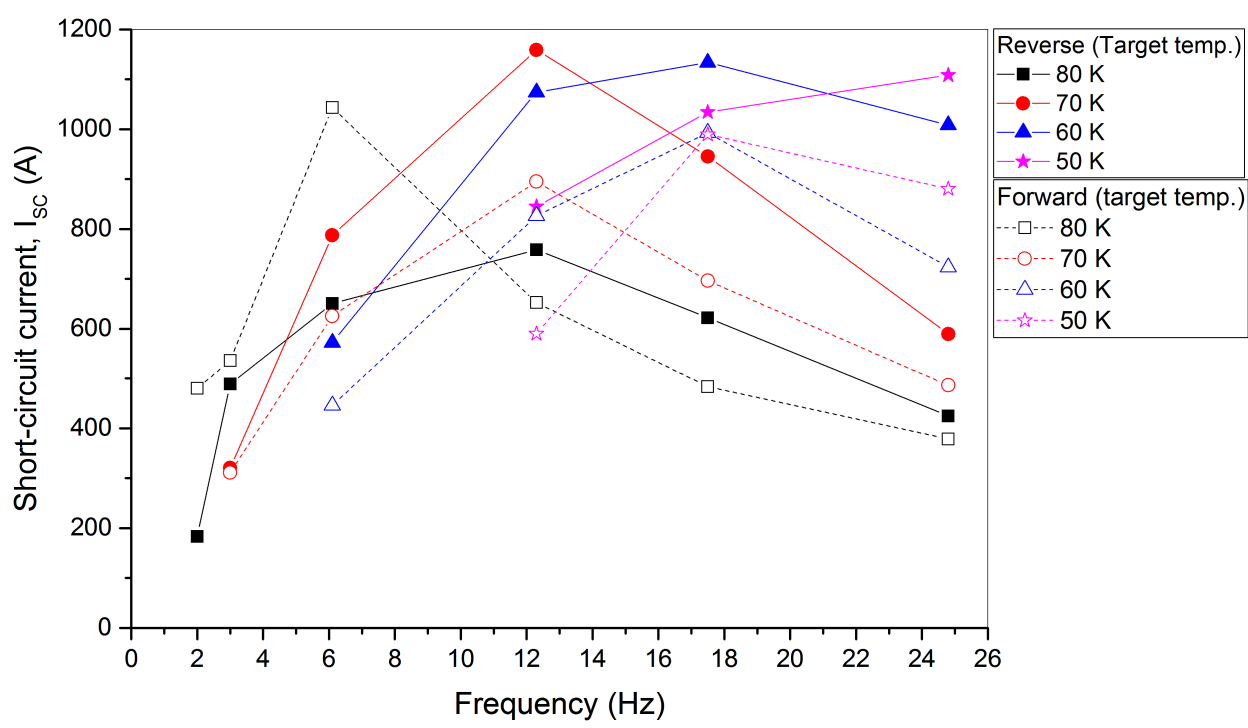

(c) Short-circuit current $\left(I_{s c}\right)$

Figure 6.8: FP performance as a function of frequency for all target temperatures. The lines joining the data points are guides to the eye. 
The plots for FP performance parameters $\left(V_{o c}, R_{d}\right.$ and $\left.I_{s c}\right)$ vs the average measured stator temperature $T_{a v g}$ are shown in figure 6.9. Again, $V_{o c}$ and $R_{d}$ in both directions are almost identical because the FP is axially symmetric. Although, as noted before, the uncertainty in the stator average temperature is high, the $V_{o c}$ plots nevertheless show a general trend of diminishing as the temperature gets lower. 


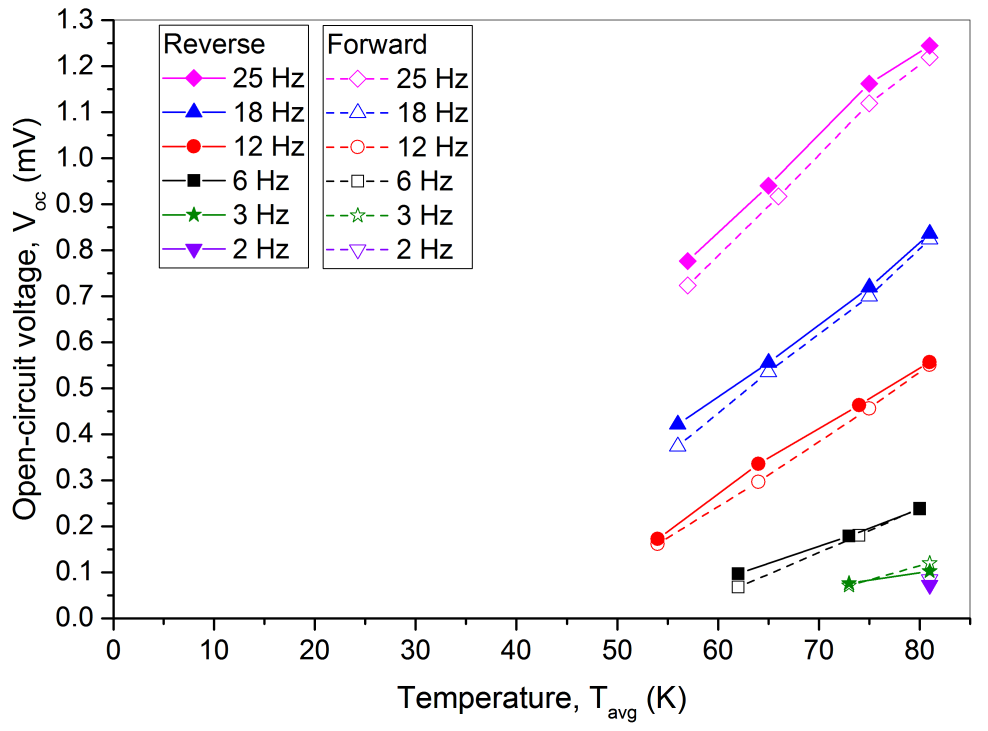

(a) Open-circuit voltage $\left(V_{o c}\right)$

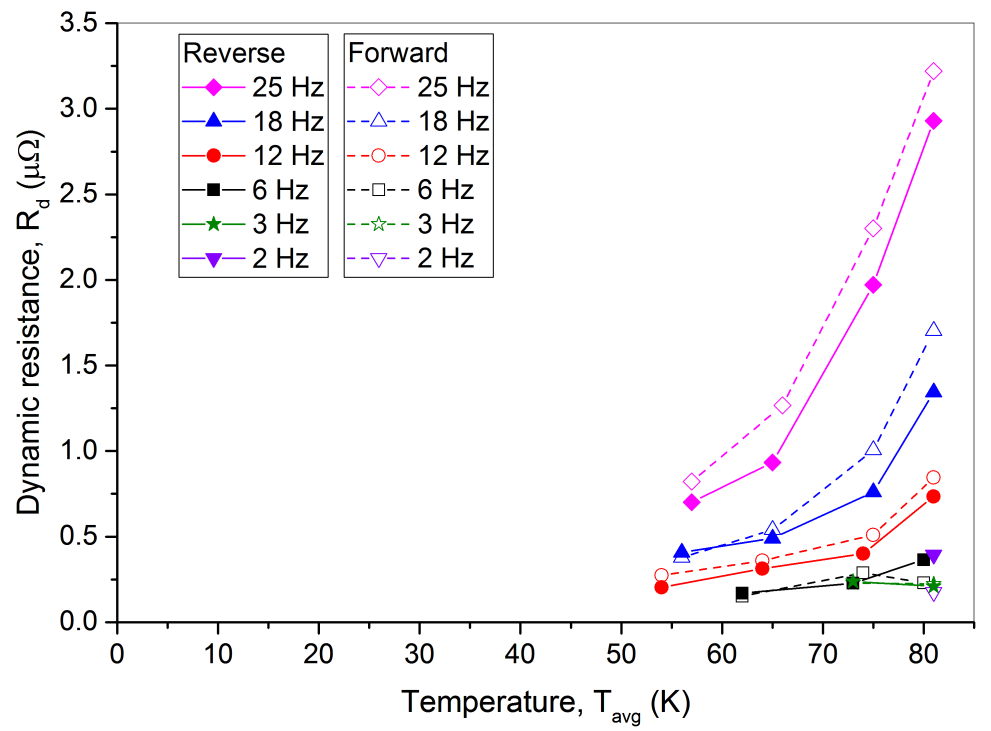

(b) Dynamic resistance $\left(R_{d}\right)$

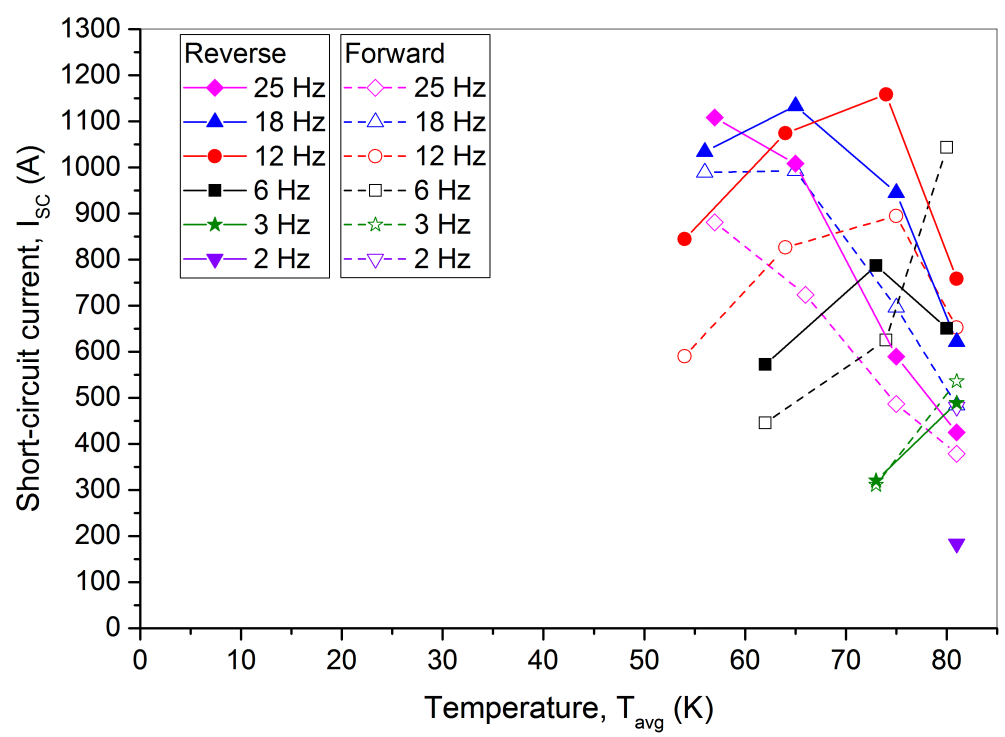

(c) Short-circuit current $\left(I_{s c}\right)$

Figure 6.9: FP performance as a function of the average stator temperature $T_{\text {avg }}$. The lines joining the data points are guides to the eye. 
In terms of the optimal performance of the FP, it desirable to have large $I_{s c}$ and large $V_{o c}$ (for high current pumping rate). It can be seen in figure $6.9 \mathrm{c}$, for the reverse direction, the maximum $I_{s c}$ at $74 \mathrm{~K}(12 \mathrm{~Hz}), 65 \mathrm{~K}(18 \mathrm{~Hz})$ and $57 \mathrm{~K}(25 \mathrm{~Hz})$ are comparable. However, figure $6.9 \mathrm{a}$ shows that among these temperatures the highest $V_{o c}$ occurs at $57 \mathrm{~K}(25 \mathrm{~Hz})$. This implies that, for these measurements, the FP performance is optimum when it is operating at $25 \mathrm{~Hz}$ at $57 \mathrm{~K}$.

\subsubsection{Frequency Normalised Plots}

Finally, in order to compare the effect of frequency on the data, the open-circuit voltage and the dynamic resistance were normalised by frequency. The plots are shown in figure 6.10. Both $V_{o c} / f$ and $R_{d} / f$ show a trend of increasing in value with frequency. This is very different from the results for Gen 1 (e.g. see figure 4.5) data where the frequency did not show a large effect on the normalised data. In fact, the data for Gen 1 systems was trending down with frequency due to the screening effect of the circulating current in the copper of the stator. The increase in value with frequency for the Gen 4 data might be due to heating effects in the stator. As the frequency increases, the heat generated in the stator increases. As a result the $\Delta T$ becomes larger and this increases the uncertainty in $T_{a v g}$. The non-linearity of the $I-V$ curves (figures 6.6 and 6.7) at high frequencies might also indicates that the stator is heated at higher frequency, leading to higher $R_{d} / f$ values.

Overall, the performance of the Gen 4 system in terms of both $V_{o c}$ and $I_{s c}$ is much higher than the Gen 1 and and Gen 2 systems. For example, the maximum $V_{o c} / f$ of the Gen 4 system is about 2.5 times and 7 times larger than the maximum $V_{o c} / f$ obtained from the Gen 2 and Gen 1 systems respectively. On the other hand, the maximum extrapolated $I_{s c}$ of the Gen 4 system is about 3.8 times and 18 times larger than the maximum $I_{s c}$ obtained from the Gen 2 and Gen 1 systems respectively. 


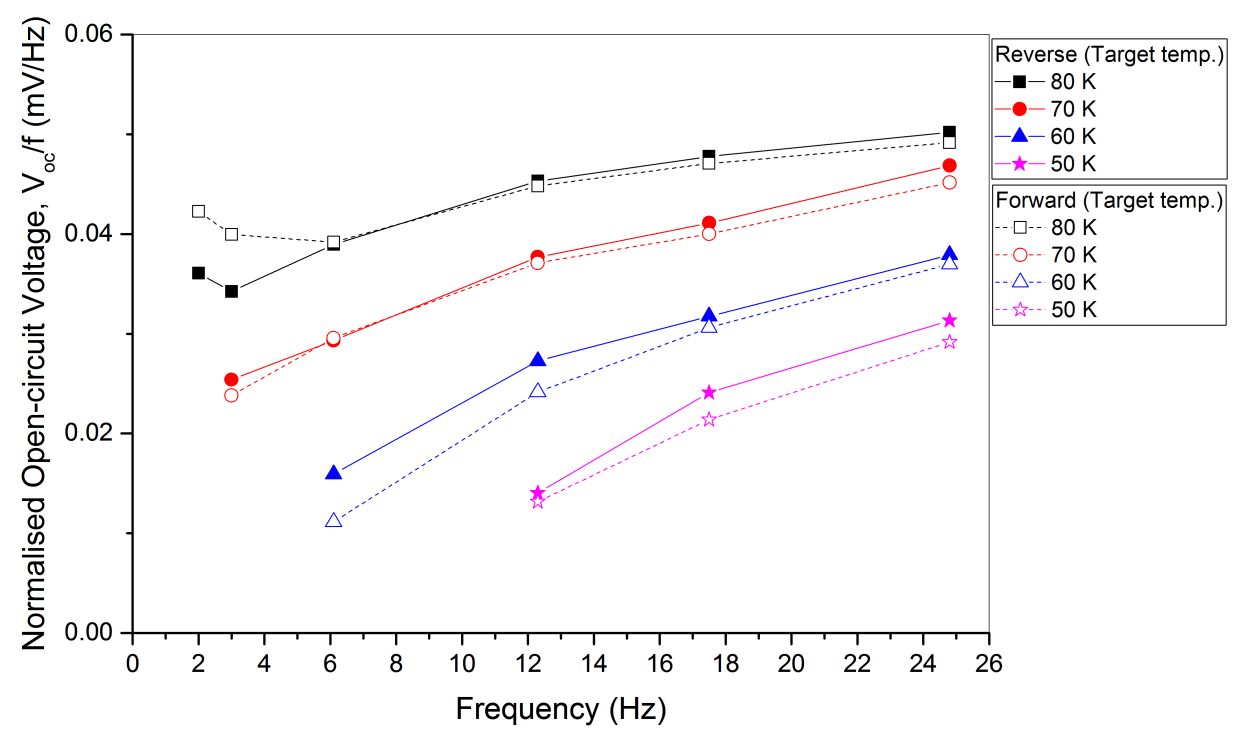

(a) Normalised open-circuit voltage $\left(V_{o c} / f\right)$

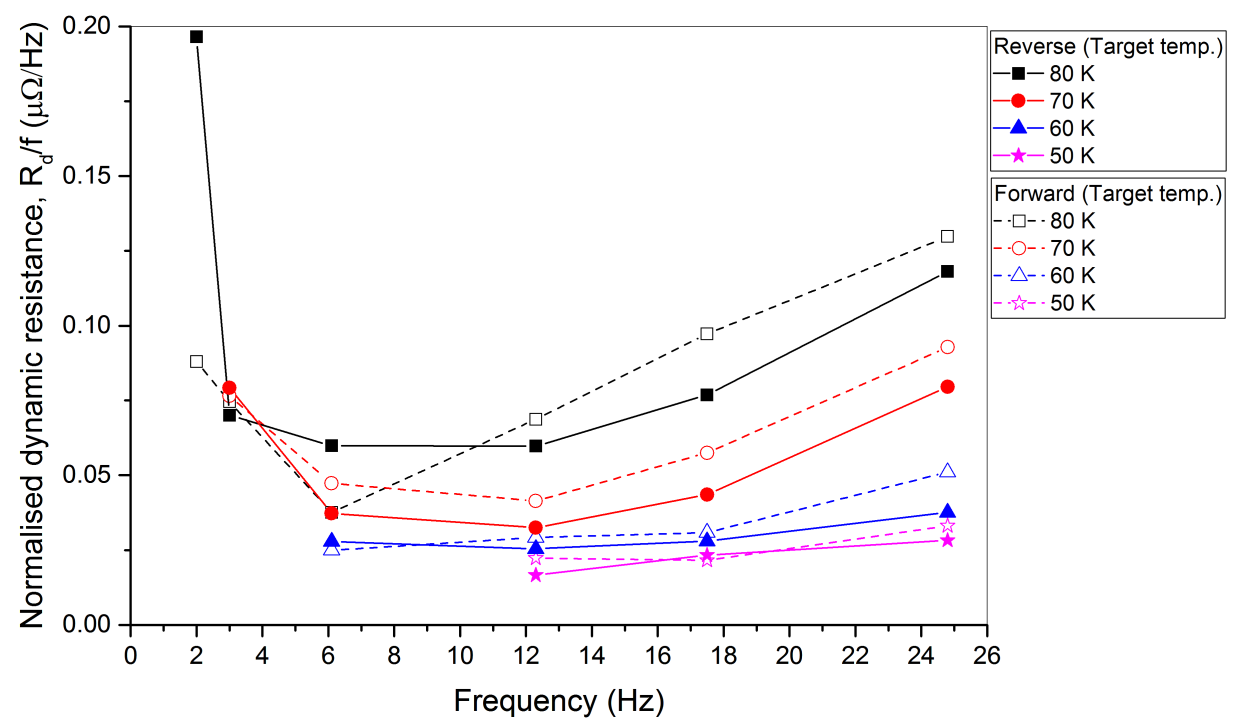

(b) Normalised dynamic resistance $\left(R_{d} / f\right)$

Figure 6.10: Frequency normalised plots. The lines joining the data points are guides to the eye.

\subsection{Summary}

Temperature stability was technically difficult to achieve in the experiment due to the large temperature difference between the two copper clamping plates. On top of that, the cooling power of the cryo-cooler is reduced at lower operating temperature. A method was employed in the experiment to achieve a stable stator temperature and the change in the temperature over time was smaller than $2 \mathrm{~K}$.

The analysis on the $\mathrm{AC}$ waveforms suggests that the mechanism that is responsible for the DC output voltage in the Gen 4 FP system is similar to the Gen 1 and Gen 2 FP systems. The non-zero average stator outputs of these HTS dynamo-type FPs are due to the change in the shapes of the $\mathrm{AC}$ waveforms measured in the superconducting state. In the case of the Gen 4 system the AC waveforms is rectified for all most the entire rotor cycle. Therefore 
the system is capable of producing a large DC output voltage.

Due to the geometry of the Gen 4 stator, the stator voltage is difficult to measure directly. Instead, the voltage across the current coil $V_{\text {coil }}$ was measured in the experiment. Since only the total contact resistance $R_{C T}$ in the FP circuit could be determined, the FP voltage was calculated from the sum of the voltage developed across $R_{C T}\left(V_{R_{C T}}\right)$ and $V_{\text {coil }}$.

The maximum current $I_{\text {sat }}$ in the FP circuit was much smaller than the extrapolated $I_{s c}$ of the FP. $I_{s a t}$ might have been limited by the $I_{c}$ of the current leads and flyleads and future works are required to investigate the leads and identify the source that is limiting the current.

Due to low $I_{\text {sat }}$ the uncertainty in the extrapolated $I_{s c}$ is very high. The possibility that $R_{d}$ is not constant at $I>I_{\text {sat }}$ cannot be ruled out.

The results for the forward and reverse rotor directions were very similar and this reflects the symmetry in the design of the stator. There was a high uncertainty in the stator average temperature $T_{\text {avg }}$ due to large $\Delta T$ and future work is required to reduce $\Delta V$. However, it is very clear that the performance of the Gen $4 \mathrm{FP}$, in terms of $V_{o c}$ and $I_{s c}$, is substantially higher compared with the Gen 1 and Gen 2 systems.

Finally, the frequency-normalised plots $V_{o c} / f$ and $R_{d} / f$ appeared to increase with frequency and this could be due to high uncertainty in $T_{a v g}$. 


\section{Chapter 7}

\section{Conclusions}

The Gen 1, Gen 2 and Gen 4 flux pump systems studied in this project all have different designs and cooling arrangements. However, they all employed high temperature superconductors and are classified as mechanically rotating flux pumps. All three systems have a rotor, permanent magnets and stator and produce a DC voltage through electromagnetic induction. Their DC operation can be described using simple DC equivalent circuits. This allows the performance of these systems to be characterised by three DC parameters: opencircuit voltage, dynamic resistance and short-circuit current.

\subsection{Gen 1 System}

In the experiments the effects of the magnet geometry on the FP performance were investigated. The results showed that the dynamic resistance of the stator depended on the strength of the magnetic field and the interaction length between the field and the stator. The frequency-normalised dynamic resistance decreased with the operating frequency of the FP due to the eddy-current shielding effect. The orientation of the magnet did not have a strong effect on the dynamic resistance.

The system was capable of producing a DC output voltage because the AC voltage waveform measured across the stator in the superconducting state was partially rectified. The open-circuit voltage of the FP increased with magnetic field strength and the magnet length, measured along the long-length axis of the stator. The open-circuit voltage also increased with the FP operating frequency.

The short-circuit current of the FP is equal to the open-circuit voltage divided by the dynamic resistance. The changes in the open-circuit voltage with respect to frequency cancelled out the changes in the dynamic resistance. As a result, the output current was stable across the frequency range studied. In general, magnet geometries which led to low dynamic resistance exhibited higher output currents. 


\subsection{Gen 2 System}

Three types of stators were used in the experiments to investigate the effect of the FP operating frequency on the performance of the system. All stators were made from $46 \mathrm{~mm}$ wide Ag-coated HTS tape. The first stator had a full $46 \mathrm{~mm}$ width. The second stator was slit to form two parallel stators with equal width. The third stator was slit to form three parallel stators with equal width.

Each stator could produce a non-zero DC output voltage and this was attributed to the partial rectification of the $\mathrm{AC}$ waveform measured across the stator in superconducting state.

Three FP operation frequency regimes were observed in the experiments: low, mid and high. At low frequency, the FPs open-circuit voltage and dynamic resistance increased linearly with frequency. In the mid frequency regime, the rate of change of the dynamic resistance with respect to frequency was higher compared with the low frequency regime. This is attributed to the onset of local heating, especially in the slit stators where the width of the individual stators was smaller. In some cases, two thermally stable operating modes were observed and each mode had a different set of FP performance parameters. At high frequency, the dynamic resistance increased dramatically and the open-circuit voltage decreased. As a result, the output current approached zero.

The AC voltage waveforms measured across the stators served as good tools in analysing the FP operation mode because the shape of the waveforms was different in each operating frequency regime.

Overall, the results showed that the performance of the Gen 2 FP was frequency dependent and that the slit stators were thermally less stable compared with the full stator.

\subsection{Gen 4 System}

The stator of the Gen 4 system generated heat when the FP was in operation. This resulted in large temperature difference between the copper clamping plates that held the stator in place. For this reason there was a high uncertainty in the average stator temperature.

As in the Gen 1 and Gen 2 system, the Gen 4 system was capable of producing a DC output voltage because the $\mathrm{AC}$ voltage waveform in the superconducting state was partially rectified. The rectification in the Gen 4 system occurred for almost the entire rotor cycle. As a result, it produced larger open-circuit voltages and was far more effective when compared to the Gen 1 and Gen 2 systems.

The Gen 4 system produced very low $I_{\text {sat }}$, and this is believed due to damaged current leads. For this reason, the range of data in the $I-V$ curve for the line fit was very small and the extrapolated short-circuit currents of the FP had very high uncertainty. The possibility that $R_{d}$ is not constant at current greater that $I_{\text {sat }}$ cannot be ruled out. 


\subsection{Applications}

The large flux gap of the Gen 2 system allows for a thick insulating material to be used in the cryostat wall. The moving parts are located external to the cryogenics environment and this minimises the heat loss. The Gen 2 FP has been previously used to characterise a prototype HTS-PM brushless exciter, which was implemented in a $10 \mathrm{~kW}$ HTS generator $[4]$.

The overall results of the Gen 4 system seemed to indicate that the FP is capable of producing currents in the $\mathrm{kA}$ range. This capability is useful in applications where large output current is required. An example is to supply a constant current to a HTS magnet coil in a NMR system. However, future work is required on the Gen 4 system before it can be used in commercial applications. One important area of study is to investigate the components that were limiting the $I_{\text {sat }}$ in the experiments. The limiting factor was believed to be due to the damaged current leads. However, other possibilities, such as the HTS current coil and the stator, are to be investigated as well. In this project, only the $12 \mathrm{~mm}$-size magnet was installed in the rotor. In future, other magnet sizes (3 $\mathrm{mm}$ and $6 \mathrm{~mm}$ ) and orientations can be used to characterise the FP performance. The objective is to demonstrate that it is feasible to build a kA-class HTS FP based on the Gen 4 system design. 


\section{Bibliography}

[1] AREPOC. High Linearity Hall Probes for Room and Cryogenic Temperatures, 2008.

[2] Atherton, D. L. Superconducting de generators and motors. IEEE Spectrum 1, 12 (1964), 67-71.

[3] Badcock, R. A., Phang, S., Pantoja, A. E., Jiang, Z., Storey, J. G., Sung, H.-J., PARK, M., AND Bumby, C. W. Impact of magnet geometry on output of a dynamo-type HTS flux pump. IEEE Transactions on Applied Superconductivity 27, 4 (2016), 5200905.

[4] Bumby, C. W., Badcock, R. A., Sung, H.-J., Kim, K.-M., Jiang, Z., Pantoja, A. E., Bernardo, P., Park, M., and Buckley, R. G. B. R. G. Development of a brushless HTS exciter for a $10 \mathrm{~kW}$ HTS synchronous generator. Superconductor Science and Technology 29, 2 (2016), 024008 (11pp).

[5] Bumby, C. W., Jiang, Z., Storey, J. G., Pantoja, A. E., , And Badcock, R. A. Anomalous Open-circuit Voltage From a High- $T_{c}$ Superconducting Dynamo. Applied Physics Letters 108, 12 (2013), 122601.

[6] Bumby, C. W., Pantoja, A. E., Sung, H. J., Jiang, Z., Kulkarni, R., And BADCOCK, R. A. Through-Wall Excitation of a Magnet Coil by an External-Rotor HTS Flux Pump. IEEE Transactions on Applied Superconductivity 26, 4 (2016), 0500505.

[7] Bumby, C. W., Phang, S., Pantoja, A. E., Jiang, Z., Storey, J. G., Sung, H.-J., PARK, M., AND BADCOCK, R. A. Frequency dependent behaviour of a dynamotype HTS Flux Pump. IEEE Transactions on Applied Superconductivity 27, 4 (2016), 5200705 .

[8] Cryomech. AL63 Cryorefrigerator Capacity Curve.

[9] Cryomech. Cryogenic refrigerator - installation, operation and routine maintenance manual, January 2007.

[10] Cyrot, M., and Pavuna, D. Introduction To Superconductivity and High-T Material. World Scientific, 1992.

[11] Ekin, J. W. Experimental Techniques for Low-Temperature Measurements. Oxford Universtity Press, 2007. 
[12] Geng, J., Matsuda, K., Fu, L., Fagnard, J.-F., Zhang, H., Zhang, X., Shen1, B., Dong, Q., Baghdadi, M., And Coombs, T. A. Origin of dc voltage in type II superconducting flux pumps: field, field rate of change, and current density dependence of resistivity. Journal of Physics 49, 11 (2016), 11LT01 (5pp).

[13] Giaever, I. A DC Transformer. IEEE Spectrum 3, 9 (Sept. 1966), 117-122.

[14] Hoffmann, C., Pooke, D., And Caplin, A. D. Flux Pump for HTS Magnets. IEEE Transactions on Applied Superconductivity 21, 3 (2011), 1628 - 1631.

[15] Hoffmann, C., Walsh, R., Karrer-Mueller, E., And Pooke, D. Design parameters for an HTS flux pump. Physics Procedia 36 (2012), 1324 - 1329.

[16] Horowitz, P., And Hill, W. The Art of Electronics. Cambridge University Press, 1989.

[17] Jiang, Z., Bumby, C. W., Badcock, R. A., Sung, H.-J., Long, N. J., And Amemiya, N. Impact of flux gap upon dynamic resistance of a rotating HTS flux pump. Superconductor Science and Technology 28 (2015), 115008 (9pp).

[18] Jiang, Z., Hamilton, K., Amemiya, N., Badcock, R. A., , And Bumby, C. W. Dynamic resistance of a high-Tc superconducting flux pump. Applied Physics Letters 105 (2014), 112601.

[19] Jiang, Z., Toyomoto, R., Amemiya, N., Zhang, X., And Bumby, C. W. Dynamic resistance of a high- $T_{c}$ coated conductor wire in a perpendicular magnetic field at 77 K. Superconductor Science and Technology 30, 03 (2017), 03LT01 (7pp).

[20] LakeShore Cryotronics. Sensor Packaging and Installation. Lake Shore Cryotronics.

[21] LakeShore Cryotronics. DT670 Silicon Diodes Specifications. Lake Shore Cryotronics, May 2016.

[22] Linear Technology. Precision Operational Amplifier, b ed. Linear Technology.

[23] Mawardi, O. K., Gattozzi, A., And Chung, H. Operational characteristics of a flux pump. IEEE Transaction on magnetics 15, 1 (Jan. 1979), 828 - 831.

[24] Mawardi, O. K., Muelder, S. A., And Michelotti, R. A. Brushless superconducting alternators. IEEE Transaction on Magnetics 13, 1 (Jan. 1977), 780 - 783.

[25] Maxim Intergrated. MAX6225/MAX6241/MAX6250, 5 ed. Maxim Intergrated, 2015.

[26] National Instruments. NI 9263 4 Ch Module. National Instruments, 112014.

[27] National Instruments. NI 9205 Datasheet. National Instruments, Oct. 2015. 
[28] National Instruments. How To Measure Voltage, May 2016.

[29] On Semicondcutor. MJH11022 (NPN) Complementary Darlington Silicon Power Transistors, 10 ed. On Smiconductor, August 2016.

[30] Oomen, M. P., Rieger, J., Leghissa, M., ten Haken, B., And ten Kate, H. H. J. Dynamic resistance in a slab-like superconductor with $J_{c}(B)$ dependence. Superconductor Science and Technology 12 (1999), 382 - 387.

[31] Pantoja, A. E., Jiang, Z., Badcock, R. A., , And Bumby, C. W. Impact of Stator Wire Width on Output of a Dynamo-Type HTS Flux Pump. IEEE Transactions on Applied Superconductivity 26, 8 (2016), 4805208.

[32] Pfeiffer-Vacuum. HiCube Classic. Pfeiffer Vacuum.

[33] Rhyner, J. Magnetic properties and AC-losses of superconductors with power law current-voltage characteristics. Physica C 212 (1993), 292-300.

[34] Saint-Gobain. Sapphire Products- Properties and Benefits, 2014.

[35] SuperPower Inc. SuperPower 2G HTS Wire Specifications. SuperPower Inc., 2014.

[36] Texas Instruments. LM101, LM103 AN-32 FET Circuit Applications. Texas Instruments, 2011.

[37] Texas Instruments. LM334 Terminal Adjustable Current Sources. Texas Instruments, May 2013.

[38] Texas Instruments. LMx24-N, LM2902-N Low-Power, Quad-Operational Amplifiers. Texas Instruments, 012015.

[39] VAn DE Klundert, L., AND TEn KATE, H. Fully superconducting rectifiers and fluxpumps. Cryogenics 21, 4 (1981), 195-206.

[40] Walsh, R. M., Slade, R., Pooke, D., , and Hoffmann, C. Characterization of Current Stability in an HTS NMR System Energized by an HTS Flux Pump. IEEE Transactions on Applied superconductivity 24, 3 (2014), 1-5. 


\section{Appendix A}

\section{0-watt Constant-current Heater Power Supply}

\section{A.1 Heater Circuit Design}

The design objective was to build a power supply that can deliver a constant current to heating elements installed on the copper clamping plates of the FP. The power supply must have the facility to allow for the implementation of a feedback loop and a controller (e.g. PID controller) so that the amount of power delivered to the heating elements can be controlled (see figure 5.11 for the schematic). During the process of cooling and heating, the resistance of the the heating elements is expected to change. Therefore, the power supply has to be able to maintain a constant current level.

\section{A.1.1 Voltage-controlled Precision Current Sink}

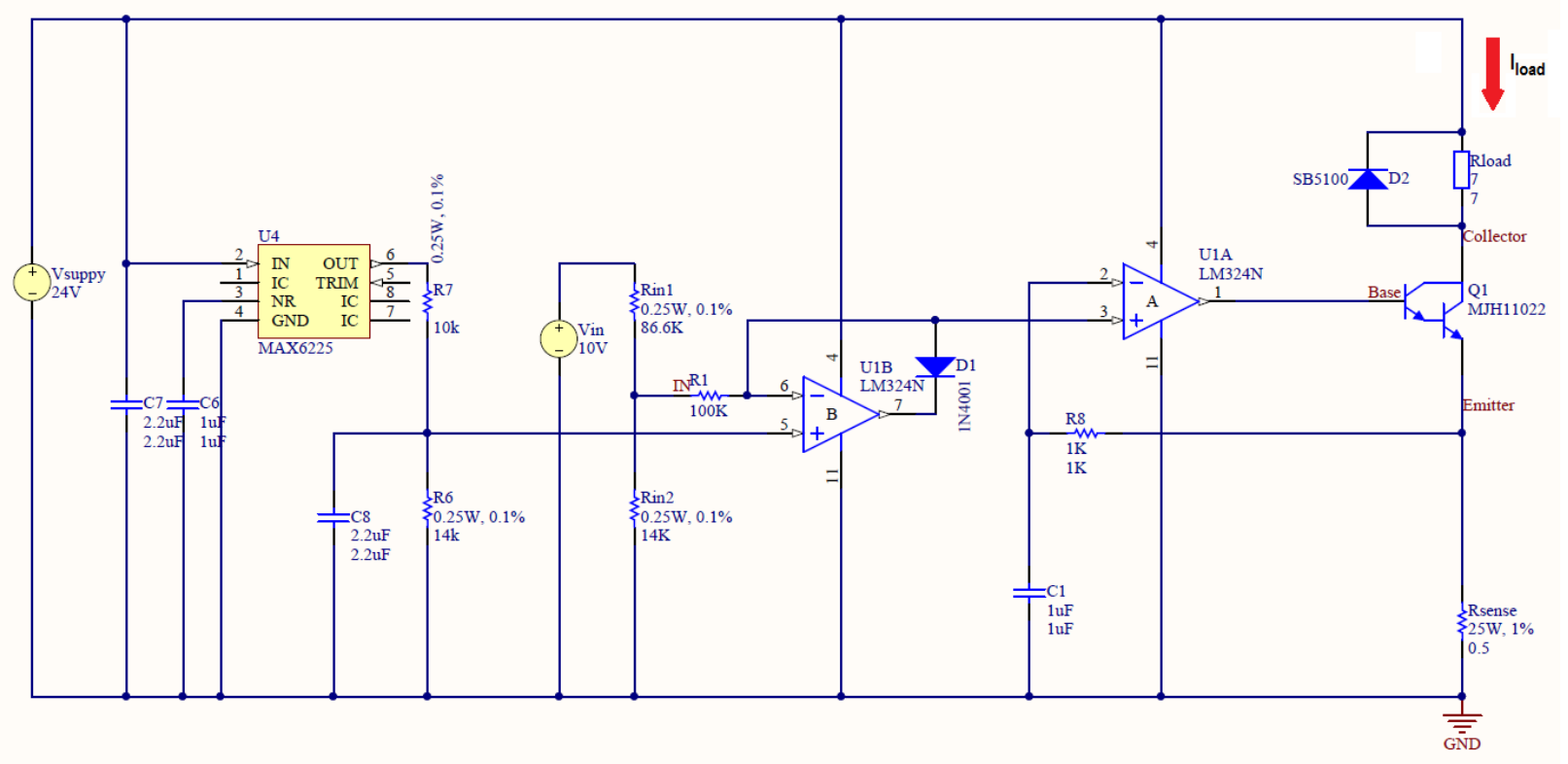

Figure A.1: A 50-watt voltage-controlled precision current sink. 
A common technique in supplying a constant current is to use an op-amp and configure it as a non-inverting amplifier with a negative feedback $[22,36,16]$. This is known as a precision current sink and the circuit is shown in figure A.1. Here, the simplified version of the final design is shown so that the major circuit components used in the circuit can be discussed.

The advantage of the circuit is that the load current $I_{\text {Load }}$ is a function of the control voltage $V_{i n}$ and the feedback resistor $R_{\text {sense }}$. This is given in equation A.1 below:

$$
I_{\text {Load }}=\frac{0.14 \times V_{\text {in }}}{R_{\text {sense }}}
$$

This simple relationship implies that the load current does not depend on the load resistance $R_{\text {load }}$. It is also insensitive to temperature variations, provided that $R_{\text {sense }}$ is given an adequate cooling. This can be achieved easily by mounting it to a heat sink.

\section{A.1.2 The Power Transistor}

The MJH11022 Darlington pair power transistor is chosen because it allows for the load current to be controlled linearly. In addition, it also has high DC current gain (400 @ 10 A), high operating junction temperature $\left(150^{\circ} \mathrm{C}\right)$, low junction-to-case thermal resistance $(0.83$ $\left.{ }^{\circ} \mathrm{C} / \mathrm{W}\right)$, low collector-emitter saturation voltage (1.2 V @ $\left.5.0 \mathrm{~A}\right)$ and high power handling (max. $150 \mathrm{~W}$ at case temperature $25^{\circ} \mathrm{C}$ ) [29].

\section{A.1.3 NI-9263 Maximum Control Voltage and Sensing Resistor Value}

The input voltage $V_{\text {in }}$ is supplied by the National Instrument NI-9263 [26] analogue output module. It has a 16-bit output resolution and the maximum output voltage of $10 \mathrm{~V}$ is used for the highest control resolution.

The resistors $R_{i n 1}$ and $R_{i n 2}$ form a voltage divider with an output-to-input ratio of 0.14 (this is where the 0.14 factor in equation A.1 came from). At maximum input $10 \mathrm{~V}$ the output of the divider is $1.4 \mathrm{~V}$. This is supplied to to pin 3 of the op-amp U1A via the voltage clamp circuit (U1B, R1, R2, R3 and D1). The purpose of the voltage clamp is to ensure that the voltage at pin 3 of the op-amp does not rise higher than $1.4 \mathrm{~V}$. The reference voltage for the voltage clamp is supplied by U4. It is a low noise precision voltage reference Max6225 [25], manufactured by Maxim Integrated.

The op-amp is LM324 [38], manufactured by Texas Instrument. It was chosen because it can operate from a single supply. It does not draw any current at the inputs due to high input impedance. Therefore, the voltage at pin 2 of the op-amp must be equal to the voltage developed across $R_{\text {sense }}$. In order to bring both pins 2 and 3 to the same voltage level U1A drives Q2, which in turn changes the load current until $1.4 \mathrm{~V}$ is developed across $R_{\text {sense }}$. The high current gain of the Darlington power transistor implies that the the current in $R_{\text {sense }}$ is always equal to the load current $\left(I_{E} \approx I_{C}\right)$. The maximum power required for the load is $50 \mathrm{~W}$. At this power level, the current in the $7 \Omega$ load is $2.7 \mathrm{~A}$. Apply $I_{\text {Load }}=2.7 \mathrm{~A}$ and 
$V_{i n}=10 \mathrm{~V}$ to equation A.1 and this results in $R_{\text {sense }}=0.5 \Omega$. At the maximum current the power dissipated in $R_{\text {sense }}$ is only $4 \mathrm{~W}$, which can be cooled by a heat sink.

\section{A.1.4 Other Considerations}

The RC network of $1 \mathrm{k} \Omega$ and $1 \mu \mathrm{F}$ has a time constant of about $1 \mathrm{~ms}$ (cut-off frequency of about $160 \mathrm{~Hz}$ ). This is sufficient to filter out any high-frequency transient signal. $R_{i n 2}$ of the voltage divider also acts as a pull-down resistor to prevent the op-amp input from floating when $V_{i n}$ is disconnected. If a coil is used as the load, a substantial amount of energy could be stored in it while the transistor is turned off. Therefore, a free-wheeling diode is installed across the load to allow for the coil energy to dissipate.

\section{A.2 Heatsink and Transistor Junction Temperature}

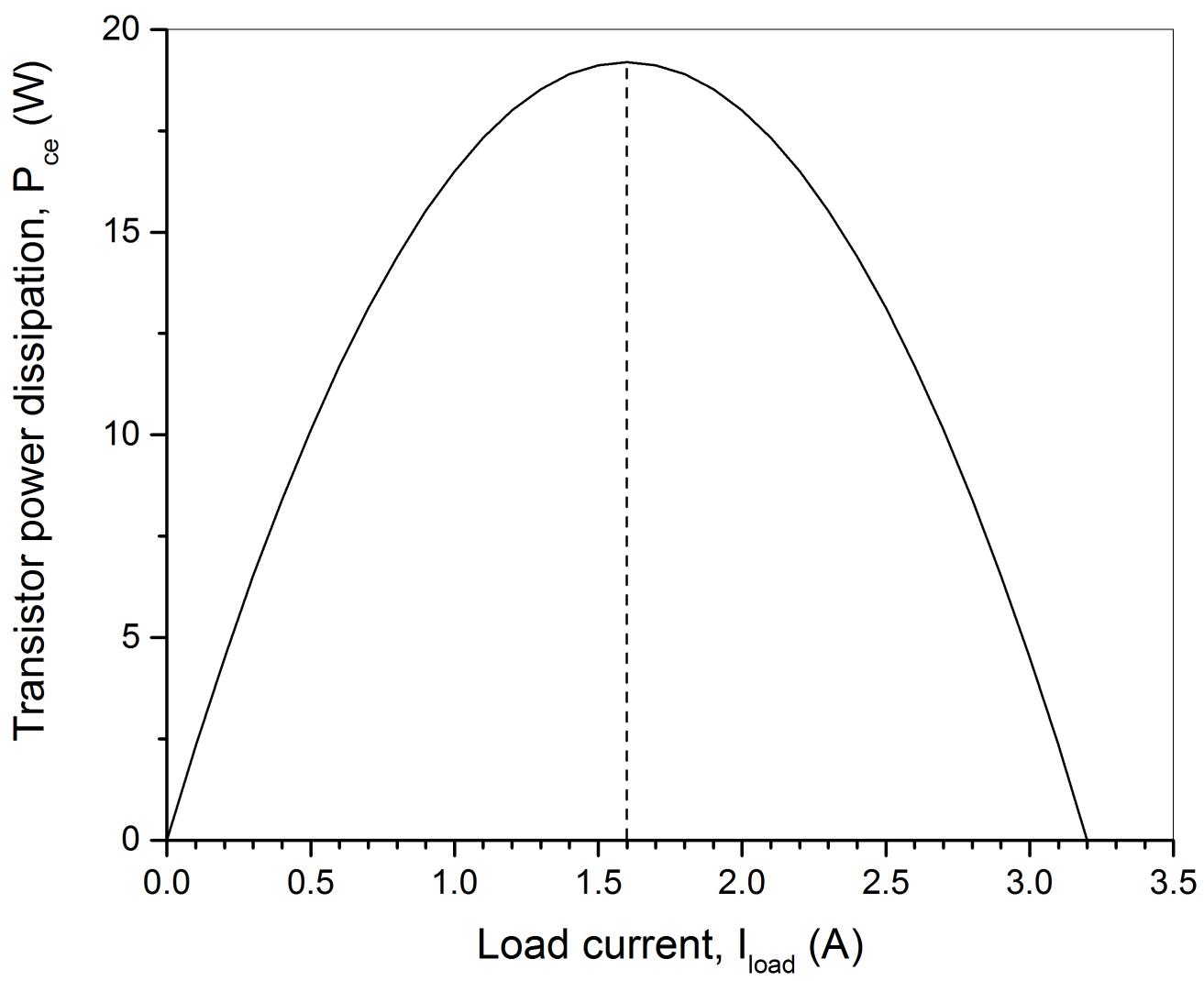

Figure A.2: Power transistor power dissipation $P_{c e}$ as a function of load current $I_{\text {load }}$

Figure A.2 shows the power dissipation $P_{c e}$ of the power transistor as a function of load current $I_{\text {load }}$. The maximum transistor power dissipation is $19.2 \mathrm{~W}$, which occurs at $I_{\text {load }}=$ $1.6 \mathrm{~A}$. 


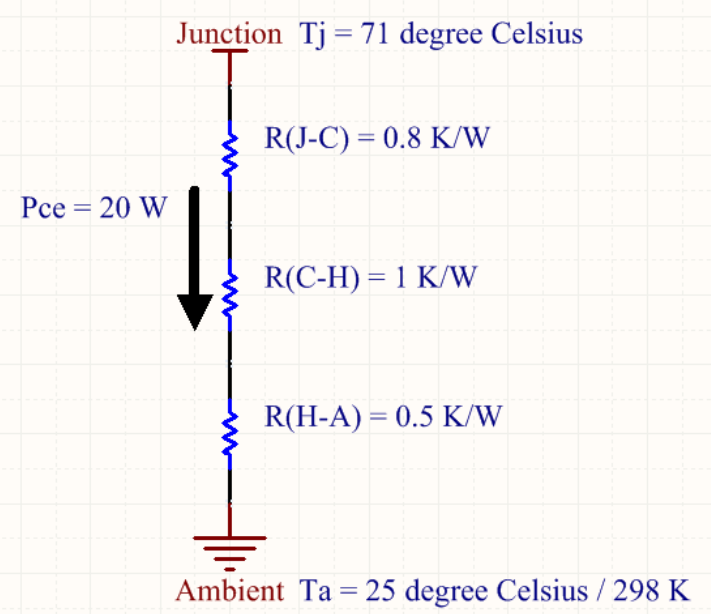

Figure A.3: Thermal Modelling. $P_{c e}=$ transistor power dissipation, $T=$ temperature, $j=$ junction, $C=$ transitor case, $H=$ heatsink, $a=$ ambient, $R=$ thermal resistance

Figure A.3 shows the thermal model of the heat transfer from the transistor junction to ambient. The transistor junction-to-case thermal resistance is $0.8 \mathrm{~K} / \mathrm{W}[29]$ and the case-to-heatsink resistance (including the insulating washer and thermal paste) is estimated to be $1 \mathrm{~K} / \mathrm{W}$. A heatsink of $0.5 \mathrm{~K} / \mathrm{W}$ was used for the project. The maximum power dissipation of the transistor is assumed to be $20 \mathrm{~W}$. The thermal circuit parameters and analysis are analogous to electrical circuit analysis. In this case power is equal to current, temperature is equal to voltage and heat resistance is equal to electrical resistance. The junction temperature is described by the equation:

$$
T_{J}=P_{c e}\left(R_{J C}+R_{C H}+R_{H A}\right)+T_{a}
$$

The result is $T_{J}=71^{\circ} \mathrm{C}$, which is less than half of the maximum operating junction temperature $\left(T_{J_{M A X}}=150{ }^{\circ} \mathrm{C}\right)$ that the transistor tolerates for safe operation. 


\section{A.3 Prototype Test and Final Design}

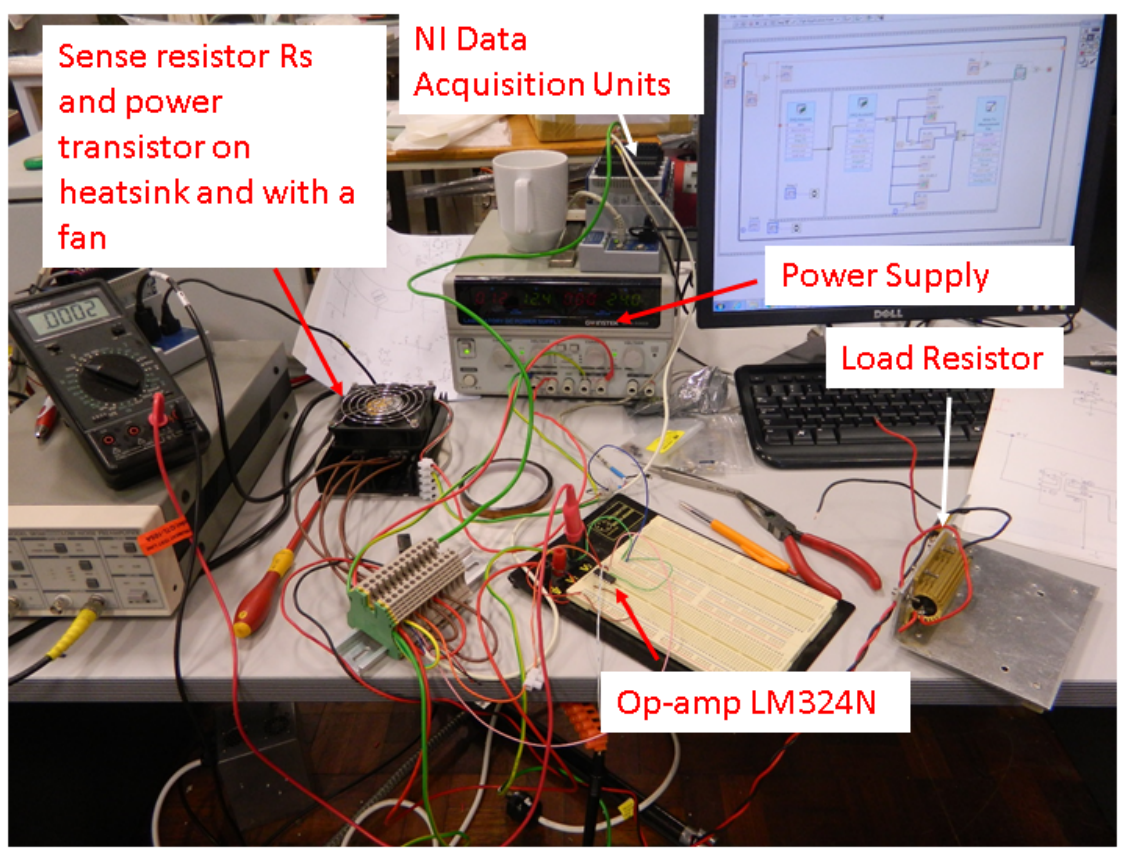

Figure A.4: Testing the heater power supply prototype.

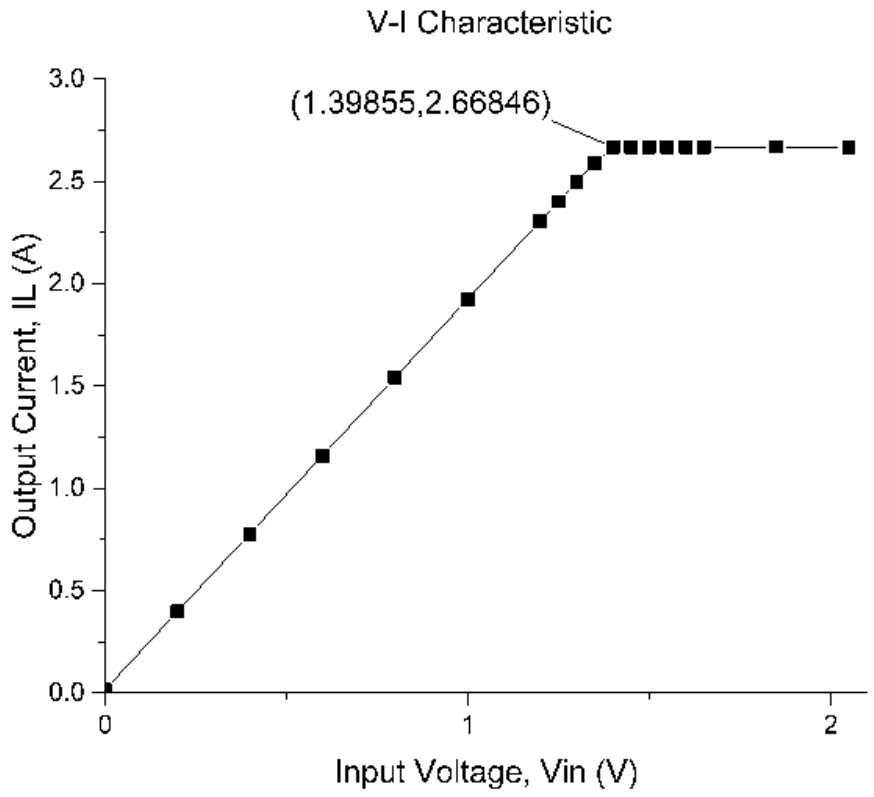

Figure A.5: Heater power supply test result.

Figure A.4 shows the testing of the prototype of the circuit and figure A.5 shows the V-I characteristic obtained using a $7 \Omega$ test load. The result shows that the V-I characteristic is linear up to $2.7 \mathrm{~A}$. It stays constant at this level and the power delivered to the load is 51 W. 


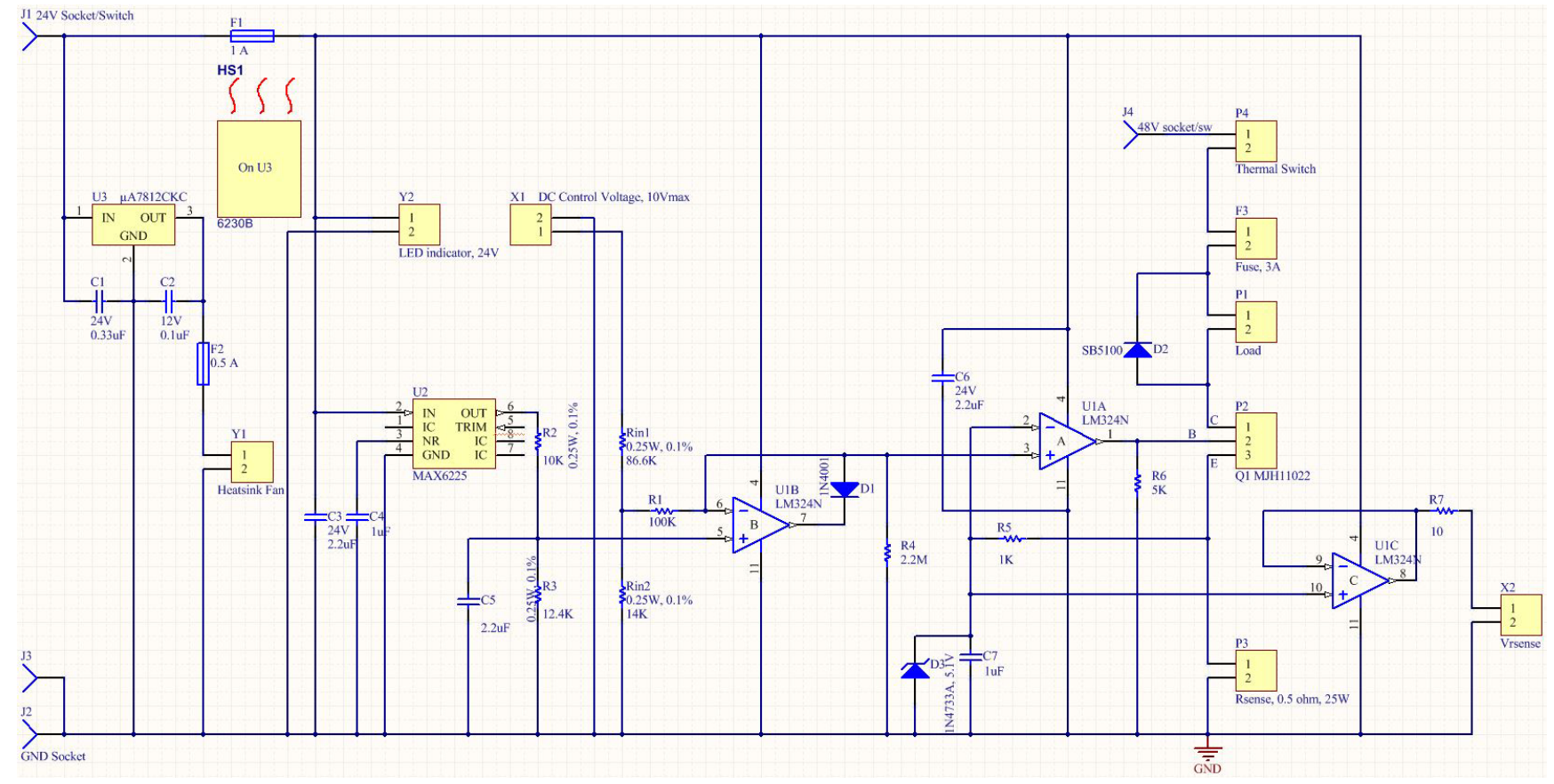

Figure A.6: The final design of the 50-watt voltage-controlled heater power supply.

The final circuit design is shown in figure A.6. It is designed for PCB construction, therefore, the externally mounted components are replaced with a connector. Example of the external components are the power transistor, fuses and the current sense resistor. A $12-\mathrm{V}$ voltage regulator UA7812 is included for the heatsink fan power. 


\section{Appendix B}

\section{0- $\mu$ A Constant Current Source for DT-670 Diode Sensors}

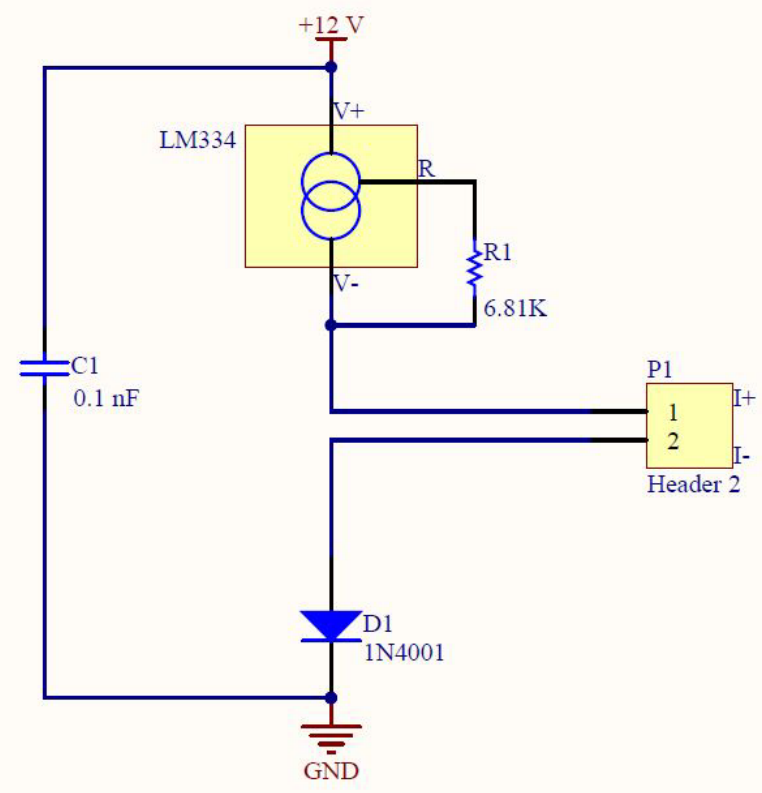

Figure B.1: $10 \mu \mathrm{A}$ constant current excitation circuit for DT-670 diode sensors using the LM334 [37] adjustable current source. The output P1 supplies the excitation to the diode. D1 is the diode for reverse voltage terminal protection. 